Old Dominion University

ODU Digital Commons

Physics Theses \& Dissertations

Physics

Spring 2017

\title{
Nonlinear Dynamics of Vortices in Different Types of Grain Boundaries
}

Ahmad K. Sheikhzada

Old Dominion University

Follow this and additional works at: https://digitalcommons.odu.edu/physics_etds

Part of the Condensed Matter Physics Commons

\section{Recommended Citation}

Sheikhzada, Ahmad K.. "Nonlinear Dynamics of Vortices in Different Types of Grain Boundaries" (2017). Doctor of Philosophy (PhD), Dissertation, Physics, Old Dominion University, DOI: 10.25777/mzgg-t868 https://digitalcommons.odu.edu/physics_etds/8

This Dissertation is brought to you for free and open access by the Physics at ODU Digital Commons. It has been accepted for inclusion in Physics Theses \& Dissertations by an authorized administrator of ODU Digital Commons. For more information, please contact digitalcommons@odu.edu. 


\title{
NONLINEAR DYNAMICS OF VORTICES IN DIFFERENT TYPES OF GRAIN BOUNDARIES
}

\author{
by
}

\author{
Ahmad K. Sheikhzada \\ B.Sc. May 2008, Amirkabir University of Technology, Iran \\ M.Sc. May 2012, Old Dominion University
}

A Dissertation Submitted to the Faculty of

Old Dominion University in Partial Fulfillment of the

Requirements for the Degree of

DOCTOR OF PHILOSOPHY

PHYSICS

OLD DOMINION UNIVERSITY

May 2017

Approved by:

Alex Gurevich (Director)

Jean Delayen (Member)

Alex Godunov (Member)

Steven Pascal (Member)

Todd Satogata (Member) 


\title{
ABSTRACT \\ NONLINEAR DYNAMICS OF VORTICES IN DIFFERENT TYPES OF GRAIN BOUNDARIES
}

\author{
Ahmad K. Sheikhzada \\ Old Dominion University, 2017 \\ Director: Dr. Alex Gurevich
}

As a major component of linear particle accelerators, superconducting radio-frequency (SRF) resonator cavities are required to operate with lowest energy dissipation and highest accelerating gradient. SRF cavities are made of polycrystalline materials in which grain boundaries can limit maximum RF currents and produce additional power dissipation sources due to local penetration of Josephson vortices. The essential physics of vortex penetration and mechanisms of dissipation of vortices driven by strong RF currents along networks of grain boundaries and their contribution to the residual surface resistance have not been well understood. To evaluate how GBs can limit the performance of SRF materials, particularly $\mathrm{Nb}$ and $\mathrm{Nb}_{3} \mathrm{Sn}$, we performed extensive numerical simulations of nonlinear dynamics of Josephson vortices in grain boundaries under strong dc and RF fields. The RF power due to penetration of vortices both in weakly-coupled and strongly-coupled grain boundaries was calculated as functions of the RF field and frequency. The result of this calculation manifested a quadratic dependence of power to field amplitude at strong RF currents, an illustration of resistive behavior of grain boundaries. Our calculations also showed that the surface resistance is a complicated function of field controlled by penetration and annihilation of vortices and antivortices in strong RF fields which ultimately saturates to normal resistivity of grain boundary. We found that Cherenkov radiation of rapidly moving vortices in grain boundaries can produce a new instability causing generation of expanding vortexantivortex pair which ultimately drives the entire GB in a resistive state. This effect is more pronounced in polycrystalline thin film and multilayer coating structures in which it can cause significant increase in power dissipation and results in hysteresis effects in I-V characteristics, particularly at low temperatures. 
Copyright, 2017, by Ahmad K. Sheikhzada, All Rights Reserved. 


\section{ACKNOWLEDGMENTS}

I would like to thank first and foremost my research adviser Dr. Alex Gurevich who gave me the opportunity to work with him, who shared with me generously his vast knowledge and guided me through this journey with enormous patience and friendship. Alex taught me the basics, was always available for my questions and supported me in every aspect of research, whether it was a publication, a presentation or application.

My special thank goes to Dr. Gail Dodge who was the chair of the department when I joined, who suggested me to work with Alex and supported me during hard times. I also sincerely thank Dr. Charles Sukenik, our current chair of the department for great management and successful administration.

I am greatly indebted to Dr. Jean Delayen, director of center for accelerator science where I spent most of my time. Jean realizes a true professor, mentor and physicist whose courses were sources of nourishment to me. Beside that, his questions and critique were always insightful during my talks.

Special thanks to Dr. Alex Godunov, Dr. Todd Satogata and Dr. Steven Pascal who accepted to give me company along this journey as committee members whose ideas and critique were great sources of improvement to my work.

I would like to thank Dr. Gianluigi Ciovati for being my adviser at Jefferson lab, helpful discussions and recommendations.

I am particularly thankful to Dr. Lephsha Vuskovic, our graduate program director whose continuous advice made this goal more feasible to achieve.

I am grateful to Mrs. Lisa Okun for not only being the first source of help, but a great noble lady whose personality, ever-present smile and friendly conversations are treasures for the physics department.

Many thanks go to all my professors in the department whose passion for science, intelligence and hard work made the courses fruitful and joyous. I am sincerely grateful and indebted to all staff at both Physics Department and Office of VISA for international affairs whom without their hard work, enormous help and useful consultations this would have never been possible.

I am deeply indebted to my uncle who has been a great friend and a strong support from

day first I came to USA. I specially thank his wife, his great children and wish them all success and happy life.

I would like to express my special debt of gratitude to my wife whose presence and 
patience supported this work in many ways. I am extremely grateful for having wonderful brothers and sisters whose ever-present love and support has always been a great asset for me and I wish them all success and happiness.

Finally, if there is any success in my life, I owe it all to two angels in my life without their presence I have none in this world. My father who always dreamed big for us, supported us in every aspect of life, advised us step by step and still continues to sacrifice what he has got in order to give us what we need. My mother who is a true angel, the statue of love and the mountain of mercy. She taught me love and kindness, patience and perseverance, sincerity and honesty.

Last but not least I would like to express my sense of gratitude to all my teachers and professors at Shahid Ghaffari school, Shahed Ali Akbar high school and Physics Department of Amirkabir University. I wish all of you long, happy and successful life. 


\section{TABLE OF CONTENTS}

\section{Page}

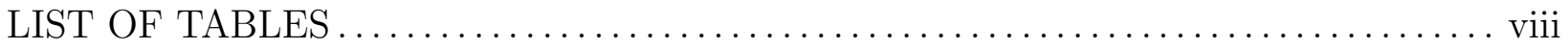

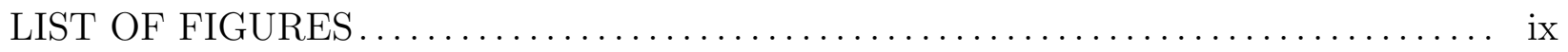

Chapter

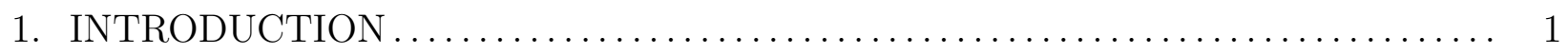

1.1 GRAIN BOUNDARIES IN SRF MATERIALS $\ldots \ldots \ldots \ldots \ldots \ldots \ldots \ldots \ldots \ldots$

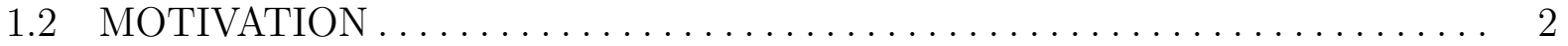

1.3 ORGANIZATION OF DISSERTATION $\ldots \ldots \ldots \ldots \ldots \ldots \ldots \ldots \ldots \ldots \ldots$

2. FUNDAMENTALS OF SUPERCONDUCTIVITY AND JOSEPHSON EFFECT . . 4

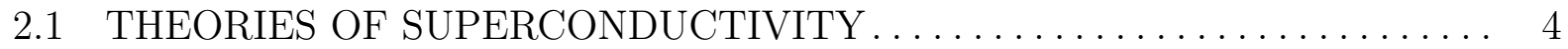

2.2 SURFACE IMPEDANCE OF SUPERCONDUCTORS $\ldots \ldots \ldots \ldots \ldots \ldots \ldots . \quad 8$

2.3 RESIDUAL RESISTIVITY IN SRF CAVITIES $\ldots \ldots \ldots \ldots \ldots \ldots \ldots \ldots \ldots$

2.4 GRAIN BOUNDARIES AS JOSEPHSON JUNCTIONS . . . . . . . . . . 11

2.5 JOSEPHSON EFFECT $\ldots \ldots \ldots \ldots \ldots \ldots \ldots \ldots \ldots \ldots \ldots \ldots \ldots \ldots \ldots \ldots \ldots \ldots$

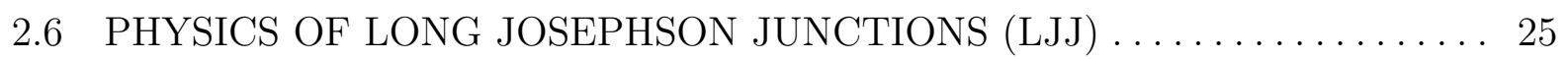

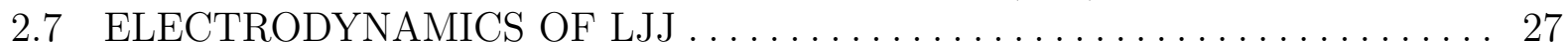

2.8 SOLUTIONS OF SINE-GORDON (SG) EQUATION $\ldots \ldots \ldots \ldots \ldots \ldots \ldots \ldots$

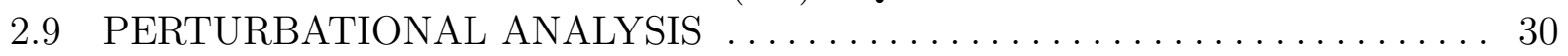

2.10 DYNAMICS OF SINGLE FINITE LENGTH LJJ . . . . . . . . . . . 31

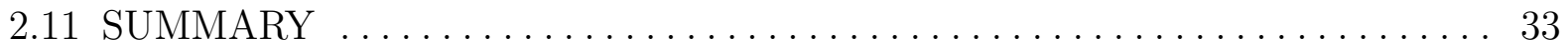

3. SIMULATION OF NONLINEAR DYNAMICS OF JOSEPHSON VORTICES IN

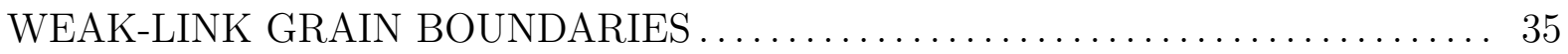

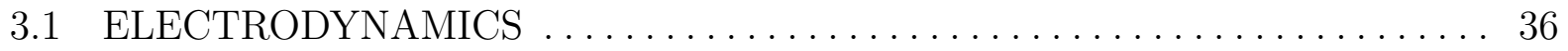

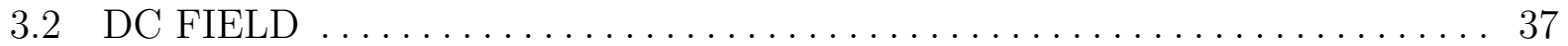

3.3 SINGLE-MODE AC FIELD $\ldots \ldots \ldots \ldots \ldots \ldots \ldots \ldots \ldots \ldots \ldots \ldots \ldots \ldots$

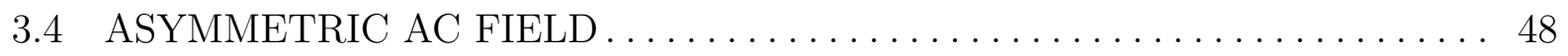

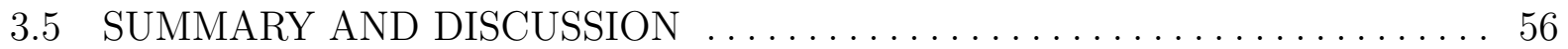

4. VORTEX DYNAMICS IN STRONGLY-COUPLED GRAIN BOUNDARIES AND

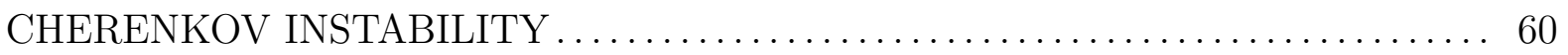

4.1 MECHANISMS OF NONLOCALITY $\ldots \ldots \ldots \ldots \ldots \ldots \ldots \ldots \ldots \ldots \ldots \ldots$

4.2 ELECTRODYNAMICS OF NONLOCAL JUNCTIONS . . . . . . . . . . 61

4.3 PINNING OF J AND AJ VORTICES . . . . . . . . . . . . . . . . 62

4.4 CHERENKOV RADIATION OF MOVING VORTEX . . . . . . . . . . . 63

4.5 VORTEX DYNAMICS IN DIFFERENT NONLOCAL GEOMETRIES . . . . . 63

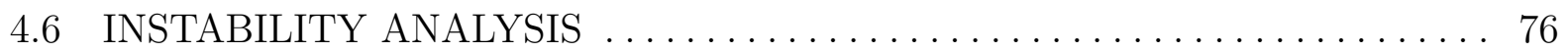

4.7 GENERATION OF QUASIPARTICLES BY A MOVING VORTEX . . . . . 77 
4.8 POWER GENERATED BY A MOVING JOSEPHSON VORTEX . . . . . . 79

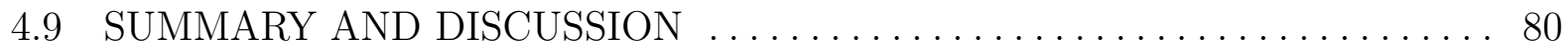

5. VORTEX DYNAMICS IN FINITE LENGTH POLYCRYSTALLINE THIN FILM

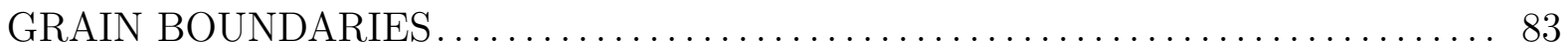

5.1 ELECTRODYNAMICS OF FINITE JUNCTION IN NONLOCAL LIMIT . . 83

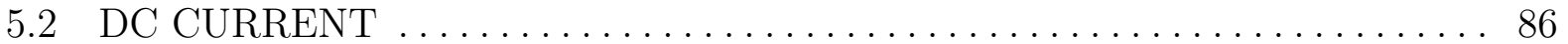

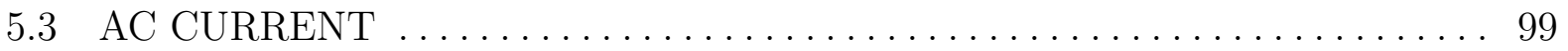

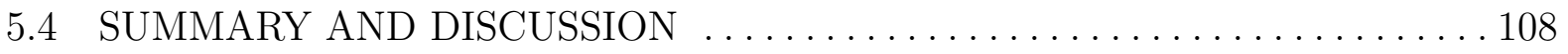

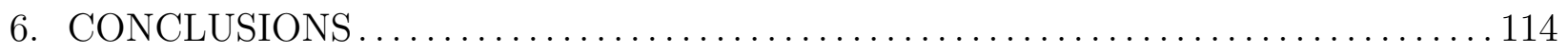
APPENDICES

A. DERIVATION OF NONLOCAL FINITE JUNCTION MAIN EQUATION . . . 125

B. NUMERICAL METHODS ............................... 127 


\section{LIST OF TABLES}

Table 


\section{LIST OF FIGURES}

Figure

Page

1. Magnetic behavior of type-I and type-II superconductors. In type-I superconductors, all magnetic flux enters at thermodynamical critical field while in type-II materials flux penetration starts at lower critical field (read "superheating field") in form of flux quantums and destroys the superconducting state completely at

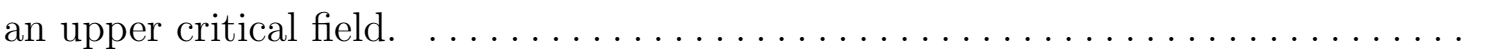

2. The typical structure of a single-cell cavity resonating in $\mathrm{TM}_{010}$ mode where the electric field is parallel to the beam and maximum on the axis while the magnetic field is perpendicular to beam particle and is maximum at the equator. . . . . . 11

3. (a) electron microscopy of dislocation cores in a $7^{\circ}$ [001]-tilt grain boundary YBCO (taken from Ref.[1]) (b) Sketched diagram of a grain boundary formed along a continuous chain of dislocations in crystal lattice.

4. Current-voltage characteristic of a $2.3-\mu \mathrm{m}$-wide bridge straddling a $24^{\circ}$ [001]-titl grain boundary in a 120-nm thick YBCO film. Figure is taken from Ref.[2]. . . . . 13

5. (a) EBSD map showing the grain boudary structure for $\mathrm{Nb} / \mathrm{Cu}$ film and (b) SEM micrograph of the grain boudary structure in $\mathrm{Nb}$ film. Images taken from Ref.[3].

6. Left: $\mathrm{SEM}$ image of the $\mathrm{Nb}_{3} \mathrm{Sn}$ surface. Right: Image of a cross section of $\mathrm{Nb}_{3} \mathrm{Sn}$

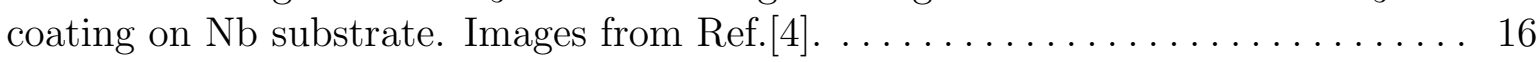

7. A Josephson junction constructed by a thin layer of insulator (I) sandwiched between two superconducting electrodes (S). Overlap of each electrode's superconducing wave function results in small supercurrent tunneling through the insulating barrier. A phase difference is maintained by applying the external voltage

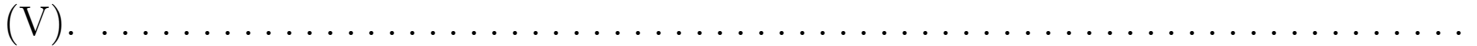

8. Different types of Josephson junction can be constructed changing the weak-link barrier. Figure shows weak-links provided by an insulator (SIS), normal metal

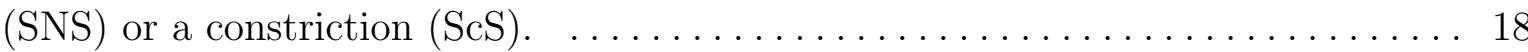

9. A real small Josephson junction can be modeled as an equivalent RCSJ circuit where an ideal junction is shunted by a capacitor and resistor. . . . . . . . . .

10. A tilted washboard potential can analogously model the motion of guage-invariant Josephson phase as a damped particle. The sinusoidal potential is tilted due to

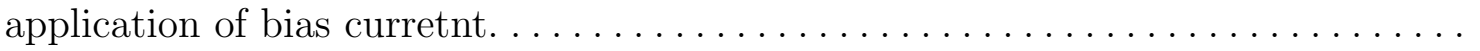


11. Current-voltage characteristics of an overdamped (a) and underdamped (b) junciton. The hysteretic effects in underdamped junctions result in jumpwise transi-

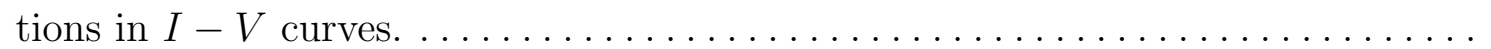

12. Thermal fluctuations result in smearing of the $I-V$ curve in overdamped junction (a), while it reduces the hysteresis in an underdamped junction (b). Here $u=$

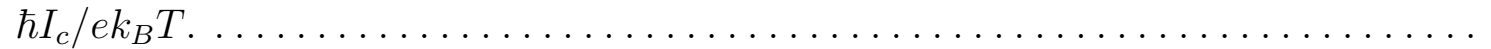

13. Shapiro current-voltage steps in $I-V$ curves of overdamped (a) and underdamped

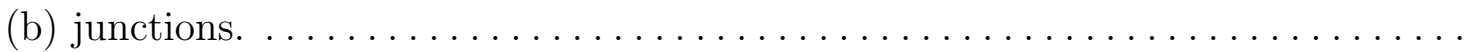

14. Diagram of an extended junction (yellow). Spatial variations of phase difference along the juntion due to applied magnetic field can be calculated using the contours $C_{L}$ and $C_{R}$. The light colored region shows the extent of magneitc field

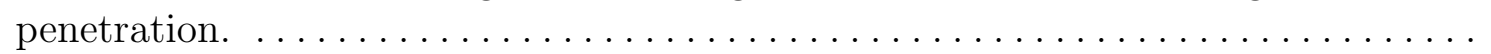

15. Dependence of the junction maximum supercurrent on enclosed flux. Fraunhofer and Airy diffraction patterns for rectangular and circular junctions, respectively.

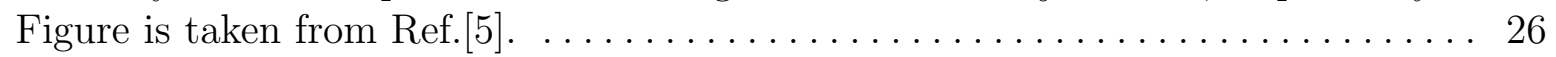

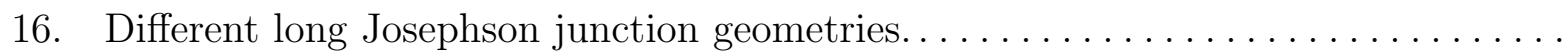

17. Phase and group velocity curves of electromagnetic waves propagation obtained from dispersion relation of a long Josephson junction described by $(45) \ldots \ldots \ldots$. .

18. Josephson vortex represented in terms of phase difference distribution $\theta(x)$, magnetic field distribution $\theta_{x}(x)$ and current distribution $I_{s}(x)=I_{c} \sin \theta(x)$, moving with different velocities.

19. Geometry of a long Josephson junction in a film which screens the uniform magnetic field $H$ applied in the region $x<0$.

20. Profiles of the local magnetic field $B(x)$ in a LJJ at $L=20 \lambda_{J}$ solution of (61)

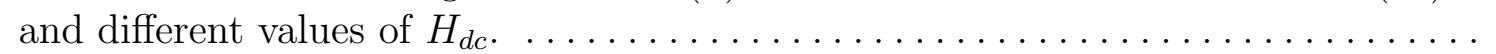

21. Profiles of the local magnetic field $B(x)$ in a LJJ at $L=20 \lambda_{J}, \eta=1.4$ and

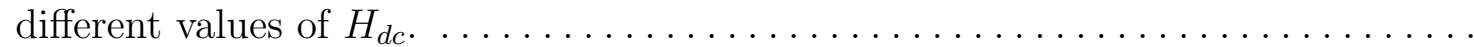

22. Evolution of the local magnetic field $B(x, t)$ along the LJJ, and the instant power $P(t)$ calculated for $H_{d c}=2 H_{1}$. Results of solution of $(58)$ in the overdamped

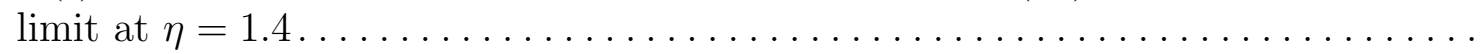


23. Evolution of the local magnetic field $B(x, t)$ along the LJJ, and the instant power $P(t)$ calculated for $H_{d c}=2 H_{1}$. Results of solution of 58 for a moderately dissipative case of $\eta=0.3$. Standing electromagnetic waves generated by moving vortices in the LJJ manifest themselves in "ripple" on $B(x, t)$ and in a more complex behavior of $P(t)$ than for the overdamped limit.

24. Evolution of the local magnetic field $B(x, t)$ calculated for a weakly dissipative case of $\eta=0.01$ at $H_{d c}=1.2 H_{1}$. Vortices undergo multiple reflections from the edges with the reversal of their polarity and velocity. . . . . . . . . . . .

25. Averaged power $\bar{P} / P_{0}$ as a function of reduced dc magnetic field $H_{d c} / H_{1}$ calculated for $\eta=0.2$. Inset shows the flux flow resistance as a function of $H_{d c} / H_{1}$ where $R_{0}=R_{j} / L$ is the total quasiparticle resistance of the junction. $\ldots \ldots \ldots$

26. Snapshots of magnetic field profiles $B(x)$ calculated for $\alpha=0.01$ at different times: (a) just before the first vortex penetrates; (b) just after the penetration of the first vortex/anti-vortex occurred. Arrows show whether the applied field $H(t)$ is increasing or decreasing. . . . . . . . . . . . . . . . . .

27. Evolution of the local magnetic field $B(x, t)$ and the instant dissipated power calculated from (62) for $H_{a c}=2 H_{1}$. Overdamped limit at $\alpha=0.01$. Arrows show the points of annihilation of vortices and antivortices. $\ldots \ldots \ldots \ldots \ldots$

28. Evolution of the local magnetic field $B(x, t)$ and the instant dissipated power calculated from (62) for $H_{a c}=2 H_{1}$. Moderately overdamped limit at $\alpha=0.01$ and $\beta=10$. The flux dynamics is similar to Fig. 27 except the additional "ripples" on $B(x, t)$ due to electromagnetic waves generated because of the effect

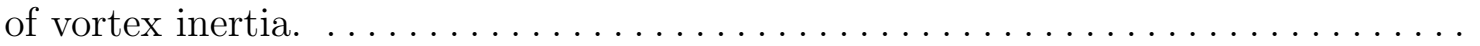

29. Evolution of the local magnetic field $B(x, t)$ at $H_{a c}=2 H_{1}$, and $\alpha=0.01$ for different values of $\beta$ : (a) $\beta=100$; (b): $\beta=1000$. In both cases the ripples on $B(x, t)$ is due to standing electromagnetic waves generated by accelerating/decelerating vortices. In a weakly dissipative case shown in (b), vortex/anti-vortex bundles form during each half cycle.

30. (a) Plots of $\bar{P}\left(H_{a c}\right)$ for different dimensionless frequencies $\alpha=\omega / \omega_{c}=0.01,0.1$ and 1 , in the overdamped limit. Steps in $\bar{P}\left(H_{a c}\right)$ are associated with the addition of vortices to the junction. (b) The surface resistance $R_{s}\left(H_{a c}\right)$ for different $\alpha$. . .

31. Plot of $\bar{P}(\omega)$ at $H_{a c}=4 H_{1}$. The dashed line shows a square root function. . . . 50

32. Frequency dependence of the threshold magnetic field $H_{p}(\omega)$ in the overdamped limit. 
33. Evolution of $B(x, t)$ along the junction during the ac period in the overdamped limit calculated for $\alpha=0.01, H_{a c}=2 H_{1}$ and different dc fields specified on the

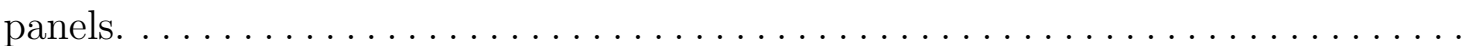

34. $R_{s}$ dependence on $H_{a c}$ for a long, finite junction when $\alpha=0.1$ in presence of different values of dc field. When $H_{a c} \rightarrow H_{d c}$ the ac resistance approaches the asymptotic value of $R_{0}$.

35. Evolution of $B(x, t)$ for a $2 \pi$ period of the bi-harmonic magnetic field. (a): $m=2$, $H_{a 2} / H_{a 1}=0.66$ and $\alpha=0.01$. Changing $\vartheta$ from 0 to $2 \pi / 3$ decreases the number of vortices and increases the number of anti-vortices in the junction. (b): $m=3$, $H_{a 2} / H_{a 1}=0.66$ and $\alpha=0.01$. Varying $\vartheta$ affects flux dynamics but does not result in the vortex/antivortex imbalance.

36. Plots of the average dissipated power $\bar{P}$ as a function of second mode amplitude $H_{a 2}$, for different values of relative phase $\vartheta$ while $\alpha=0.05$ and the fundamental mode is kept constant at $H_{a 1}=2 H_{1}$. (a) $m=2$. (b) $m=3 \ldots \ldots \ldots \ldots \ldots$

37. Plots of the average power $\bar{P}$, as a function of the relative phase $\vartheta$, for $\alpha=0.05$, fixed total field magnitude $\left(H_{a 1}^{2}+H_{a 2}^{2}\right)^{1 / 2}=3 H_{1}$ and different values of $H_{a 2}$ : (a) $m=2$ and $(\mathrm{b}) m=3$. The solid horizontal line at $\approx 2.79$ in both plots shows $\bar{P}$ at $H_{a 1}=3 H_{1}$ and $H_{a 2}=0$.

38. Geometries of long Josephson junctions for which calculations were performed. (a) bulk junction, (b) thin film edge junction, (c) thin film overlap junction. The yellow line shows the Josephson contact, and gray depicts regions of circulating screening currents in the direction perpendicular to the contact. Current stream-

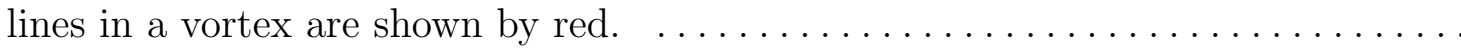

39. A sequence of steady state phase profiles in a propagating vortex (shifted horizontally for clarity in the moving frames) calculated for a bulk junction by solving (86) with $\eta=0.05, \lambda_{J} / \lambda=10$ and for different values of $\beta$. At $\beta_{s}=0.6676$ the peak amplitude of Cherenkov wave reaches $\theta_{c}=8.76$ and starts growing and evolving into an expanding vortex-antivortex pair.

40. Snapshots of $\theta(x)$ at $\beta=\beta_{s}=0.6676$ in a bulk junction of $\eta=0.05$ and $\lambda_{J} / \lambda=10$ which show first three dissociations of the unstable vortex into vortex-antivortex pairs accompanied by Cherenkov radiation. Movies showing the initial stage of vortex instability and formation of the phase pile after multiple generations of vortex-antivortex pairs are available in Ref. [140]. Notice that $\theta(\infty)-\theta(-\infty)=$ $2 \pi$ remains fixed by the phase difference in the initial vortex. . . . . . . . 
41. A 3D image of the evolution of phase pile triggered by an unstable vortex. The dynamic phase distribution $\theta(x, t)$ was calculated from (86) for a bulk junction at $\beta=0.6676, \lambda_{J} / \lambda=10$ and $\eta=0.05$. Here the maximum phase $\theta_{m}(t)$ increases approximately linear with time while the edge vortices move with constant velocities close to $c_{s}$. Individual vortices and antivortices clearly visible at the edges of the expanding phase pile overlap strongly toward its central part. . . . . . . .

42. A snapshot of the normalized magnetic field $B(x, t) / B_{1}$. Here $B(x, t)$ was calculated from (86) for a bulk junction at $\eta=0.05, \beta=0.6676, \lambda_{J} / \lambda=10$ and $B_{1}=\Phi_{0} / 2 \pi \lambda \lambda_{J}$. Inset shows the corresponding phase distribution, $\theta(x, t)$. One can clearly see a complex structure of the left leading edge comprised of a vortex overlapping with a vortex-antivortex pair. Away from the edges vortices overlap so strongly that the Cherenkov radiation gets suppressed almost to zero, and the smooth distribution of $B(x, t)$ in the growing resistive domain can be regarded as a giant multiquanta vortex-antivortex dipole. $\ldots \ldots \ldots \ldots \ldots \ldots \ldots \ldots$

43. Phase profiles before and after instability calculated for a bulk junction with $\eta=0.1$ and $\lambda / \lambda_{J}=\sqrt{10}$. (a) profiles of $\theta(x-v t)$ in a uniformly moving stable vortex at different values of $\beta$ up to $\beta=0.605$. (b) At $\beta_{s}=0.61$ the highest maximum of Cherenkov waves reaches a critical value of $\theta_{c}=8.79$ and starts growing and developing an expanding vortex-antivortex pair. Depicted are the snapshots of the first three splittings of the unstable vortex into vortex-antivortex

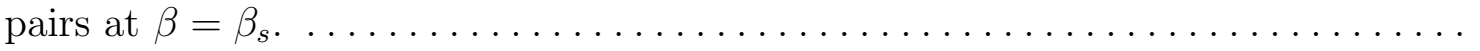

44. A 3D image of vortex instability and the initial stage of the phase pile formation calculated at $\beta=\beta_{s}=0.61$ for a high- $J_{c}$ bulk junction with $\lambda / \lambda_{J}=\sqrt{10}$ and $\eta=0.1$. Here $\theta(x, t)$ in the vortex at $\beta=\beta_{s}$ was computed by solving the full dynamic equation in (86) with the initial distribution equal to the stable singlevortex solution $\theta(x-v t)$ calculated at $\beta=\beta_{s}-0.01$. As a result, the vortex then accelerates slightly and becomes unstable, triggering the growth of the phase pile. After multiple generations of vortex-antivortex pairs, vortices at the leading

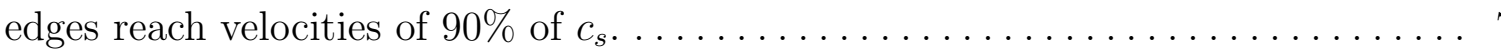

45. A snapshot of the magnetic field $B(x)$ in a phase pile calculated for a bulk high- $J_{c}$ junction at $\beta=\beta_{s}=0.61, \eta=0.1, \lambda / \lambda_{J}=\sqrt{10}$, and $B_{1}=\Phi_{0} / 2 \pi \lambda \lambda_{J}$. The large peaks in $B(x)$ at the leading edges result from emerging vortex-antivortex pairs generating strong Cherenkov waves, the amplitude of which diminishes greatly in the bulk of the dissipative domain where vortices overlap strongly. Inset shows a snapshot of the corresponding phase profile. The growing phase pile with $\theta(x) \gg$ 1 causes small ripples in $B(x)$ due to rapidly oscillating Josephson currents $\propto$ $\sin \theta(x)$ clearly visible in the zoomed structure of the leading edge. 
46. Velocities of a stable singe vortex $v(\beta)$ as functions of current calculated for different junction geometries. The instability occurs at the endpoints (shown by arrows) of the $v(\beta)$ curves. The graph shows $v(\beta)$ for a bulk junction calculated from $(86)$ at $\eta=0.1$ in the seemingly conventional weak-link limit, $\lambda_{J} / \lambda=10$ (for comparison, the blue curve shows $v(\beta)$ calculated from (44) at $\eta=0.1$ ). The magenta and green curves show $v(\beta)$ calculated for a bulk nonlocal junction with $\lambda / \lambda_{J}=\sqrt{10}$ for values of $\eta=0.1$ and $\eta=0.01$, respectively. $\ldots \ldots \ldots \ldots \ldots$

47. A 3D image of vortex instability and the initial stage of the phase pile formation calculated at $\beta=\beta_{s}=0.63$ for a thin film edge junction with $\eta=0.1$ and $l_{J}=\lambda_{P} / 2$. Here $\theta(x, t)$ in the vortex at $\beta=\beta_{s}$ was computed by solving the full dynamic equation in (87) with the initial distribution equal to the stable single-vortex solution $\theta(x-v t)$ calculated at $\beta=\beta_{s}-0.01$. As a result, the vortex then accelerates slightly and becomes unstable, triggering the growth of the phase pile. After multiple generations of vortex-antivortex pairs, vortices at the leading edges reach velocities of $1.12 l_{J} \omega_{J} \ldots \ldots \ldots \ldots \ldots \ldots \ldots \ldots \ldots$

48. Vortex velocity $v(\beta)$ as a function of driving current $\beta=J / J_{c}$ calculated at $\eta=0.1$ for different geometries: nonlocal bulk junction with $\lambda / \lambda_{J}=\sqrt{10}$ (blue), thin film edge junction with $l_{J}=\lambda_{P} / 2$ (red) and thin film overlap junction with $l_{J}=W$ (green). For small currents $(\beta<0.05)$, the velocity $v(\beta)$ in all cases is mostly limited by the ohmic viscose drag while the Cherenkov radiation friction

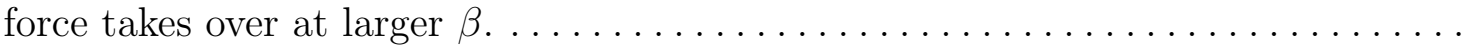

49. The graph shows results for a thin film edge junction in the extreme nonlocal limit described by (80). Notice that both the $v(\beta)$ curves and the critical values $\beta_{s}$ at $\eta=0.1$ and $\eta=0.01$ for the thin film junction are close to those for the nonlocal bulk junction shown in Fig. 46. This is because for a nonlocal bulk junction, $\theta^{\prime \prime}(u)$ in (75) has a sharp peak of width $\sim\left(\lambda_{J} / \lambda\right)^{2} \lambda=0.1 \lambda$ so $G\left[\lambda_{J}(x-u) / \lambda\right]=$ $\pi^{-1} K_{0}\left[\lambda_{J}|x-u| / \lambda\right]$ can be approximated by its expansion at small argument, $K_{0}\left(\lambda_{J} x / \lambda\right) \rightarrow \ln \left(2 \lambda / \lambda_{J}|x|\right)-0.577$, which reduces to $(79)$. Here any constant factor under the log does not affect $\theta(x, t)$ since $\theta^{\prime}(-\infty)=\theta^{\prime}(\infty)=0 \ldots \ldots \ldots$

50. A 3D image of vortex instability and the initial stage of formation of phase pile at $\beta=\beta_{s}=0.63$ in an overlap junction at $\eta=0.1$ and $l_{J}=W$. Here $\theta(x, t)$ in the vortex at $\beta=\beta_{s}$ was computed by solving the full dynamic equation in (89) with the initial distribution equal to the stable single-vortex solution $\theta(x-v t)$ calculated at $\beta=\beta_{s}-0.01$. As a result, the vortex then accelerates slightly and becomes unstable, triggering the growth of the phase pile. After generation of many vortex-antivortex pairs, the leading edges of the phase pile reach the

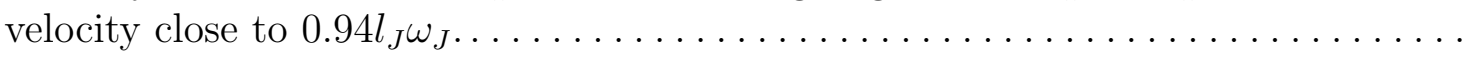

51. Geometries of a Josephson junction in a thin film with the vortex (a) parallel and (b) perpendicular to the broad face of the film. . . . . . . . . . . . . 
52. $\theta(x)$ in a static vortex calculated from (106) for different values of $\epsilon$ as described

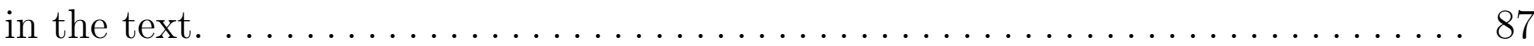

53. Penetration of single vortices in an overdamped junction with $\eta=2$ and $\beta_{0}=1.05$ calculated for $k=0.02$ and $\epsilon=2 \cdot 10^{-3} \ldots \ldots \ldots \ldots \ldots \ldots \ldots$

54. Snapshots of moving vortices in the middle of the junction calculated from (106) for different currents at $\eta=2, k=0.02$ and $\epsilon=2 \cdot 10^{-3} \ldots \ldots \ldots \ldots \ldots \ldots$

55. Upper and lower limits between which $\theta(x, t)$ oscillates, calculated for $\eta=2$ and $\beta_{0}=7$. The red curve shows $\theta_{s}(x)$ described by $(113) \ldots \ldots \ldots \ldots \ldots \ldots$

56. The dc voltage $\bar{V}=\langle V(t)\rangle$ calculated from (114) for different values of $\eta$, where $\langle\ldots\rangle$ denotes time averaging, and $V_{0}=\Phi_{0} \omega_{J} / 2 \pi \ldots \ldots \ldots \ldots \ldots \ldots \ldots \ldots \ldots \ldots$

57. A wake radiated behind the moving vortex at $\eta=1$ and $\beta_{0}=0.995$. Here the vortex gets fully reflected from the edge as antivortex. $\ldots \ldots \ldots \ldots \ldots \ldots \ldots$

58. Snapshots of dynamic patterns formed by counter-moving vortices and antivortices calculated for $\eta=1, \epsilon=2 \cdot 10^{-3}, k=0.02$, (a) $\beta_{0}=1.05$ and (b) $\beta_{0}=1.09$. Different colors correspond to different times $t$ during the time period after which the phase structures repeat themselves periodically after shifting up in $\theta$. As current further increases, the patterns shown in Figs. 58 a and b gradually turn into a phase slip profile similar to that is shown in Fig. 55. The asymmetry of the profiles $\theta(x, t)$ with respect to $x=0$ is due to the effect of the gradient in $\beta(x) .93$

59. Initial state of generation of v-av pairs calculated at $\eta=0.7$ and $\beta_{0}=0.995$. The Cherenkov splitting instability of a vortex occurs right after it enters the junction and ultimately results in the dynamic pattern similar to those shown in Fig. 58. . 95

60. Dissipation power vs de current calculated for different damping constants shows a quadratic behavior at currents well above the threshold of penetration of a vortex. 95

61. Spectrum of Fourier components of voltage $V(t)$ calculated for $\eta=0.8$ and different currents corresponding to different number of vortices in the junction (left panel). Right panel shows the de voltage $\bar{V}(\beta)$ in which jumps result from the

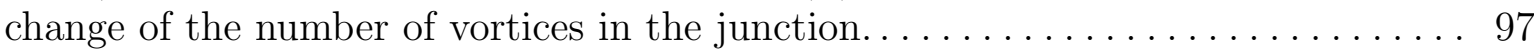

62. A vortex depinned from the defect at the left edge of junction accelerates and produces a vortex-antivortex pair at $x \approx 0.1$ after the next vortex enters the junction. Simulations were done for $\delta_{0}=0.5, \beta=0.8$ and $\eta=0.3 \ldots \ldots \ldots$. . 99

63. A vortex accelerates as it approaches the defect in the center and decelerates once it passes the defect in the case of $\zeta=0.01, \delta_{0}=0.15, \beta_{0}=0.98$, and $\eta=1 \ldots 100$ 
64. At $\eta=0.7$ even a weak defect can accelerate the approaching vortex so that it produces a critical radiation wake which generates a v-av pair. Figure shows the dynamics of a vortex in the absence (left) and the presence (right) of a defect

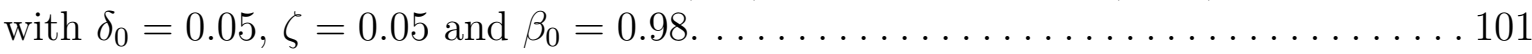

65. Interaction of a vortex penetrating from left with a v-av pair produced simultaneously by a weak defect with $\delta_{0}=0.2$ and $\zeta=0.01, k=0.1$ and $\eta=1$ at the threshold current $\beta_{0}=0.98$. The vortex which entered from the left edge annihilates with the antivortex produced at the defect while the remaining vortex exits

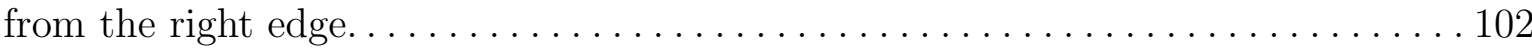

66. Oscillatory dynamics of vortices in an overdamped junction with $\eta=2$ at the penetration threshold $\beta_{0}=1.237$. The vortex enters the junction during the positive cycle of $\beta(t)$, stops midway when $\beta(t)=0$, turns around and exits through the edge during the negative cycle of $\beta(t) . \ldots \ldots \ldots \ldots \ldots \ldots \ldots \ldots$

67. Ballistic penetration of vortices and antivortices into an overdamped junction with $\eta=2$ at $\beta_{0}=1.245$. Here vortices and antivortices traverse the junction and exit from the other end. Notice that the moving vortex extends nearly over the entire junction and produces no visible radiation. . . . . . . . . . . . . 104

68. Fourier spectrum of voltage at $\eta=2$ calculated for oscillatory vortex dynamics at $\beta_{0}=1.237$ and ballistic vortex penetration at $\beta_{0}=1.245$ represented in Figs. 66 and 67 , respectively. The peaks in $V_{\omega}$ occur at the multiples of the ac frequency $\omega_{n}=n \omega$, where $\omega=\pi / 30$ and $n=1,2,3, \ldots$. Notice that voltage harmonics with even $n$ disappear as the vortex dynamics changes from oscillatory to ballistic. 105

69. AC power plots $P\left(\beta_{0}\right)$ for different damping constants. At large currents $\beta_{0} \gtrsim 3$, the curves $P\left(\beta_{0}\right)$ approach the ohmic limit $P=P_{0} \beta_{0}^{2} / 2 \eta \ldots \ldots \ldots \ldots \ldots \ldots \ldots$

70. Each large step in dissipation plots corresponds to an additional vortex penetration during ac current cycles. Shown above is the second vortex partial penetration above a ballistic motion of vortices calculated at $\eta=1$ and $\beta_{0}=1.26 . \quad \ldots 107$

71. Mechanism of vortex penetration into the junction calculated at $\eta=0.7$ for (a) nonlocal model at $\beta_{0}=1.085$ and (b) local at $\beta_{0}=1.01$. Vortices are produced through pair production inside the underdamped junction in thin film nonlocal model.

72. Generation of a v-av pair due to radiation of a penetrating vortex at $\eta=0.7$ at $\beta_{0}=1.092$. The dynamics becomes periodic afterwards which is shown in Fig.73a.110 
73. (a) Generation of a pair as due to excessive radiation produces the spike in Fig.69 for $\eta=0.7$ and $\beta_{0}=1.092$. With increase of current the amplitude of oscillations of pair increases until at (b) $\beta_{0}=1.098$ the vortex of the pair annihilates with left penetrating antivortex and the junction stabilizes after this transient with one vortex and one antivortex penetrating from edges. This drop in number of vortices also causes the power to drop. (c) Further increase of current causes the vortex and antivortex to become so close such that the solution turns into a ballistic penetration of vortex/antivortex at each current cycle. . . . . . . . 111

74. Penetration of radiating vortices and antivortices at $\eta=0.4$ and $\beta_{0}=1.034$

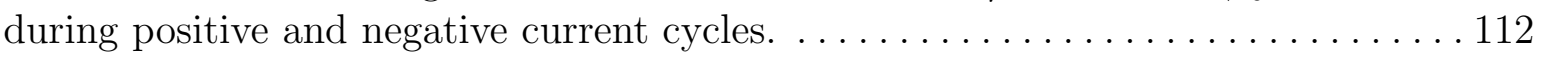

75. Dynamics of $\theta(-d / 2, t)$ and $\theta(d / 2, t)$ at the edges at $\eta=0.2$ and $\beta_{0}=1.1$. Here $\theta(x, t)$ remains nearly uniform along junction, indicating a phase slip behavior. . . 112 


\section{CHAPTER 1}

\section{INTRODUCTION}

High energy accelerator machines use superconducting radio-frequency (SRF) cavities due to their extremely low losses (high quality factors) as well as the ability to produce high accelerating gradients. Hence, the two most important figure of merits, namely high acclerating field $E_{a c c}$ and high quality factor $Q$ decide the best performing SRF cavities. Over the course of fifty years, SRF technology has tremendously evolved, fabricating prototype $\mathrm{Nb}$ cavities able to achieve high gradients $E a c c \approx 50 \mathrm{MV} / \mathrm{m}$ with significant quality factors $Q \approx 10^{10}-10^{11}$ performing almost at the theoretical limit of peak surface magnetic fields $H \simeq 200 \mathrm{mT}$, still at economically efficient low temperatures[6, 7, 8, 9]. The remarkable results were possible by understanding and eliminating many important factors such as field emission and multipacting that used to negatively impact the performance of early $\mathrm{Nb}$ cavities. However, similar to other technological advancements, the state-of-the-art level of performance in $\mathrm{Nb}$ cavities still suffers from traditional and newly emerged degrading factors such as structural defects, trapped magnetic flux, surface impurities, etc., whose contributions were completely ignored at early stages[8, 10]. Some of these performance-limiting effects can be even more pronounced in promising $\mathrm{SRF}$ materials like $\mathrm{Nb}_{3} \mathrm{Sn}[11,12]$ and proposed multilayer coating structures in next generation high performance SRF cavities[13]. SRF technologies and operations take place at extremely low temperatures with significant cryogenic and material costs that cause every bit of losses uneconomical . Moreover, the use of SRF cavities in next generation linear colliders such as ILC (International Linear Collider) with the goal of achieving energies of the range of $\mathrm{TeV}$, repeats the necessity to push the current limits of SRF cavity performance. Therefore, an immediate need is widely recognized among the SRF community to advance our understanding of the physics behind the degrading factors and mechanisms that control the surface and bulk properties of SRF cavities[14].

\subsection{GRAIN BOUNDARIES IN SRF MATERIALS}

Structural defects can impose serious restrictions on performance of SRF cavities either through initiating a thermal breakdown or by increasing the surface resistance, hence 
decreasing the quality factor. Grain boundaries in polycrystalline materials are common planar defects which subdivide the material into single misoriented crystallites with current-impeding properties which results in serious performance-limiting effects, particularly in high- $T_{c}$ cuprates and pnictides where current breaks into single isolated loops inside grains[11]. In polycrystalline superconducting materials, grain boundaries locally depress the superconductivity, hence facilitate penetration of Josephson vortices due to reduced onset field of penetration along the boundary sites[5]. Early vortex penetration can significantly harm the performance of SRF cavities in which the magnitude of screening currents at the inner surface of cavity reach almost the depairing current density[6]. In case of SRF Nb cavities the result of investigations on grain boundaries effects have been inconclusive. There have been experiments[15, 16, 17] which attribute the residual surface resistance of small grain Nb cavities to the effect of grain boundaries while some studies[18, 19, 20] show insignificant dependence of residual resistivity to the size of the grains. The latter can be supported by the fact that some of the best performing $\mathrm{Nb}$ cavities are fabricated from small grain $\mathrm{Nb}$ ingots[21, 22]. The uncertainty in the effect of grain boundaries in Nb may originate from the fact that $\mathrm{Nb}$ is a material in which the size of the cooper pair $(\xi=40 \mathrm{~nm})$ is rather large compared to the interatomic scales of grain boundaries. However, experimental measurements performed on SRF quality high-angle, single and bi-crystal Nb grain boundaries exhibit degraded superconducting properties[23, 24]. Moreover, segregation of impurities around grain boundaries in $\mathrm{Nb}$ can degrade their current transport properties turning a grain boundary into a weak superconducting region[11]. Grain boundaries in other SRF materials like $\mathrm{Nb}_{3} \mathrm{Sn}$ are known to effective pinning centers[11, 12] for vortices which implies their weakened superconducting essence. Therefore, both types of weak and strong link grain boundaries in $\mathrm{Nb}_{3} \mathrm{Sn}$ can be essential degrading factors through trapping and penetration of Josephson vortices.

\subsection{MOTIVATION}

Although certain theoretical attempts have been made to address the effect of grain boundaries in SRF materials[17, 25, 26], the lack of understanding of the physics of Josephson vortex penetration and their mechanisms of dissipation inside the grain boundaries, has certainly been an important source of confusion. In addition, the absence of specific calculations to estimate the amount of power dissipation due to vortex flow in grain boundaries leaves the importance of their contribution to residual surface resistance an open question. 
Moreover, specific geometrical shapes of the cavities, the direction of the applied rf electromagnetic field as well as the type of grain boundaries present, require particular models and calculations to be made in order to evaluate the extent of grain boundary dissipative effects. Therefore, to address these issues we performed extensive numerical simulation of nonlinear dynamics of Josephson vortex penetration in both types of weak-link and strong-link grain boundaries with different geometries in both bulk and thin film applications. The results are certainly applicable to a wide range of problems from flux flow oscillators to superconducting quantum circuits, quantum computing and $\mathrm{THz}$ radiation sources where Josephson junctions are essential components.

\subsection{ORGANIZATION OF DISSERTATION}

The rest of this work is organized as follows: Chapter 2 gives an outline of the basic concepts of superconductivity and Josephson effect. It also discusses some of the details of the Josephson vortex dynamics in different conventional long Josephson junctions. In chapter 3, I present the original result of my simulations of nonlinear dynamics of Josephson vortices in weak-link grain boundaries under dc and ac electromagnetic fields and calculate the power dissipation as functions of field amplitude and frequency. It also shows the result of calculations of onset field for penetration of vortices as function of frequency. Chapter 4 starts off with a discussion of emergence of nonlocality in the electromagnetic response of strong-linked grain boundaries following by the result of simulation of single Josephson vortex dynamics in different nonlocal geometries and prediction of new instability in vortex dynamics due to Cherenkov radiation. The dissertation continues with a presentation of result of numerical simulation of the electromagnetic response of polycrystalline thin film grain boundaries in both parallel and perpendicular directions to the wide surface of film. Finally, I discuss the results of my work and derive conclusions in chapter 6 . 


\section{CHAPTER 2}

\section{FUNDAMENTALS OF SUPERCONDUCTIVITY AND JOSEPHSON EFFECT}

The ability to liquefy Helium for the first time in 1908 by Kamerlingh Onnes gave him the opportunity to measure the electrical properties of metals at extremely low temperatures. Three years later in 1911 he was able to discover superconductivity in solid mercury wire by measuring zero electrical resistance at transition temperature $T_{c}=4.2 \mathrm{~K}$. This is conventionally known as the first hallmark of superconductivity where a superconducting material shows exactly zero electrical resistance below some transition temperature $T_{c}$ and becomes a perfect conductor.

The second hallmark of superconductivity was announced during the work of Meissner and Oschenfield in 1933 where they showed that below $T_{c}$ a superconductor expels magnetic flux from its interior (Meissner effect). In Meissner effect dc magnetic flux will be screened from the superconductor bulk by superconducting screening currents flowing in a thin surface layer of thickness $\lambda$ (London penetration depth), illustrating that a superconductor is not

only a perfect conductor but also a perfect diamagnet. However, perfect diamagnetism can only happen up to a certain critical field $H_{c}$ above which the magnetic field penetrates fully into the superconductor and the superconductivity will be destroyed. In other words at critical magnetic field $H_{c}$, the flowing supercurrent reaches its maximum density $J_{d}$ above which the supercurrent carriers (Cooper pairs) will break up and the superconductor will return to its normal state. This is a more fundamental property of superconductors stating that a superconductor can only carry dc supercurrents with zero resistance up to a critical current density $J_{c}$ known as a material property and was first established in the work of Silsbee[27].

The Mesisner effect in superconductors can well be described by London theory[28] using a two fluid model in which a superconductor is considered to have normal and superconducting charge carriers, one taking over the other in a normal and superconducting state, respectively.

\subsection{THEORIES OF SUPERCONDUCTIVITY}




\subsubsection{MICROSCOPIC BCS THEORY}

More than four decades after the discovery of superconductivity, J. Bardeen, L. Cooper and J. R. Schrieffer (BCS)[29] proposed the first successful microscopic theory that explained superconductivity as a quantum mechanical phenomena. In this outstanding work it was shown that every two electrons near the Fermi surface with equal and opposite momentum and spin can indulge in a weak attractive bonding by exchanging phonons (lattice oscillations) which results in formation of Cooper pairs. The zero net momentum and spin of each pair makes Cooper pairs to be bosons and form a bosonic condensate described by a single quantum mechanical wave function $\psi(r)=\left(n_{s} / 2\right)^{1 / 2} e^{i \varphi(r)}$ where $n_{s}$ is the density of Cooper pairs all having the same phase $\varphi$. It is this phase coherence of all Cooper pairs that gives rise to marvelous properties of a quantum mechanical phenomena like superconductivity. The idea of phase coherence length $\xi$ was first proposed by Pippard and then used by BCS theory to describe the spatial extent over which Cooper pairs overlap and a change in their wave function occurs.

A key feature of the BCS theory was the prediction of appearance of an energy gap $\Delta(T)$ between the ground state of Cooper pairs and their next excited state. It was shown that a minimum energy of $E_{g}=2 \Delta(T)$ is required to break up a pair and form two quasi-particle excitations. The theory relates the energy gap at $T=0$ to the critical temperature by $[5]$

$$
\Delta(0)=1.76 k_{B} T_{c}
$$

suggesting that only electrons within the energy range of $k_{B} T_{c}$ take part in superconductivity. At nonzero temperatures some of the Cooper pairs can break and form quasiparticles in the form of both electron-like and hole-like excitations. At temperatures close to $T_{c}$ the density of Cooper pairs decreases significantly such that the gap energy $\Delta(T) \rightarrow 0$ at transition temperature $T_{c}$.

\subsubsection{LONDON PHENOMENOLOGICAL THEORY}

As mentioned before, the electrodynamics of the two basic properties of superconductors, namely, perfect conductivity and perfect diamagnetism were well presented by London brothers who proposed two equations governing the microscopic electric and magnetic fields

$$
\begin{array}{r}
\frac{\partial J_{s}}{\partial t}=\frac{n_{s} e^{2}}{m} E \\
\nabla \times J_{s}=-\frac{n_{s} e^{2}}{m} B
\end{array}
$$


based on a two fluid model where $n_{s}$ is the density of superconducting electrons with $e$ and $m$ as the charge and mass of electron, respectively[5]. The first equation describes perfect conductivity where electrons accelerate continuously due to electric field since no resistance is present. The second London equation in (3), in combination with Maxwell's equation $\nabla \times B=\mu_{0} J$ results in

$$
\nabla^{2} B=\frac{1}{\lambda^{2}} B
$$

where $\lambda=m / \mu_{0} n_{s} e^{2}$ is London penetration $\operatorname{depth}[5]$. Equation (4) implies that the flux density in superconductor is not only constant as in a perfect conductor but also drops exponentially to zero in the bulk over the screening length of $\lambda$, describing Meissner effect.

\subsubsection{GINZBURG-LANDAU THEORY AND TYPES OF SUPERCONDUC- TORS}

A major approach to classification of superconducting materials has been based on how a superconductor would respond to an external magnetic field. This can be obtained as a byproduct of the more general phenomenological theory of Ginzburg-Landau (G-L) where superconductivity is considered a second order thermodynamical phase transition phenomena[30]. G-L theory introduces a pseudo-wavefunction $\psi$ as an order parameter which describes the local density of superconducting electrons given by[5]

$$
2 n_{s}=|\psi(x)|^{2}
$$

as well as a characteristic length known as GL coherence length $\xi(T)$ which characterizes the distance over which the $\psi(x)$ changes.

In a physical boundary between a superconductor and normal conductor the density of superconducting carriers (Cooper pairs) increase from zero at the boundary to a constant value inside the superconductor within the coherence distance $\xi$. This means that the free energy per unit area is raised by $\mu_{0} H_{c}^{2} \xi / 2$. On the other hand in the presence of magnetic field $H_{e}$ the magnetic flux is allowed over the length $\lambda$ into superconductor lowering the energy per unit area by $-\mu_{0} H_{e}^{2} \lambda / 2$. The net boundary energy per unit area is then $[6]$

$$
\mu_{0}\left(\xi H_{c}^{2}-\lambda H_{e}^{2}\right) / 2
$$

A more accurate treatment of $\mathrm{G}-\mathrm{L}$ equations indicates that the $G$ - $L$ parameter $\kappa$ defined as

$$
\kappa=\frac{\lambda}{\xi}
$$

distinguishes a positive net energy when $\kappa<1 / \sqrt{2}$ as opposed to negative energy when $\kappa>1 / \sqrt{2}$. 


\section{Type-I Superconductors}

In a type-I superconductor the positive surface energy barrier $(\kappa<1 / \sqrt{2})$ inside the superconductor indicates that the magnetic field is screened perfectly up to $H_{c}$ from the interior. At larger magnetic field the phase coherence of Cooper pairs will be destroyed and the superconductor will return back to normal state (Fig.1).

\section{Type-II Superconductors}

A type-II superconductor $(\kappa>1 / \sqrt{2})$ screens out the external magnetic field up to lower critical field $H_{c 1}$. At fields $H>H_{c 1}$ the net surface energy becomes negative indicating that it is energitically favorable to have as much superconducting/normal-conducting boundaries as possible inside the bulk of superconductor. However, flux penetration does not happen up until the current density flowing along the surface reaches the critical value at which the Meissner state becomes unstable at the superheating field, $H_{s}$. Flux penetration takes place in the form of supercurrent loops (Abrikosov vortices[31]) each carrying a quantum of flux $\Phi_{0}=h / 2 e=2.07 \times 10^{-15} \mathrm{~Wb}$, with normal cores of size $\xi$ screened from the rest of superconductor bulk over length $\lambda$. Flux penetration continues on until at a larger upper critical field $H_{c 2}$ the normal cores of vortices overlap and the superconducting sample will turn normal (Fig. 1).

Vortices are small magnetic flux tubes which unless pinned by some inhomogeneity, can move under a transport supercurrent $\mathbf{J}$ due to so called Lorentz force $\mathbf{J} \times \Phi_{0}$ and leave behind a resistive electric field that can cause energy dissipation. Abrikosov also showed that repulsion between vortices with same magnetic polarity will give rise to a triangular vortex lattice with flux density proportional to $H / H_{c 1}$.

\section{High- $T_{c}$ Superconductors}

The discovery of materials with high transition temperatures by Bednorz and Müller[32] in 1986 opened a complete new era in the history of superconductivity. These materials have mainly layered structures dominated by copper oxide planes which are known to be responsible for superconductivity. So far, a superconducting transition temperature as high as $163 \mathrm{~K}$ has been reported in the $\mathrm{Hg}$-based $\mathrm{HgBa}_{2} \mathrm{Ca}_{2} \mathrm{Cu}_{3} \mathrm{O}_{8}$ compound at a high external pressure. This is a great achievement in terms of cryogenic applications since these materials can be cooled down well below their transition temperature with liquid nitrogen which is readily accessible and more cost efficient material than liquid helium. However, certain 

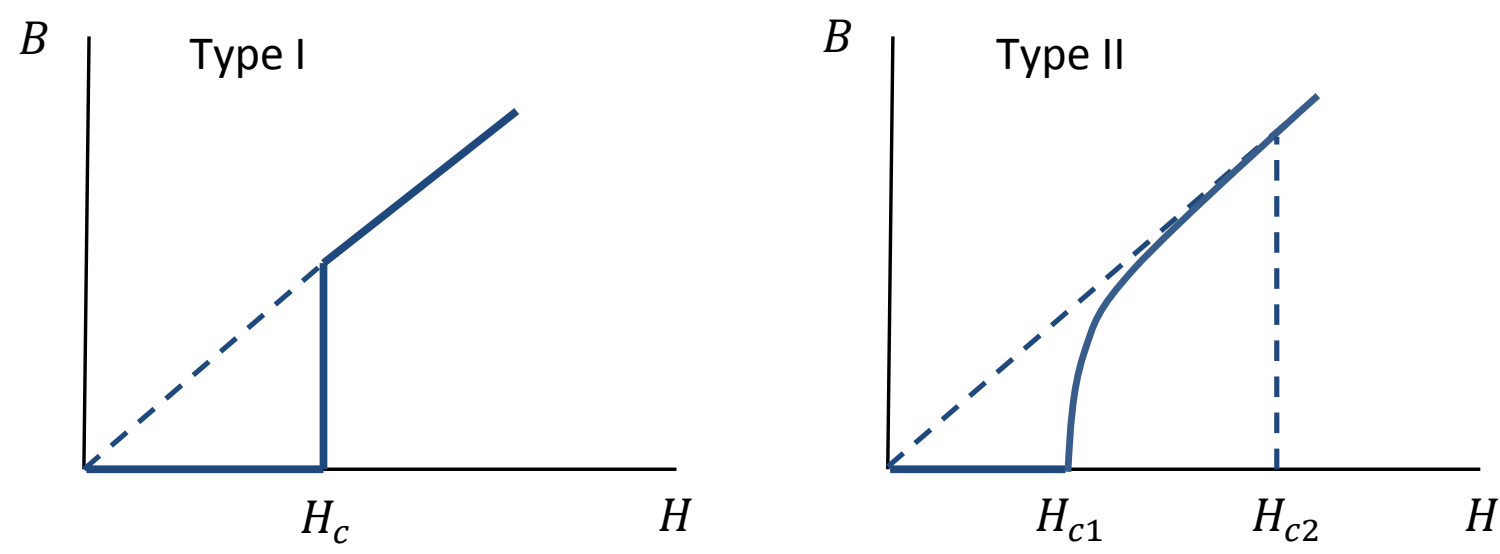

FIG. 1: Magnetic behavior of type-I and type-II superconductors. In type-I superconductors, all magnetic flux enters at thermodynamical critical field while in type-II materials flux penetration starts at lower critical field (read "superheating field") in form of flux quantums and destroys the superconducting state completely at an upper critical field.

structural properties of High- $\mathrm{T}_{c}$ materials make them hard to fabricate which has greatly reduced their immediate applications. Operation at high temperatures, low electron density and high anisotropy cause them to be more prone to thermal fluctuations which enhances the possibility of losing the zero electrical resistance state. Although it is more or less clear that the superconductivity is caused by pairing of electrons but the nature of pairing has been in great controversy, therefore, the field is still suffering from absence of a comprehensive microscopic theory explaining the nature of superconductivity[5].

\subsection{SURFACE IMPEDANCE OF SUPERCONDUCTORS}

When an electromagnetic plane-wave is incident normally on the surface of a conductor, it induces surface currents within the skin depth of the conductor which shield the electric and magnetic fields from the bulk of the metal. In analogy with dc resistance, the surface impedance $z_{s}$ is defined by the ratio of electric field at the surface to the total current flowing in the conductor. From Maxwell's equations this is equivalent to the ratio of surface electric and magnetic fields, therefore, $z_{s}$ is given by

$$
Z_{s}=\frac{E}{H}=\sqrt{\frac{i \mu_{0} \omega}{\sigma}}=R_{s}+i X_{s}
$$

where $\omega$ is the angular frequency of the plane wave, $\sigma=\sigma_{0} /(1-i \omega \tau)$ is complex conductivity of the conductor, $\sigma_{0}$ is the dc conductivity and $\tau$ is electrons scattering time. The real part $R_{s}$ gives the ac surface resistance and the imaginary part $X_{s}$ gives the surface reactance. 
Nonzero reactance means that the surface electric field is not in phase with the total current due to the rate of change of magnetic flux in the conductor. If $\omega \tau \ll 1$, the surface resistance of a conductor is obtained by

$$
R_{s}=\sqrt{\frac{\mu_{0} \omega}{2 \sigma_{0}}} .
$$

In two fluid model the total current flowing in a superconductor is composed of a supercurrent component carried by superconducting electrons with density $n_{s}\left(n_{s} \rightarrow 0\right.$ as $\left.T \rightarrow T_{c}\right)$ and a normal current component with carrier density $n_{n}\left(n_{n} \rightarrow 0\right.$ as $\left.T \rightarrow 0\right)$. Consequently, in analogy with normal conductors, the total conductivity is the sum of the normal and superconducting parts, $\sigma=\sigma_{n}+\sigma_{s}$. Substituting for ac supercurrent $J_{s 0} e^{i \omega t}$ into first London equation, we have

$$
J_{s}=-i \sigma_{s} E
$$

where $\sigma_{s}=n_{s} e^{2} / m \omega$. The pure imaginary relation between current and field implies a lossless ac supercurrent as expected. Now the total two fluid current becomes

$$
J=\left(\sigma_{n}-i \sigma_{s}\right) E
$$

which in analogy with the treatment of normal conductors, the surface impedance of superconductor is given by

$$
Z_{s}=\sqrt{\frac{i \mu_{0} \omega}{\sigma_{n}-i \sigma_{s}}} .
$$

In order to derive a simpler expression we consider that at low temperatures $T \ll T_{c}$ where $n_{n} \ll n_{s}$ and also for microwave frequencies where $\tau \ll 1 / \omega$, the value of $\sigma_{n}$ is much less than $\sigma_{s}$. Therefore, one can show that for usual definition of surface impedance $Z_{s}=R_{s}+i X_{s}$ the real and imaginary parts is given by[33]

$$
\begin{array}{r}
R_{s}=\frac{\mu_{0}^{2}}{2} \sigma_{n} \omega^{2} \lambda^{3} \\
X_{s}=\mu_{0} \omega \lambda .
\end{array}
$$

The surface resistance in superconductor is proportional to $\omega^{2}$ in contrast to normal conductor where $R_{s} \propto \omega^{1 / 2}$. In superconductors, this surface resistance come from the imperfect shielding of normal electrons from the alternating electromagnetic fields due to the inertia of cooper pairs. As a result a nonzero time varying electric field at the surface of superconductor can accelerate and decelerate normal electrons, leading to power dissipation and surface resistance. Moreover, as BCS predicts that $\sigma_{n} \propto e^{-\Delta / k_{B} T}$, it shows that $R_{s} \rightarrow 0$ at $T=0$. However, experiments indicate that a temperature-independent residual resistance 
$R_{i}$ is present even at $T=0$ and there is a dependency of surface resistance to rf current amplitude which cannot be explained within the current microscopic theories. A semi-empirical relation for $T<T_{c} / 2$ based on numerous experiments has been derived for surface resistance given by

$$
R_{s}=\frac{A}{T} \omega^{2} \exp \left(\frac{-\Delta(T)}{k_{B} T}\right)+R_{i}
$$

where $A$ is mostly material dependent[34], the first term is known as $R_{B C S}(T)$ and second term describes the residual surface resistance.

\subsection{RESIDUAL RESISTIVITY IN SRF CAVITIES}

A major application of superconducting materials is in Superconducting Radio-Frequency (SRF) cavities (Fig. 2) in accelerators where strong electromagnetic fields are used to accelerate a beam of charged particles to highest energies possible. This was only applicable due to extremely low rf losses in cavities superconducting walls. The power dissiapted is quantified by the quality factor $Q$, inversely proportional to surface resistance $R_{s}$. In low temperature applications as in SRF cavities the operating temperature is normally chosen as low as economically efficient. In this range of temperatures, the temperature dependent component of surface resistance, $R_{B C S}(T)$ is exponentially decaying with the decrease of temperature and is small enough so that the contribution of residual resistance is significant and becomes a major source of power dissipation. For instance, for a $1.5 \mathrm{GHz}$ cavity operating at $2 \mathrm{~K}$, the BCS resistance is $\sim 20 \mathrm{n} \Omega$, while for a well prepared niobium the residual resistivity can reach $10-20 \mathrm{n} \Omega[6]$.

The physics behind the residual resistance $R_{i}$ is not well understood however, many extrinsic mechanisms have been pointed out. Surface contamination with lossy impurities such as oxides and metallic hydrides can cause major decrease of the quality factor (Q-disease) [6, 7]. Trapped magnetic flux in the form of vortices, either due to ambient magnetic field or due to thermo-electric currents during cavity cool down, can couple to the rf electromagnetic fields and oscillate rapidly causing major power dissipation[6, 8, 10]. The existence of grain boundaries in polycrystalline materials such as niobium and $\mathrm{Nb}_{3} \mathrm{Sn}$ used in fabrication of SRF cavities can increase the flux dissipation both by providing effective pinning centers for trapped vortices as well as facilitating penetration of new vortices due to their current-blocking properties[15, 16, 25, 35]. The existence of subgap energy states has also considered as an intrinsic mechanism which results in residual resistance[36, 37]. Since screening currents are only flowing over a thin layer of cavity inner surface, it is important 


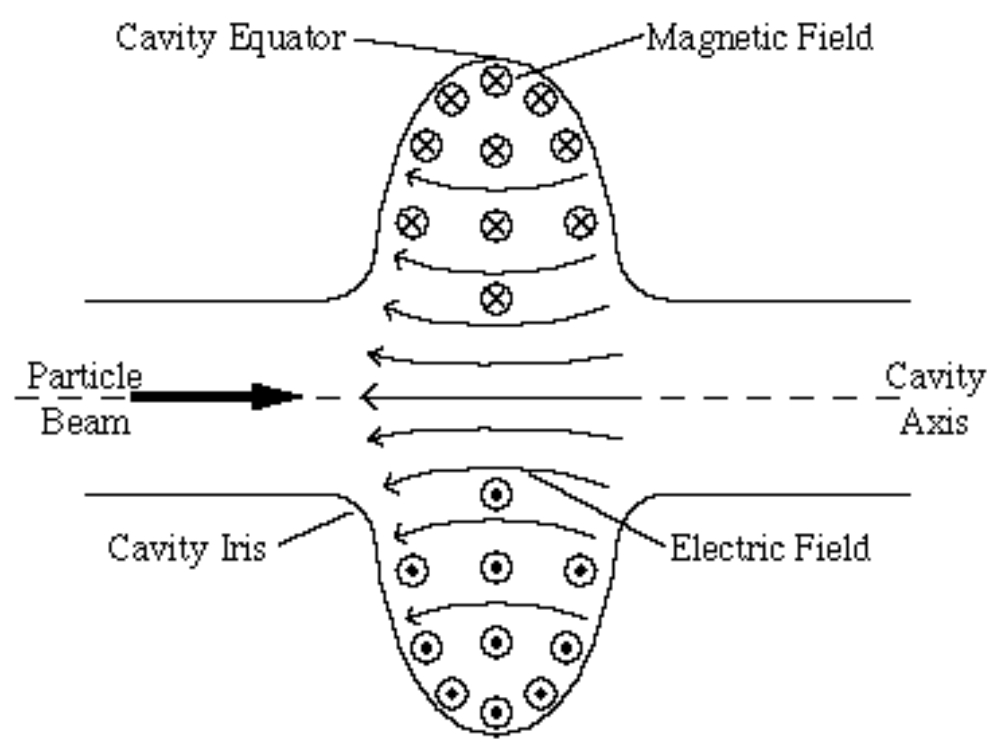

FIG. 2: The typical structure of a single-cell cavity resonating in $\mathrm{TM}_{010}$ mode where the electric field is parallel to the beam and maximum on the axis while the magnetic field is perpendicular to beam particle and is maximum at the equator.

to control the properties of this narrow layer in order to minimize the residual resistance of the cavity. The main goal of this work is to understand and evaluate the mechanisms and amount of dissipation caused by contribution of grain boundaries in polycrystalline materials using numerical approximation techniques to simulate the dynamics of dissipative vortices.

\subsection{GRAIN BOUNDARIES AS JOSEPHSON JUNCTIONS}

Grain Boundaries (GBs) are very common planar defects which arise naturally when a crystalline material forms as consequence of there being many nuclei that can initiate the process of crystal formation. GBs occur at the impinging interfaces between growing grains and they are usually classified according to the displacement and the rotation of the abutting crystals. For example, a $7^{\circ}[001]$-tilt boundary shown in Fig. 3a, connects two single crystals rotated with respect to each other by $7^{\circ}$ around the [001] direction which is common to both crystals and lies in the grain boundary plane. The misorientation is accommodated by formation of a series of edge dislocations along the low-angle grain boundaries. The distance $D$ between the dislocations is given by Frank's formula

$$
D=\frac{|\vec{b}|}{2 \sin (\alpha / 2)}
$$


where $|\vec{b}|$ is the magnitude of the Burgers vector $\vec{b}$ and $\alpha$ is the misorientation angle as sketched in Fig. 3b. A Burger vector is defined as the difference between the contours connecting corresponding lattice points in disturbed and undisturbed crystal around the dislocation. The formation of dislocations in grain boundaries is a consequence of topology and occur in common way for different materials, however, the microstructure is material dependent and strongly differs between ceramics and metals. As the misorientation angle increases, the dislocations are spaced closer together until they merge into a closed interface layer. Such high angle grain boundaries has more complicated structures for which the equation above no longer applies[2, 11].

(a)

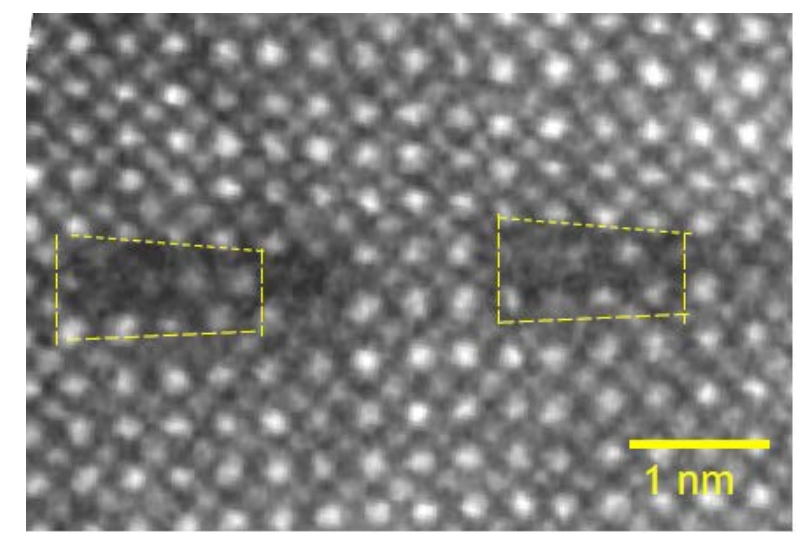

(b)

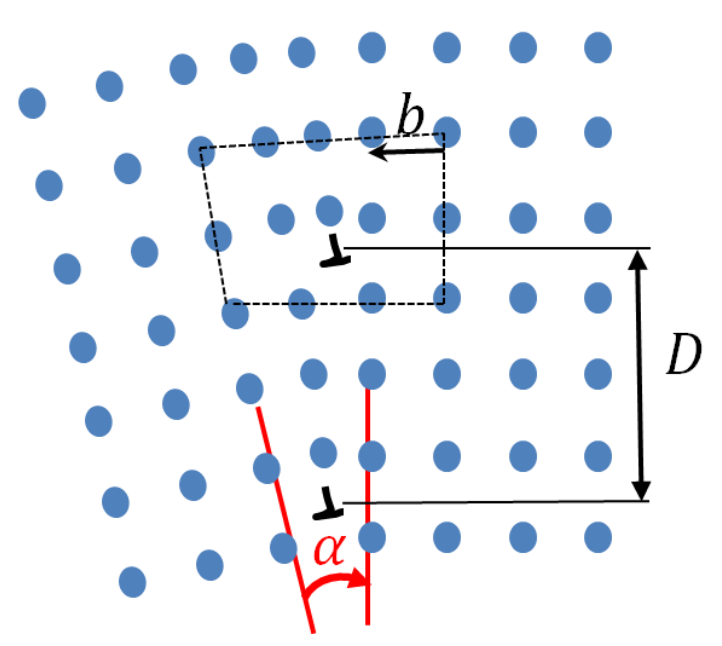

FIG. 3: (a) electron microscopy of dislocation cores in a $7^{\circ}[001]$-tilt grain boundary YBCO (taken from Ref.[1]) (b) Sketched diagram of a grain boundary formed along a continuous chain of dislocations in crystal lattice.

Grain boundaries are structural defects which by definition means that they affect most of the properties of the material. In superconductors a GB suppresses superconductivity locally, but the extent of suppression strongly depends on both the GB structure as well as the superconducting material. The length scale of the structural disorder, Thomas-Fermi screening length and the superconducting coherence length are the main parameters that can determine the superconducting properties of a GB[11].

In high- $\mathrm{T}_{c}$ superconductors, GBs even at low misorientation angles are weak superconducting links that impede the current flow giving rise to the electromagnetic granularity which is one of the serious obstacles for applications of the cuprates and the iron-based superconductors[11]. In planar GBs the critical current density across the GB, $J_{c, g b}(\alpha)$ falls off exponentially when the misorientation angle $\alpha$ exceeds a critical angle $\alpha_{c}$, where $\alpha_{c}$ can 
be as little as 3-5 $5^{\circ}$ for planar GBs of YBCO. The sinusoidal current-phase relation, magnetic field dependence of the critical current across the GB, appearance of Shapiro steps when the GB is irradiated and several other properties of typical Josephson junctions have been observed in different experiments verifying the weak-link behavior of GBs, specially in high$\mathrm{T}_{c}$ superconductors[2]. Figure 4 shows the current-voltage characteristic of a $24^{\circ}$ [001]-tilt boundary of $\mathrm{YBCO}$ at various temperatures[2].

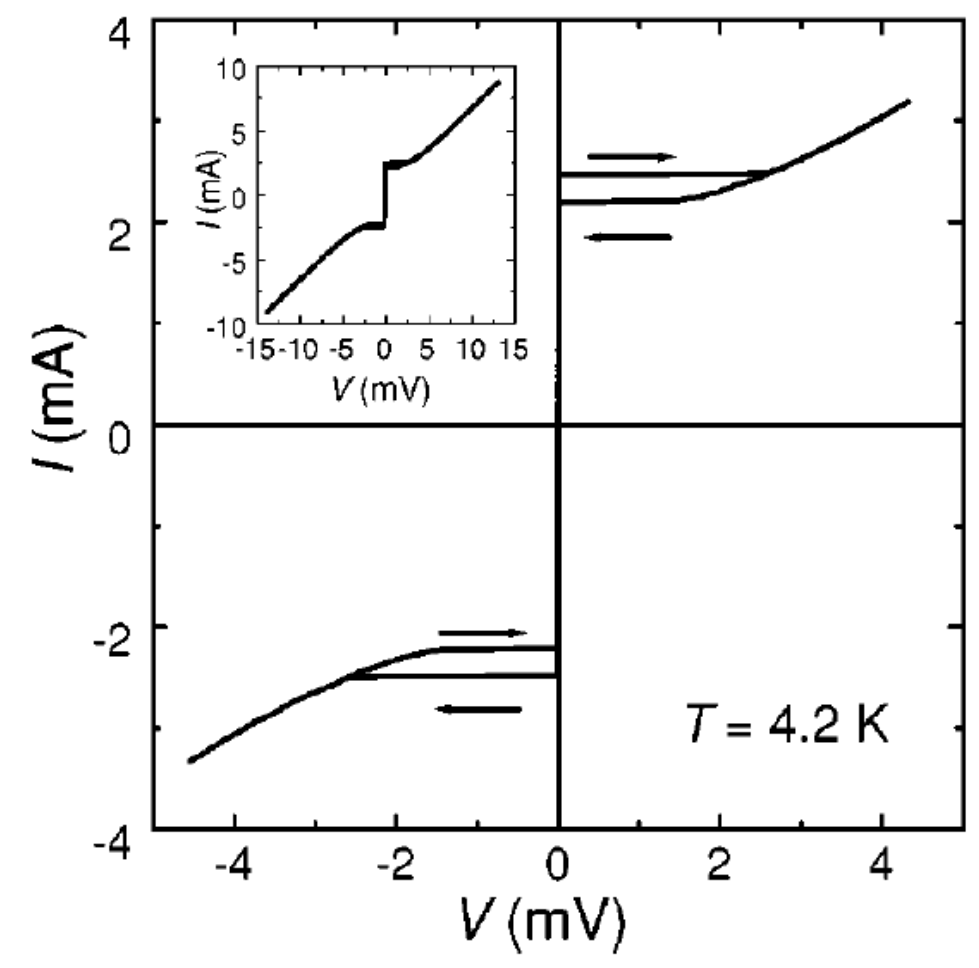

FIG. 4: Current-voltage characteristic of a $2.3-\mu \mathrm{m}$-wide bridge straddling a $24^{\circ}$ [001]-titl grain boundary in a 120-nm thick YBCO film. Figure is taken from Ref.[2].

SRF cavities are used to provide the maximum accelerating gradient, however, this depends on the maximum surface fields the cavity can sustain. Nevertheless, as mentioned before, the theoretical limit for the maximum surface magnetic field is called "superheating field", $H_{s}$, above which the surface barrier for penetration of Abrikosov vortices into the cavity disappears. Once vortices penetrate, they can flow and oscillate rapidly under the effect of screening currents resulting in an increase of surface resistance by several orders of magnitude which consequentially can cause thermal breakdown of the cavity. GBs in these polycrystalline SRF materials are also weak superconducting regions which can locally reduce the surface magnetic barrier due to their lower critical current density $J_{c, g b}$, so that vortices can penetrate at lower magnetic fields $H_{c, g b}<H_{s}$. In this case the dissipation occurs 
in a network of GBs on the surface of material which can significantly contribute to residual resistance or cause thermal instability at range of fields $H_{c, g b}<H<H_{s}$.

Generally in metallic superconducting materials like $\mathrm{Nb}$ and $\mathrm{Nb}_{3} \mathrm{Sn}$, two types of GBs can be considered: Low-angle GBs where the superconductivity is slightly suppressed, but the current transport can occur without significant decline of current density, hence a lowangle GB is also known as strong-linked or high- $J_{c}$ GB. Low-angle GBs allow strong pinning of vortices so that the current transport can occur without significant decline of current density. This property in metallic superconductors makes strong-linked GBs even favorable for current transport purposes as the higher the density of GBs, the higher is the critical current density. However, the second type of GBs occur when the misorientation angle is large and the GB turns into a weak-link superconducting region with current-blocking effects (low- $J_{c}$ GB). Weak-link grain boundaries in polycrystalline superconducting materials is known to provide easy channels for Josephson vortices to penetrate and oscillate under strong applications of current and magnetic field[38, 39]. Figures 5 and 6 show the typical structure of grain boundaries on surfaces of $\mathrm{Nb}$ and $\mathrm{Nb}_{3} \mathrm{Sn}$ films, respectively.

In high performance SRF cavities where the magnitude of rf screening currents at the inner surface of resonating cavity reaches almost the depairing current density $J_{d} \sim H_{c} / \lambda$, both types of GBs can be serious performance limiting factors. As later will be shown even slight reduction in current density across the GBs would facilitate penetration of Josephson and mixed Abrikosov-Josephson vortices which can produce significant losses due to various dissipative mechanisms and contribute to the residual surface resistance, as will be discussed later.

\subsection{JOSEPHSON EFFECT}

Early experimental demonstration of tunneling in superconducting/normal-conducting contacts by Giaever inspired Josephson[40, 41] to theoretically predict that Cooper pairs can tunnel through a thin insulating layer sandwiched between two superconducting bulks, giving rise to existence of a tunneling supercurrent even at zero voltage. Later shown to be correct for any weak link between two superconducting regions the term Josephson junction was coined for all such systems[42, 43]. Josephson prediction was soon verified experimentally by Anderson and Rowell[44].

In a rather simple treatment of a typical Josephson junction shown in Fig. 7, Feynman[45] was able to rederive Josephson equations by solving the Shrodinger equation written for a system of two wavefunctions using an additive coupling term between them. Provided that 


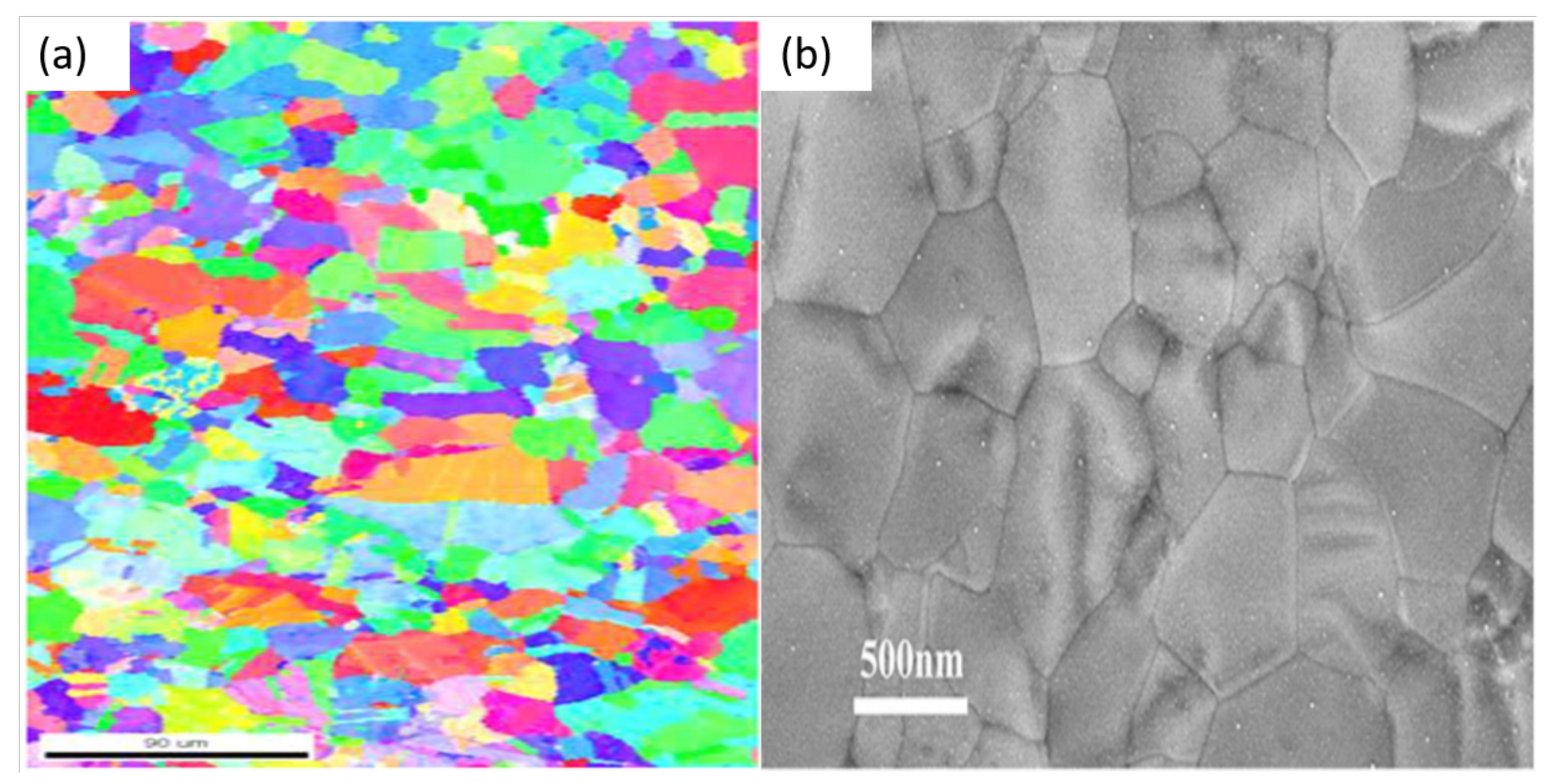

FIG. 5: (a) EBSD map showing the grain boudary structure for $\mathrm{Nb} / \mathrm{Cu}$ film and (b) SEM micrograph of the grain boudary structure in Nb film. Images taken from Ref.[3].

the gap between the two electrodes is small it can be shown that a flow of supercurrent given by $[42]$

$$
I_{s}=I_{c} \sin (\Delta \varphi)
$$

can exist due to the phase difference $\Delta \varphi=\varphi_{R}-\varphi_{L}$ between the two superconductors. This relation states the $d c$ Josephson effect. $I_{c}$ is called the junction critical current density and is the maximum supercurrent that can flow without resistance in the junction. $I_{c}$ is a characteristic property of junction which depends on junction geometry (thickness of the non-superconducting link, etc.) as well as temperature. In an attempt to calculate the temperature dependence of $I_{c}$, Ambegaokar and Baratoff[46] showed that the term $R_{n} I_{c}\left(R_{n}\right.$ is normal resistance of junction) is independent of junction geometry and only depends on material and temperature given by [5]

$$
R_{n} I_{c}=\frac{\pi \Delta(T)}{2 e} \tanh \left(\frac{\Delta(T)}{2 k_{B} T}\right) .
$$

In his second equation Josephson predicted that if a dc voltage is maintained across the superconducting electrodes of junction, the phase difference evolves with time according to

$$
\frac{d(\Delta \varphi)}{d t}=\frac{2 \pi}{\Phi_{0}} V .
$$




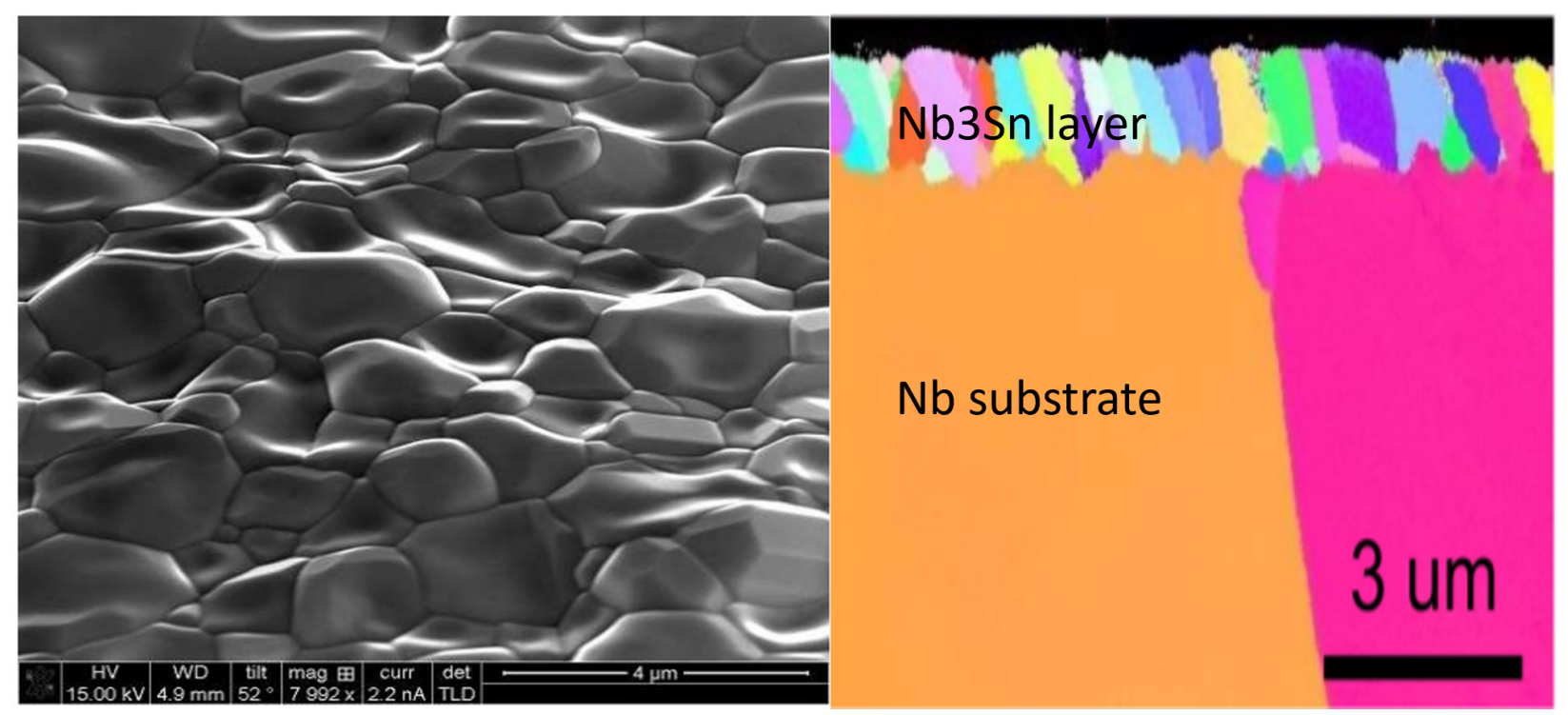

FIG. 6: Left: SEM image of the $\mathrm{Nb}_{3} \mathrm{Sn}$ surface. Right: Image of a cross section of $\mathrm{Nb}_{3} \mathrm{Sn}$ coating on $\mathrm{Nb}$ substrate. Images from Ref.[4].

which results in an alternating current with frequency $V / \Phi_{0}$ across the junction where $1 / \Phi_{0}=$ 483.6 MHz $/ \mu \mathrm{V}[42]$. The effect was initially verified experimentally by Yanson et al. [47] and Giaever[48] and is called the ac Josephson effect which is currently used worldwide as the most accurate way to define voltage standards. By integrating the electrical work $\int I_{s} V d t$ using (17) and (19), we will obtain the coupling free energy of the junction to be[5]

$$
F=\text { const. }-E_{J} \cos \Delta \varphi, \quad E_{J}=\frac{\Phi_{0} I_{c}}{2 \pi}
$$

which clearly indicates that the energy is minimum where the two phases are equal, $\Delta \varphi=0$.

\subsubsection{TYPES OF JOSEPHSON JUNCTIONS}

It was soon discovered that the Josephson effects which were initially predicted based on quantum mechanical tunneling of superelectrons through a thin layer of insulating barrier, are more general and occur whenever a weak link is connecting two strong superconducting electrodes. As shown in Fig. 8 the weak link can simply be a thin insulating layer as predicted originally (S-I-S), a normal metal for which superconductivity diffuses into normal contact through proximity effect $(\mathrm{S}-\mathrm{N}-\mathrm{S})$ or it can be the same superconducting material which is cut and become like a constriction or point contact (S-c-S). A more detailed treatment of different superconducting weak links is provided in the work of K. Likharev[49]. 


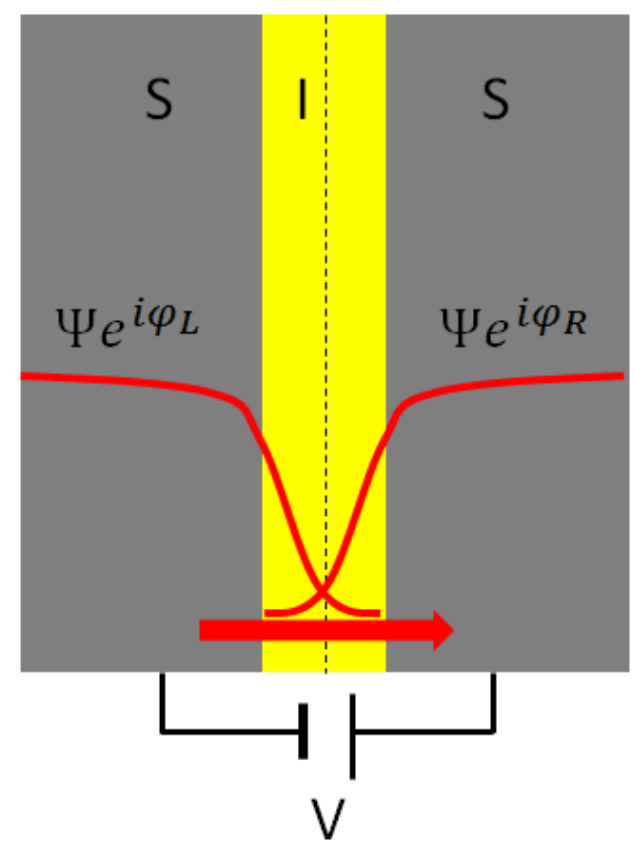

FIG. 7: A Josephson junction constructed by a thin layer of insulator (I) sandwiched between two superconducting electrodes $(\mathrm{S})$. Overlap of each electrode's superconducing wave function results in small supercurrent tunneling through the insulating barrier. A phase difference is maintained by applying the external voltage $(\mathrm{V})$.

\subsubsection{GAUGE-INVARIANT PHASE DYNAMICS OF SMALL JUNCTIONS}

A quick look at the Josephson current-phase relation in (17) unfolds that the well-defined physical quantity $I_{s}$ cannot be uniquely determined by $\Delta \varphi$ which is not a gauge-invariant quantity. To treat the problem we can replace $\Delta \varphi$ by the gauge-invariant phase difference $\theta$, defined by

$$
\theta=\Delta \varphi-\left(2 \pi / \Phi_{0}\right) \int \mathbf{A} \cdot d l
$$

where $\mathbf{A}$ is the vector potential, changing (17) to $I_{s}=I_{c} \sin \theta[5]$. Of course, so long as no magnetic field is present we can use $\theta$ and $\Delta \varphi$ interchangeably.

\section{RCSJ Model}

In absence of magnetic field a physical junction can be modeled by an ideal junction (shown by cross sign in Fig. 9) shunted by a resistance $R$ and capacitance $C$. For voltages below the gap $\left(V<V_{g}=2 \Delta / e\right)$ the resistance $R$ is of the order of normal resistance $R_{n}$ and is responsible for the energy dissipation due to flow of normal quasiparticles in finite voltage 


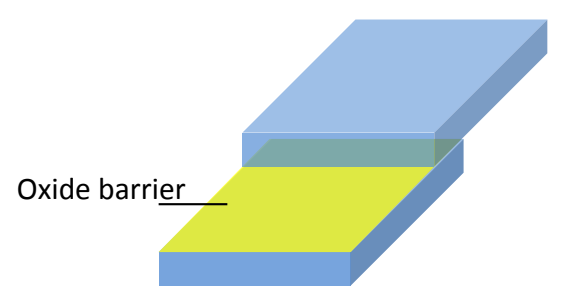

SIS

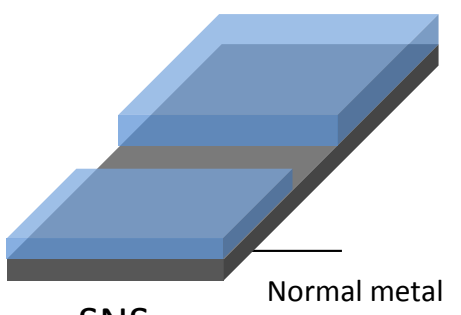

SNS

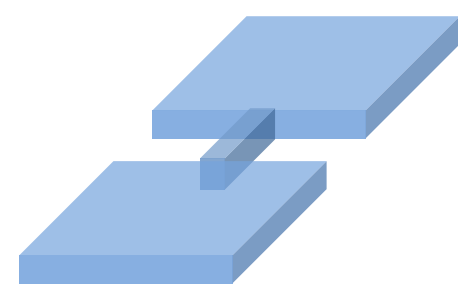

ScS

FIG. 8: Different types of Josephson junction can be constructed changing the weak-link barrier. Figure shows weak-links provided by an insulator (SIS), normal metal (SNS) or a constriction $(\mathrm{ScS})$.

regime. At very low temperatures the normal resistance $R_{n}$ rises exponentially as $R_{n} e^{\Delta / k T}$ due to freeze-out of quasi-particles at low temperatures. The capacitance $C$ is taken as the geometric shunting capacitance between the two superconducting electrodes in the junction.

Within the RCSJ model, the total current passing through the circuit sketched in Fig. 9 is the combination of supercurrent $I_{c} \sin \theta$, normal current $V / R$ and displacement current $C d V / d t$ due to time-varying electric field between the superconducting electrodes. By elimination of $V$ using (19) in favor of $\theta$, we obtain a second order nonlinear differential equation[5, 42, 43]

$$
I=I_{c} \sin \theta+\frac{\Phi_{0}}{2 \pi R} \frac{d \theta}{d t}+\frac{C \Phi_{0}}{2 \pi} \frac{d^{2} \theta}{d t^{2}} .
$$

which in dimensionless form becomes

$$
\frac{d^{2} \theta}{d \tau}+\eta \frac{d \theta}{d \tau}+\sin \theta=\beta
$$

where $\beta=I / I_{c}$ is dimensionless bias current density and differentiation was done with respect to dimensionless time $\tau=\omega_{J} t$, with

$$
\omega_{J}=\left(\frac{2 \pi I_{c}}{C \Phi_{0}}\right)^{1 / 2}
$$

being called the Josephson plasma frequency of the junction, and the damping factor $\eta$ is defined as

$$
\eta=\left(\omega_{J} R C\right)^{-1} .
$$

In the above equation of (23), $\eta=\beta_{c}^{-1 / 2}$ is the only device-dependent parameter, where $\beta_{c}$ was initially introduced as damping parameter by Stewart and McCumber[50, 51]. 


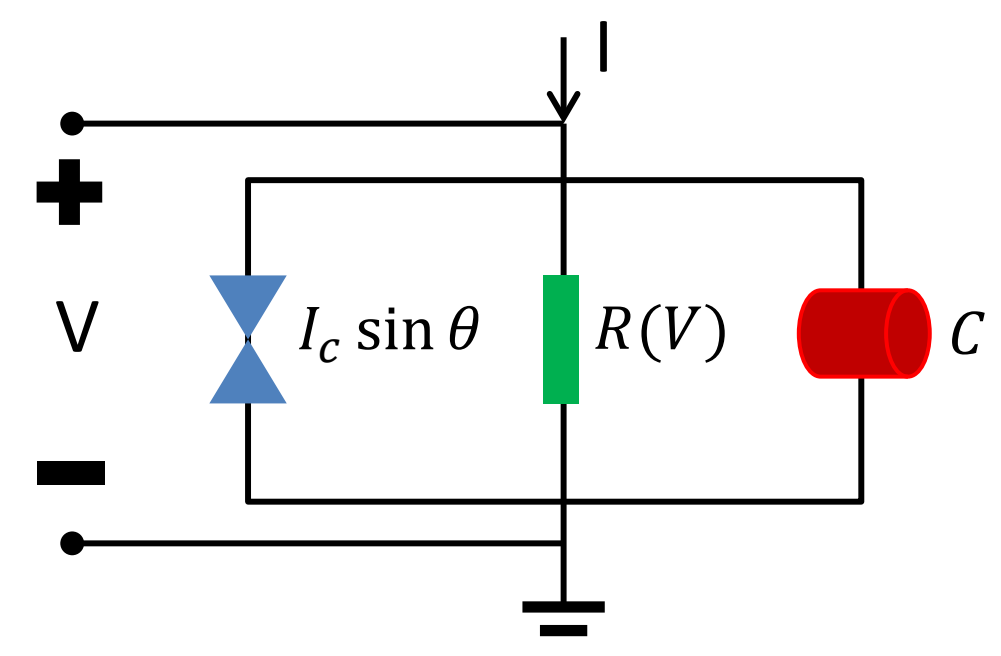

FIG. 9: A real small Josephson junction can be modeled as an equivalent RCSJ circuit where an ideal junction is shunted by a capacitor and resistor.

\section{Tilted-Washboard Model}

Different mechanical analogs have been put forth to qualitatively describe the dynamics of the junction and give insight to the problem before delving into numerical studies. Looking back to (22), it describes the equation of motion of a particle with mass $\left(\Phi_{0} / 2 \pi\right)^{2} C$ moving along the direction of $\theta$ in an effective potential given by [5]

$$
U(\theta)=-E_{J} \cos \theta-\left(\frac{\Phi_{0} I}{2 \pi}\right) \theta
$$

while dragged by a viscous force of $\left(\Phi_{0} / 2 \pi\right)^{2}(1 / R) d \theta / d t$. As sketched in Fig. 10, the shape of this potential is a tilted cosine with slope proportional to bias current $I$, resembling a tilted washboard hence the name. To have a physical intuition we consider a mass particle moving in a gravitational field along the track of (26). So long as $I<I_{c}$ the particle will eventually be stabilized at the bottom of a local minima which indicates that a static solution is allowed and the voltage across remains zero. Furthermore, at $I=I_{c}$ the local minima of tilted cosine become horizontal in a continually downward slope, therefore no stable equilibrium solution will exist for $I>I_{c}$ and the particle would enter a running state where the voltage is no longer zero. Detailed dynamics of the particle which depends on bias current, damping in the junction as well as thermal fluctuations will be considered later.

\subsubsection{I - V CHARACTERISTICS AT $\mathrm{T}=0$}




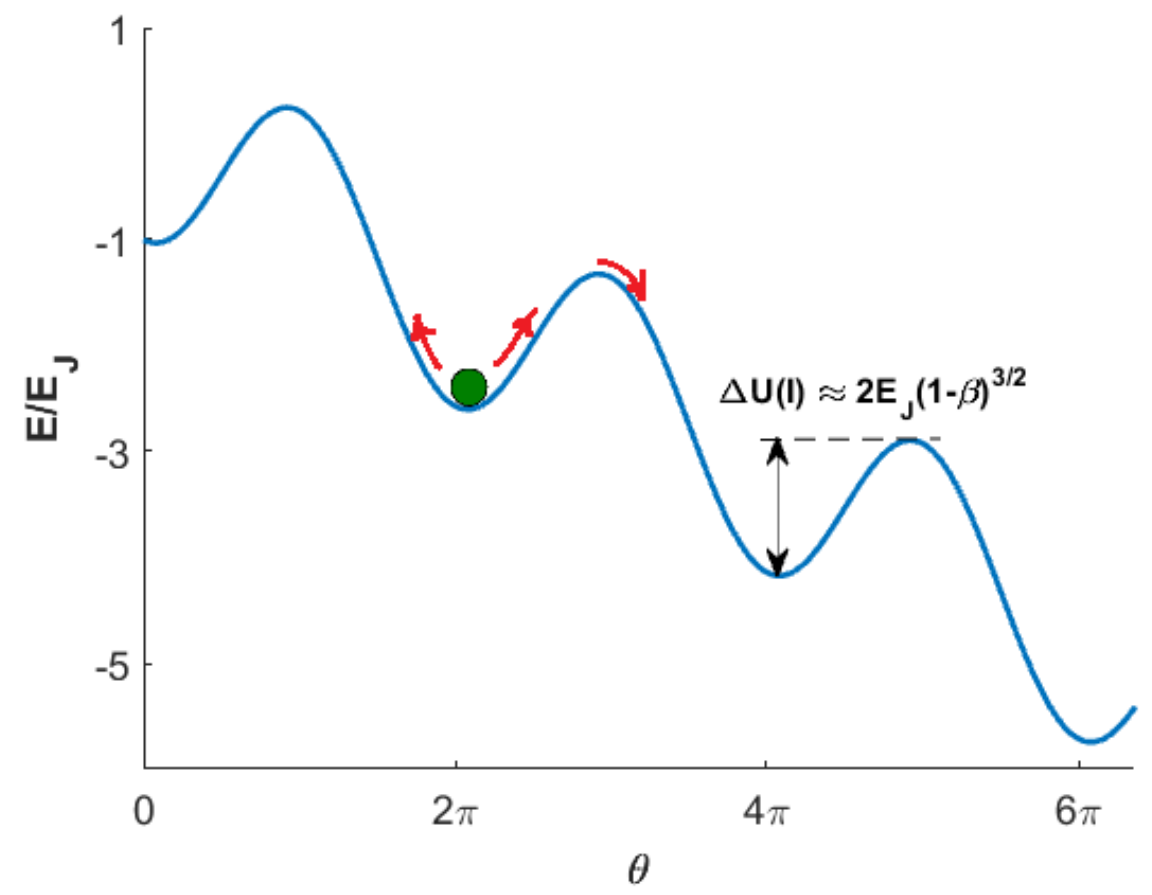

FIG. 10: A tilted washboard potential can analogously model the motion of guage-invariant Josephson phase as a damped particle. The sinusoidal potential is tilted due to application of bias curretnt.

As pointed out in previous section, a static solution of $\theta=\sin ^{-1} \beta$ is allowed for (23), provided that $\beta<1$. For values of $\beta>1$, all solutions are time-dependent and they determine the dc $I-V$ curve for the junction. Here we discuss the solutions of (23) in absence of thermal fluctuations $(T=0)$ and in different damping regimes:

\section{Overdamped Junction}

In a highly damped junction where $C$ is small (i.e. $\eta \gg 1$ ), the first term in $(23)$ can be neglected and the remaining equation is solved analytically which results in a time-averaged dc voltage given by[5]

$$
V=I_{c} R\left(\beta^{2}-1\right)^{1 / 2}
$$

As shown in Fig. 11a, the curve asymptotically converges to Ohm's law $V=I R$ for $I \gg I_{c}$. The dc voltage is time average of a series of pulses with maximum amplitude of $I_{c} R$ wherein each pulse the phase advances by $2 \pi$ periodically. This regime in tilted-washboard model corresponds to a low mass particle moving under a viscous drag force which dominates the inertial effects. 


\section{Underdamped Junction}

When capacitance is large enough so that $\eta<1$, the junction behavior becomes hysteretic. As plotted in Fig. 11b, once current exceeds $I_{c}$, a finite voltage appears jumpwise across the junction where the phase increases non-stop with the rate of $2 \pi V / \Phi_{0}$. Reducing the current back, the voltage does not fall to zero even at currents well below the $I_{c}$. In analogy with the washboard model, this corresponds to the case where the inertia of the particle is large enough that carries it up the barriers and the damping is not high enough to stop it momentarily. At $T=0$ and state of no damping, retrapping will not happen until the bias current becomes zero again.

(a)

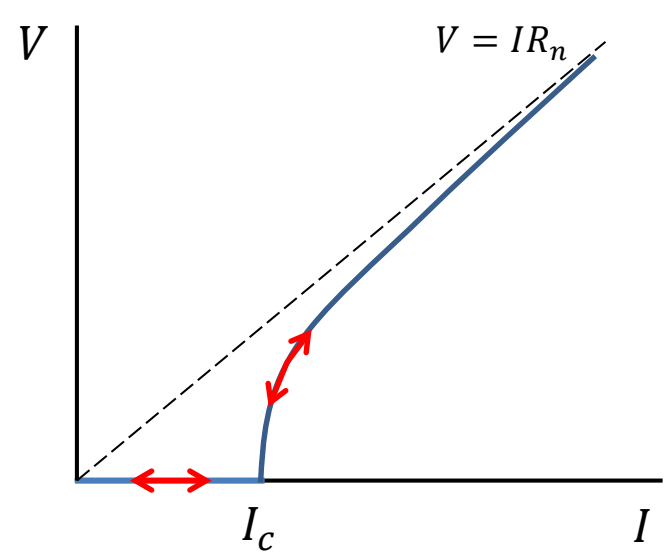

(b)

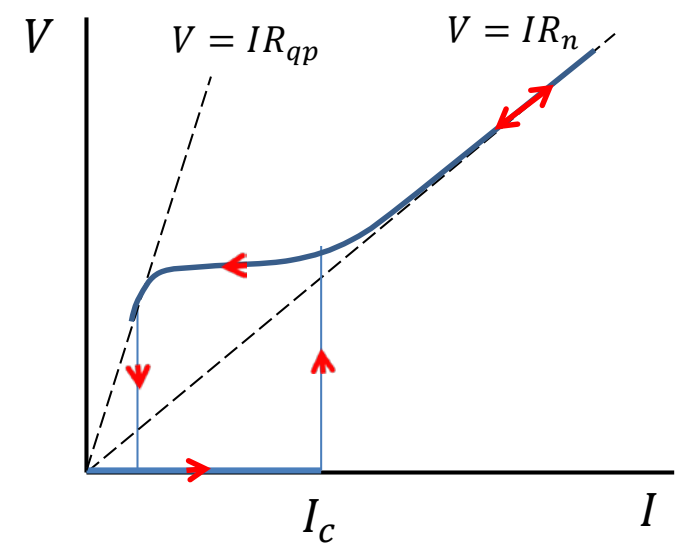

FIG. 11: Current-voltage characteristics of an overdamped (a) and underdamped (b) junciton. The hysteretic effects in underdamped junctions result in jumpwise transitions in $I-V$ curves.

\subsubsection{THERMAL FLUCTUATION EFFECTS}

The I-V characteristic of a junction can be strongly modified when $T>0$, in other words, thermally activated processes strongly affect the dynamics of the junction. Following the relation in (18), for $T \ll T_{c}$ the ratio

$$
\frac{2 E_{J}}{k_{B} T}=1.76\left(\frac{R_{Q}}{R_{n}}\right)\left(\frac{T_{c}}{T}\right)
$$

exists where the quantum resistance $R_{Q}=h / 4 e^{2}=6,453 \Omega[5]$. The ratio tells that the thermal fluctuation effects are unimportant in both cases of low temperatures $\left(T \ll T_{c}\right)$ or when $R_{n} \ll R_{Q}$, because the barrier energy is much larger than the typical energy of thermal fluctuations, $k_{B} T$. 


\section{Underdamped Junctions}

In an underdamped junction, thermally activated escapes from one minima over the barrier to the next can happen with the probability of $\omega_{J} e^{-\Delta U(I) / k_{B} T}$ per unit time where $\Delta U(I)$ is the current-dependent barrier height shown in Fig. 10 and is given approximately by

$$
\Delta U(I) \approx 2 E_{J}\left(1-I / I_{c}\right)^{3 / 2}
$$

which tends to zero as $I \rightarrow I_{c}$, as expected[5]. Therefore, in an upward sweep of bias current this probability increases until at some "switching current" $I_{s w}$ below $I_{c}$ the particle escapes from its minima and since negligible damping, it accelerates down the potential reaching a terminal velocity and never retraps in another minima again. This state corresponds to an immature switching of the junction to non-zero voltage state which happens on a distribution of currents below the critical current $I_{c}$. Furthermore, thermal fluctuations surprisingly increase the retrapping current once the bias current is swept downward from a non-zero voltage state. Since both escpaing and retrapping are stochastic phenomena, they happen over a distribution of currents as shown in Fig. $12 \mathrm{~b}$.

\section{Overdamped Junctions}

Thermal fluctuations also modify the I-V characteristic of a highly damped junction through thermally activated phase slips which cause a finite nonlinear resistance to appear even below $I_{c}$. In these phenomena the phase point "diffuses" over the barriers in a continuous manner because high damping brings back the phase point into equilibrium before diffusing into another minima again. This process is strikingly different from escaping which happens in a lightly damped junction where the phase never retraps in another minima once it escapes the barrier. Figure 12a shows how I-V curve changes with the increase of temperature.

\subsubsection{SHAPIRO STEPS}

The ac Josephson effect can be further realized by adding an alternating voltage source of $V_{1} \cos \omega_{1} t$ to the already applied dc voltage $V_{0}$. In an experimental observation, Shapiro[52] spotted stepping structure in the dc $I-V$ curve where each step in the supercurrent response takes place at voltages $V_{n}=\left(n \Phi_{0} / 2 \pi\right) \omega_{1}$. For a junction biased by voltage $V=V_{0}+V_{1} \cos \omega_{1} t$, it can be shown that the phase difference across the junction becomes

$$
\theta(t)=\theta_{0}+\omega_{0} t+\left(2 \pi V_{1} / \Phi_{0} \omega_{1}\right) \sin \omega_{1} t
$$


(a)

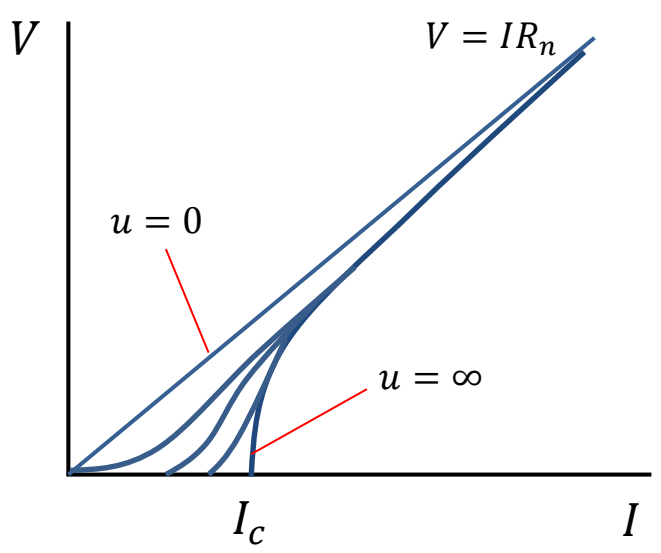

(b)

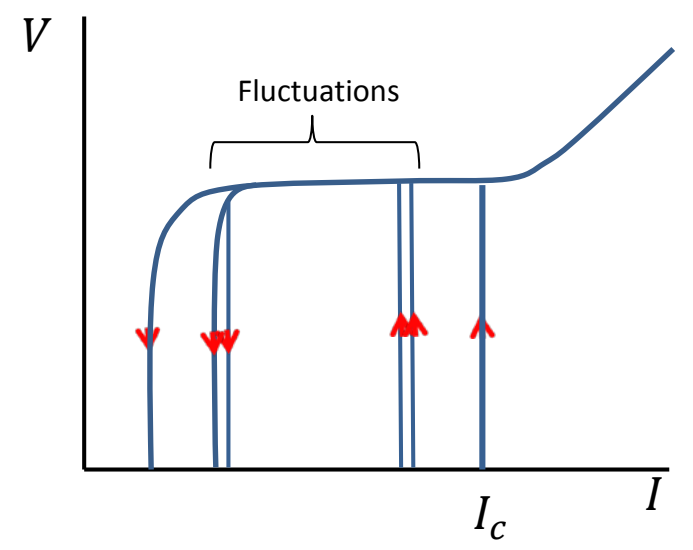

FIG. 12: Thermal fluctuations result in smearing of the $I-V$ curve in overdamped junction (a), while it reduces the hysteresis in an underdamped junction (b). Here $u=\hbar I_{c} / e k_{B} T$.

where $\theta_{0}$ is constant of integration and $\omega_{0}=2 \pi V_{0} / \Phi_{0}$. Using (17), standard mathematical expressions and Bessel functions $J_{n}(x)$, we will have[5]

$$
I_{s}=I_{c} \sum(-1)^{n} J_{n}\left(2 \pi V_{1} / \Phi_{0} \omega_{1}\right) \sin \left(\theta_{0}+\omega_{0} t-n \omega_{1} t\right)
$$

which contributes dc components to supercurrent whenever $\omega_{0}=n \omega_{1}$ (Fig. 13). The width of each dc current is a function of Bessel function properties. If junction is biased with ac current, a similar effect happens but the solution is more complicated and must be treated numerically.

\subsubsection{MAGNETIC FIELD EFFECTS}

To consider the effect of magnetic field on phase dynamics of junction, we start by G$\mathrm{L}$ second equation for current density in each superconducting electrodes of the junction sketched in Fig. 14. The gauge-invariant phase difference in each electrode can be written as

$$
\nabla \theta=\frac{2 \pi}{\Phi_{0}}\left(\mu_{0} \lambda^{2} \mathbf{J}_{\mathbf{s}}+\mathbf{A}\right)
$$

where $\mathbf{A}$ is the vector potential related to applied magnetic field by $\nabla \times \mathbf{A}=\mu_{0} \mathbf{H}[5,42]$. Integration of (32) along the contours of $C_{R}$ and $C_{L}$ deep into superconductors where $\mathbf{J}_{\mathbf{s}}=\mathbf{0}$ and neglecting the difference of magnetic field across the thickness of the junction yields

$$
\theta(x+d x)-\theta(x)=\frac{2 \pi}{\Phi_{0}} \oint \mathbf{A} \cdot d l
$$



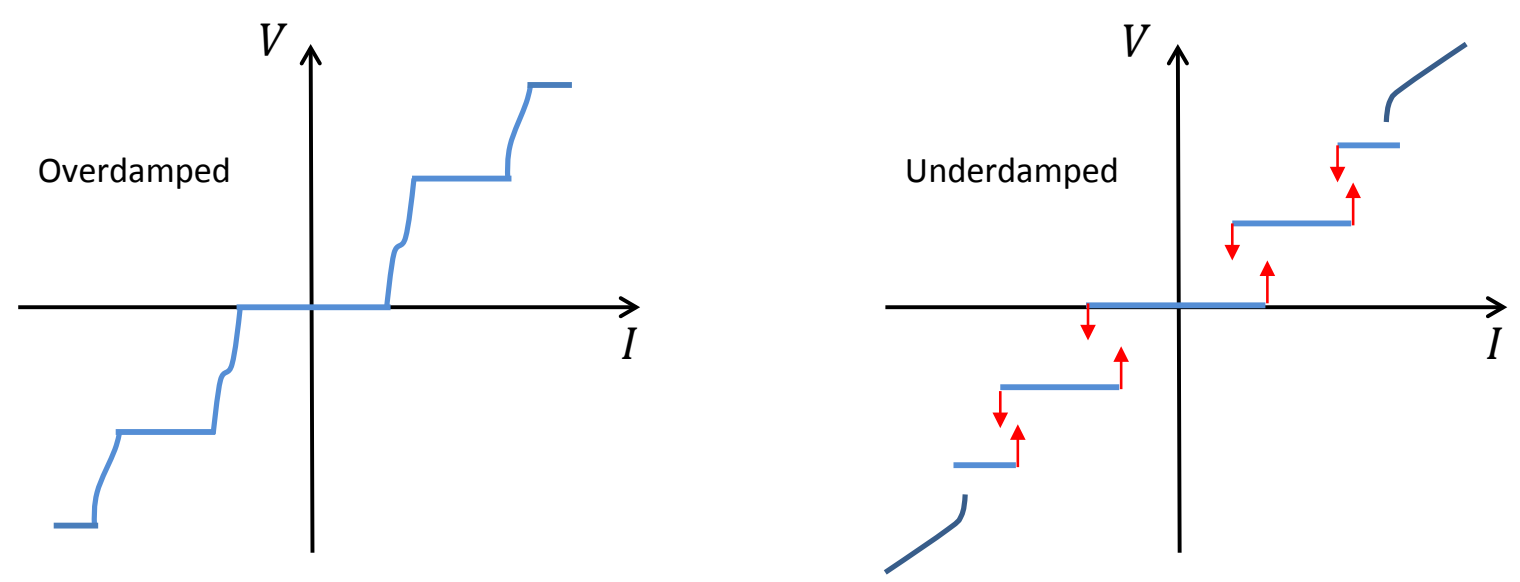

FIG. 13: Shapiro current-voltage steps in $I-V$ curves of overdamped (a) and underdamped (b) junctions.

where assuming that magnetic field is almost uniform along the spatial interval of $d x$, the line integral in (33) is the magnetic flux enclosed by the contours and can be replaced by

$$
\oint \mathbf{A} \cdot d l=\mu_{0} H\left(2 \lambda+d_{j}\right) d x
$$

where $d_{j}$ is the thickness of the junction. Therefore, the spatial variation of phase along the junction can be given by $[5,42]$

$$
\frac{d \theta}{d x}=\frac{2 \pi \mu_{0}}{\Phi_{0}}\left(2 \lambda+d_{j}\right) H
$$

\section{Small Junctions, Negligible Screening}

In a small junction where tunneling currents are small and their self-field is negligible, the magnetic filed can penetrate into junction without variation. Integration of (35) and using (17) to find the tunneling current, we obtain[5, 42]

$$
\begin{gathered}
\theta(x)=\frac{2 \pi \mu_{0} d}{\Phi_{0}} H x+\theta_{0} \\
J(x)=J_{c} \sin \left(\frac{2 \pi \mu_{0} d}{\Phi_{0}} H x+\theta_{0}\right)
\end{gathered}
$$

where $d=2 \lambda+d_{j}$. Here (36) shows a linear spatial variation of phase with slope proportional to field and therefore (37) exhibits a periodic distribution of current inside the junction. It can 


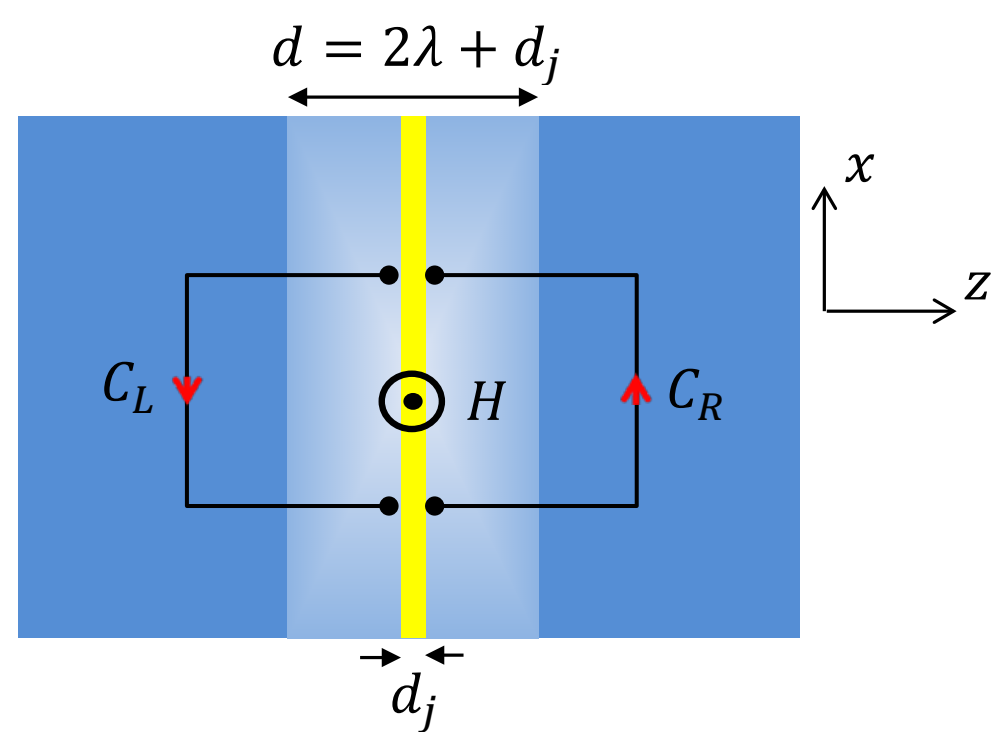

FIG. 14: Diagram of an extended junction (yellow). Spatial variations of phase difference along the juntion due to applied magnetic field can be calculated using the contours $C_{L}$ and $C_{R}$. The light colored region shows the extent of magneitc field penetration.

be shown that the maximum supercurrent across the small junction reduces with magnetic field given by $[5,42]$

$$
I_{c}(H) / I_{c}(0)=\left|\frac{\sin \left(\pi \Phi / \Phi_{0}\right)}{\left(\pi \Phi / \Phi_{0}\right)}\right|
$$

which represents a Fraunhofer pattern as illustrated in Fig. 15. Here $\Phi$ is the amount of flux present inside the junction. The relation (38) becomes more complicated if the critical current is non-uniform or the junction area is not rectangular.

\subsection{PHYSICS OF LONG JOSEPHSON JUNCTIONS (LJJ)}

In a large junction however (Fig. 16), when Josephson tunneling currents are strong enough so their self-field is no longer negligible; a magnetic field is screened out of the junction over the Josephson penetration depth $\lambda_{J}=\left(\Phi_{0} / 2 \pi \mu_{0} d J_{c}\right)^{1 / 2}$, from the edges of the junction $[5,42,43]$. This means that in a large junction the distribution of tunneling currents along the junction is non-uniform and confined mostly around the edges, even at zero magnetic filed. A large junction similar to a type-II superconductor screens out magnetic field up to some critical field above which the field penetrates inside the junction in form of solitonic vortices which will be shown later.

Since magnetic field is no longer constant we use Maxwell equation for the field inside 


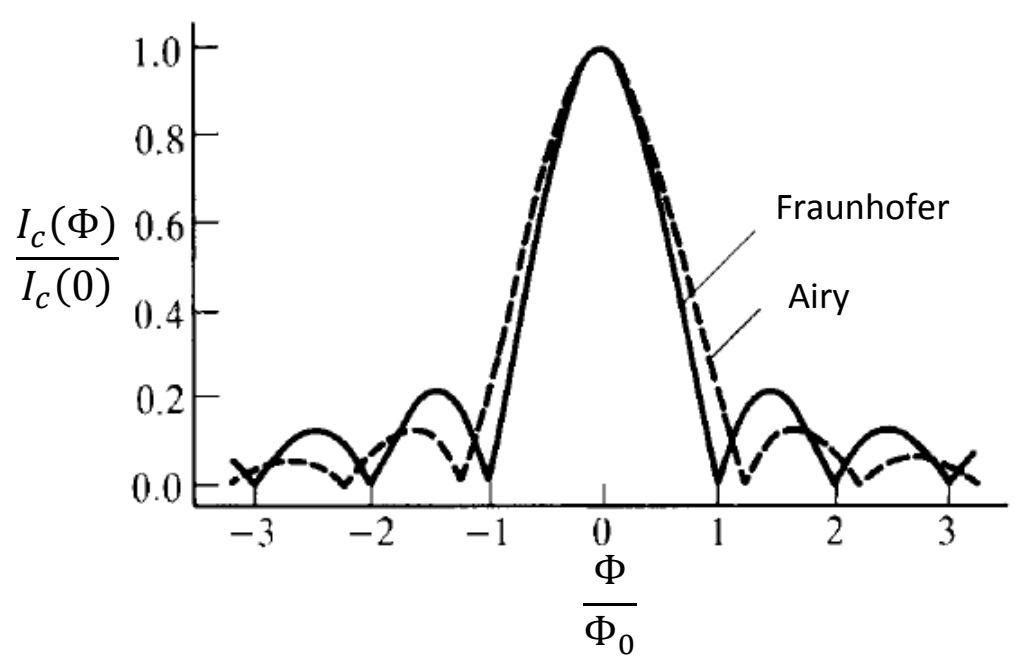

FIG. 15: Dependence of the junction maximum supercurrent on enclosed flux. Fraunhofer and Airy diffraction patterns for rectangular and circular junctions, respectively. Figure is taken from Ref.[5].

the junction $d H / d x=J$ along with (17) and (35) to write

$$
\frac{d^{2} \theta}{d x^{2}}=\frac{1}{\lambda_{J}^{2}} \sin \theta, \quad \lambda_{J}=\left(\frac{\Phi_{0}}{2 \pi \mu_{0} d J_{c}}\right)^{1 / 2} .
$$

Equation (39) is called the static sine-Gordon equation [5, 42, 43] and has the same form as one for the pendulum, so insight to the solutions can be gained by analogy. For small oscillations of phase, $\theta \ll 1$, phase is decreasing exponentially in the form $\theta \sim e^{ \pm x / \lambda_{J}}$ from the edges, describing the case for the complete magnetic field screening. If the field is strong enough so the current at the edges of the junction would reach $J_{c}$, magnetic field would penetrate into junction in form of Josephson vortices. In pendulum analogy the onset of Josephson vortex penetration corresponds to the situation when pendulum is energetic enough to go over the top. The strongest magnetic field that is screened from the interior of the junction and above which vortices can penetrate is given by $[5,42,43]$

$$
H_{1}=2 \lambda_{J} J_{c} \simeq H_{c}\left(\frac{J_{c}}{\kappa J_{d}}\right)^{1 / 2}
$$

and plays the role of a superheating field at which the edge energy barrier for the penetration of Josephson vortices disappears. However, for the field region $H_{c 1 J}<H<H_{1}$ where $H_{c 1 J}=(2 / \pi) H_{1}$, the solution is only metastable and the screening is no longer exponential near the edge but becomes so in the interior. Therefore $H_{c 1 J}$ is the maximum field above 
which the screening solutions are thermodynamically unstable. The nature of Josephson vortices is the subject of next chapter and will be discussed in more detail.

When one of the dimensions of a Josephson junction is much larger than the others, it is called a Long Josephson Junction (LJJ). In particular, $L \gg \lambda_{J} \gg W$ where $L$ and $W$ are the length and width of the junction respectively, as sketched in Fig. 16a and $\lambda_{J}$ is the Josephson penetration length over which the field is screened inside the junction. As noted before, in a large junction with screening, the phase difference $\theta(x, t)$ also varies over $\lambda_{J}$. The assumption of $W \ll \lambda_{J}$ is made in order to investigate the electrodynamics of LJJ only in one dimension. A LJJ can be fabricated in different geometries depending on the subject of interest. Some of the more common types of LJJs have been shown in Fig. 16.

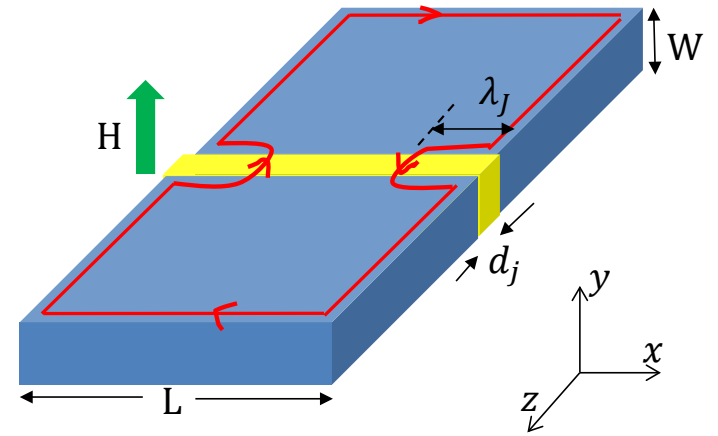

(a) Edge junction (Grain boundary)

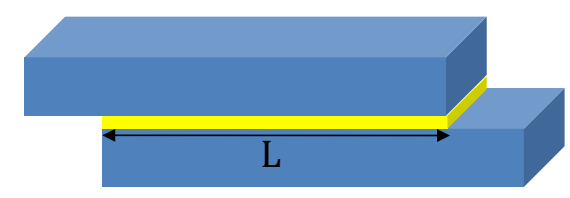

(c) Inline junction

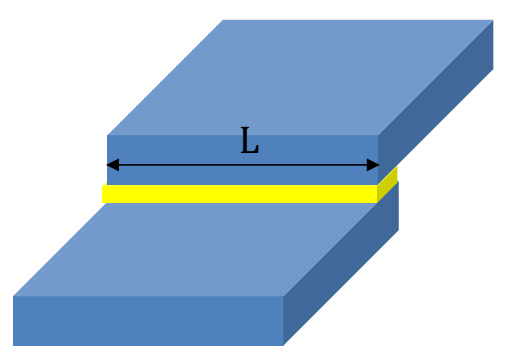

(b) Overlap junction

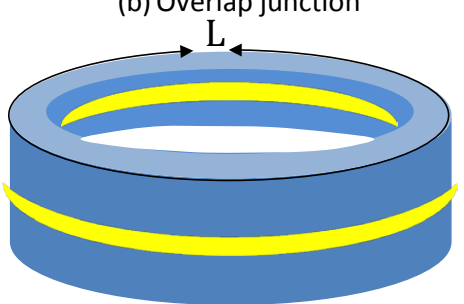

(d) Annular junction

FIG. 16: Different long Josephson junction geometries.

\subsection{ELECTRODYNAMICS OF LJJ}

Several models and methods[42, 43] can be used in order to describe the behavior of a single LJJ. While the main equation is the same, different terms might be added or removed associated with the specific features of the problem. Here we resort to the probably more simpler method which considers the main features of a single LJJ behavior.

Consider the long junction pictured in Fig. 16a which consists of identical superconductors containing a nonsuperconducting barrier with thickness $d_{j}$, permittivity $\epsilon_{0} \epsilon_{r}$ and 
resistivity $\rho$. In presence of magnetic field applied in $y$ direction, flux can penetrate into superconducting electrodes over the length $\lambda$ while the electric field is negligible everywhere except in the barrier. To set up the equations for variation of electric field $E(x, t)$ and magnetic field $H(x, t)$ inside the junction, we start off by Maxwell equation for Ampere's law

$$
\frac{\partial}{\partial x} H(x, t)=J_{z}(x, t)+\epsilon_{0} \epsilon_{r} \frac{\partial}{\partial t} E(x, t)
$$

where the junction area is taken in $x-y$ plane and the tunneling currents flow in $z$ direction. The total current can be written similar to RCSJ model

$$
J_{z}(x, t)=J_{c} \sin \theta(x, t)+\frac{1}{\rho d_{j}} E(x, t)+J_{e x t}
$$

as combination of Josephson, quasiparticle and transport currents. Using the relation between magnetic field and phase difference in (35), one can express (41) as wave equation for $\theta[42,43]$ :

$$
\frac{1}{\omega_{J}^{2}} \frac{\partial^{2}}{\partial t^{2}} \theta(x, t)+\frac{1}{\omega_{c}} \frac{\partial}{\partial t} \theta(x, t)=\frac{1}{\lambda_{J}^{2}} \frac{\partial^{2}}{\partial x^{2}} \theta(x, t)-\sin \theta(x, t)+\beta
$$

where we introduced $\omega_{J}=\left(2 \pi J_{c} / \Phi_{0} C\right)^{1 / 2}$ as Josephson plasma frequency, $\omega_{c}=2 \pi R_{j} J_{c} / \Phi_{0}$ as characteristic frequency, $R_{j}=\rho d_{j}$ as quasiparticle specific resistance per unit area and specific capacitance $C=\epsilon_{0} \epsilon_{r} / d_{j}$ per unit area of junction. In dimensionless form, equation (43) takes the form of[43]

$$
\ddot{\theta}+\eta \dot{\theta}=\theta^{\prime \prime}-\sin \theta+\beta
$$

where the overdot and prime denote partial derivatives with respect to the dimensionless time $\omega_{J} t$ and coordinate $x / \lambda_{J}$ while $\eta=1 / \omega_{J} R_{j} C$ is known as damping factor, as defined before. This equation is known as perturbed sine-Gordon equation and has been one of the most widely used equations to describe topological defects in charge and spin density waves[53], commensurate-incommensurate transitions [54, 55], magnetic domain walls[56], dislocations in crystals $[57,58]$, kinks on DNA molecules[59, 60] and finally Josephson junctions.

\subsection{SOLUTIONS OF SINE-GORDON (SG) EQUATION}

In general equation (44) does not have a known analytical solution and must be solved numerically. Nevertheless, this equation in conjunction with proper boundary conditions specified by the problem geometry can describe the electrodynamics of a homogeneous LJJ with good enough accuracy and a wide range of physical quantities can be obtained from the dynamic phase solutions. 
Exact treatment of boundary conditions is very problem dependent, however there are some general rules that have to be considered. In linear junctions magnetic field can be applied both uniformly through the transport current $\beta$ term or non-uniformly using the phase-field relation in (35) applied to the junction ends. In annular junctions the boundary conditions are so that the single-valuedness of the wave function is justified throughout the junction (phase is periodic with respect to the length of the junction).

In an infinitely long linear junction there exists analytic solutions to the unperturbed sine-Gordon equation

$$
\ddot{\theta}=\theta^{\prime \prime}-\sin \theta
$$

which can be classified in three main types[42].

- Plasma oscillations. These are small amplitude plain waves in the form of $\theta_{a} e^{i\left(k x-\omega_{k} t\right)}\left(\left|\theta_{a}\right| \ll 1\right)$ that can propagate along the junction. The dispersion relation between frequency $\omega_{k}$ and wave vector $k$ is given by

$$
\omega_{k}(k)=\omega_{J} \sqrt{1+\lambda_{J}^{2} k^{2}}
$$

from which we can determine the phase velocity of an electromagnetic wave in a LJJ as

$$
v_{p h}=\frac{\omega_{k}}{k}=c_{s} \sqrt{1+\frac{1}{k^{2} \lambda_{J}^{2}}}
$$

while the group velocity is given by

$$
v_{g}=c_{s} \frac{\lambda_{J} k}{\sqrt{1+\lambda_{J}^{2} k^{2}}} .
$$

where $c_{s}=\omega_{J} \lambda_{J}=\left(d_{j} / \epsilon_{r} d\right)^{1 / 2} c$ is known as Swihart velocity[61], typically a few percent of light velocity. As plotted in Fig. 17, it clearly shows that $v_{g} \leq c_{s} \leq v_{p h}$.

- Solitons. The most famous solution of (45) is given by

$$
\theta(x, t)=4 \tan ^{-1}\left(\exp \left[ \pm \frac{x-\left(v / c_{s}\right) t-x_{0}}{\sqrt{1-\left(v / c_{s}\right)^{2}}}\right]\right)
$$

which describes a moving kink (soliton) that changes $\theta$ from 0 to $2 \pi$ from $-\infty$ to $+\infty$ for the $(+)$ sign or vice versa, an antikink (antisoliton) for the $(-)$ sign. This solution describes a Josephson vortex which consists of localized current within the distance $2 \lambda_{J}$ which changes direction at the center of moving vortex $x_{0}+\left(v / c_{s}\right) t$. Equation (45) is Lorentz-invariant where $c_{s}$ plays the role of the light velocity. Therefore, solitons 


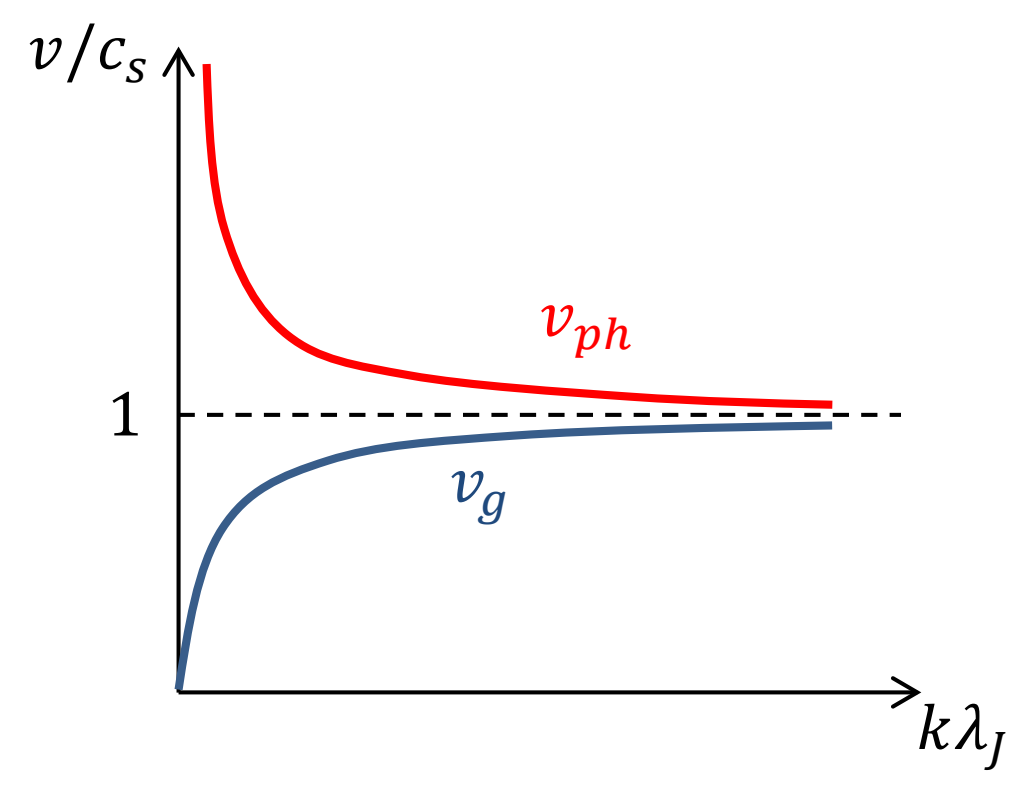

FIG. 17: Phase and group velocity curves of electromagnetic waves propagation obtained from dispersion relation of a long Josephson junction described by (45).

will act like relativistic particles which are subjected to Lorentz contraction, hence the size of a soliton moving with constant velocity $v$ will shrink to $\left(1-v^{2} / c_{s}^{2}\right)^{1 / 2} \lambda_{J}$ from its static size $\lambda_{J}$. Figure 18 shows profiles of Josephson vortex solutions in phase, field and current representations both in static and dynamic states. In spite of contraction, a Josephson vortex always carries a quantum of magnetic flux, hence also called fluxon.

- Breathers. Similar to Abrikosov vortices, Josephson fluxons with different polarities will attract each other, hence the possibility of forming a bound state which oscillates around the common center of mass. Breather type solutions are unstable with respect to damping, therefore the amplitude of oscillations will soon decay and their energy would be dissipated through resistive channels.

\subsection{PERTURBATIONAL ANALYSIS}

In the presence of a transport current, a Josephson fluxon will move under the action of Lorentz force. Due to Faraday law this motion generates an electric field which couples to the normal current and causes dissipation of energy. In equilibrium state where the amount of energy injection due to transport current is equal to dissipation caused by quasiparticle resistance, the velocity of a fluxon can be obtained using a perturbational analysis[62] in 
terms of transport current and viscous drag coefficient. A Hamiltonian can be written for the unperturbed SG in (45) as following[62]

$$
\mathcal{H}=\int_{-\infty}^{\infty}\left[\frac{\dot{\theta}^{2}}{2}+\frac{\theta^{\prime 2}}{2}+1-\cos \theta\right] d x
$$

from which the normalized energy of a single fluxon in (49) is given by

$$
E=\frac{8}{\sqrt{1-\left(v / c_{s}\right)^{2}}} .
$$

Treating the dissipative and transport terms in (44) as perturbations which act only upon the center of mass of fluxon, we obtain

$$
v= \pm \frac{c_{s}}{\sqrt{1+\left(\frac{4 \eta}{\pi \beta}\right)^{2}}}
$$

for the velocity of a fluxon in stationary state where the acting forces balance out[62].

\subsection{DYNAMICS OF SINGLE FINITE LENGTH LJJ}

Flux dynamics in LJJs is strongly affected by the junction geometry. The most extensively studied geometries are shown in Fig. 16. While the main governing equation is the same, there is a qualitative difference between linear junctions ( the first three ) with the annular junction. A major complication in the dynamics of linear (rectangular) finite length LJJs arises due to interaction of fluxons with the boundary conditions.

\subsubsection{ANNUALR JUNCTIONS}

For an annular junction in absence of magnetic field, assuming a uniform bias current is applied, the governing equation is (44) with the boundary condition which simply reflects the spatial periodicity of the junction:

$$
\theta(x, t)=\theta(x+L, t) \pm 2 n \pi
$$

where $n$ is an integer associated to the net number of fluxons present in the junction. In presence of magnetic field the right hand side of (44) is modified by the term $K \frac{\partial}{\partial x}(\mathbf{B} \cdot \mathbf{r})$ where $\mathbf{B}$ is the normalized magnetic field, $\mathbf{r}$ is a unit radial vector and $K$ is a coupling constant[63]. Although more complicated in terms of fabrication and control, the annular geometry facilitates the possibility of studying flux dynamics in absence of boundary reflection effects. 


\subsubsection{LINEAR JUNCTIONS}

The main difference in the electrodynamics of linear junctions shown in Fig. 16 comes from the manner that a bias current is applied. In the overlap geometry a uniform bias current is entered as the $\beta$ term in (44) while the application of bias current in the inline junction is non-uniform and happens through the boundary conditions. General boundary conditions in presence of magnetic field for linear junctions can be written as

$$
\theta^{\prime}(0, t)=\theta^{\prime}(L, t)=h
$$

where $h=H / \lambda_{J} J_{c}$ is the normalized magnetic field applied in the $y$ direction and can be also time-dependent. The normalization is such that in absence of bias current, $h=2$ is the static threshold for magnetic flux to penetrate into junction as a Josephson vortex.

In absence of magnetic field and presence of a bias transport current $0<\beta<1$, the solution for (44) with open-circuit boundary conditions at both ends of junction, $\theta^{\prime}(0, t)=$ $\theta^{\prime}(L, t)=0$, and small damping factor $\eta \ll 1$, is a spatially uniform constant phase $\theta=$ $\sin ^{-1}(\beta)$. If bias current exceeds the critical value, the solution becomes a time-varying still spatially uniform phase represented by the non-zero voltage curve in I-V characteristic of a typical LJJ. Reducing the bias current progressively back will slow down the phase evolution until at some point before a transition happens to zero-voltage state, the solution becomes dynamically unstable that it is possible for one or more fluxons to appear spontaneously inside the junction. Under the action of transport current the fluxon will move toward one of the edges and if energetic enough will get reflected back as anti-fluxon, changing the phase at the edges by $4 \pi$ each time a reflection happens. The resonating motion of a fluxon between the edges would result in a voltage step in I-V curve known as zero field steps [64]. Depending on the number of fluxons that resonate inside the junction the asymptotic voltage appear at regular intervals of $V_{\max }=n\left(\Phi_{0} c_{s} / L\right)$ in which the step index $n$ indicates the number of fluxons involved.

In presence of small magnetic field $H \ll H_{1}$, applied at the two edges of the junction, the current would enhance in one edge and reduce at the other. At right combination of parameters, the asymmetry would cause the traveling fluxons to get reflected with enhanced energy at the energy-adding edge and die at the energy-subtracting edge which gives rise to some of the energy reflecting back into junction in the form of small amplitude radiation which in turn travels toward the other edge and if the energy is sufficient enough will give birth to another fluxon. This is a new dynamic steady state which causes a $2 \pi$ phase change and results in voltage steps in I-V curve known as Fiske steps[65] which occur at one-half 
that of the first zero field steps, $V_{\max }=n\left(\Phi_{0} c_{s} / 2 L\right)$. Higher order Fiske steps also occur with more complicated dynamical structure.

When applied magnetic field exceeds the threshold of penetration at one of the edges, fluxons can penetrate and accelerate due to bias current toward the other edge where they might reflect back or exit the junction. Right combination of parameters would result in a dynamic steady state in which fluxons get injected continuously at one edge and exit through the other in a unidirectional motion causing another set of voltage steps in I-V curve known as flux-flow steps. The average voltage depend on the number of fluxons as well as their average speed of flow as can be approximated by $V=\mu_{0}\left(2 \lambda+d_{j}\right) H v$ in case of bulk electrodes where $H$ is the applied magnetic field and $v$ is the average velocity of the chain of flowing fluxons. This steady state is the basic operation mechanism of flux flow oscillators used in superconducting electronics[66, 67].

\subsection{SUMMARY}

The complex nonlinear dynamics of Josephson vortices in LJJs as well as the similarity of the behavior of GBs in SRF materials as both weak-link and strong-link Josephson junctions motivated us to pursue this topic further in specific geometries related to SRF cavities. As will be stated later in detail, the conventional theory of LJJs is not sufficient to describe the strong-link nature of GBs in materials like $\mathrm{Nb}$, therefore further investigations are required to tackle these problems. In the following chapters, we will present the original result of our simulation of the dynamics of vortices in weak-link and strong-link GBs by solving relevant governing equations. 


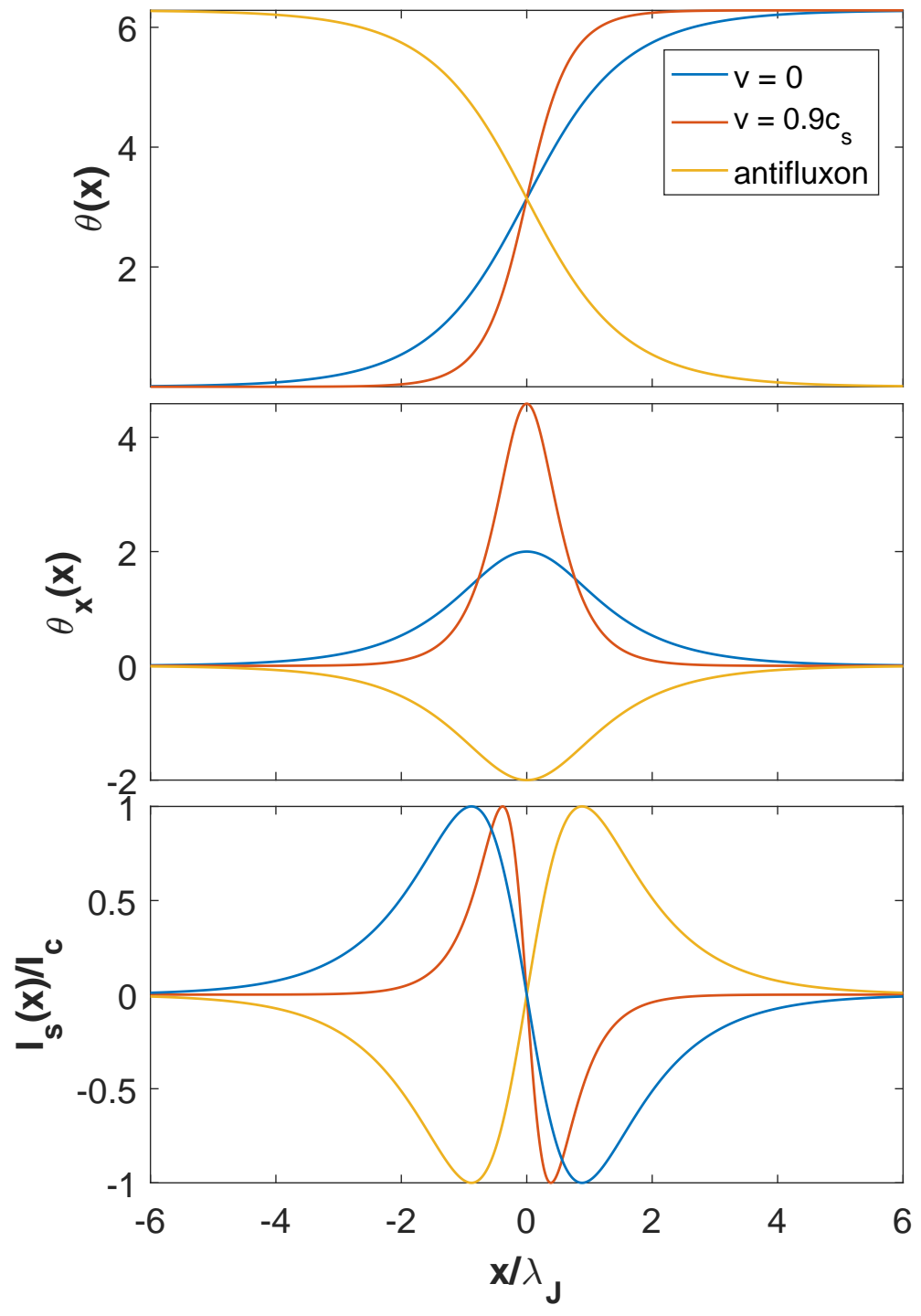

FIG. 18: Josephson vortex represented in terms of phase difference distribution $\theta(x)$, magnetic field distribution $\theta_{x}(x)$ and current distribution $I_{s}(x)=I_{c} \sin \theta(x)$, moving with different velocities. 


\section{CHAPTER 3}

\section{SIMULATION OF NONLINEAR DYNAMICS OF JOSEPHSON VORTICES IN WEAK-LINK GRAIN BOUNDARIES}

In this chapter we address a nonlinear electromagnetic response of a single LJJ across a film screen in a parallel field. We solved the sine-Gordon equation numerically to calculate the dynamics of penetration, annihilation and exit of Josephson vortices and antivortices oscillating under the dc and ac field.

Dynamics of Josephson vortices in long Josephson junctions (LJJs) under dc and ac magnetic fields has been the subject of much interest [5, 42, 43, 62, 64, 67, 68]. For instance, the barrier and overlap LJJs have been studied extensively for applications in superconducting electronics [42, 43], particularly flux flow oscillators [69, 70, 71, 72]. The electrodynamics of LJJ has attracted a renewed attention after the discovery of high- $T_{c}$ superconducting cuprates and iron based superconductors in which the grain boundaries between misoriented crystallites behave as long Josephson junctions which subdivide the materials into weakly coupled superconducting regions $[2,11]$. The latter gives rise to the electromagnetic granularity [73] which is one of the serious obstacles for applications of the cuprate and the iron-based superconductors [74].

Another situation in which the weak-linked grain boundaries becomes essential occurs in superconducting resonator cavities [6] in which the amplitudes of the radio-frequency $(\simeq 0.1-5 \mathrm{GHz})$ screening currents flowing at the inner surface of the cavity can approach the depairing current density $J_{d}$. In this case the grain boundaries even in such conventional materials as $\mathrm{Nb}$ can behave as LJJs [15, 16, 17, 25, 35], even though they do not manifest themselves as weak links in dc magnetization or transport properties at much lower dc currents $J \ll J_{d}$. It has been suggested that the Josephson vortices penetrating through grain boundaries can account for the linear decrease of the quality factors $Q(H)$ in $\mathrm{Nb}$ resonator cavities $[17,25,26]$. Understanding the electrodynamics of Josephson vortices in weak-linked grain boundaries requires addressing the following issues: 1 . The minimum amplitude of the ac field $H_{p}(\omega)$ the Josephson vortices start penetrating the LJJ and the relation between $H_{p}$ and the dc critical field. 2. The field dependence of the power $P(H)$ dissipated in the LJJ at $H>H_{p}$ and its contribution to the nonlinear surface resistance 
$R_{s}(H)$. 3. The effect of a finite length of the LJJ on $H_{p}$ and $P(H)$ which would account for a finite grain size in polycrystalline materials of a finite film thickness in a screen.

\subsection{ELECTRODYNAMICS}

We consider a LJJ perpendicular to a flat screen of width $L$ as shown in Fig. 19. The uniform magnetic field $H(t)$ is applied along the $y$-axis parallel to one side of the screen at $x=0$. At the other side of the screen we assume the boundary condition $H(L, t)=0$. The LJJ is described by the partial differential equation in (43) (except that here $\beta=0$ ) and the local field distribution along the LJJ $B(x, t)=\left(\Phi_{0} / 4 \pi \lambda\right) \theta^{\prime}(x, t)$ defines the boundary conditions at $x=0$ and $x=L$ :

$$
\theta^{\prime}(0, t)=\frac{2 \pi \mu_{0} d}{\Phi_{0}} H(t), \quad \theta^{\prime}(L, t)=0 .
$$

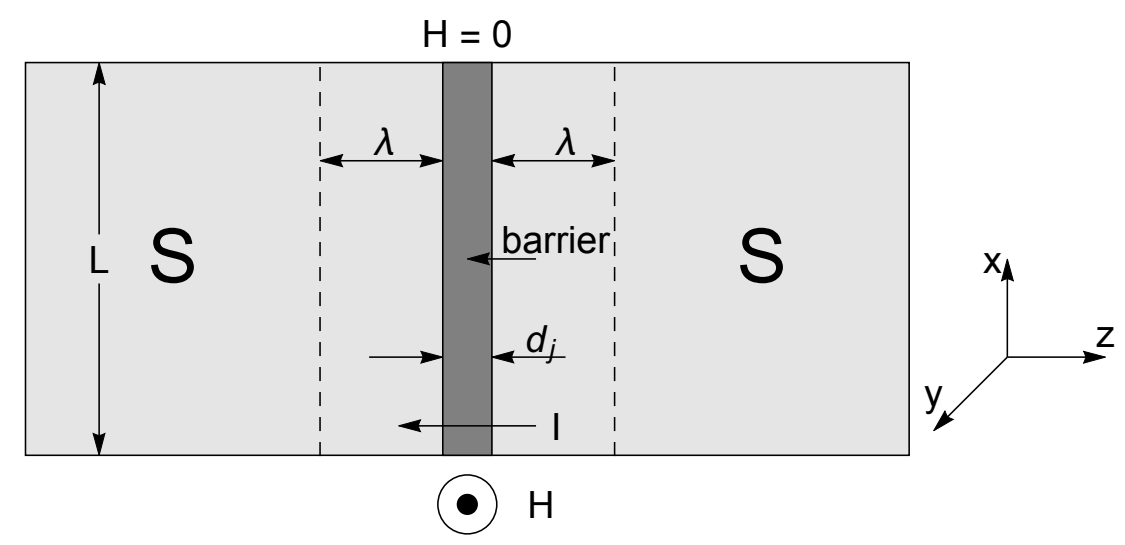

FIG. 19: Geometry of a long Josephson junction in a film which screens the uniform magnetic field $H$ applied in the region $x<0$.

As an illustration, we estimate $\omega_{J}$ and $\omega_{c}$ for $\mathrm{Nb}$ at different ratios of $J_{c} / J_{d}$ where $J_{d}=\Phi_{0} / 3 \sqrt{3} \pi \mu_{0} \lambda^{2} \xi$ is the bulk depairing current density, and $\xi$ is the coherence length. Taking $\lambda \approx \xi \approx 40 \mathrm{~nm}$, the typical excess grain boundary resistance $R_{j}=2 \times 10^{-13} \Omega \mathrm{m}^{2}$ for $\mathrm{Nb}$ [75], and $C=\epsilon_{0} \epsilon_{r} / d_{j}$ where $\epsilon_{r} \simeq 3$ is the static dielectric constant of filled electron bands and $d_{j} \simeq 1 \mathrm{~nm}$ is the atomic width of the grain boundary, we obtain $J_{d} \simeq 150$ $\mathrm{MA} / \mathrm{cm}^{2}$, and $R_{j} J_{d} \simeq 0.3 \mathrm{~V}$. Then $\omega_{c} \simeq 10^{15}\left(J_{c} / J_{d}\right) \mathrm{Hz}$, and $\omega_{J} \simeq 4 \cdot 10^{14}\left(J_{c} / J_{d}\right)^{1 / 2} \mathrm{~Hz}$. The McCumber parameter $\beta_{c}=\left(\omega_{c} / \omega_{J}\right)^{2} \simeq 6 J_{c} / J_{d}$ defines the effect of dissipation for a steadystate propagation of Josephson vortices; the case of $\beta_{c} \ll 1$ corresponds to the overdamped limit in which dissipative ohmic currents dominate over the displacement currents described by the inertial term $\propto \partial^{2} \theta / \partial t^{2}$ in (43). The Josephson weak link is by definition an interface 
with $J_{c} \ll J_{d}$ so, for the above numbers, the grain boundaries would be in the overdamped limit. However, $J_{c}$ across grain boundaries in $\mathrm{Nb}$ can be very high and close to $J_{d} \xi / \lambda$ in which case 43 is no longer valid and the equation for $\theta(x, t)$ becomes nonlocal particularly in materials with large G-L parameter $\kappa[76,77,78]$. In this section we only consider the local Josephson limit described by 43 both for $\beta_{c}>1$ and $\beta_{c}<1$.

As will be shown below, penetration and annihilation of Josephson vortices can result in significant instant power dissipation $P(t)=\int_{0}^{L} V(x, t) J(x, t) d x$ per unit height of the junction along the $y$-axis, where $V=\left(\Phi_{0} / 2 \pi\right) \partial \theta / \partial t$ is the voltage, and $J(x, t)$ is a sum of the Josephson, ohmic and displacement current densities:

$$
P(t)=\frac{\Phi_{0} J_{c}}{2 \pi} \int_{0}^{L} d x\left[\sin \theta+\frac{1}{\omega_{c}} \frac{\partial \theta}{\partial t}+\frac{1}{\omega_{J}^{2}} \frac{\partial^{2} \theta}{\partial t^{2}}\right] \frac{\partial \theta}{\partial t} .
$$

In a periodic ac field with frequency $\omega$ the contributions of Josephson and displacement currents vanish after averaging over the ac period $T=2 \pi / \omega$. As a result, the average power is caused only by the ohmic currents:

$$
\bar{P}=\frac{\Phi_{0} J_{c}}{2 \pi \omega_{c} T} \int_{t_{0}}^{t_{0}+T} d t \int_{0}^{L}\left(\frac{\partial \theta}{\partial t}\right)^{2} d x .
$$

In dimensionless form the governing equation becomes

$$
\ddot{\theta}+\eta \dot{\theta}=\theta^{\prime \prime}-\sin \theta
$$

where the boundary conditions and instant power takes the form

$$
\begin{array}{r}
\theta^{\prime}(0, t)=h, \quad \theta^{\prime}(l, 0)=0 \\
P / P_{0}=\int_{0}^{l} d x[\sin \theta+\eta \dot{\theta}+\ddot{\theta}] \dot{\theta}
\end{array}
$$

where $l=L / \lambda_{J}, h=\left(2 \pi \mu_{0} d \lambda_{J} / \Phi_{0}\right) H(t)$ and $P_{0}=c_{s} \Phi_{0} J_{c} / 2 \pi$.

\subsection{FIELD}

We first consider the overdamped limit in which the term $\propto \ddot{\theta}$ can be neglected and (58) turns into a nonlinear diffusion equation,

$$
\dot{\theta}=\theta^{\prime \prime}-\sin \theta
$$

where the overdot is renormalized in units of $\omega_{c}$. Solutions of (61) are shown in Fig. 20 for $L=20 \lambda_{J}$ and different magnetic fields $H_{d c}$. Figure 20a shows the metastable Meissner state 
at $H_{c 1 J}<H_{d c}<H_{1}$, in which the magnetic flux is screened at the edge over the length $\sim \lambda_{J}$. Figure 20b shows the case of $H_{d c}=H_{1}$ at which the first vortex nucleates at the edge and accelerates until the velocity becomes limited by the friction force of ohmic currents. Figure 20c shows the flux flow state at higher field $H_{d c}=5 H_{1}$ at which vortices periodically enter, travel all the way to the other end at $x=L$ where they disappear. This dynamic state is characteristic of a thin film screen [13] in which the LJJ provides a path for constant flux pumping from the region of applied field to the inner region of $H=0$, unlike a stationary chain of Josephson vortices in a uniform field which is the same at both edges of the junction $[42,43]$.

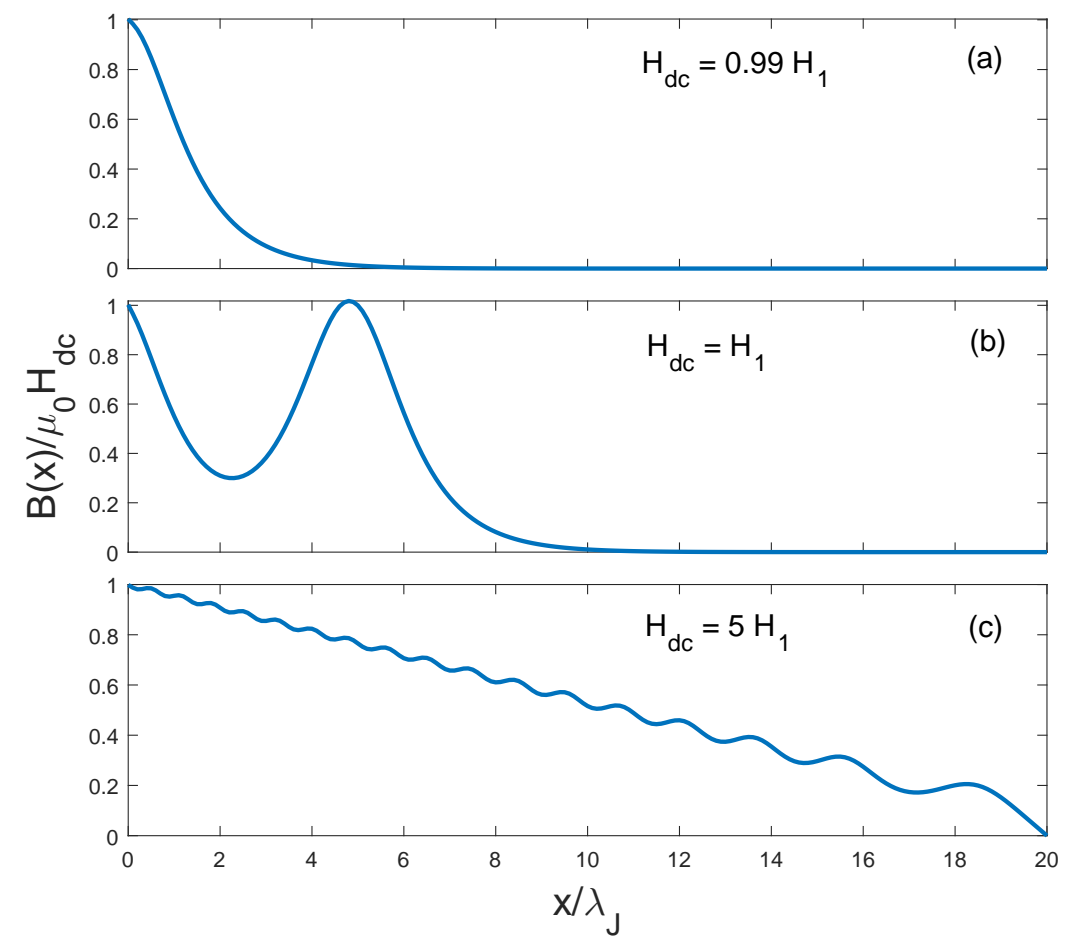

FIG. 20: Profiles of the local magnetic field $B(x)$ in a LJJ at $L=20 \lambda_{J}$ solution of (61) and different values of $H_{d c}$.

If we solve (58) in full without neglecting the inertial term, as solutions shown in Fig. 21 indicate, the junction will be in overdamped regime and the assumption of (61) is justified even for values of $\eta \sim 1$.

Figure 22 shows the evolution of the local field $B(x, t)$ along the LJJ and the instant power $P(t)$ at $H_{d c}=2 H_{1}$ and $\eta=1$. One can see that each penetration and annihilation of vortices at the edges produces peaks in $P(t)$. The highest peak in $P(t)$ occurs during penetration of the first vortex at $x=0$ after the field was turned on and the vortex is 


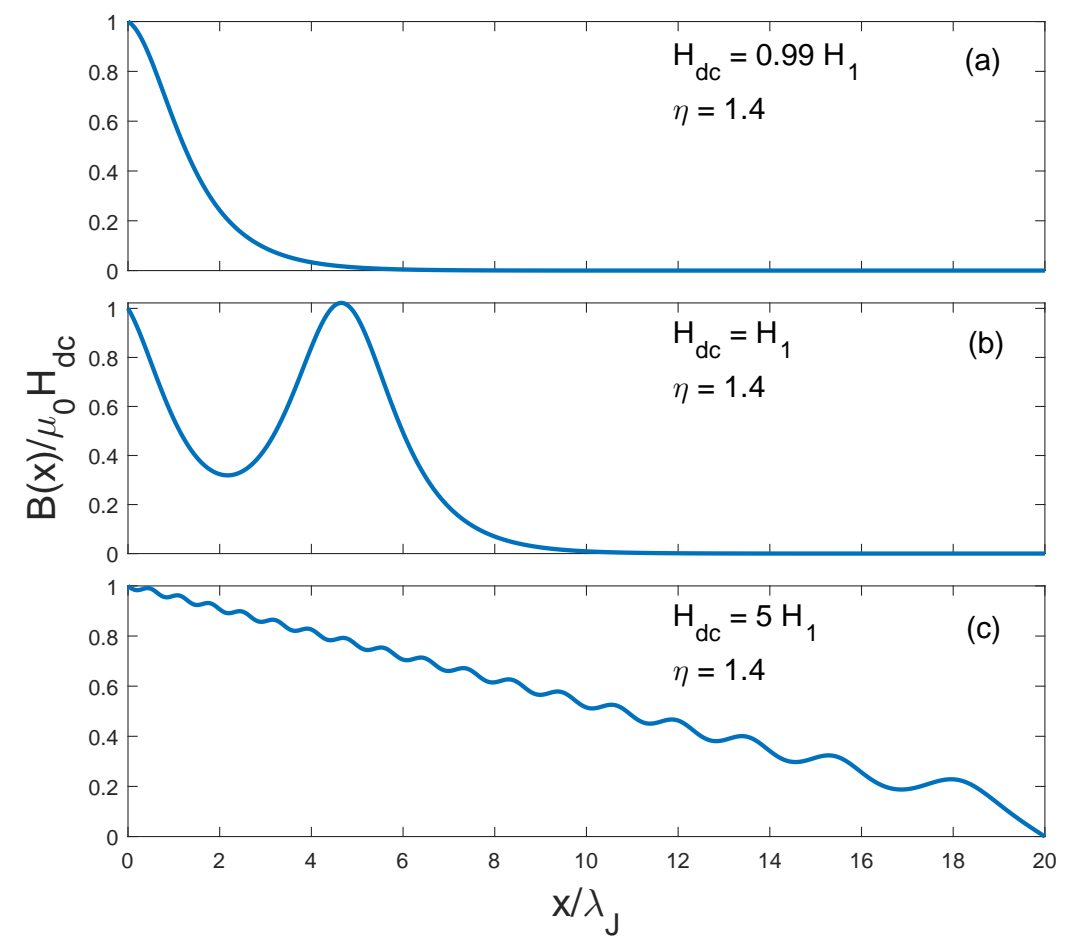

FIG. 21: Profiles of the local magnetic field $B(x)$ in a LJJ at $L=20 \lambda_{J}, \eta=1.4$ and different values of $H_{d c}$.

accelerated strongly by the Lorentz force of screening current. After penetration of several vortices, the Lorentz force which pushes the next vortex in the junction is reduced by the counterflow of vortices already in the LJJ, so the peaks in $P(t)$ caused by penetrating vortices are reduced. As the vortex exits the junction at $x=L$, it is accelerated again due to attraction to its antivortex image [62], producing peaks in $P(t)$.

The effects of displacement currents on dynamics of Josephson vortices will show up first by solving (58) for moderate damping at $\eta=0.3$. The results shown in Fig. 23 are calculated at $H_{d c}=2 H_{1}$ and indicate that in this case vortices gain some inertia and upon reaching the edges dissipate most of their energy, but a small part of it would get reflected in the form of decaying electromagnetic waves back to the junction.

For weaker damping $(\eta<0.1)$, vortices move with a nearly uniform velocity until they get reflected from the edge of the junction without losing much of their energy but reversing their polarity and velocity[64]. As shown in Fig. 24, for $\eta=0.01$ and $H_{d c}=1.2 H_{1}$, vortices move almost with their initial velocity but upon reaching the edge of the junction at $x=L$, they get reflected as anti-vortices. The reflected anti-vortices pass through incoming vortices[68] causing only small amount of dissipation. The multiple reflections of vortices from the edges 


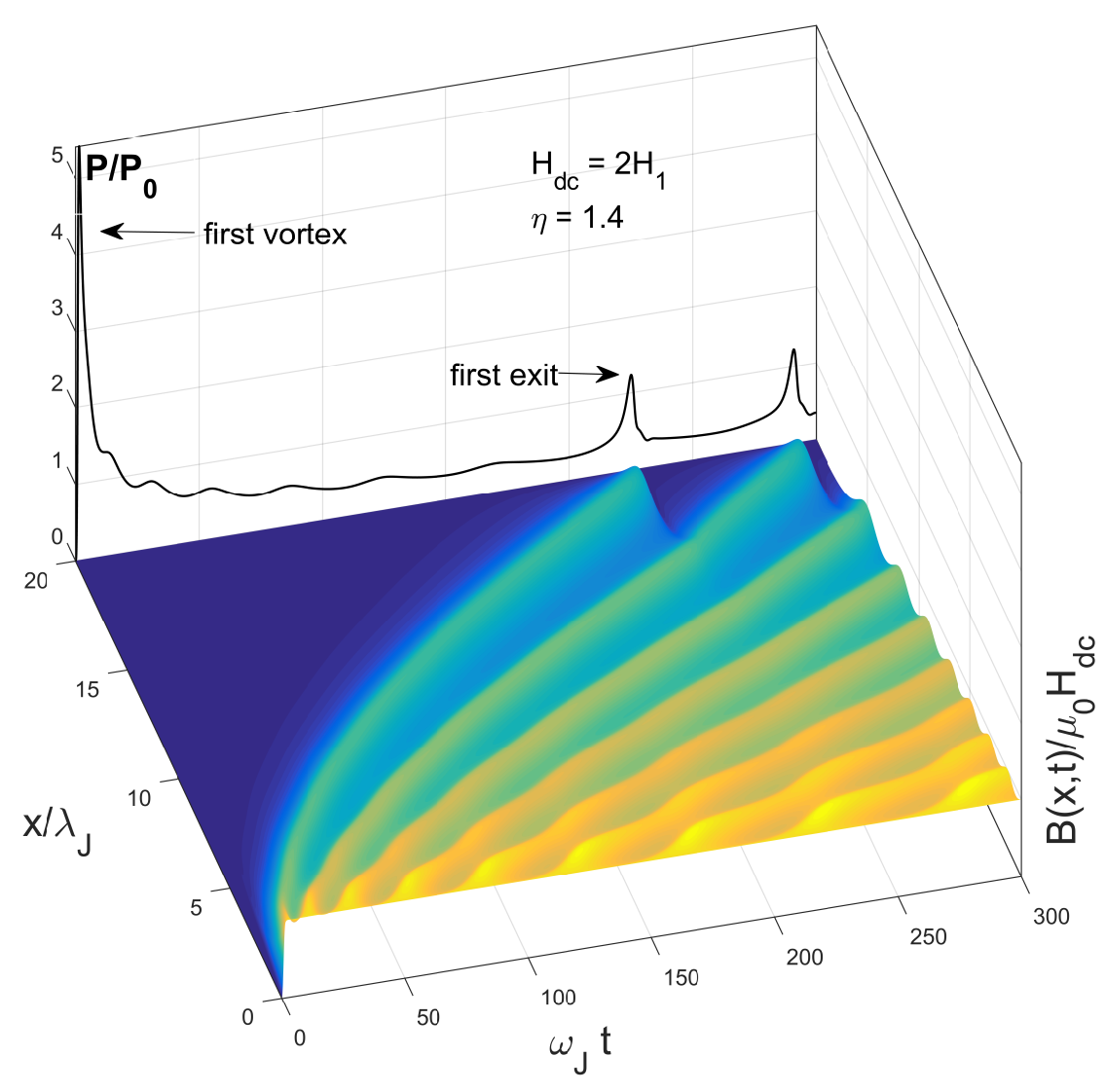

FIG. 22: Evolution of the local magnetic field $B(x, t)$ along the LJJ, and the instant power $P(t)$ calculated for $H_{d c}=2 H_{1}$. Results of solution of $(58)$ in the overdamped limit at $\eta=1.4$

along with continuous pumping of the electromagnetic waves can result in a chaotic behavior of $\theta(x, t)$ which we do not address in this work.

Shown in Fig. 25 is the averaged power $\bar{P}$ generated by moving Josephson vortices calculated from $(58)$ at $\eta=0.2$. At high fields $\left[H_{d c}>(3-4) H_{1}\right]$, the dependence $\bar{P}\left(H_{d c}\right)$ becomes nearly quadratic in $H_{d c}$ but at lower fields, there are step-like features in $\bar{P}\left(H_{d c}\right)$ associated with penetration of Josephson vortices. From the power dissipation relation $\bar{P}=$ $I^{2} R_{f}$ where $I=H_{d c}$ is the total current flowing along the screen, we calculate the field dependence of the flux flow resistance $R_{f}\left(H_{d c}\right)$ plotted in inset of Fig. 25. Here $R_{f}\left(H_{d c}\right)$ vanishes at $H_{d c}=H_{1}$ and increases with $H_{d c}$, approaching the total LJJ resistance $R_{0}=$ $R_{j} / L$ at $H_{d c}>4 H_{1}$.

\subsection{SINGLE-MODE AC FIELD}

In this section we consider a LJJ under a single-mode ac magnetic field, $H=H_{a c} \sin \omega t$. 


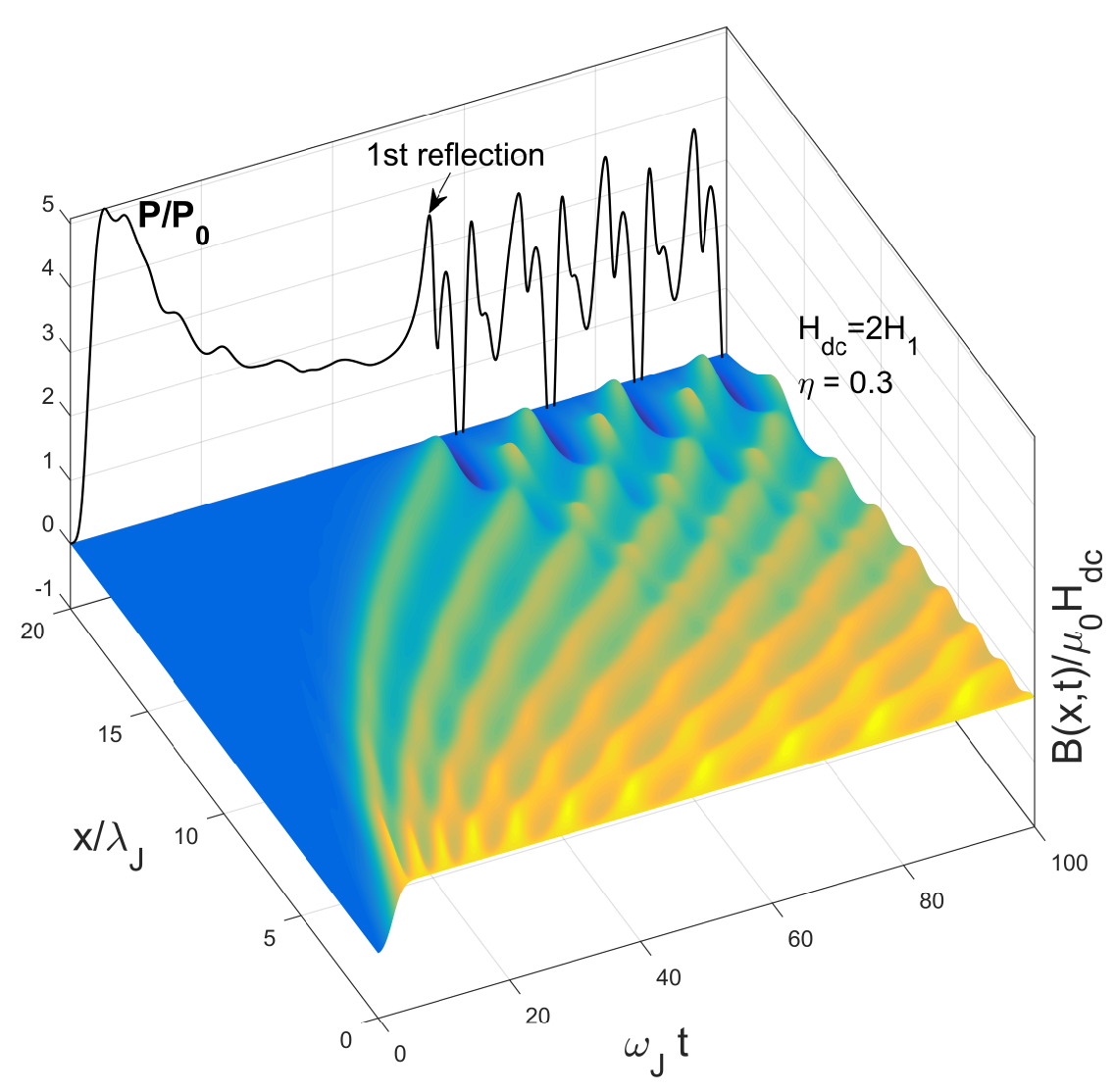

FIG. 23: Evolution of the local magnetic field $B(x, t)$ along the LJJ, and the instant power $P(t)$ calculated for $H_{d c}=2 H_{1}$. Results of solution of 58 for a moderately dissipative case of $\eta=0.3$. Standing electromagnetic waves generated by moving vortices in the LJJ manifest themselves in "ripple" on $B(x, t)$ and in a more complex behavior of $P(t)$ than for the overdamped limit.

In this case it is more convenient to rescale the time in the units of the characteristic frequency $t \rightarrow \omega_{c} t$, so that the dimensionless sine-Gordon equation takes the form

$$
\beta \ddot{\theta}+\dot{\theta}=\theta^{\prime \prime}-\sin \theta
$$

where $\beta=\left(\omega_{c} / \omega_{J}\right)^{2}$. The boundary conditions become

$$
\theta^{\prime}(0, t)=h_{a c} \sin \alpha t, \quad \theta^{\prime}(l, t)=0
$$

where $\alpha=\omega / \omega_{c}$ and $h_{a c}=\left(2 \pi \mu_{0} d \lambda_{J} / \Phi_{0}\right) H_{a c}$. The instant power is then

$$
P / P_{0}=\int_{0}^{l} d x[\sin \theta+\dot{\theta}+\beta \ddot{\theta}] \dot{\theta}
$$

where $P_{0}=\Phi_{0} J_{c} \lambda_{J} \omega_{c} / 2 \pi$. 


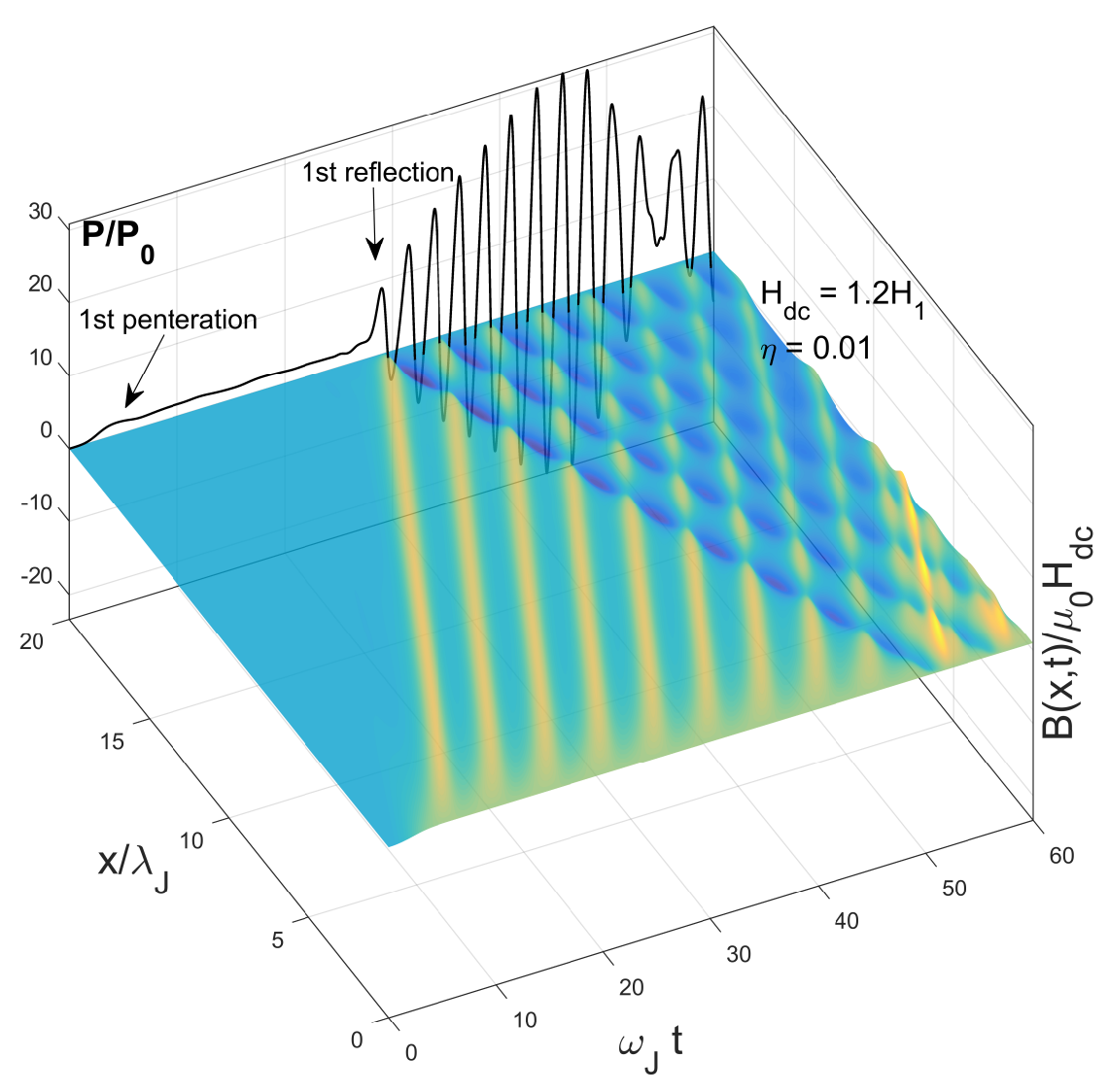

FIG. 24: Evolution of the local magnetic field $B(x, t)$ calculated for a weakly dissipative case of $\eta=0.01$ at $H_{d c}=1.2 H_{1}$. Vortices undergo multiple reflections from the edges with the reversal of their polarity and velocity.

As was shown above, the plasma frequency for the grain boundaries in $\mathrm{Nb}$ is typically in the infrared region $\left(\omega_{J} \sim 10^{12}-10^{14} \mathrm{~Hz}\right)$ so for many microwave and rf applications $(\omega \sim 0.1-10 \mathrm{GHz})$, the parameter $\beta \ll 1 / \alpha\left(\right.$ i.e. $\left.\omega \omega_{c} \ll \omega_{J}^{2}\right)$ is negligible and (62) reduces to (61). However, to have a rough estimation of how much each effect would contribute to power dissipation we can represent the unit of dissipated power $P_{0}$ as:

$$
P_{0}=\lambda R_{j} J_{c}^{2} \sqrt{J_{d} / \kappa J_{c}}
$$

which depends on characteristic properties of grain boundaries such as specific resistance $R_{j}$ and critical current density $J_{c}$. For Nb with $\lambda=40 \mathrm{~nm}$ and $\kappa=1$ using $R_{j}=2 \cdot 10^{-13} \Omega \mathrm{m}^{2}$ from previous calculation, we obtain $P_{0}(N b)=0.018\left(J_{c} / J_{d}\right)^{3 / 2}($ Watt $/ \mu \mathrm{m})$. Finally, for a grain boundary of $J_{c}=0.1 J_{d}$ and height $\sim 0.1 \mu \mathrm{m}$, the amount of unit power dissipated is $\simeq 57 \mu$ Watt. The size of the GB considered in this simulation is $20 \lambda_{J}$ which using the above parameters for a $\mathrm{GB}$ in $\mathrm{Nb}$ translates to a size about $2.5 \mu \mathrm{m}$ which is reasonable. Same type 


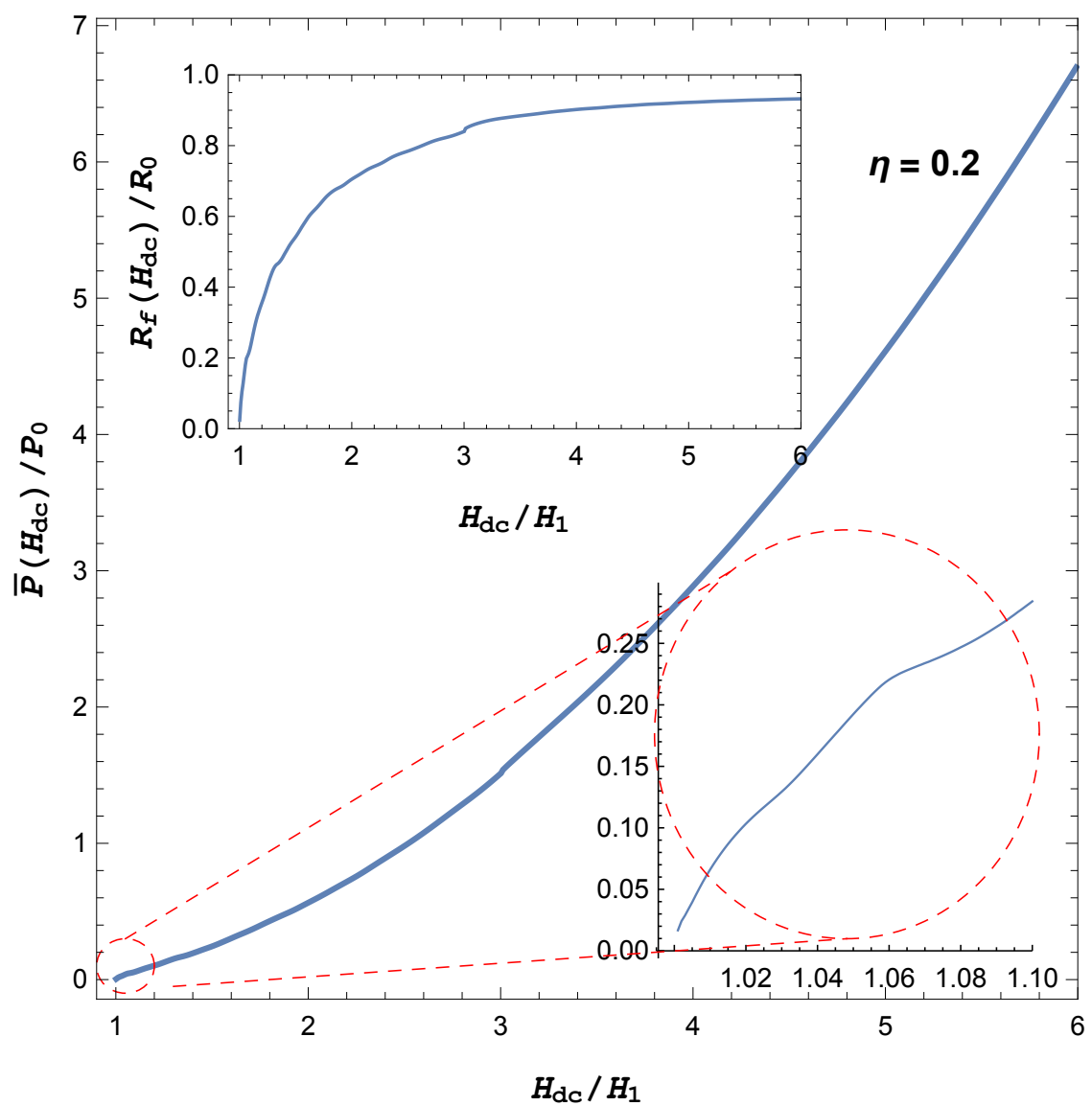

FIG. 25: Averaged power $\bar{P} / P_{0}$ as a function of reduced dc magnetic field $H_{d c} / H_{1}$ calculated for $\eta=0.2$. Inset shows the flux flow resistance as a function of $H_{d c} / H_{1}$ where $R_{0}=R_{j} / L$ is the total quasiparticle resistance of the junction.

of calculations for $\mathrm{Nb}_{3} \mathrm{Sn}$ with $\lambda=100 \mathrm{~nm}$ and $\kappa=20$ leads to even larger dissipation as $R_{j}$ in $\mathrm{Nb}_{3} \mathrm{Sn}$ is several orders of magnitude larger than that of a $\mathrm{Nb}$ grain boundary.

Our numerical simulations of (61) associated with boundary conditions in (63) have shown that it has a solution $\theta(x, t)$ with the periodicity of the applied ac field. Shown in Fig. 26 are the profiles of magnetic field just before and after penetration of a vortex calculated for $\alpha=0.01$. These snapshots of $B(x, t)$ at different times and $H_{a c} \approx H_{1}$ suggest that a vortex (or antivortex during the negative field cycle) get trapped at the edge of the junction, just because vortices under oscillating ac field have limited time to enter the junction. As a result, the threshold field $H_{p}(\omega)$ of vortex penetration becomes larger than $H_{1}$ and increases with the frequency, so that there is enough time during the part of the period when $H_{a c}|\sin \omega t|>H_{1}$ for the vortex to penetrate by the distance $\sim \lambda_{J}$. Calculations of $\bar{P}$ given below show that 
the rf power dissipated in the LJJ increases sharply at $H_{a c}>H_{p}$.

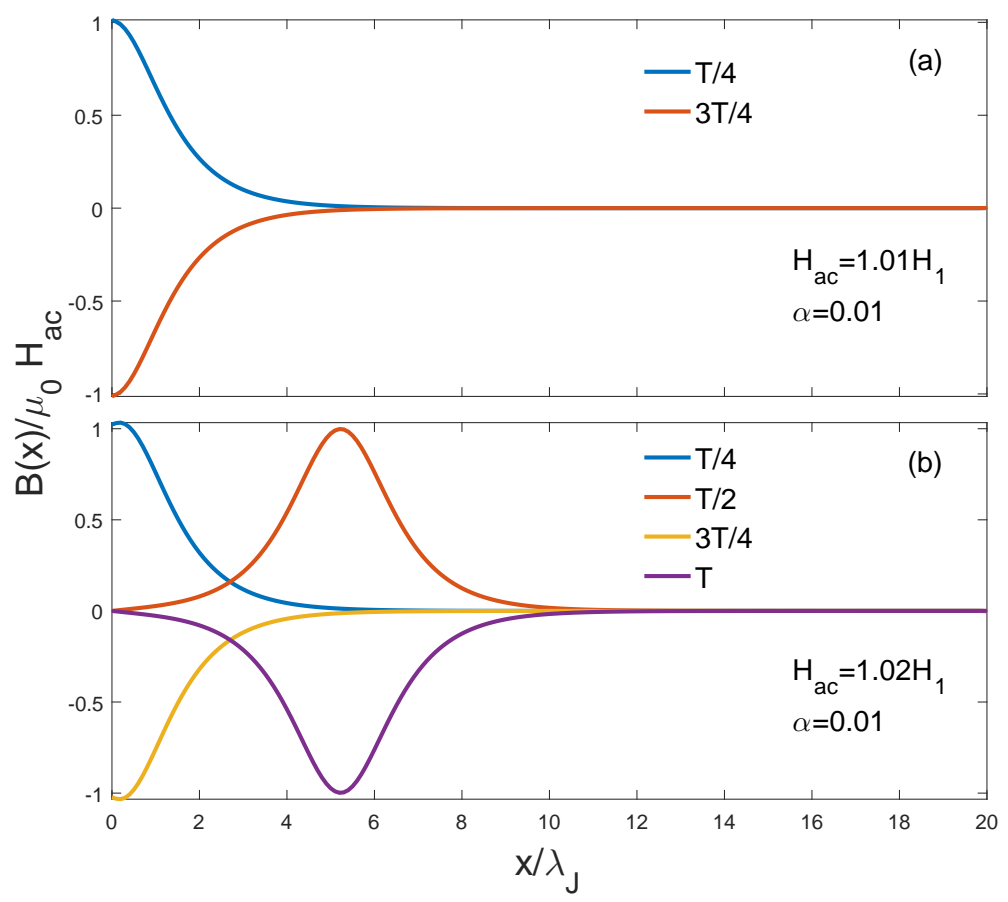

FIG. 26: Snapshots of magnetic field profiles $B(x)$ calculated for $\alpha=0.01$ at different times: (a) just before the first vortex penetrates; (b) just after the penetration of the first vortex/anti-vortex occurred. Arrows show whether the applied field $H(t)$ is increasing or decreasing.

Unlike the unidirectional flow of vortices under de field considered in section 3.2, the flux dynamics under ac field includes penetration of Josephson vortices during the positive ac cycle followed by penetration of antivortices during the negative cycle and their subsequent annihilation. Shown in Figs. 27-28 are representative examples of the evolution of the local magnetic field $B(x, t)$, and the corresponding instant power $P(t)$ plotted for a full ac cycle calculated from (62) at $H_{a c}=2 H_{1}$. In the particular case of overdamped flux dynamics shown in Fig. 27, about eight vortices penetrate the LJJ during the positive ac cycle, giving rise to small peaks in $P(t)$. Of these vortices, the first three annihilate upon collisions with residual anti-vortices generated during the previous negative half cycle, while the fourth one goes all the way along the junction and exits at the other end. The last four vortices do not reach the end of the junction and turn around as $H(t)$ changes sign; the very last vortex exits before antivortices appear, but three other vortices annihilate on their way back with incoming anti-vortices generated during the negative ac cycle. The same process repeats for antivortices during the negative cycle. Notice that annihilation of vortices and antivortices inside the junction results in peaks in $P(t)$ that are significantly higher than the peaks in 
$P(t)$ during penetration or exit of vortices at the edges.

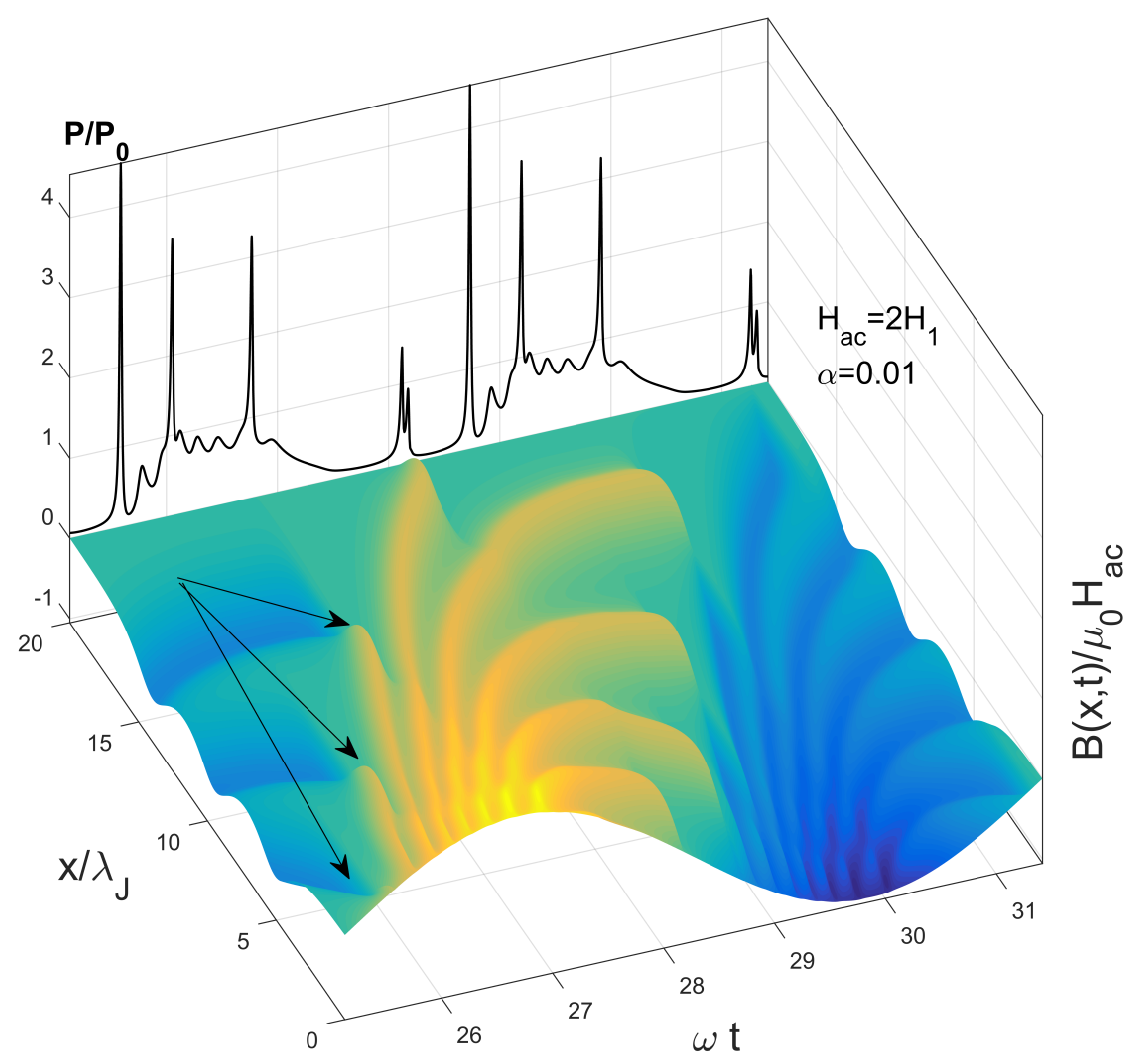

FIG. 27: Evolution of the local magnetic field $B(x, t)$ and the instant dissipated power calculated from (62) for $H_{a c}=2 H_{1}$. Overdamped limit at $\alpha=0.01$. Arrows show the points of annihilation of vortices and antivortices.

Fig. 28 shows the effect of displacement currents on the flux dynamics in a moderately overdamped limit at $\beta \ll 1 / \alpha$. We found that if $\alpha \beta \lesssim 0.1$, the response of the junction to the ac field remains periodic and similar to the solutions at $\beta \rightarrow 0$, except for generation of electromagnetic waves by accelerating/decelerating vortices upon interaction with boundaries and other vortices. As shown in Fig. 28, the number of vortices does not change as compared to Fig. 27, but because they now have some inertia, two vortices are able to reach the edge and leave behind weak electromagnetic radiation which manifests itself in "ripples" on $B(x, t)$ and a more irregular behavior of $P(t)$.

In the case of $\alpha \beta \sim 1$ shown in Fig. 29 vortices quickly enter the junction during the positive ac cycle and move with a nearly uniform velocity until they hit the other edge. There they get reflected from the edge as anti-vortices which then collide with newly entered anti-vortices, giving rise to local spikes of high magnetic field inside the junction before passing through each other and making their trip toward the other edge. In this regime, 


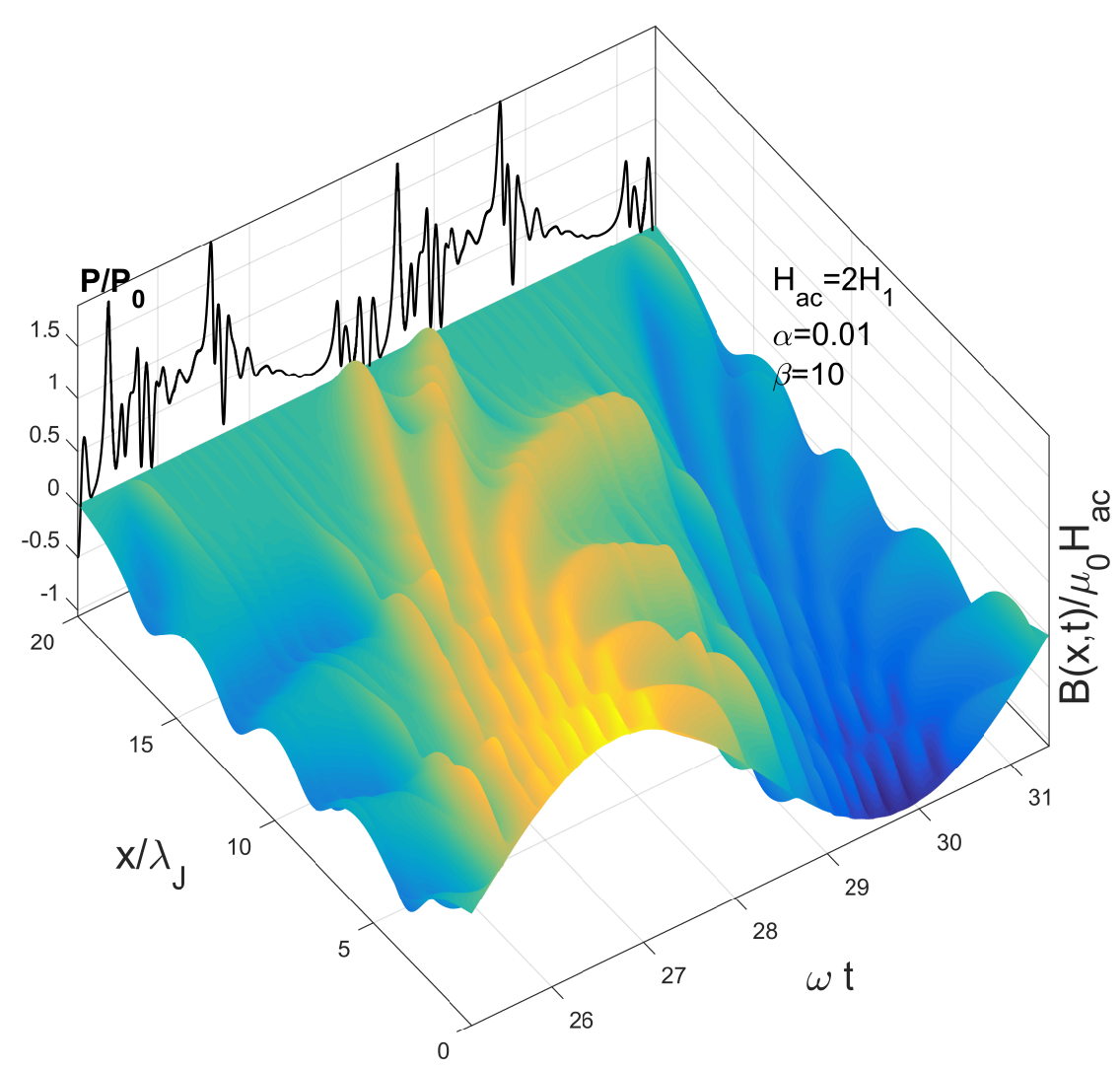

FIG. 28: Evolution of the local magnetic field $B(x, t)$ and the instant dissipated power calculated from (62) for $H_{a c}=2 H_{1}$. Moderately overdamped limit at $\alpha=0.01$ and $\beta=10$. The flux dynamics is similar to Fig. 27 except the additional "ripples" on $B(x, t)$ due to electromagnetic waves generated because of the effect of vortex inertia.

vortex dynamics is getting more chaotic as depicted in Fig. 29a; after several reflections, vortices eventually lose their energy due to ohmic losses and exit. Figure 29b illustrates a more chaotic behavior at $\alpha \beta>1$; here vortices undergo more reflections and less dissipation, forming a dynamic pattern in which twice in every ac period, half of the junction is filled with vortices and half with anti-vortices.

\subsubsection{DISSIPATED POWER}

We now calculate the mean dissipated power $\bar{P}$ in the overdamped limit $(\alpha \beta \ll 1)$, by averaging (57) over the ac period:

$$
\bar{P} / P_{0}=\frac{\alpha}{2 \pi} \int_{0}^{2 \pi / \alpha} d t \int_{0}^{l} \dot{\theta}^{2} d x,
$$

where $P_{0}=\Phi_{0} J_{c} \lambda_{J} \omega_{c} / 2 \pi$. Plotted in Fig. 30a is $\bar{P}$ as a function of ac field amplitude 

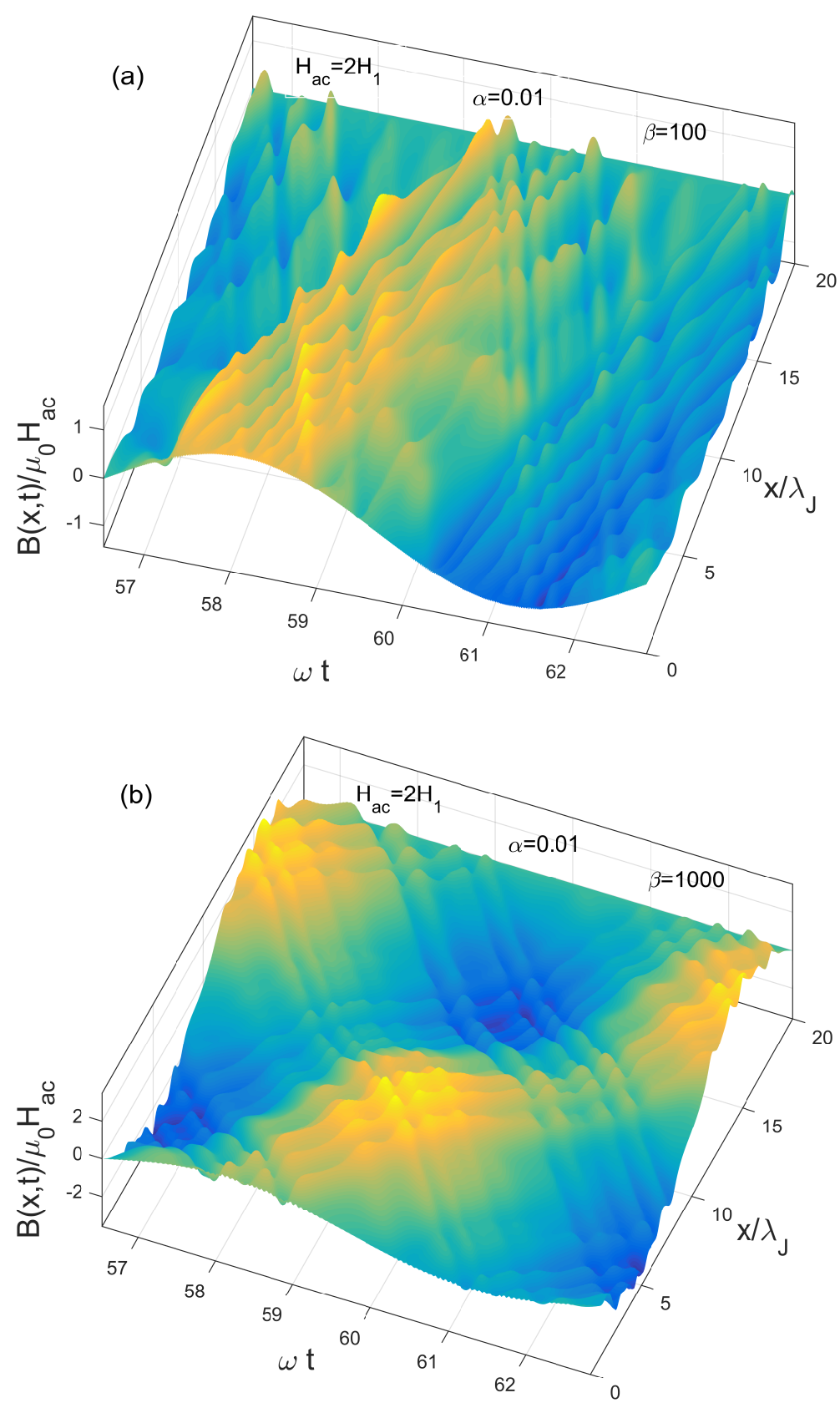

FIG. 29: Evolution of the local magnetic field $B(x, t)$ at $H_{a c}=2 H_{1}$, and $\alpha=0.01$ for different values of $\beta$ : (a) $\beta=100$; (b): $\beta=1000$. In both cases the ripples on $B(x, t)$ is due to standing electromagnetic waves generated by accelerating/decelerating vortices. In a weakly dissipative case shown in (b), vortex/anti-vortex bundles form during each half cycle. 
for different values of the dimensionless frequency $\alpha=\omega / \omega_{c}$ in the overdamped limit. One can clearly see steps in $\bar{P}\left(H_{a c}\right)$ due to the change of the mean number of vortices in the junction as $H_{a c}$ increases. For smaller frequencies $\alpha$, the steps are sharper and decrease in amplitude as $H_{a c}$ increases. As $\alpha$ increases, the sharp steps become broader until they disappear completely and $\bar{P}\left(H_{a c}\right)$ quickly becomes quadratic in $H_{a c}$.

It is instructive to express $\bar{P} / s=R_{s} I_{a}^{2} / 2$ in terms of the surface resistance $R_{s}$ for a stack of parallel LJJ spaced by $s$ along the $z$-axis, where $I_{a}=H_{a c}$ is the amplitude of the ac current flowing through the LJJ. The field dependence of $R_{s}\left(H_{a c}\right)=2 \bar{P}\left(H_{a c}\right) / s H_{a c}^{2}$ inferred from the above results for $\bar{P}\left(H_{a c}\right)$, is shown in Fig. 30(b). Several features of $R_{s}\left(H_{a c}\right)$ should be mentioned. First, $R_{s}\left(H_{a c}\right)$ increases sharply above a threshold field $H_{p}(\omega)$ which we associate with the field onset of penetration of Josephson vortices in the junction. At small frequencies, $\alpha=\omega / \omega_{c} \ll 1$, the dependence $R_{s}\left(H_{a c}\right)$ has a significant steplike feature component in which each step results from the change of the mean number of vortices in the LJJ by one as $H_{a c}$ increases. At higher frequencies, the steps $R_{s}\left(H_{a c}\right)$ become less pronounced and disappear at $\alpha>1$. For $H_{a c} \gg H_{1}$ the resistance approaches a constant value which, for an infinite LJJ, is just the surface resistance $R_{0}=\left(\mu_{0} R_{j} \omega d / 2\right)^{1 / 2} / s$ under the normal skin effect [38]. However, in our case of the LJJ of finite length $\left(L=20 \lambda_{J}\right)$, the asymptotic value of $R_{s}\left(H_{a c}\right)$ is smaller than $R_{0}$ obtained above. The value used in calculation and shown on Fig.30b corresponds to $R_{0}=2 R_{j} / \lambda_{J}$. Results similar to those shown in Fig. 30b were previously obtained by McDonald and Clem [38] and by Zhai et al. [39]. The frequency dependence of $\bar{P}\left(H_{a c}, \omega\right)$ at $H_{a c}=4 H_{1}$ shown in Fig. 31 , is close to the square root behavior. The latter is not surprising given that at $H_{a c}=4 H_{1}$, the Josephson vortices in the LJJ overlap and $R_{s}\left(H_{a c}\right)$ shown in Fig. 30b approaches the surface impedance of a normal conductor.

\subsubsection{FREQUENCY DEPENDENCE OF PENETRATION FIELD}

The field onset of sharp increase of the dissipated power $\bar{P}\left(H_{a c}\right)$ at which the first fluxon penetrates the junction increases with the frequency of the applied field, as it is evident from Fig. 30a. The calculated frequency dependence of $H_{p}(\omega)$ in the overdamped regime is shown in Fig. 32. Here the function $H_{p}(\omega)$ first increases linearly with $\omega$ at $\omega \ll \omega_{c}$ and then exhibits a faster increase with a downward curvature above $\alpha \sim 0.1$. At $\omega \ll \omega_{c}$, the penetration field can be approximated by

$$
H_{p}(\omega) \simeq H_{1}\left(1+1.4 \omega / \omega_{c}\right), \quad \omega \ll \omega_{c}
$$

These results show that $H_{p}$ is close to the dc superheating field of the junction if $\omega \ll \omega_{c}$. 

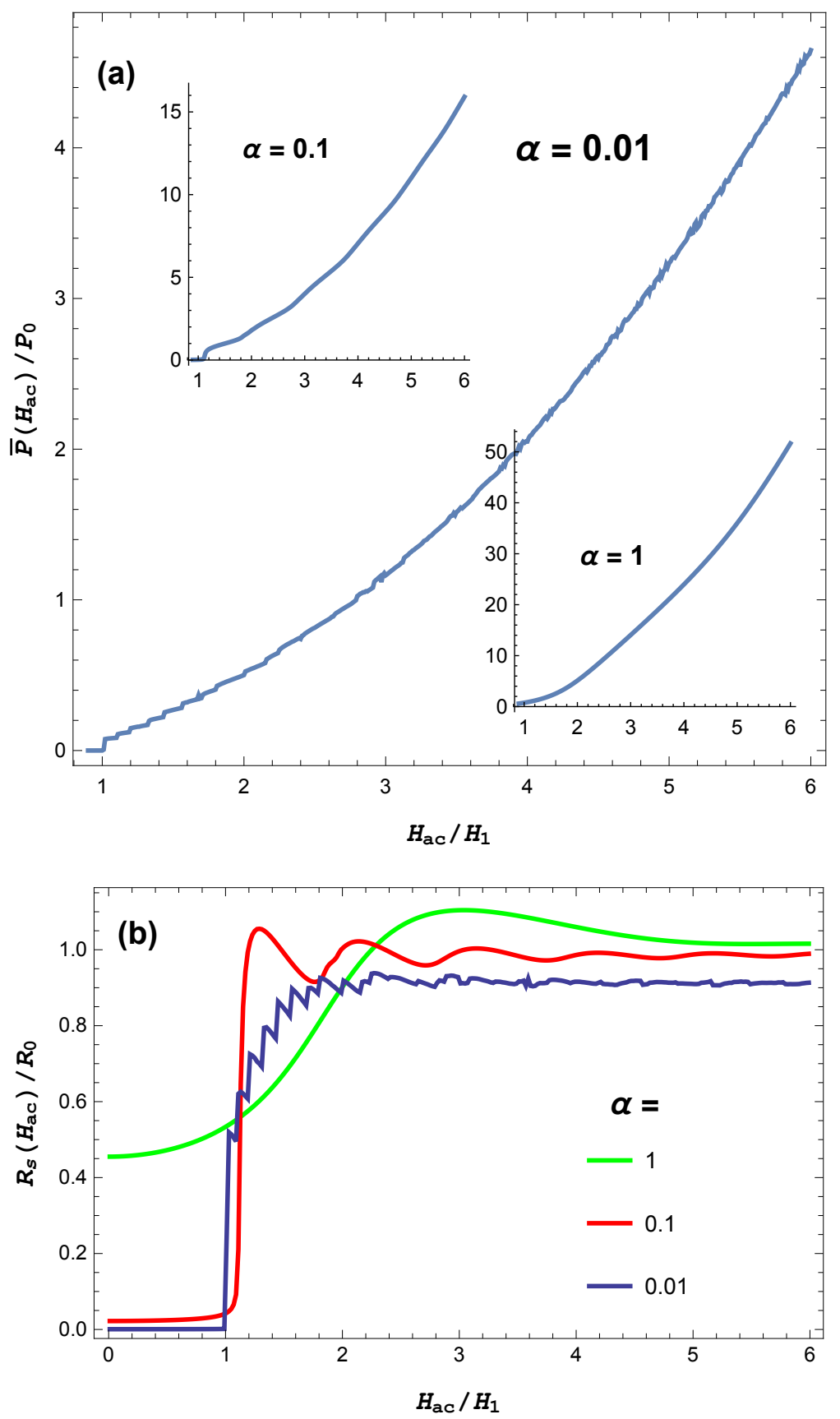

FIG. 30: (a) Plots of $\bar{P}\left(H_{a c}\right)$ for different dimensionless frequencies $\alpha=\omega / \omega_{c}=0.01,0.1$ and 1 , in the overdamped limit. Steps in $\bar{P}\left(H_{a c}\right)$ are associated with the addition of vortices to the junction. (b) The surface resistance $R_{s}\left(H_{a c}\right)$ for different $\alpha$. 


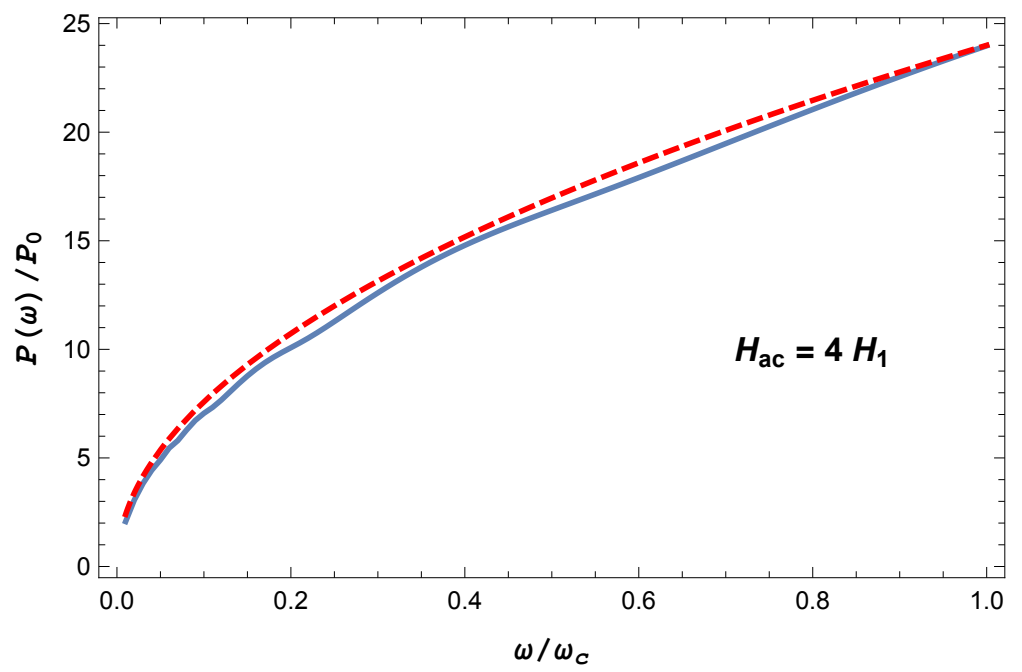

FIG. 31: Plot of $\bar{P}(\omega)$ at $H_{a c}=4 H_{1}$. The dashed line shows a square root function.

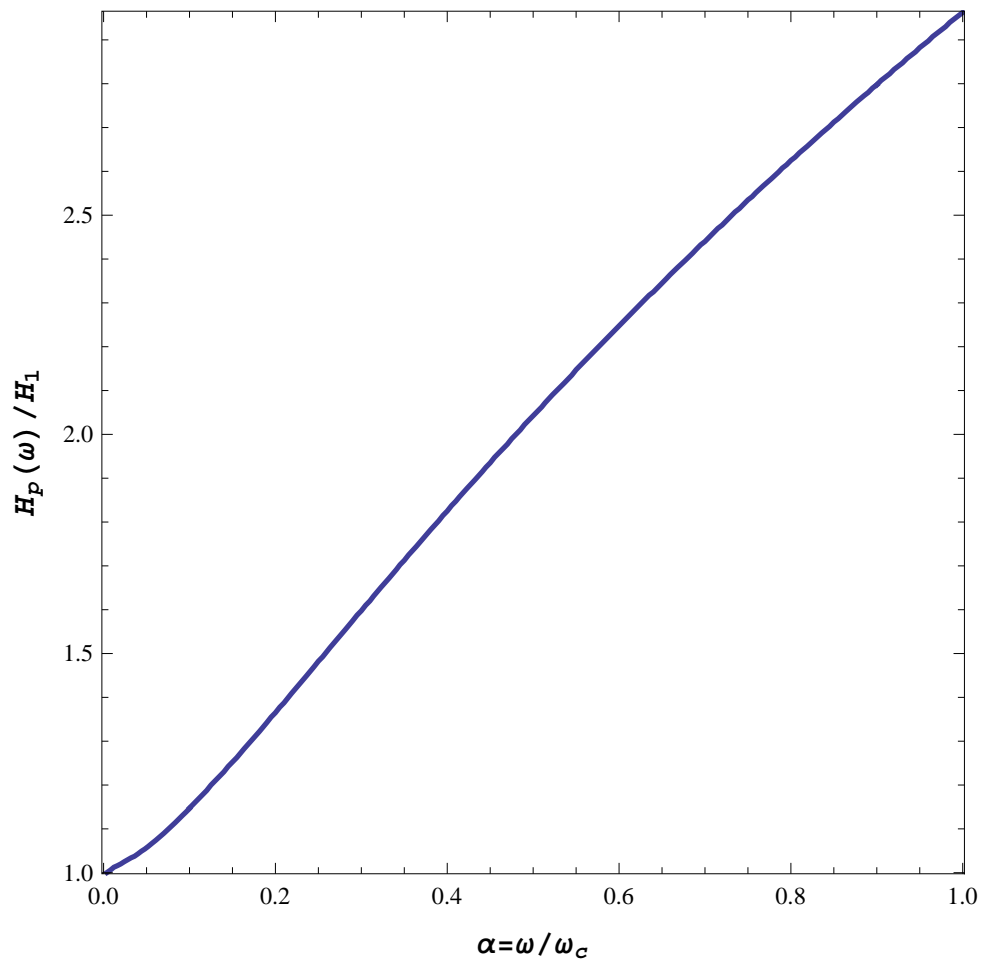

FIG. 32: Frequency dependence of the threshold magnetic field $H_{p}(\omega)$ in the overdamped limit. 


\subsection{ASYMMETRIC AC FIELD}

In the section 3.3 we considered a single mode ac field for which the net Lorentz force averaged over the ac period vanishes. Here we consider two situations in which the net force does not vanish, resulting in a preferential drift velocity of vortices. The first case is a dc magnetic field superimposed onto a single-mode ac field, and the second one is a two-mode ac field with different frequencies. In both cases the ac dynamics of vortices can be tuned by either changing the dc field $H_{d c}$ or the phase shift between the two harmonics.

\subsubsection{AC DRIVEN JUNCTION BIASED WITH A DC FIELD}

Dc magnetic field superimposed onto the ac field can result in interesting effects in LJJ which have many applications for high- $T_{c}$ thin film junctions[70, 79] and flux flow oscillators[70, 71]. As an illustration, Fig. 33 shows the results of calculations in the overdamped limit for $\alpha=0.01, H_{a c}=2 H_{1}$ and different dc field values. The main difference from the results of the section 3.3 is that the positive de field breaks the symmetry between vortices and antivortices, facilitating penetration of vortices and inhibiting penetration of antivortices (and vice versa for negative $H_{d c}$ ). This behavior is clearly seen in Fig. 33. In the limit of $H_{d c} \gg H_{a c}$, the ac field becomes inessential, and flux dynamics approaches the unidirectional flux flow considered in section 3.2.

Similar to the previous sections, we define the dynamic resistance $R_{s}$ using the power balance $\bar{P}=R_{s}\left\langle I^{2}\right\rangle$, where the net current $I=H_{d c}+H_{a c} \sin t$ now contains both dc and ac contributions. Averaging over the ac period gives $\left\langle H_{t o t}^{2}\right\rangle=H_{d c}^{2}+H_{a c}^{2} / 2$, so that

$$
R_{s}=\bar{P} /\left(H_{d c}^{2}+H_{a c}^{2} / 2\right)
$$

Shown in Fig. 34 are the curves $R_{s}\left(H_{a c}\right) / R_{0}$ calculated for $\alpha=0.1$ and different values of $H_{d c}$. One can see that the dc field reduces the field threshold of vortex penetration $H_{p}\left(H_{d c}\right)$ which is now controlled by the maximum instantaneous field value $H_{d c}+H_{a c}$. Thus, we have $H_{p}\left(H_{d c}\right)=H_{1}-H_{d c}$ if $H_{d c}<H_{1}$ and $\omega \ll \omega_{c}$. The resistance at $H_{d c}>H_{1}$ and $H_{a c} \ll H_{d c}$ reduces to the resistance $R_{f}$ for the unidirectional flux flow shown in Fig. 25. We do not consider here a moderately dissipative case $\alpha \beta \sim 1$ for which the resistance $R_{s}$ in superimposed dc and ac fields can become negative [80].

\subsubsection{BI-HARMONIC FIELD AND THE AC RATCHET EFFECT}

It is well-known that a particle driven by an external force in a periodic potential without 


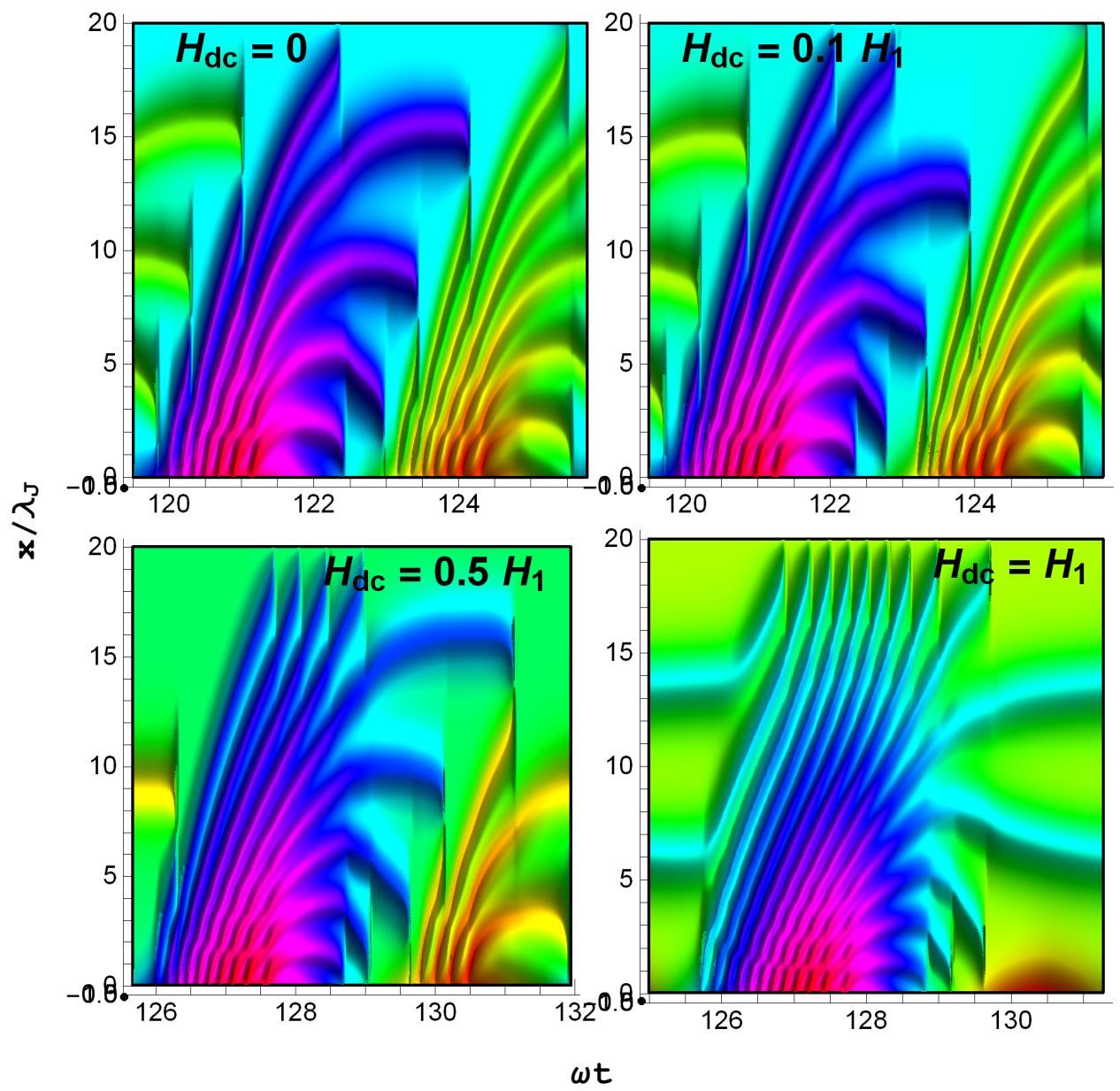

FIG. 33: Evolution of $B(x, t)$ along the junction during the ac period in the overdamped limit calculated for $\alpha=0.01, H_{a c}=2 H_{1}$ and different dc fields specified on the panels. 


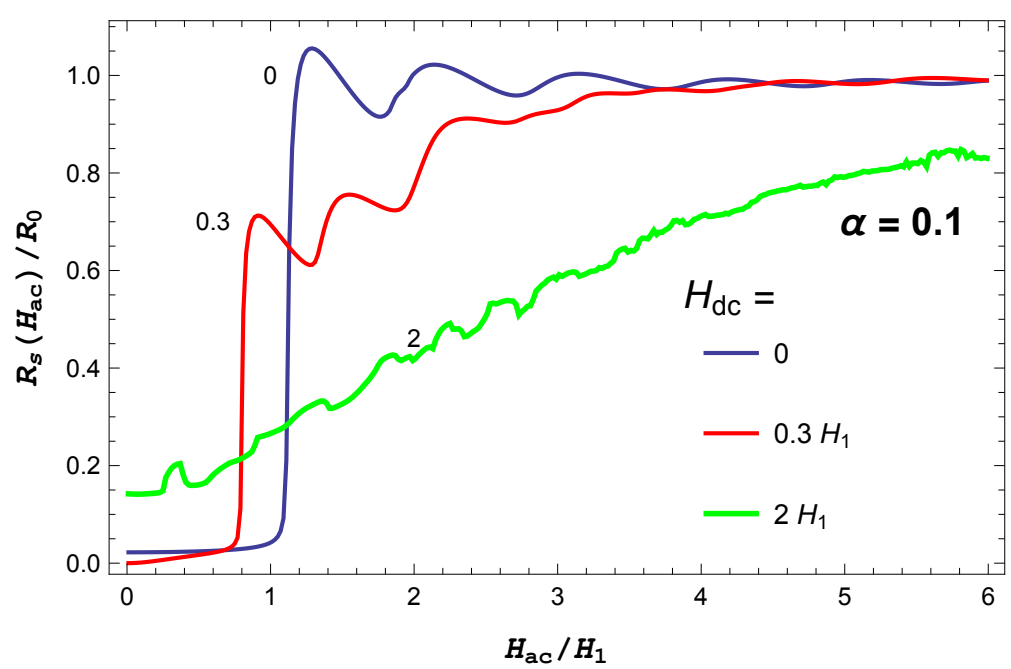

FIG. 34: $R_{s}$ dependence on $H_{a c}$ for a long, finite junction when $\alpha=0.1$ in presence of different values of dc field. When $H_{a c} \rightarrow H_{d c}$ the ac resistance approaches the asymptotic value of $R_{0}$.

reflection symmetry can move with a mean drift velocity $v_{d}$ due to the dc ratchet effect. This situation can occur in a LJJ as well if the Josephson vortex is driven by superimposed dc and ac currents [80] or by a periodic ac force containing more than one harmonics so that $v_{d}$ depends on the phase shift $\vartheta$ between two harmonics with different frequencies $[81,82,83]$. The dc and ac ratchet effects, and the related issues of the Brownian motors [84, 85] have been investigated in biological systems [85], particle separation [85, 86], and vortex motion rectification in superconductors [87, 88, 89, 90].

To see how the dynamic ratchet effect can manifest itself in the LJJ geometry shown in Fig. 19, we consider a Josephson vortex driven by a uniform bi-harmonic current density $J(t)=J_{1} \cos \omega t+J_{2} \cos (2 \omega t+\vartheta)$, where $\vartheta$ is a constant phase shift. We start with a simple model in which the vortex is treated as a particle subject to the ac Lorentz force, so that the velocity of the vortex $v(t)$ is described by the dynamic equation,

$$
M \dot{v}+\left(1+v^{2} / v_{0}^{2}\right) \varsigma v=\Phi_{0} J(t)
$$

where $M$ is the effective vortex mass, $\varsigma$ is the viscous drag coefficient $[42,43]$, and the term $v^{2} / v_{0}^{2}$ describes the first nonlinear correction to the vortex viscosity $[43,62,68]$. We seek the solution of (69) in the form:

$$
v(t)=v_{d}+v_{1} \cos \left(\omega t+\varphi_{1}\right)+v_{2} \cos \left(2 \omega t+2 \varphi_{2}\right)+v_{i}(t)
$$

where $v_{d}$ is a dc drift velocity, and $v_{i}(t)$ is a periodic function which contains higher order 
harmonics. If $v(t) \ll v_{0}$, the nonlinear term in (69) is small, so that $v_{d}$ can be calculated in a perturbation theory [85] by averaging (69) over the ac period:

$$
v_{d}=-\frac{\left\langle v^{3}\right\rangle}{v_{0}^{2}}=-\frac{3 v_{1}^{2} v_{2}}{4 v_{0}^{2}} \cos 2\left(\varphi_{2}-\varphi_{1}\right) .
$$

Here $\left\langle v^{3}\right\rangle$ was calculated from (70) neglecting the higher order harmonics $v_{i}(t)$. In the low-frequency overdamped limit $\omega \ll \varsigma / M$, the mass term in (69) can be neglected and the linearized equations $v(t)=\Phi_{0} J(t) / \varsigma$ for the first and the second harmonics yield $v_{1}=\Phi_{0} J_{1} / \varsigma$ and $v_{2}=\Phi_{0} J_{2} / \varsigma$. Then $(71)$ reduces to:

$$
v_{d}=-\frac{3 \Phi_{0}^{3} J_{1}^{2} J_{2}}{4 v_{0}^{2} \varsigma^{3}} \cos \theta .
$$

This relation shows that the drift velocity can be changed by varying the phase shift $\vartheta$ to make the vortex move either to the left or to the right. The case of two superimposed modes thus appears qualitatively similar to the case of superimposed dc and ac fields considered above because penetration of vortices can be either facilitated or inhibited by varying the phase shift $\vartheta[81,82,83]$. The case of bi-harmonic rf field can model grain boundaries in superconducting resonator cavities in which several resonance electromagnetic modes can be generated [6].

We now solve the overdamped sine-Gordon equation for a bi-harmonic field, $H(t)=$ $H_{a 1} \sin \omega t+H_{a 2} \sin (m \omega t+\vartheta)$ and the boundary conditions,

$$
\theta^{\prime}(0, t)=h_{1} \sin t+h_{2} \sin (m t+\vartheta), \quad \theta^{\prime}(l, t)=0
$$

where $\left\{h_{1}, h_{2}\right\}=\left(2 \pi \mu_{0} d \lambda_{J} / \Phi_{0}\right)\left\{H_{a 1}, H_{a 2}\right\}$ and $m$ is integer.

In the overdamped limit the solutions for $\theta(x, t)$ have the same periodicity as $H(t)$. For even $m$, the ratio of the mean numbers of fluxons and antifluxons can be tuned by varying $\vartheta$, which was observed in Ref. [88, 89]. For instance, as Fig. 35a shows, increasing $\vartheta$ from 0 to $2 \pi / 3$ inhibits penetration of vortices and facilitates penetration of anti-vortices into the junction. For odd values of $m$, the field satisfies the condition $H(t+T / 2)=-H(t)$ so the change of $\vartheta$ does not result in the vortex/antivortex imbalance, although flux dynamics is affected by $\vartheta$. As an example, Fig. 35b shows that varying $\vartheta$ from 0 to $2 \pi / 3$ affects the dynamics of $B(x, t)$ symmetrically for both fluxons and anti-fluxons.

Fig. 36 shows how the dissipated power can be tuned by varying $H_{a 2}$ and $\vartheta$ for the fixed amplitude of the first harmonic, $H_{a 1}$. For $m=2$, the power $\bar{P}$ generally increases with $H_{a 2}$ but the change of $\vartheta$ from 0 to $\pi / 2$ results in humps and dips on the curve of $\bar{P}\left(H_{a 2}\right)$ at 
(a)

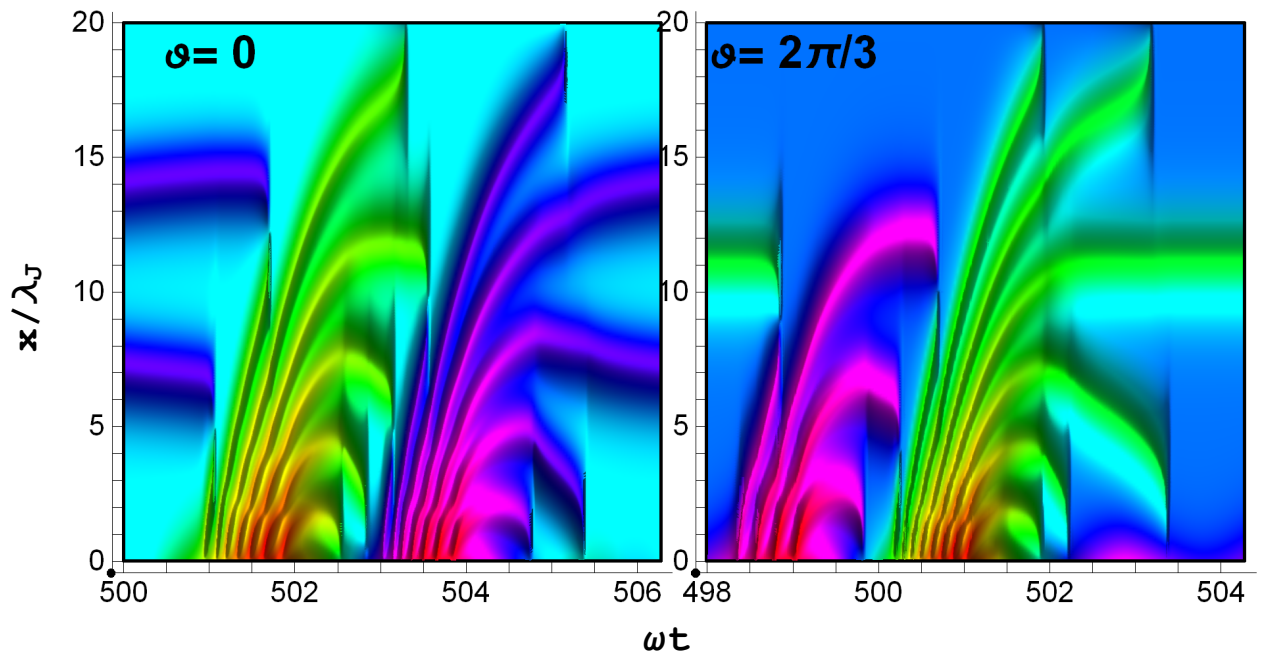

(b)

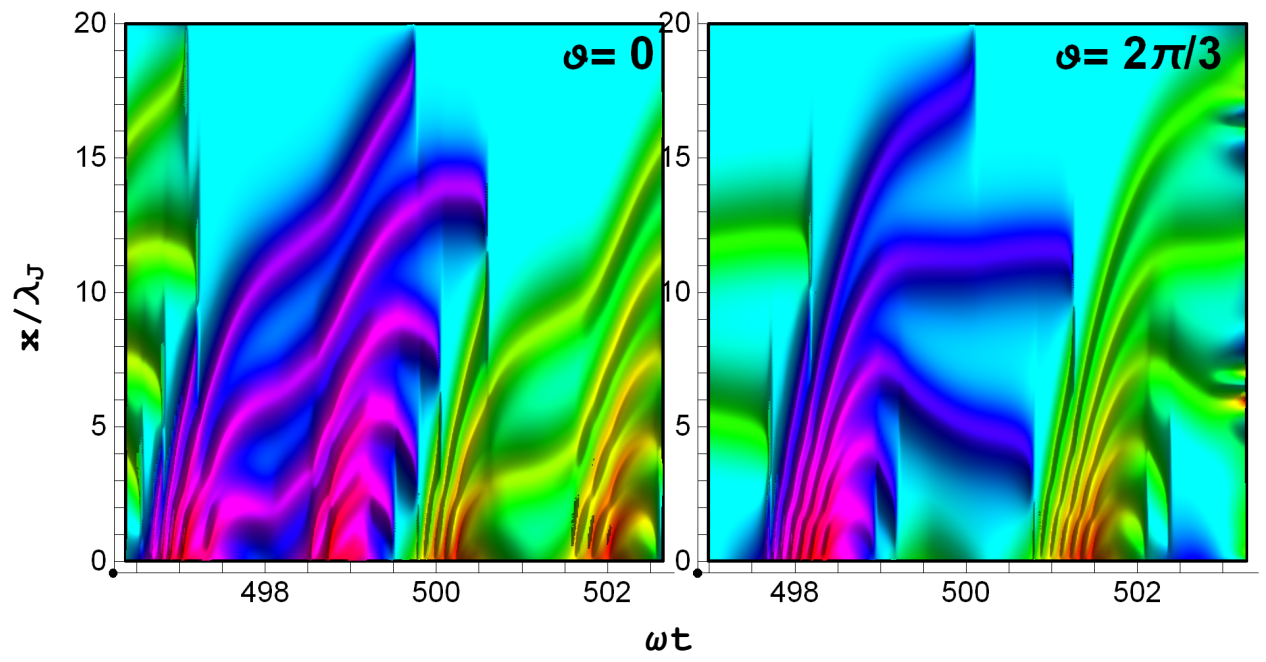

FIG. 35: Evolution of $B(x, t)$ for a $2 \pi$ period of the bi-harmonic magnetic field. (a): $m=2$, $H_{a 2} / H_{a 1}=0.66$ and $\alpha=0.01$. Changing $\vartheta$ from 0 to $2 \pi / 3$ decreases the number of vortices and increases the number of anti-vortices in the junction. (b): $m=3, H_{a 2} / H_{a 1}=0.66$ and $\alpha=0.01$. Varying $\vartheta$ affects flux dynamics but does not result in the vortex/antivortex imbalance. 
$H_{a 2} \approx H_{1} / 2$ and $H_{a 2} \approx 1.8 H_{1}$. For $m=3$, the dips in $\bar{P}\left(H_{a 2}\right)$ are less pronounced but still apparent at $H_{a 2} \approx 0.8 H_{1}$ and $\vartheta=\pi / 2$. In both cases changing $\vartheta$ from 0 to $\pi / 2$ can reduce $\bar{P}\left(H_{a 2}\right)$ in certain regions of $H_{a 2}$ while increasing $\bar{P}\left(H_{a 2}\right)$ in others.

The dependencies of $\bar{P}(\vartheta)$ for $m=2$ and $m=3$, at a fixed field amplitude $\left(H_{a 1}^{2}+\right.$ $\left.H_{a 2}^{2}\right)^{1 / 2}=3 H_{1}$ and different values of $H_{a 2}$ are shown in Fig. 37a and b. Here $\bar{P}(\vartheta)$ can vary rapidly with $\vartheta$, although the maximum change of $\bar{P}(\vartheta)$ does not exceed $10 \%$. Interestingly, the most pronounced reduction of $\bar{P}(\vartheta)$ in the suitable ranges of $\vartheta$ occurs if the amplitude of the second harmonics is small as compared to $H_{a 1}$.

\subsection{SUMMARY AND DISCUSSION}

It has been shown that the electromagnetic response of a long but finite Josephson junctions in ac magnetic fields can be quite complicated due to penetration, oscillation and annihilation of Josephson vortices. The nonlinear dynamics of Josephson vortices results in essential dependencies of the averaged dissipated power $\bar{P}$ and the dynamic resistance $R$ on the field amplitude. Here $\bar{P}(H)$ and $R(H)$ can have steps and peaks due to the change of the number of trapped vortices in the junction as $H$ increases. The calculated field dependence of the surface resistance $R(H)$ is far from linear, inconsistent with the model assumptions of previous works $[17,25]$. It is important to point out that $\bar{P}(H)$ is obtained by averaging the instant power $P(t, H)$ over the ac period during which $P(t)$ has strong spikes due to annihilation of vortices and antivortices in the junction, the magnitude of these power spikes can be much higher than the smooth background contribution to $P(t)$. In high- $J_{c}$ Josephson junctions these power spikes may trigger thermo-magnetic instabilities in the rf field [91].

The penetration of Josephson vortices occurs above the threshold field $H_{p}(\omega)$ at which the dissipated power increases significantly. If the local $J_{c}$ at the edge of the junction is not reduced by materials defects, the dynamic penetration field $H_{p}(\omega)$ remains close to the dc Josephson superheating field of the Meissner state $H_{1}=\Phi_{0} / 2 \pi \mu_{0} \lambda \lambda_{J}$ if $\omega / \omega_{c} \ll 1$. The characteristic frequency $\omega_{c}$ in (43) is proportional to the product $J_{c} R_{j}$, so the frequency dependence of $H_{p}(\omega)$ is most pronounced for low- $J_{c}$ and low resistance junctions. The estimates given above show that for the grain boundaries in $\mathrm{Nb}$, the frequency-dependent correction in $H_{p}(\omega)$ is small for $\omega<\Delta / \hbar$, where $\Delta$ is the superconducting gap.

As was mentioned in the beginning of this chapter, a LJJ of finite length can model the electromagnetic response of grain boundaries in polycrystalline superconductors. The grain boundaries in $\mathrm{Nb}_{3} \mathrm{Sn}$, iron-based superconductors or high- $T_{c}$ cuprates do behave as Josephson weak links $[2,11]$, so one can expect that the nonlinear effects addressed here, 

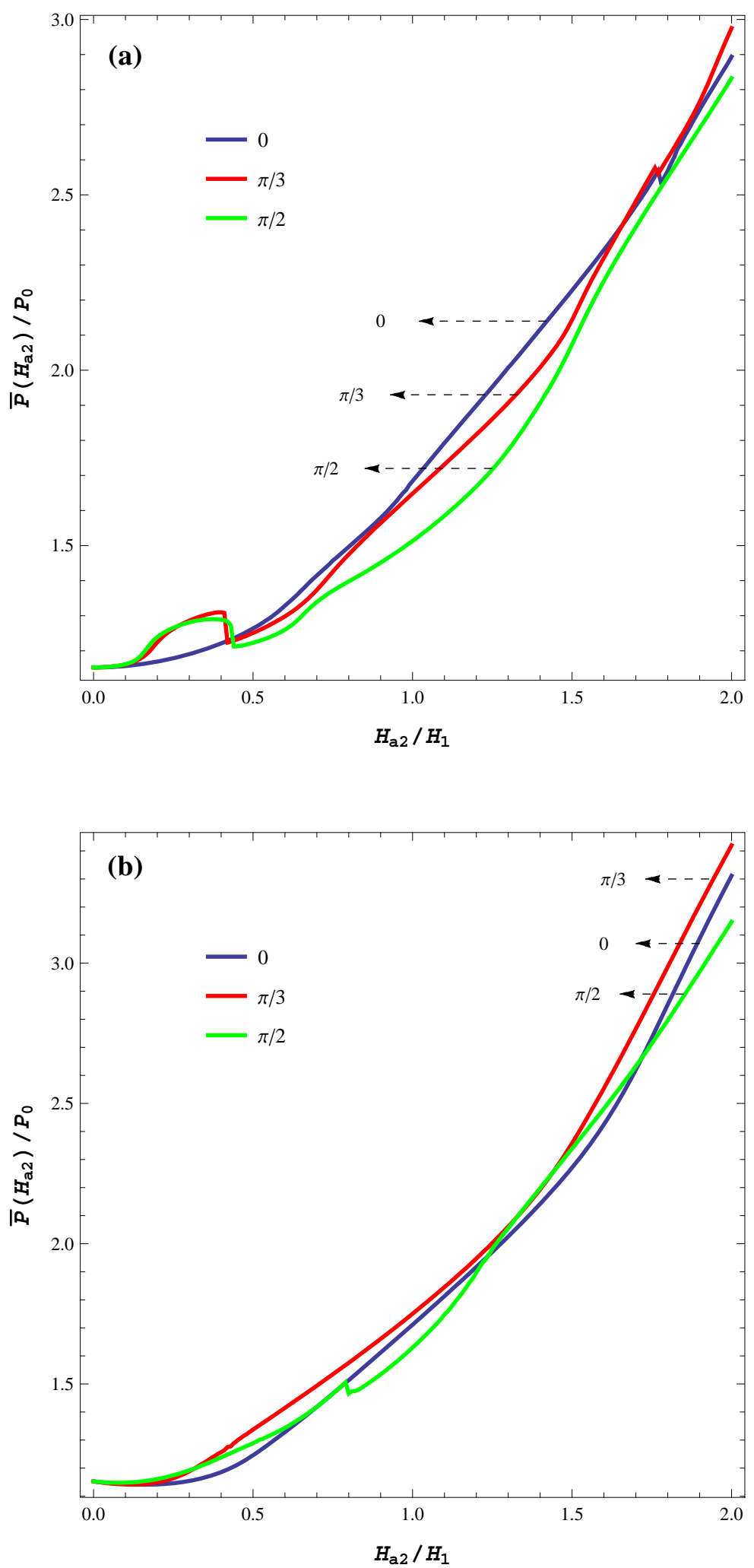

FIG. 36: Plots of the average dissipated power $\bar{P}$ as a function of second mode amplitude $H_{a 2}$, for different values of relative phase $\vartheta$ while $\alpha=0.05$ and the fundamental mode is kept constant at $H_{a 1}=2 H_{1}$. (a) $m=2$. (b) $m=3$. 

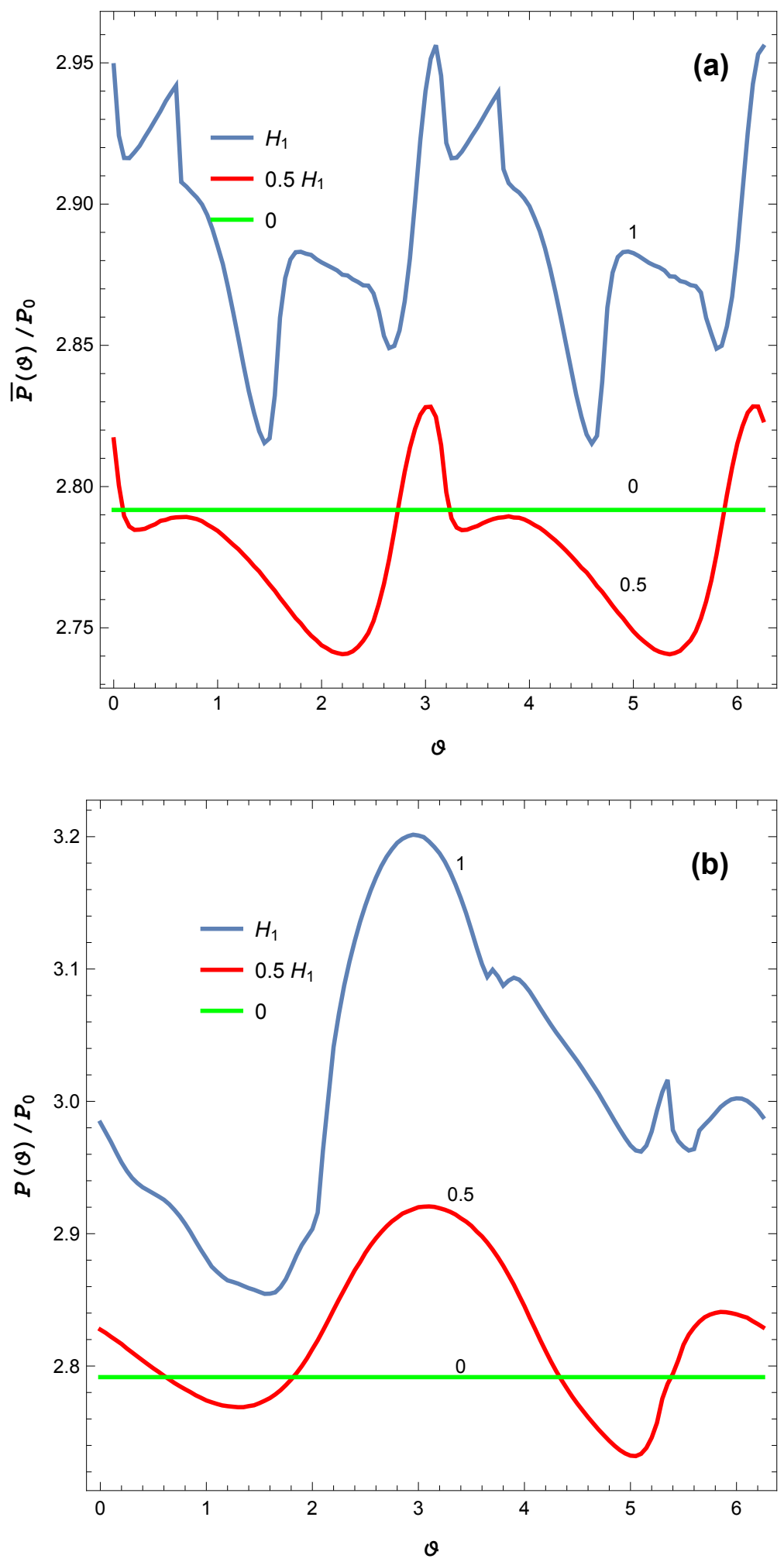

FIG. 37: Plots of the average power $\bar{P}$, as a function of the relative phase $\vartheta$, for $\alpha=0.05$, fixed total field magnitude $\left(H_{a 1}^{2}+H_{a 2}^{2}\right)^{1 / 2}=3 H_{1}$ and different values of $H_{a 2}$ : (a) $m=2$ and (b) $m=3$. The solid horizontal line at $\approx 2.79$ in both plots shows $\bar{P}$ at $H_{a 1}=3 H_{1}$ and $H_{a 2}=0$. 
can manifest themselves in the surface impedance at rather low fields $H \simeq H_{1}<H_{c 1}$. Such effects can also be essential for the rf performance of polycrystalline multilayer screens which were suggested to enhance the breakdown field of $\mathrm{Nb}$ resonator cavities [13]. By contrast, the grain boundaries in $\mathrm{Nb}$ resonator cavities appear to be strongly coupled and do not behave as the conventional Josephson junctions up to very high magnetic fields at which densities of screening current become of the order of the depairing current density. As a result, the field onset of penetration of mixed Abrikosov-Josephson vortices $[76,77,78]$ is close to the lower critical field of intra-grain vortices $H_{c 1} \simeq 170 \mathrm{mT}$. The dynamics of these strong-link GBs is the subject of the next two chapters and the governing equations drastically differ from sine-Gordon equation in (43). 


\section{CHAPTER 4}

\section{VORTEX DYNAMICS IN STRONGLY-COUPLED GRAIN BOUNDARIES AND CHERENKOV INSTABILITY}

Sine-Gordon equation in (39) was obtained based on the assumption that the magnetic field $B(x, t)$ produced by vortex currents in the junction and the phase difference $\theta(x, t)$ both vary slowly over the same length scale $\mathcal{L} \gg \lambda$. In other words, the magnetic field in a point $x$ inside the junction is determined by the value of the $\frac{\partial}{\partial x} \theta(x, t)$ at the same point using (35). However, there are numerous conditions where this fundamental assumption is violated, hence a more rigorous treatment of the electrodynamics of Josephson junctions is required. In the following we will outline some of the possible scenarios where the local relation between phase and field breaks down and (35) is no longer applicable.

\subsection{MECHANISMS OF NONLOCALITY}

A direct result of the Lorentz-invariant sine-Gordon equation is the contraction of a moving Josephson vortex in underdamped junctions. Therefore, at high velocities the contraction is such that the size of the vortex becomes comparable to penetration $\operatorname{depth}, \mathcal{L}(v) \sim \lambda$, hence a nonlocal version of sine-Gordon equation is required.

On the other hand, in sufficiently narrow junctions where $W \leq \lambda$, the stray magnetic fields outside the junction generate additional surface currents which have to be taken into account. In this case, the characteristic space scale for the field $B(x, t)$ is determined by the Pearl length[92]

$$
\lambda_{P}=\frac{2 \lambda^{2}}{W}
$$

which can be much larger than the London penetration depth $\lambda$. In the static case $\mathcal{L}$ is of the order of $\lambda_{J}$, the Josephson length. Therefore, if the condition $\lambda_{P} \geq \lambda_{J}$ holds, the stray magnetic field becomes important and results in a nonlocal equation.

An intrinsic emergence of nonlocality happens when the energy of magnetic field inside the superconducting electrodes of the junction becomes considerable such that the penetration depth is of the order of, or larger than the spatial scale of variations of the phase difference i.e. $\lambda \geq \lambda_{J}$. For instance, the copper-oxide high- $\mathrm{T}_{c}$ superconductors contain coherent planar 
defects such as twins, stacking faults, low-angle grain boundaries which do not cause strong crystalline lattice distortions, but can lead to local reduction of superconducting gap $\Delta$ due to small value of $\xi$. These defects can be treated as intrinsic Josephson junctions with high values of $J_{c}$, hence small values of Josephson penetration depth $\lambda_{J}$. In terms of the critical current density, the nonlocality emerges when $J_{l}<J_{c}<J_{d}$ where $J_{d}$ is the depairing current density and $J_{l}=J_{d} / \kappa$ is given by the condition $\lambda_{J}\left(J_{l}\right)=\lambda$. This condition applies to a wide range of currents in extreme type-II superconductors where $\kappa \gg 1$. An analogous situation may occur in conventional low- $\mathrm{T}_{c}$ superconductors as well, for example, in optimized high- $J_{c}$ $\mathrm{Nb}$-Ti alloys, where the strong pinning is caused by a dense network of thin $\alpha$-Ti ribbons, or in $\mathrm{Nb}_{3} \mathrm{Sn}$ where the pinning is due to grain boundaries. In high performance SRF applications where the highest achievable magnetic field is desired, strong-linked GBs are required in order to delay the penetration of vortices to higher magnetic fields. The nonlocal range of currents in $\mathrm{Nb}_{3} \mathrm{Sn}$ as a promising $\mathrm{SRF}$ material describes the important field range of $H_{c} / \kappa<H<H_{c}$ where $\kappa \approx 20$ and $H_{c} \approx 540 \mathrm{mT}$.

\subsection{ELECTRODYNAMICS OF NONLOCAL JUNCTIONS}

In either cases of the above, the equation which generalizes $(44)$ to $\theta(x, t)$ and $B(x, t)$ varying over any lengths larger than the superconducting coherence length $\xi$, namely, $l_{J}$ and $\lambda \gg \xi$, is given by $[76,77,78,93,94,95,96,97,98,99,100,101,102,103,104]$

$$
\ddot{\theta}+\eta \dot{\theta}=l_{J} \int_{-\infty}^{\infty} G(x, u) \theta^{\prime \prime}(u, t) d u-\sin \theta+\beta
$$

where $l_{J}=\lambda_{J}^{2} / \lambda, x$ and $u$ are in normal units and the nonlocality kernel $G(x, u)$ depends on the sample geometry and is often of the convolution form, i.e.

$$
G(x, u)=G(x-u)
$$

It is worth mentioning that the local description in (44) arises in the limit case of $G(x, u)=$ $\lambda \delta(x-u)$ where $\delta(x)$ is the Dirac delta function.

The nonlocal models can be divided into two basic groups; first, those treating the internal nonlocality in bulk junctions[76, 96, 101]. For instance, in the simplest case of planar junction in a bulk superconductor the kernel function is given by[76]

$$
G(x-u)=\pi^{-1} K_{0}\left(\frac{|x-u|}{\lambda}\right)
$$

where $K_{0}(x)$ is the zeroth order modified Bessel function which has a logarithmic singularity at $x=0$ and decays exponentially at large distances, $|x|>\lambda$. The second group are those 
dealing with nonlocal effects due to external stray fields resulting from the geometry of the junction and its electrodes $[93,95,97,99,100]$. For an edge junction in a thin film of thickness $W \ll \lambda$, we have

$$
G(x-u)=\frac{1}{2}\left[\mathbf{H}_{\mathbf{0}}\left(\frac{|x-u|}{\lambda_{P}}\right)-Y_{0}\left(\frac{|x-u|}{\lambda_{P}}\right)\right]
$$

where $\lambda_{P}=2 \lambda^{2} / W$ and $\mathbf{H}_{\mathbf{0}}(x)$ and $Y_{0}(x)$ are Struve and Bessel functions, respectively, with same logarithmic singularity at $x=0$.

\subsubsection{MIXED ABRIKOSOV-JOSEPHSON VORTEX}

In the limit of strong nonlocality (bulk: $\lambda_{J} \ll \lambda$, thin film: $\lambda_{J} \ll \lambda_{P}$ ), the expansion of the kernel $G(x, u)$ in (77) and (78) for small arguments reveals that only the universal logarithmic part of $G(x, u)$

$$
G_{0}(|x-u|)=\frac{1}{\pi} \ln \left(\frac{1}{|x-u|}\right)
$$

is essential while the non-singular geometry dependent part can be disregarded. Replacing $G(x, u)$ with this logarithmic kernel and performing integration by parts, (75) reduces to

$$
\ddot{\theta}+\eta \dot{\theta}=\frac{l_{J}}{\pi} \int_{-\infty}^{\infty} \frac{d u}{u-x} \frac{\partial \theta}{\partial u}-\sin \theta+\beta
$$

which describes a mixed Abrikosov-Josephson (AJ) vortex with Josephson core of length $l_{J}=\lambda_{J}^{2} / \lambda \approx \xi J_{d} / J_{c}$ along the junction[94]. Such AJ vortices in which the order parameter in the core is not suppressed have been revealed by transport measurements on low-angle grain boundaries in cuprates [105, 106, 107], annular Josephson junctions [108, 109, 110, 111], magnetization of thin films [112], and STM imaging of step edge junctions in $\mathrm{Pb}$ and In monolayers on Si substrates $[113,114,115]$. Equation $(80)$ in the overdamped limit of $\eta \gg 1$ has an exact solution

$$
\theta(x, t)=\pi+\sin \beta+2 \tan ^{-1}\left(\frac{x-v t}{\mathcal{L}}\right)
$$

that describes a driven AJ vortex core with weak suppression of $\Delta(x)$ and the length and velocity given by

$$
\mathcal{L}=\frac{l_{J}}{\sqrt{1-\beta^{2}}}, \quad v=-\frac{\beta l_{J}}{\tau}
$$

where $\tau=\eta / \omega_{J}$ and the vortex expands as $\beta$ increases[94].

\subsection{PINNING OF J AND AJ VORTICES}


It has been shown that the most effective core pinning of vortices is by normal precipitates where the maximum pinning force in the case of optimal size of pinning centers is $f \sim$ $\gamma\left(\Phi_{0} / 4 \pi \lambda\right)^{2} / \mathcal{L}$ which is inversely proportional to the size of the vortex ( $\gamma$ being the fraction of volume occupied by pinning centers)[76]. Therefore, an Abrikosov vortex due to its small normal core size of $\sim \xi$ is strongly pinned while a Josephson vortex with size $\lambda_{J} \gg \xi$ is very weakly pinned in defects. Considering that the size of the AJ vortex is a crossover between the Abrikosov and Josephson $\mathcal{L}>\xi$, it can qualitatively be inferred that AJ vortices are weakly pinned by defects compared to Abrikosov vortices and can easily be depinned by currents even less than the intra-grain critical current.

\subsection{CHERENKOV RADIATION OF MOVING VORTEX}

Unlike (44), the general (75) at $\eta=0$ is not Lorentz-invariant, so a uniformly moving vortex can radiate Cherenkov waves $\delta \theta(x, t) \propto \exp \left(i k x-i \omega_{k} t\right)$ with the phase velocities $\omega_{k} / k$ smaller than $v[96,104]$. Setting $\theta(x, t)=\theta_{\infty}+\delta \theta(x, t)$ where $\sin \theta_{\infty}=\beta$, and linearizing (75) with respect to small disturbances $\delta \theta(x, t)$ for a uniform dc current and $\eta=0$, yields the dispersion relation $\omega_{k}^{2}=\left[\cos \theta_{\infty}+l_{J} k^{2} G(k)\right] \omega_{J}^{2}$. Thus, the condition of Cherenkov radiation $k v>\omega_{k}$ is given by:

$$
k v>\omega_{J}\left[\sqrt{1-\beta^{2}}+l_{J} k^{2} G(k)\right]^{1 / 2},
$$

where $G(k)$ is the Fourier image of $G(x)$. Here $G(k)$ decreases as $1 / k$ at $k>\Lambda^{-1}(\Lambda$ being the magnetic penetration depth) so (83) is satisfied if $k>k_{c}$, where the maximum wavelength $\mathrm{E}_{c}=2 \pi / k_{c}$ increases with $v$. For a bulk junction, we have $G(k)=\lambda / \sqrt{1+\lambda^{2} k^{2}}$, so the threshold $k_{c}$ at which (83) becomes equality can be evaluated in the limit of $\lambda / \lambda_{J} \ll 1$ by expanding $\left(1+\lambda^{2} k_{c}^{2}\right)^{-1 / 2} \approx 1-\lambda^{2} k_{c}^{2} / 2$ and solving the resulting bi-quadratic equation for $k_{c}$ :

$$
k_{c}^{2} \lambda^{2}=1-\frac{v^{2}}{c_{s}^{2}}+\left[\left(1-\frac{v^{2}}{c_{s}^{2}}\right)^{2}+\frac{2 \lambda^{2}}{\lambda_{J}^{2}} \sqrt{1-\beta^{2}}\right]^{1 / 2} .
$$

The maximum Cherenkov wavelength $\mathrm{E}_{c}=2 \pi / k_{c}$ thus increases as $\beta$ and $v$ increase, approaching

$$
\mathrm{E}_{c} \rightarrow \frac{2^{3 / 4} \pi \sqrt{\lambda \lambda_{J}}}{\left(1-\beta^{2}\right)^{1 / 8}}, \quad v \rightarrow c_{s}
$$

hence $k_{c}^{2} \lambda^{2} \ll 1$, which justifies the above expansion of $G(k)$ in small $k_{c}$ at $\lambda / \lambda_{J} \ll 1$. For instance, at the vortex instability threshold $\beta=\beta_{s} \approx 0.67$ calculated for $\lambda_{J} / \lambda=10$ in a bulk junction (section 4.5.1), (85) gives $\mathrm{E}_{c} \approx 19 \lambda=1.9 \lambda_{J}[116]$. 


\subsection{VORTEX DYNAMICS IN DIFFERENT NONLOCAL GEOMETRIES}

In general solutions of (75) for different geometries can only be obtained numerically. In the following we will present the result of simulation of this equation for a traveling vortex moving with constant velocity in different geometries of infinitely long bulk, thin film edge and overlap junctions shown in Fig. 38.

\subsubsection{BULK JUNCTION}

For a bulk junction shown in Fig. 38(a), (75) becomes [76]

$$
\ddot{\theta}+\eta \dot{\theta}=\frac{\lambda_{J}}{\pi \lambda} \int_{-\infty}^{\infty} K_{0}\left(\frac{\lambda_{J}|x-u|}{\lambda}\right) \frac{\partial^{2} \theta}{\partial u^{2}} d u-\sin \theta+\beta,
$$

where spatial coordinates $x$ and $u$ are normalized in units of $\lambda_{J}$. For conventional Josephson weak links with $\lambda_{J} \gg \lambda$, the solution $\theta(x, t)$ varies slowly over the sharply-peaked $K_{0}\left[\lambda_{J}(x-\right.$ $u) / \lambda]$ which thus can be replaced with $\pi \lambda \delta(x-u) / \lambda_{J}$, so that (86) reduces to the sine-Gordon equation in (44). We solved (86) for two cases: a relatively low- $J_{c}$ Josephson junction with $\lambda_{J} / \lambda=10$ which is usually described by the sine-Gordon equation in (44), and a nonlocal high- $J_{c}$ junction with $\lambda / \lambda_{J}=\sqrt{10}$.

Shown in Figs. 39 and 40 are the numerical results for a planar bulk junction at $\eta=0.05$ and the large ratio $\lambda_{J} / \lambda=10$. Here the nonloal equation in (86) reveals the effects which are not captured by (44), particularly a trailing tail of Cherenkov radiation behind a vortex moving with a constant velocity [96]. As plotted, with the increase in driving current the vortex velocity increases, hence the amplitude and the wavelength of radiation increase. Our simulaiton shows that with further increase in current, the vortex becomes unstable at $\beta>\beta_{s}$, the instability is triggered at the highest maximum of Cherenkov wave where $\theta_{m}$ reaches a critical value $\theta_{c} \approx 8.65-8.84$, depending on $\eta$ and $\lambda / \lambda_{J}$. Here $\theta_{c}$ is confined within the interval $5 \pi / 2<\theta_{c}<3 \pi$ in which a uniform state of a Josephson junction is unstable $[42,43]$. As the velocity increases, the domain where $5 \pi / 2<\theta(x-v t)<3 \pi$ behind the moving vortex widens and eventually becomes unstable as its length exceeds a critical value. This suggests a qualitative picture of the vortex instability caused by the appearance of a trailing critical nucleus being in the unstable $\pi$-junction state $[42,43]$ caused by strong Cherenkov radiation. The latter appears entirely due to the Josephson nonlocality described by (86), which has no steady-state vortex solutions at $J>J_{s}$ where $J_{s}$ can be well below $J_{c}$ at which the whole junction switches into a resistive state[116].

The dynamic solutions of (86) at $\beta>\beta_{s}$ change strikingly. Our simulations have shown 

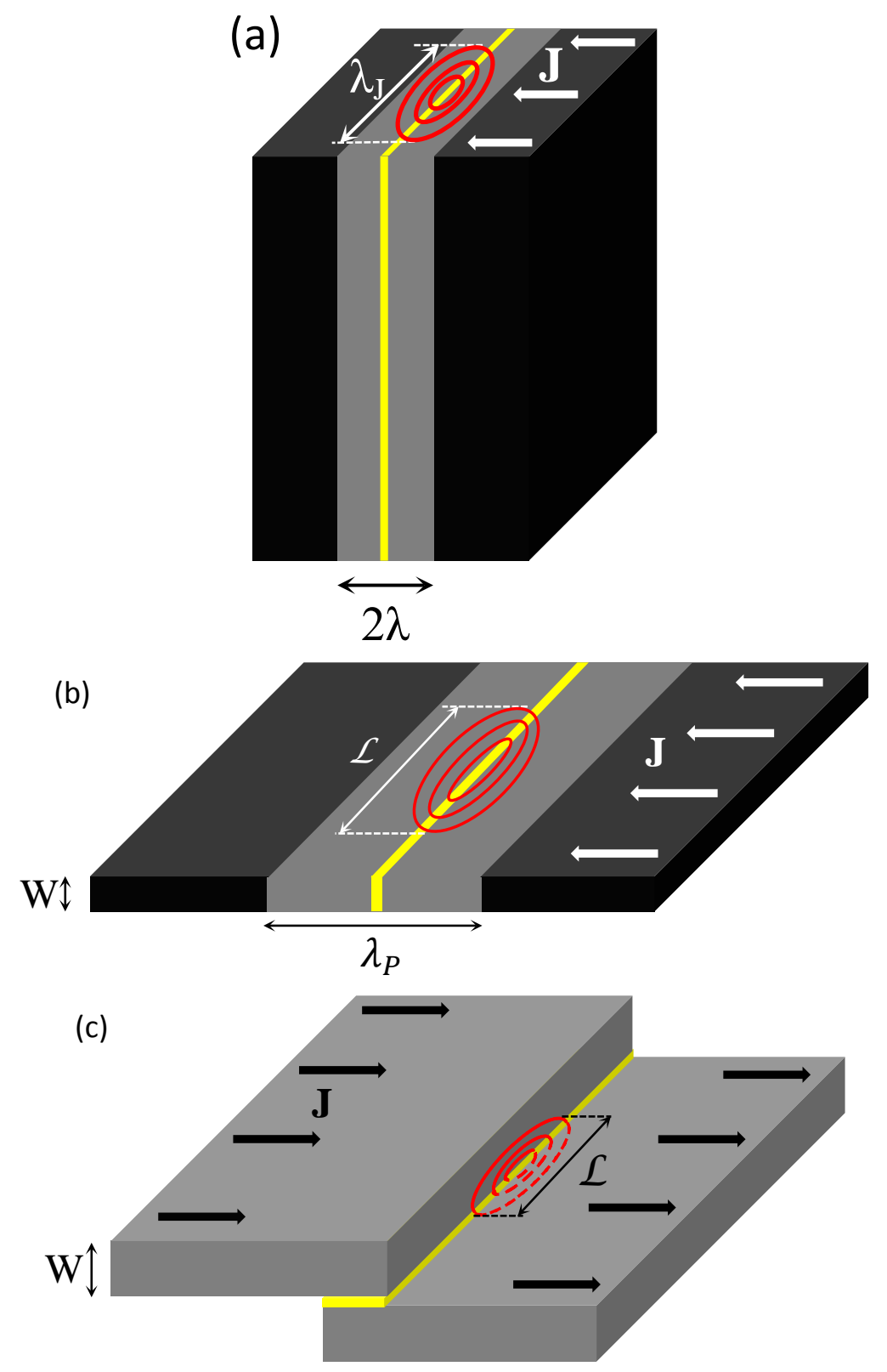

FIG. 38: Geometries of long Josephson junctions for which calculations were performed. (a) bulk junction, (b) thin film edge junction, (c) thin film overlap junction. The yellow line shows the Josephson contact, and gray depicts regions of circulating screening currents in the direction perpendicular to the contact. Current streamlines in a vortex are shown by red. 


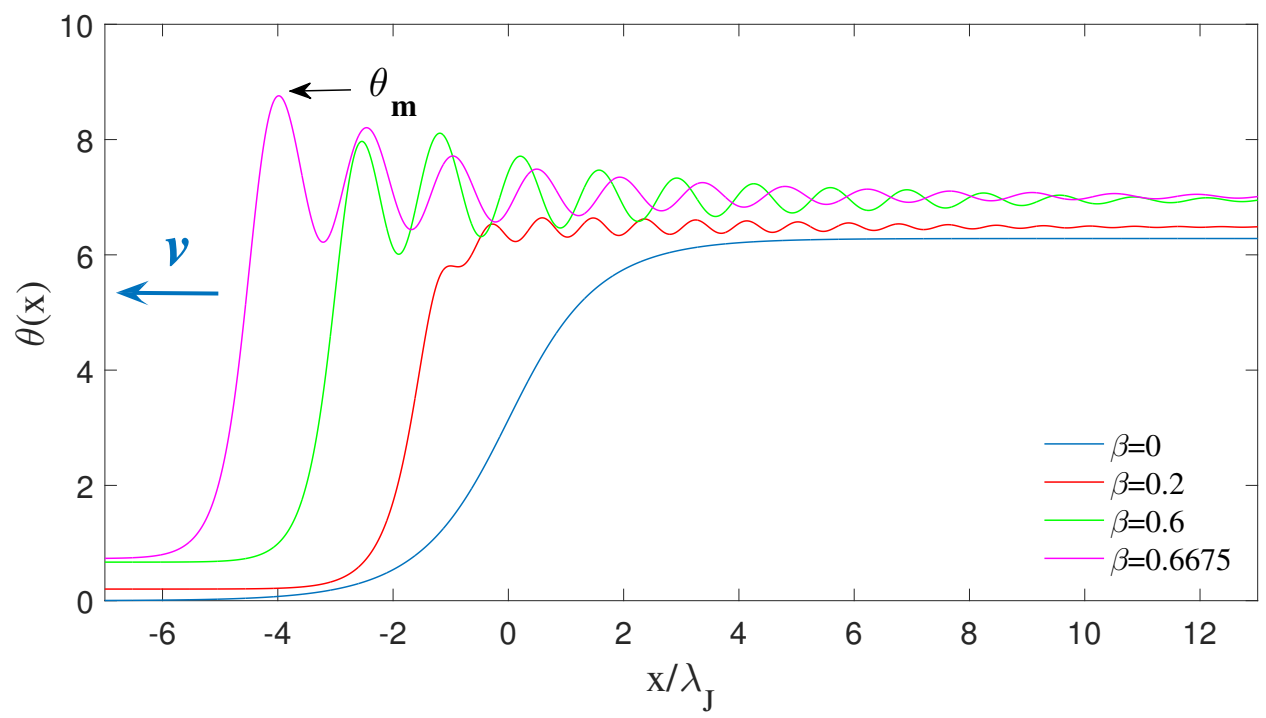

FIG. 39: A sequence of steady state phase profiles in a propagating vortex (shifted horizontally for clarity in the moving frames) calculated for a bulk junction by solving (86) with $\eta=0.05, \lambda_{J} / \lambda=10$ and for different values of $\beta$. At $\beta_{s}=0.6676$ the peak amplitude of Cherenkov wave reaches $\theta_{c}=8.76$ and starts growing and evolving into an expanding vortex-antivortex pair.

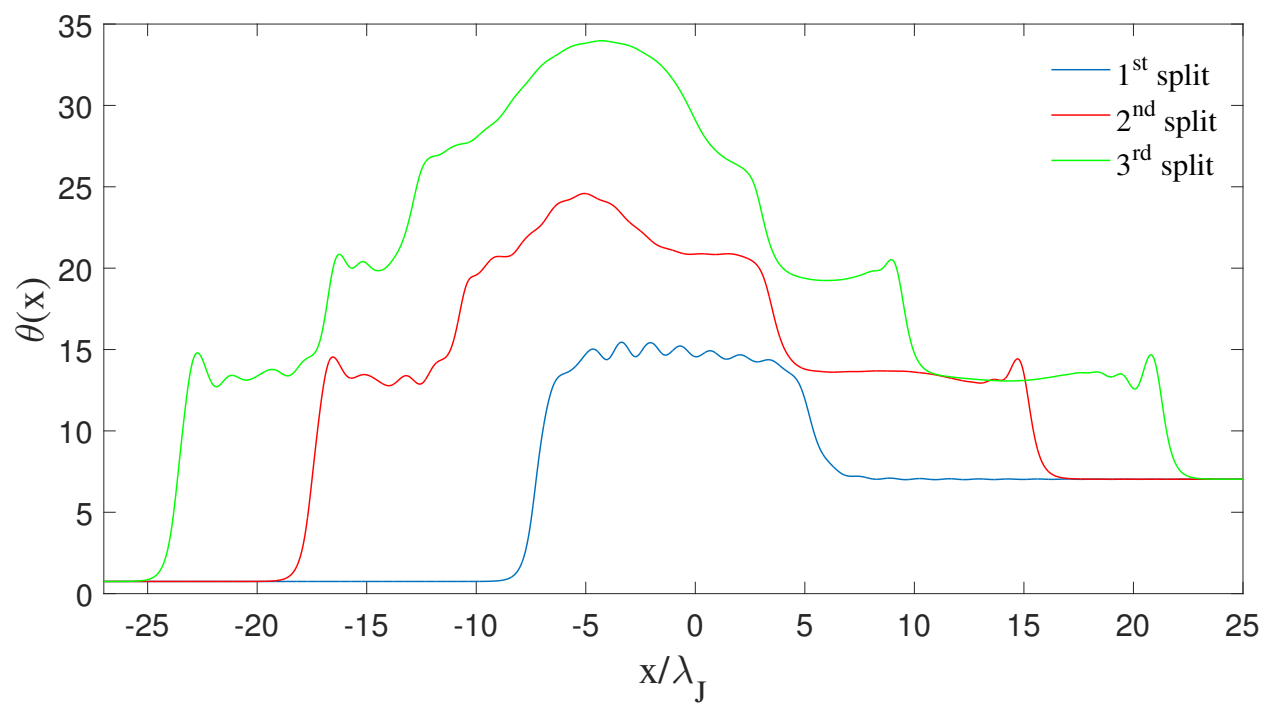

FIG. 40: Snapshots of $\theta(x)$ at $\beta=\beta_{s}=0.6676$ in a bulk junction of $\eta=0.05$ and $\lambda_{J} / \lambda=$ 10 which show first three dissociations of the unstable vortex into vortex-antivortex pairs accompanied by Cherenkov radiation. Movies showing the initial stage of vortex instability and formation of the phase pile after multiple generations of vortex-antivortex pairs are available in Ref. [140]. Notice that $\theta(\infty)-\theta(-\infty)=2 \pi$ remains fixed by the phase difference in the initial vortex. 
that the instability originates at the highest maximum $\theta=\theta_{m}$ of the trailing Cherenkov wave which starts growing and eventually turning into an expanding vortex-antivortex pair, as shown in Fig. 40. As the size of this pair grows, it generates enough Cherenkov radiation to produce two more vortex-antivortex pairs which in turn produce new pairs. Continuous generation of vortex-antivortex pairs results in an expanding dissipative domain in which vortices accumulate at the left side, antivortices accumulate at the right side, while dissociated vortices and antivortices pass through each other in the middle. As a result, $\theta(x, t)$ evolves into a growing "phase pile" with the maximum $\theta_{m}(t)$ increasing approximately linear with time and the edges propagating with a speed which can be both smaller and larger than $c_{s}$, the phase difference $\theta(\infty)-\theta(-\infty)=2 \pi$ between the edges remains fixed. Figure 41 shows the 3D image of the initial stage of dynamic separation of vortices and antivortices. Here the local magnetic field $B(x, t)$ oscillates strongly at the moving domain edges but becomes rather smooth away from them, as shown in Fig. 42. In the most part of the phase pile overlapping vortices are indistinguishable, yet the net flux $\Phi=\Phi_{0}$ of this evolving multiquanta magnetic dipole remains quantized.

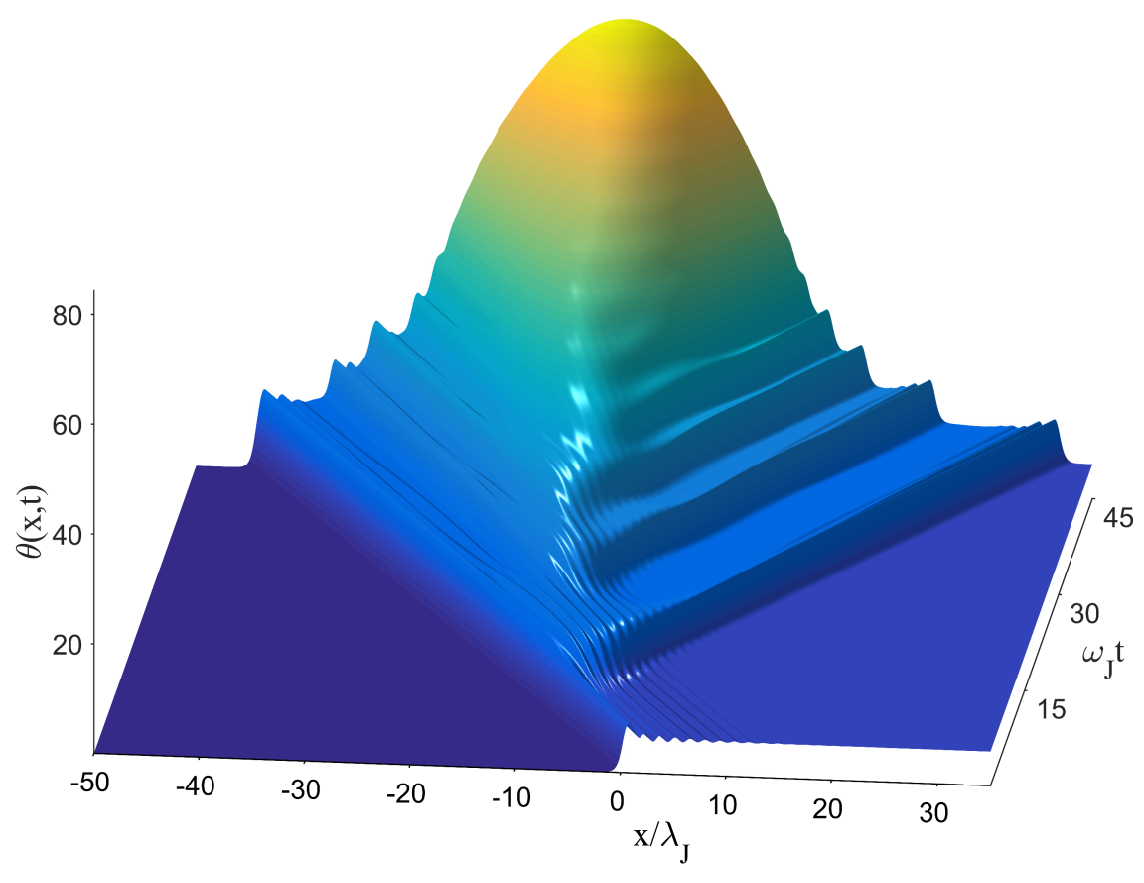

FIG. 41: A 3D image of the evolution of phase pile triggered by an unstable vortex. The dynamic phase distribution $\theta(x, t)$ was calculated from (86) for a bulk junction at $\beta=0.6676$, $\lambda_{J} / \lambda=10$ and $\eta=0.05$. Here the maximum phase $\theta_{m}(t)$ increases approximately linear with time while the edge vortices move with constant velocities close to $c_{s}$. Individual vortices and antivortices clearly visible at the edges of the expanding phase pile overlap strongly toward its central part. 


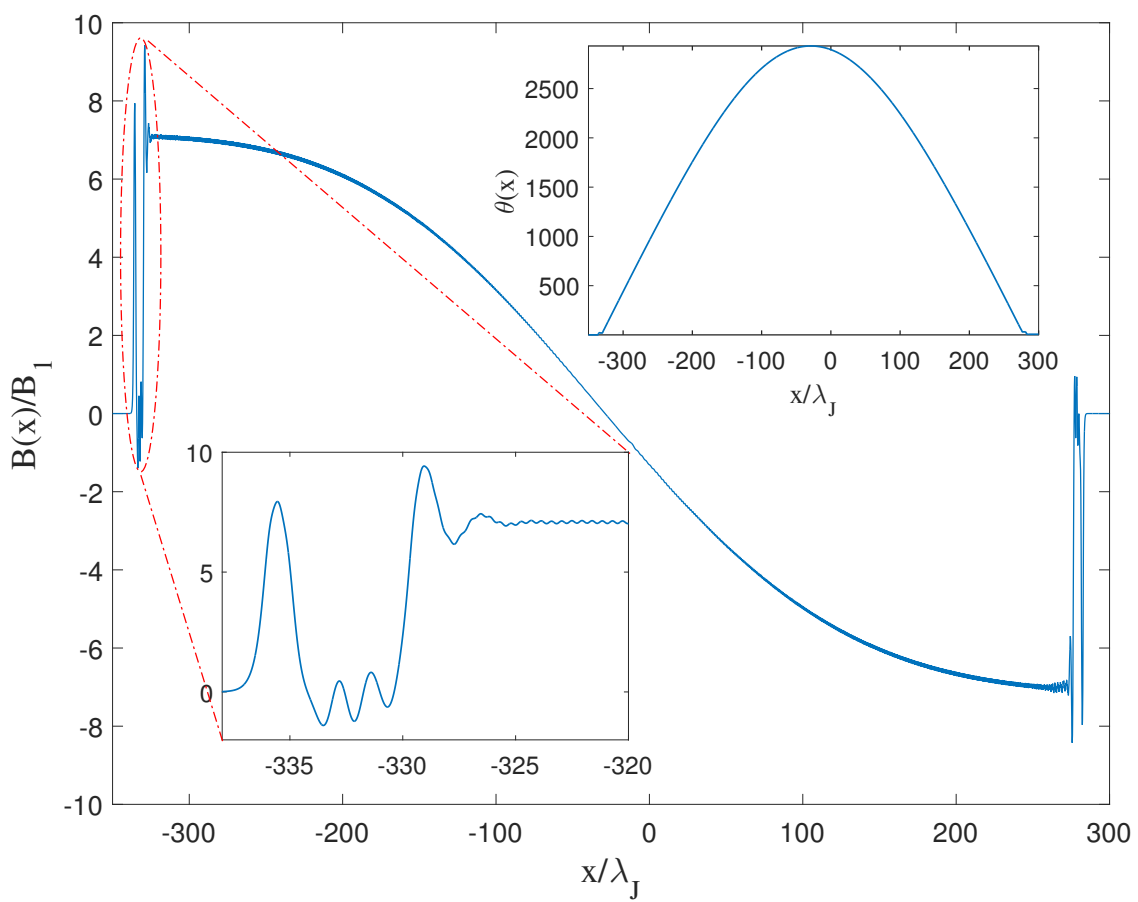

FIG. 42: A snapshot of the normalized magnetic field $B(x, t) / B_{1}$. Here $B(x, t)$ was calculated from (86) for a bulk junction at $\eta=0.05, \beta=0.6676, \lambda_{J} / \lambda=10$ and $B_{1}=\Phi_{0} / 2 \pi \lambda \lambda_{J}$. Inset shows the corresponding phase distribution, $\theta(x, t)$. One can clearly see a complex structure of the left leading edge comprised of a vortex overlapping with a vortex-antivortex pair. Away from the edges vortices overlap so strongly that the Cherenkov radiation gets suppressed almost to zero, and the smooth distribution of $B(x, t)$ in the growing resistive domain can be regarded as a giant multiquanta vortex-antivortex dipole.

In the more nonlocal regime of $\lambda / \lambda_{J}=\sqrt{10}$, the result of simulation in Fig. 43a shows $\theta(x-v t)$ in a vortex for different current densities at $\eta=0.1$. Similar to $\theta(x)$ shown in Fig. 39 of the previous case, a uniformly moving vortex in a nonlocal junction produces a trailing tail of Cherenkov electromagnetic waves, the amplitude and wavelength of these waves increase with the driving current. The vortex becomes unstable at a threshold current $\beta_{s}=0.61$ as the peak amplitude of Cherenkov waves reaches $\theta_{c}$ and the first vortex-antivortex pair appears. The subsequent evolution of expanding phase pile is similar to that is described in the first case: vortices accumulate at the left edge, antivortices accumulate at the right edge, and the remaining dissociated vortices and antivortices go through each other in the middle, as shown in Fig. 43b. After generation of multiple vortex-antivortex pairs, the speed of propagation of the phase pile edges increases as compared to the initial unstable vortex: from about $0.38 c_{s}$ to about $0.9 c_{s}$ for $\eta=0.1$ and $\lambda / \lambda_{J}=\sqrt{10}$. A 3D image of this process 
is shown in Fig. 44 as well. Notice that the phase difference $\theta(\infty)-\theta(-\infty)=2 \pi$ between the edges of the growing phase pile also remains fixed.

Magnetic field distribution $B(x)$ in the expanding phase pile shown in Fig. 45 has large Cherenkov radiation peaks at the leading edges but becomes rather smooth in the middle, similar to that of Fig. 42.

Shown in Fig. 46 are the steady-state vortex velocities $v(\beta)$ calculated for the bulk junction using the result of simulations of (86) in above cases as well as (44). The instability corresponds to the endpoints of the $v(\beta)$ curves which have two distinct parts. At small $\beta \lesssim \eta$ the velocity $v(\beta)$ increases sharply with a slope limited by a weak quasiparticle viscous drag. At larger $\beta \gtrsim \eta$ the increase of $v(\beta)$ with $\beta$ slows down, as the vortex velocities are mostly limited by radiation friction [96] and depend weakly on the form of dissipative terms in (75). For a low- $J_{c}$ junction with $\lambda_{J} / \lambda=10$, the effect of Cherenkov radiation on $v(\beta)$ is weak, but for a high- $J_{c}$ bulk junction with $\lambda / \lambda_{J}=\sqrt{10}$ and $\eta \ll 1$, radiation friction dominates at practically all $\beta$, significantly reducing both $v(\beta)$ and $\beta_{s}$. The calculated $v(\beta)$ curve shown in Fig. 46 illustrates the effect of radiation friction which limits the velocity of the vortex in the most part of the stability region $0<\beta<\beta_{s}$, except for small $\beta<\eta$ where $v$ is limited by the conventional ohmic viscous drag.

\subsubsection{THIN FILM EDGE JUNCTION}

For an edge Josephson junction in a thin film of thickness $W \ll \lambda$ (see Fig. 38(b)), the effects of electromagnetic nonlocality are greatly enhanced by the long-range stray magnetic fields screened over the large Pearl length $\lambda_{P}=2 \lambda^{2} / W \gg \lambda$. In the thin film limit $\lambda_{P} \gg \lambda$, the equation in (75) using the kernel $G(x)$ given by (78) becomes

$$
\ddot{\theta}+\eta \dot{\theta}=\frac{l_{J}}{2} \int_{-\infty}^{\infty}\left[\mathbf{H}_{\mathbf{0}}\left(\frac{|x-u|}{\lambda_{P}}\right)-Y_{0}\left(\frac{|x-u|}{\lambda_{P}}\right)\right] \frac{\partial^{2} \theta}{\partial u^{2}} d u-\sin \theta+\beta,
$$

where $\mathbf{H}_{0}(x)$ and $Y_{0}(x)$ are the zero order Struve and Bessel functions, as mentioned previously. The behavior of the vortex solution is controlled by the ratio of two characteristic lengths $l_{J}=\lambda_{J}^{2} / \lambda$ and $\lambda_{P}$. Yet, the limit of $\lambda / \lambda_{P} \rightarrow 0$ does not reduce to the sine-Gordon equation in (44) at any ratio of $l_{J} / \lambda_{P}$ because $G(x)$ decreases as $1 / x$ at $x>\lambda_{P}$, and the integral $\int_{0}^{\infty} G(x) d x$ with $G(x)$ defined by (78) diverges logarithmically. The crossover of (87) to the sine-Gordon local Josephson electrodynamics for very low- $J_{c}$ edge junctions requires taking into account the effects of finite film thickness [97] which we do not consider here.

Our simulation of (87) with $\eta=0.1$ and $l_{J} / \lambda_{P}=1 / 2$ revealed the dynamics of vortex instability and the subsequent generation of vortex-antivortex pairs at $\beta>\beta_{s}$ similar to that 

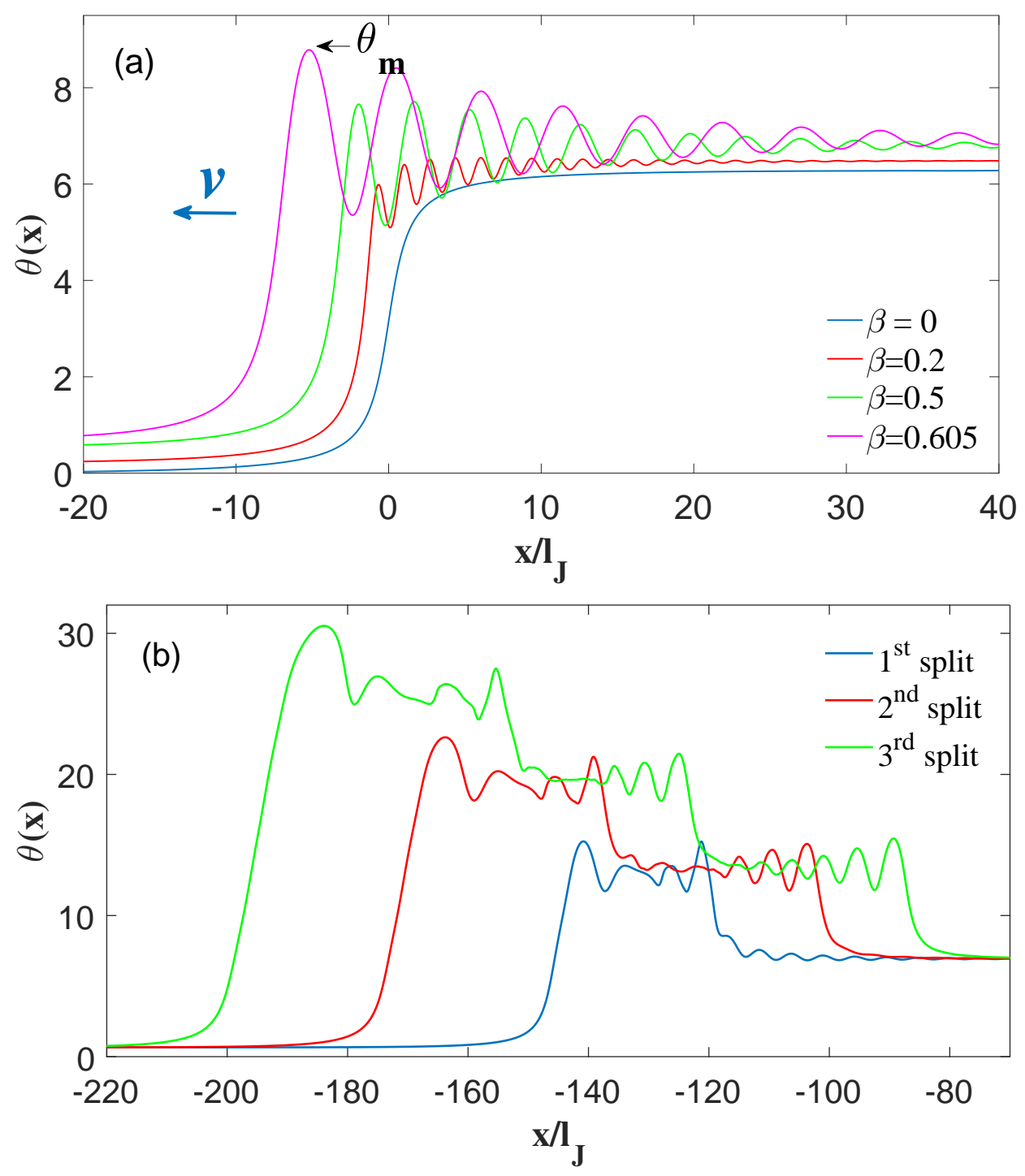

FIG. 43: Phase profiles before and after instability calculated for a bulk junction with $\eta=0.1$ and $\lambda / \lambda_{J}=\sqrt{10}$. (a) profiles of $\theta(x-v t)$ in a uniformly moving stable vortex at different values of $\beta$ up to $\beta=0.605$. (b) At $\beta_{s}=0.61$ the highest maximum of Cherenkov waves reaches a critical value of $\theta_{c}=8.79$ and starts growing and developing an expanding vortexantivortex pair. Depicted are the snapshots of the first three splittings of the unstable vortex into vortex-antivortex pairs at $\beta=\beta_{s}$. 


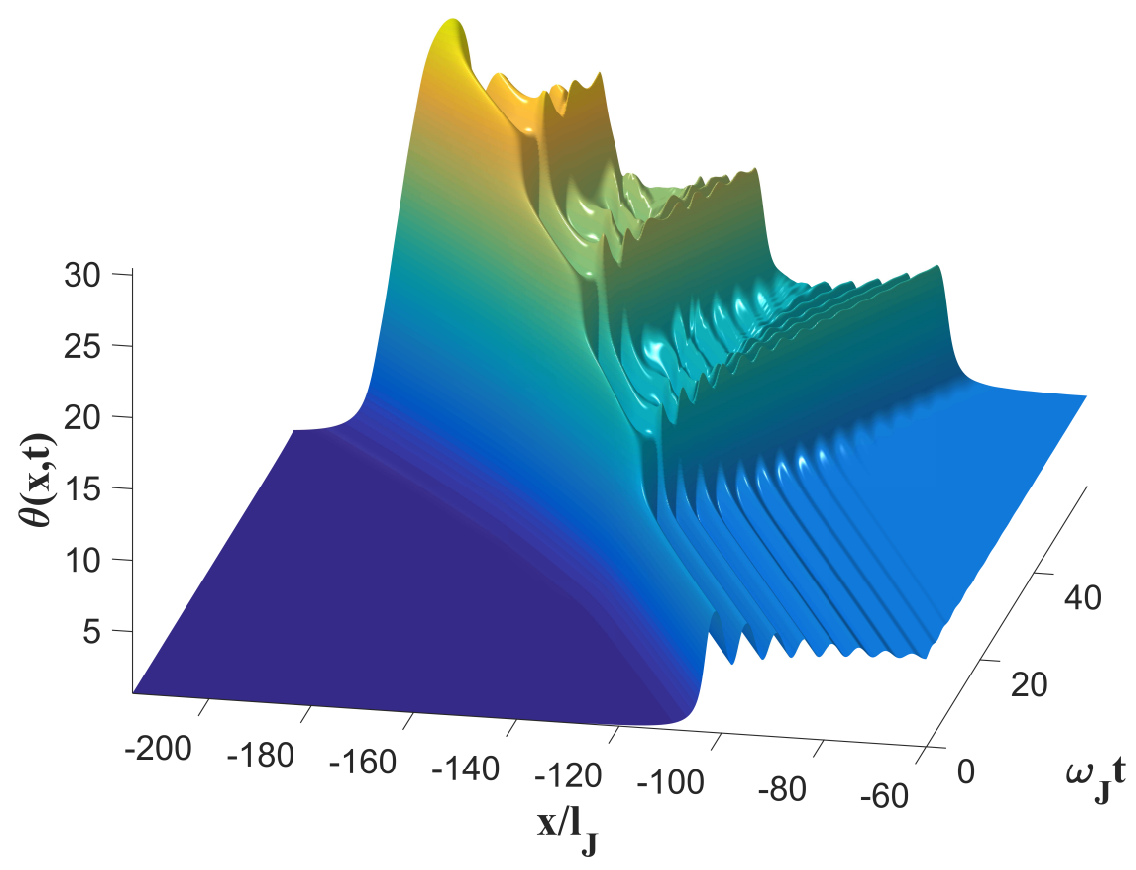

FIG. 44: A 3D image of vortex instability and the initial stage of the phase pile formation calculated at $\beta=\beta_{s}=0.61$ for a high- $J_{c}$ bulk junction with $\lambda / \lambda_{J}=\sqrt{10}$ and $\eta=0.1$. Here $\theta(x, t)$ in the vortex at $\beta=\beta_{s}$ was computed by solving the full dynamic equation in (86) with the initial distribution equal to the stable single-vortex solution $\theta(x-v t)$ calculated at $\beta=\beta_{s}-0.01$. As a result, the vortex then accelerates slightly and becomes unstable, triggering the growth of the phase pile. After multiple generations of vortex-antivortex pairs, vortices at the leading edges reach velocities of $90 \%$ of $c_{s}$.

in a bulk junction. For instance, Fig. 47 shows the dynamics of splitting of the Josephson vortex and its evolution into expanding phase pile at $\beta_{s}=0.63$. The velocity-current $v(\beta)$ curve for a vortex in this edge junction geometry is shown in Fig. 48.

The simulation movies of driven steady-state vortices with trailing tails of Cherenkov radiation, the initial stage of instability development, and the dynamics of phase pile formation are shown in Ref.[116]. For $l_{J}=\lambda_{P} / 2$ and $\eta=0.025$, the onset of instability corresponds to $\beta_{s}=0.475$ and $\theta_{c}=8.84$. In the extreme nonlocal limit of $l_{J} / \lambda_{P} \rightarrow 0$ corresponding to the logarithmic kernel (79) of section 4.2.1, we obtained $\beta_{s}=0.55$ and $\theta_{c}=8.83$ at $\eta=0.05$, and $\beta_{s}=0.905$ and $\theta_{c}=8.65$ at $\eta=0.5$. Interestingly, the dynamics of instability in an edge junction changes, depending on the damping parameter $\eta$. For larger $\eta=0.5$, the only significant trailing Cherenkov hump in $\theta(x-v t)$ becomes unstable and starts growing. However, it turns out that in the weak damping limit $(0.025<\eta<0.05)$, the second trailing peak in a weakly decaying Cherenkov wave has a higher amplitude than the first one. In 


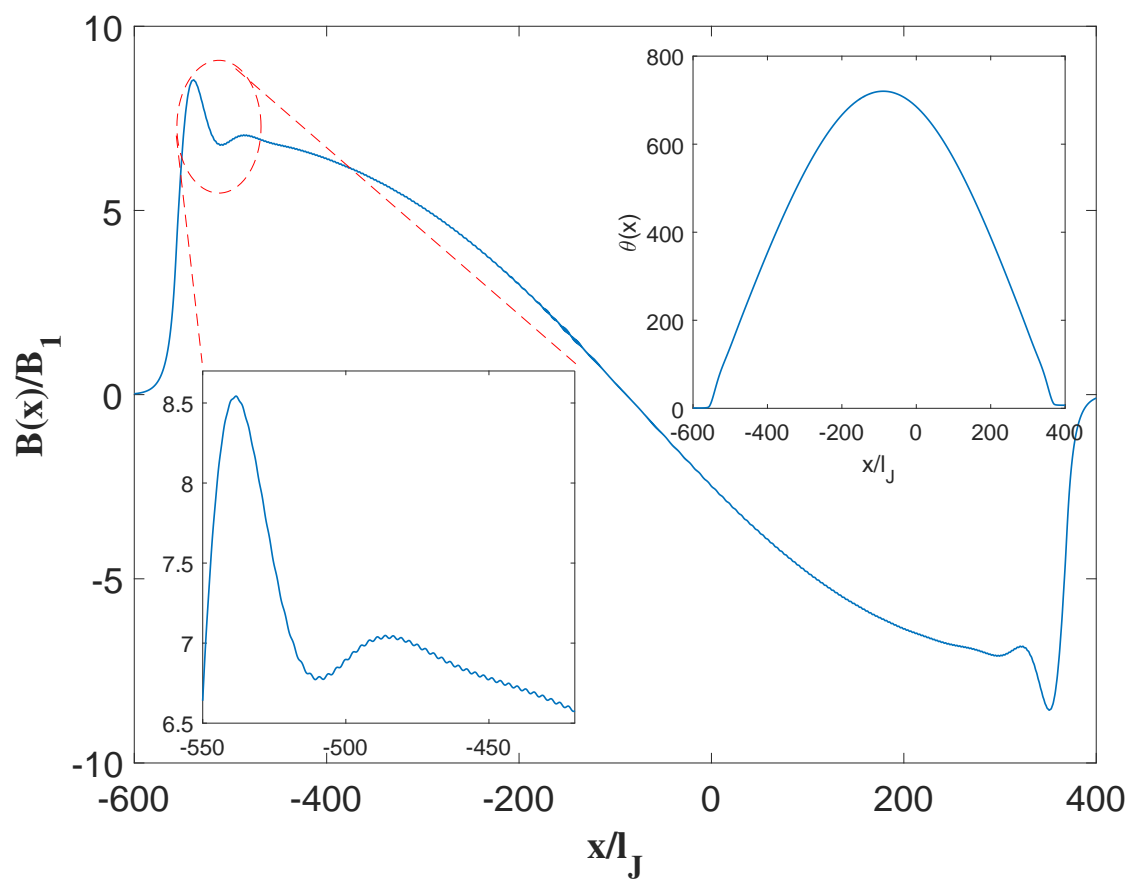

FIG. 45: A snapshot of the magnetic field $B(x)$ in a phase pile calculated for a bulk high- $J_{c}$ junction at $\beta=\beta_{s}=0.61, \eta=0.1, \lambda / \lambda_{J}=\sqrt{10}$, and $B_{1}=\Phi_{0} / 2 \pi \lambda \lambda_{J}$. The large peaks in $B(x)$ at the leading edges result from emerging vortex-antivortex pairs generating strong Cherenkov waves, the amplitude of which diminishes greatly in the bulk of the dissipative domain where vortices overlap strongly. Inset shows a snapshot of the corresponding phase profile. The growing phase pile with $\theta(x) \gg 1$ causes small ripples in $B(x)$ due to rapidly oscillating Josephson currents $\propto \sin \theta(x)$ clearly visible in the zoomed structure of the leading edge.

this case the instability is triggered by the second Cherenkov peak which starts growing and pushing up the neighboring peaks on both sides, causing them to merge and form an expanding vortex-antivortex pair, as shown in simulation videos in $\operatorname{Ref}[116]$. The results of the $v(\beta)$ curves calculated in the extreme nonlocal limit for different damping parameters is plotted in Fig. 49. The maximum velocity $v_{s}=v\left(J_{s}\right)$ at which the steady-state moving vortex remains stable, can be written in the scaling form $v_{s}=c_{s} \lambda_{J} f(\eta) / \lambda$, where $f(\eta)$ decreases from $\simeq 2.5$ at $\eta=0.5$ to $\simeq 1$ at $\eta=10^{-3}$. The Josephson vortex in thin film edge junctions can reach the velocities exceeding the nominal Swihart velocity $c_{s}=\omega_{J} \lambda_{J}$ at $J \simeq J_{s}$ if $\lambda_{J}>\lambda$ but $l_{J}<\lambda_{P}$, that is, $W<2 \lambda^{3} / \lambda_{J}^{2}$. Dynamics of $\theta(x, t)$ in the nonlocal limit at $J>J_{s}$ is similar to that is shown in Fig. 44, except that the edges of phase pile can propagate with "superluminal" velocities $v \simeq v_{s}>c_{s}$ if $\lambda_{J}>\lambda$. Once vortex-antivortex pairs start replicating, the speed of leading vortices at the edges gradually increases from 


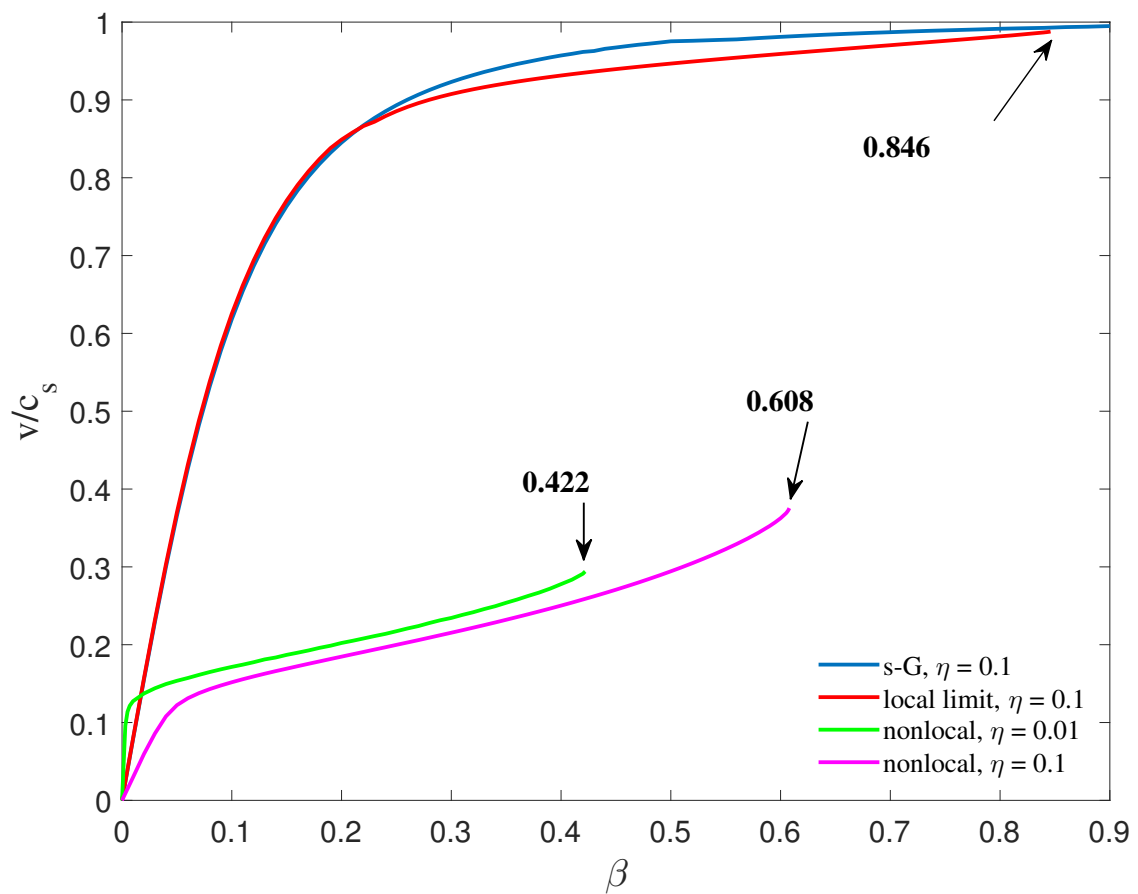

FIG. 46: Velocities of a stable singe vortex $v(\beta)$ as functions of current calculated for different junction geometries. The instability occurs at the endpoints (shown by arrows) of the $v(\beta)$ curves. The graph shows $v(\beta)$ for a bulk junction calculated from (86) at $\eta=0.1$ in the seemingly conventional weak-link limit, $\lambda_{J} / \lambda=10$ (for comparison, the blue curve shows $v(\beta)$ calculated from $(44)$ at $\eta=0.1)$. The magenta and green curves show $v(\beta)$ calculated for a bulk nonlocal junction with $\lambda / \lambda_{J}=\sqrt{10}$ for values of $\eta=0.1$ and $\eta=0.01$, respectively. 


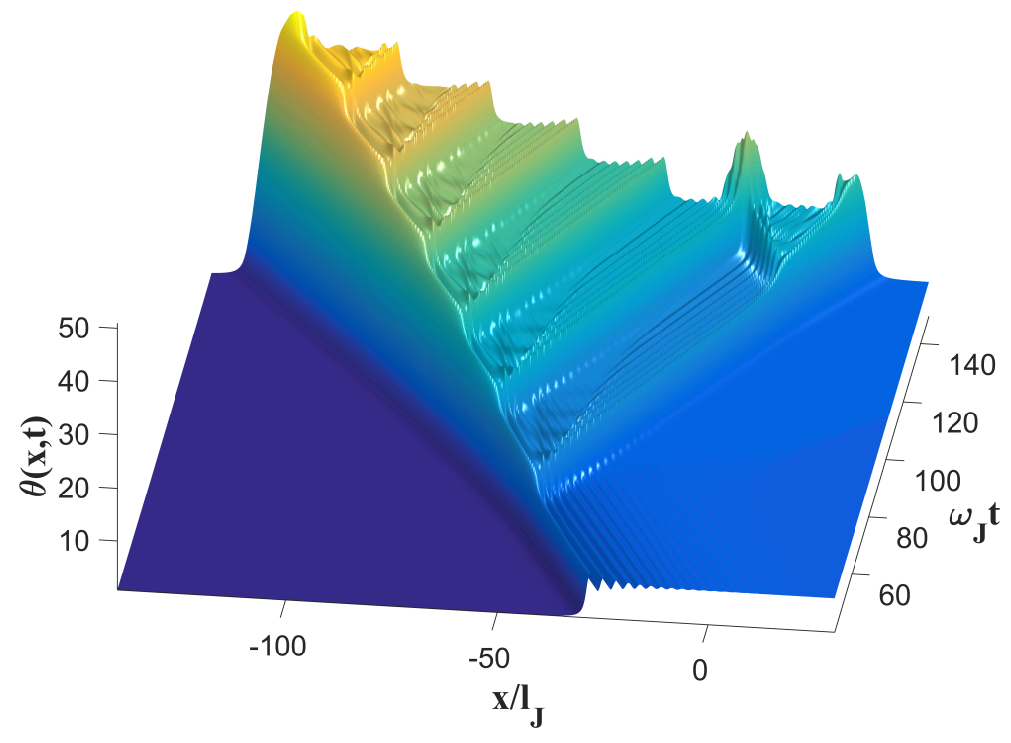

FIG. 47: A 3D image of vortex instability and the initial stage of the phase pile formation calculated at $\beta=\beta_{s}=0.63$ for a thin film edge junction with $\eta=0.1$ and $l_{J}=\lambda_{P} / 2$. Here $\theta(x, t)$ in the vortex at $\beta=\beta_{s}$ was computed by solving the full dynamic equation in (87) with the initial distribution equal to the stable single-vortex solution $\theta(x-v t)$ calculated at $\beta=\beta_{s}-0.01$. As a result, the vortex then accelerates slightly and becomes unstable, triggering the growth of the phase pile. After multiple generations of vortex-antivortex pairs, vortices at the leading edges reach velocities of $1.12 l_{J} \omega_{J}$.

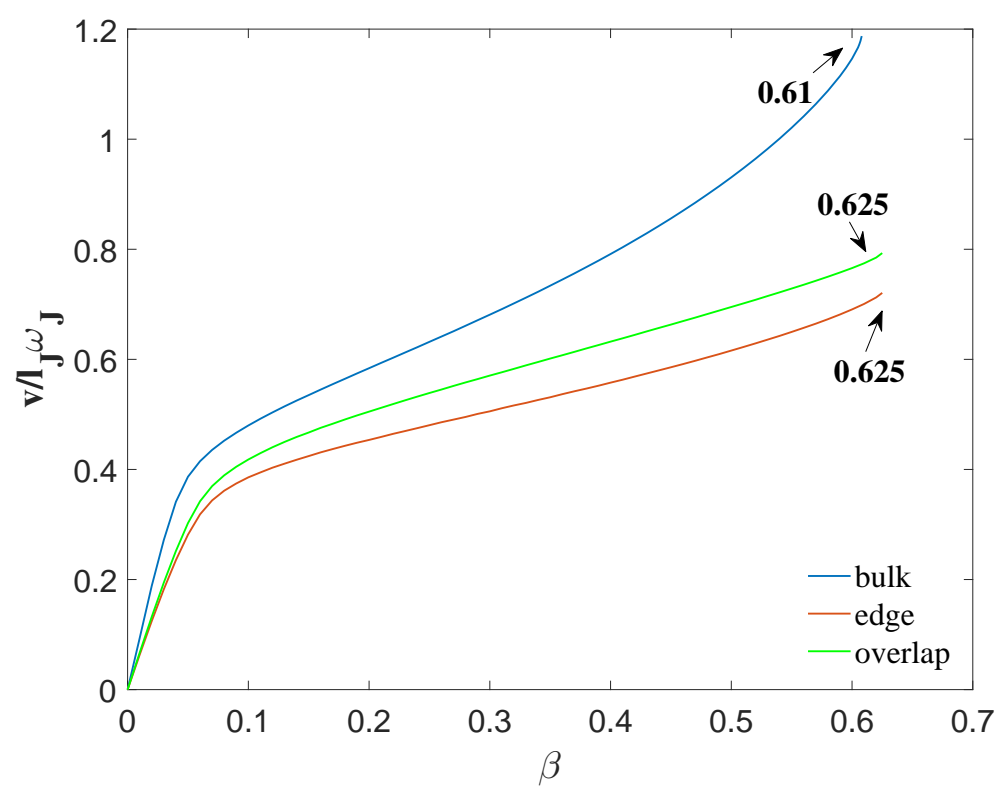

FIG. 48: Vortex velocity $v(\beta)$ as a function of driving current $\beta=J / J_{c}$ calculated at $\eta=0.1$ for different geometries: nonlocal bulk junction with $\lambda / \lambda_{J}=\sqrt{10}$ (blue), thin film edge junction with $l_{J}=\lambda_{P} / 2$ (red) and thin film overlap junction with $l_{J}=W$ (green). For small currents $(\beta<0.05)$, the velocity $v(\beta)$ in all cases is mostly limited by the ohmic viscose drag while the Cherenkov radiation friction force takes over at larger $\beta$. 


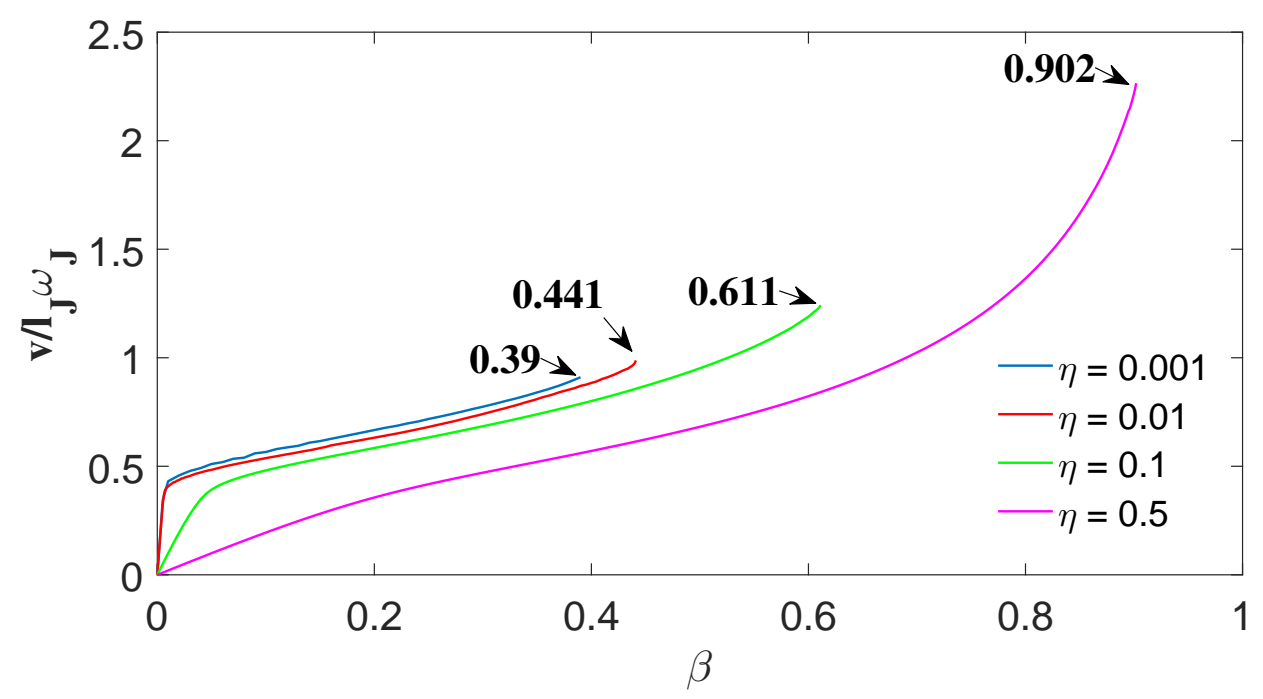

FIG. 49: The graph shows results for a thin film edge junction in the extreme nonlocal limit described by (80). Notice that both the $v(\beta)$ curves and the critical values $\beta_{s}$ at $\eta=0.1$ and $\eta=0.01$ for the thin film junction are close to those for the nonlocal bulk junction shown in Fig. 46. This is because for a nonlocal bulk junction, $\theta^{\prime \prime}(u)$ in (75) has a sharp peak of width $\sim\left(\lambda_{J} / \lambda\right)^{2} \lambda=0.1 \lambda$ so $G\left[\lambda_{J}(x-u) / \lambda\right]=\pi^{-1} K_{0}\left[\lambda_{J}|x-u| / \lambda\right]$ can be approximated by its expansion at small argument, $K_{0}\left(\lambda_{J} x / \lambda\right) \rightarrow \ln \left(2 \lambda / \lambda_{J}|x|\right)-0.577$, which reduces to (79). Here any constant factor under the log does not affect $\theta(x, t)$ since $\theta^{\prime}(-\infty)=\theta^{\prime}(\infty)=0$.

$v_{s}$ to a limiting value $v_{\infty}$, for instance, from $v_{s} \approx 0.72 l_{J} \omega_{J}$ to $v_{\infty} \approx 1.12 l_{J} \omega_{J}$ for an edge junction with $l_{J}=\lambda_{P} / 2$ and $\eta=0.1$. The graph clearly shows a gradual transition in the behavior of curves toward the exactly solvable case of (80) in overdamped regime for which the I-V characteristics have been previously calculated by Gurevich[94].

\subsubsection{THIN FILM OVERLAP JUNCTION}

We also considered overlap junctions in films of thickness $W<\lambda$ shown in Fig. 38(c). In this case the kernel $G(x)$ in (75) is given by [102]

$$
G(x)=\pi^{-1} \ln \operatorname{coth}\left(\frac{\pi}{4 W}|x|\right)
$$

and the full dynamic equation becomes,

$$
\ddot{\theta}+\eta \dot{\theta}=\frac{l_{J}}{\pi} \int_{-\infty}^{\infty} \ln \operatorname{coth}\left(\frac{\pi}{4 W}|x-u|\right) \frac{\partial^{2} \theta}{\partial u^{2}} d u-\sin \theta+\beta .
$$

The kernel $G(x)$ defined by (88) behaves qualitatively similar to the bulk kernel in (86), but decreases exponentially over the length $2 W / \pi$ smaller than $\lambda$. As a result, the nonlocal 


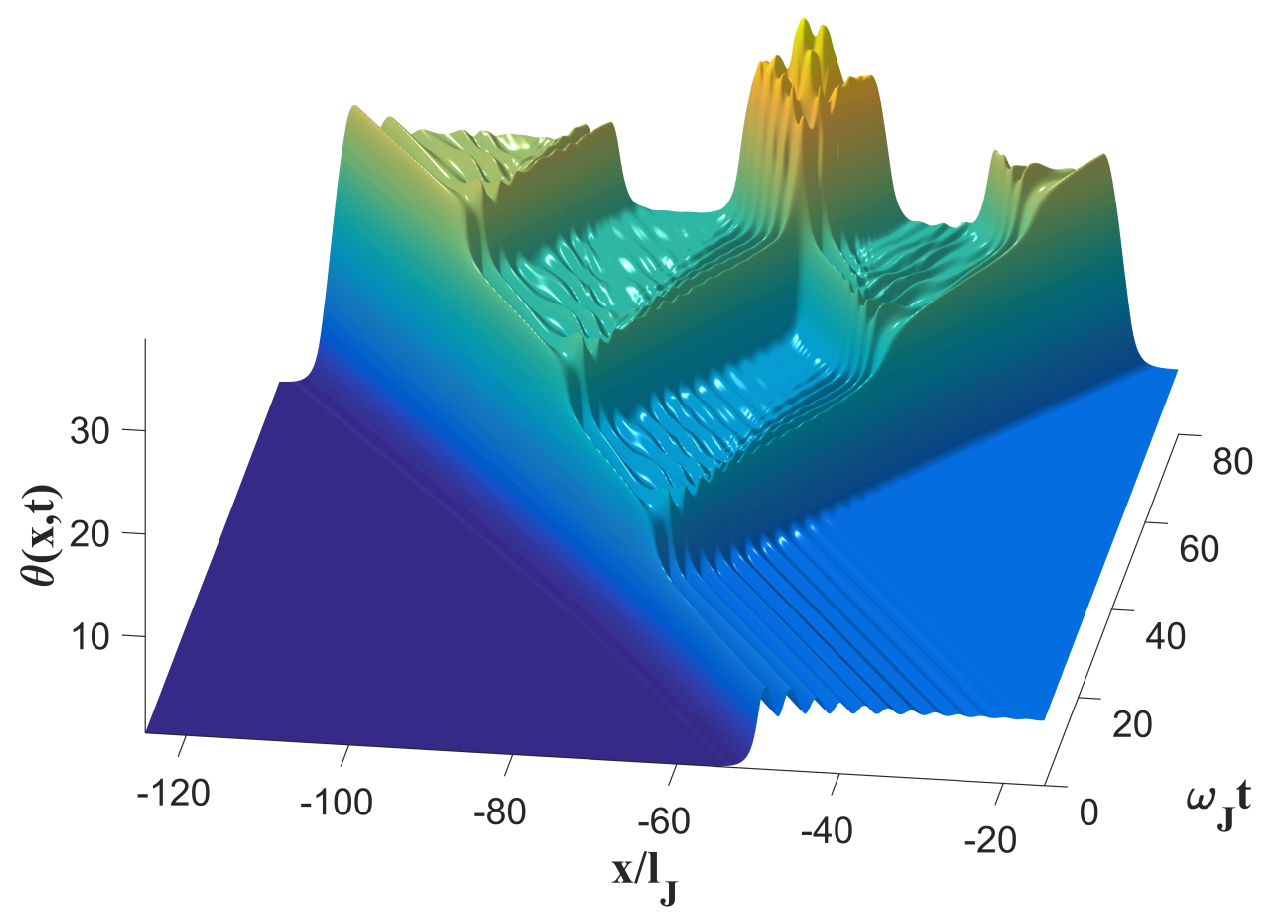

FIG. 50: A 3D image of vortex instability and the initial stage of formation of phase pile at $\beta=\beta_{s}=0.63$ in an overlap junction at $\eta=0.1$ and $l_{J}=W$. Here $\theta(x, t)$ in the vortex at $\beta=\beta_{s}$ was computed by solving the full dynamic equation in (89) with the initial distribution equal to the stable single-vortex solution $\theta(x-v t)$ calculated at $\beta=\beta_{s}-0.01$. As a result, the vortex then accelerates slightly and becomes unstable, triggering the growth of the phase pile. After generation of many vortex-antivortex pairs, the leading edges of the phase pile reach the velocity close to $0.94 l_{J} \omega_{J}$.

effects in the overlap junction are diminished as compared to a bulk junction and are much suppressed as compared to a thin film edge junction. Yet even in this seemingly conventional case described by the sine-Gordon equation with $\lambda_{J} \simeq\left(\Phi_{0} / 8 \mu_{0} W J_{c}\right)^{1 / 2}$, the nonlocal effects in overlap junctions can manifest themselves at large vortex velocities, producing Cherenkov radiation and the vortex splitting instability similar to the cases discussed above. For instance, Fig. 50 shows the instability of the single vortex and the initial stage of the phase pile evolution calculated for an overlap junction with $\eta=0.1$ and $l_{J}=W$. Shown in Fig. 48 is the calculated $v(\beta)$ curve which illustrates the effect of the Cherenkov radiation force on the velocity of the vortex.

The simulation movies of driven steady-state vortices with trailing tails of Cherenkov radiation and the dynamics of phase pile formation are shown in associated simulation videos in Ref.[116]. 


\subsection{INSTABILITY ANALYSIS}

Now we turn to the instability of a traveling vortex solution $\theta_{0}(x-v t)$ disturbed by a small perturbation $\delta \theta(x, t)=g(x-v t) e^{\gamma t}$. Substituting $\theta=\theta_{0}+\delta \theta$ into $(75)$ and linearizing it with respect to $\delta \theta \ll 1$, we obtain the following eigenvalue equation for the increment $\gamma$,

$$
\left(\gamma^{2}+\eta \gamma\right) g-2 v \gamma g^{\prime}=\hat{\Pi}\{g\}-g \cos \theta_{0}(\zeta), \quad g( \pm \infty)=0
$$

where the prime denotes differentiation with respect to $\zeta=x-v t$, and $\hat{\Pi}$ is the following integro-differential operator

$$
\hat{\Pi}\{g\}=l_{J} \int_{-\infty}^{\infty} G\left(\frac{\left|\zeta-\zeta_{1}\right|}{\Lambda}\right) g^{\prime \prime}\left(\zeta_{1}\right) d \zeta_{1}+\eta v g^{\prime}(\zeta)-v^{2} g^{\prime \prime}(\zeta) .
$$

The vortex solution $\theta_{0}(\zeta)$ is unstable if there is at least one eigenvalue with $\operatorname{Re}(\gamma)>0$, so the disturbance $\delta \theta=g(\zeta) e^{\gamma t}$ grows exponentially.

Given the complexity of the traveling vortex solutions $\theta_{0}(\zeta)$ shown in Fig. 39, the eigenvalue problem defined by (90)-(91) can only be solved numerically. Our simulations of the nonlinear integral equation in (75) have shown that the instability originates at the highest maximum $\theta=\theta_{m}$ of the trailing Cherenkov wave as $\theta_{m}$ exceeds a critical value $\theta_{c}$ which ranges between 8.65 and 8.84 , depending on the values of $\eta$ and $\lambda_{J} / \lambda$, as shown above for different junction geometries. Here $\theta_{c}$ lies in the region of $5 \pi / 2<\theta<3 \pi$ where a uniform state of the Josephson junction $(k \rightarrow 0)$ is unstable and $\cos \theta_{\infty}<0$ in (83). At the instability threshold $\beta=\beta_{s}$, the first highest peak of amplitude $\theta_{m}(t)$ starts growing and merging with the neighboring peak in $\theta(x, t)$, forming an expanding vortex-antivortex pair, as shown in the detailed simulation movies presented in Ref.[116]. Equations (84)-(85) show that the nonlocality of (75) results in Cherenkov radiation behind a uniformly moving Josephson vortex even in a weakly-coupled junction $\lambda \ll \lambda_{J}$ which is usually described by the sine-Gordon equation in (44). Thus, the approximation of (75) with (44) can miss essential effects in the dynamics of Josephson vortices.

\subsection{GENERATION OF QUASIPARTICLES BY A MOVING VORTEX}

The effects reported here are most pronounced in underdamped SIS junctions between s-wave superconductors at low temperatures for which the viscous drag coefficient $\eta \propto \exp (-\Delta / T)$ due to thermally-activated quasiparticles [42] is small. Weak dissipative drag $\eta<1$ implies that the maximum Josephson voltage $V=\hbar v / e \mathcal{L}$ produced by a vortex of length $\mathcal{L}$ moving with the velocity $v$ does not generate quasi-particles, that 
is $e V<\Delta$. Consider first a bulk junction with $\lambda<\lambda_{J}$ where $\mathcal{L}=\lambda_{J} \sqrt{1-v^{2} / c_{s}^{2}}$ and $\lambda_{J}=\left(\Phi_{0} / 4 \pi \mu_{0} \lambda J_{c}\right)^{1 / 2} \simeq \xi\left(\kappa J_{d} / J_{c}\right)^{1 / 2}$ [42]. Here $\kappa=\lambda / \xi$ is the GL parameter, $J_{d}=\Phi_{0} / 3^{3 / 2} \pi \mu_{0} \lambda^{2} \xi$ is the depairing current density, and $c_{s}=c\left(d_{j} / 2 \epsilon_{r} \lambda\right)^{1 / 2}$ is the Swihart velocity in a Josephson contact with the thickness $d_{j}$ and the dielectric constant $\epsilon_{0} \epsilon_{r}$. The condition of $e V<\Delta$ can be written in the form

$$
\frac{\xi_{0}}{\xi}\left(\frac{d_{j} J_{c}}{\kappa \epsilon_{r} \lambda J_{d}}\right)^{1 / 2} \leq \frac{v_{F}}{c}\left(1-\frac{v^{2}}{c_{s}^{2}}\right)^{1 / 2} .
$$

Here the superconducting gap $\Delta$ was expressed in terms of the clean-limit coherence length, $\xi_{0}=\hbar v_{F} / \pi \Delta$, where $v_{F}$ is the Fermi velocity. As an example, we take a clean $\mathrm{Nb}_{3} \mathrm{Sn}$ with $\xi=\xi_{0}$ and $\kappa=20$ for which $\lambda_{J}=10 \lambda$ used in our simulations corresponds to $J_{d} / J_{c} \simeq$ $10^{2} \kappa=2 \cdot 10^{3}$. For these numbers and $\epsilon_{r}=10$ and $d_{j} / \lambda=10^{-2}$, the left-hand side of (92) is $\simeq 10^{-4}$. For the ratio $v_{F} / c \simeq 10^{-3}[117]$ and the velocity of splitting $v / c_{s} \approx 0.98$ taken from Fig. 43 , the right-hand side of (92) is about $2 \cdot 10^{-4}$, that is, the condition (92) is satisfied.

Now we turn to the nonlocal limit $l_{J}<\Lambda$ and evaluate the maximum voltage in the AJ vortex $V=\hbar v \theta_{m}^{\prime} / 2 e l_{J}$, where $l_{J}=\lambda_{J}^{2} / \lambda=\left(3^{3 / 2} J_{d} / 4 J_{c}\right) \xi$, and $\theta_{m}^{\prime}$ is the maximum value of the derivative $\theta^{\prime}\left[(x-v t) / l_{J}\right]$ in the moving vortex. The condition, $\mathrm{eV}<\Delta$ then takes the form

$$
\frac{v}{v_{F}}<\frac{3^{3 / 2} \xi J_{d}}{2 \pi \theta_{m}^{\prime} \xi_{0} J_{c}}
$$

As follows from the numerical results shown in Fig. 39, the value of $\theta_{m}^{\prime} \approx 2.5$ at the critical splitting velocity $v=v_{m} \simeq l_{J} \omega_{J}$ is not much larger than $\theta_{m}^{\prime}=2$ for a static vortex. Treating the edge Josephson junction of width $d_{j} \ll L$ in the middle of a thin film of width $L$ and thickness $W<d_{j}$ as a coplanar strip line on an infinite dielectric substrate, we define formally $c_{s}=\left(2 e J_{c} / \hbar C\right)^{1 / 2} \lambda_{J}$, where the capacitance per unit edge area is $C \simeq \epsilon_{0}\left(1+\epsilon_{r}\right) \ln \left(4 L / d_{j}\right) / \pi W[118,119]$. Hence,

$$
v_{m} \simeq c\left[\frac{W J_{d}}{\kappa \lambda J_{c}\left(1+\epsilon_{r}\right) \ln \left(4 L / d_{j}\right)}\right]^{1 / 2} .
$$

Let us evaluate $v_{m}$ for a monolayer edge junction $[113,114]$ on a $\mathrm{Si}$ substrate with the dielectric constant $\epsilon_{r}=10$. For $W / \kappa \lambda=10^{-3}, d_{j}=10^{-3} L$, and $J_{c}=10^{-2} J_{d}$, (94) yields, $v_{m} \sim 3 \cdot 10^{-2} c$. Combining (93) and (94) gives the condition that a moving AJ vortex does not generate quasiparticles at all velocities $v<v_{m}$,

$$
\frac{J_{c}}{J_{d}}<0.03\left(\frac{v_{F}}{c}\right)^{2} \frac{\lambda^{2} \xi}{\xi_{0}^{2} W}\left(1+\epsilon_{r}\right) \ln \frac{4 L}{d_{j}} .
$$

Taking here $v_{F}=1.82 \cdot 10^{6} \mathrm{~m} / \mathrm{s}$ for $\mathrm{Pb}[120], W \xi_{0}^{2} / \xi \lambda^{2}=10^{-3}, d_{j}=10^{-3} L$, and $\epsilon_{r}=10$, we obtain that (95) is satisfied if $J_{c}<0.1 J_{d}$. Thus, the strong electromagnetic nonlocality of 
thin film edge junctions extends the region of the parameters where a moving vortex does not generate quasiparticles, which increases $\eta$ and suppresses Cherenkov radiation. Moreover, the condition of $e V<\Delta$ can be extended to even higher $J_{c}$ if an edge thin film Josephson junction on a dielectric substrate of thickness $h$ is placed on a grounded metallic plate. In this case the effective capacitance of the stripline can be significantly increased [119], which reduces $c_{s}$ so that the condition of $e V<\Delta$ can be satisfied even at $J_{c} \simeq J_{d}$.

The conditions (92)-(95) imply no significant suppression of $\Delta(x)$ in the AJ vortex core. To see how well this requirement is satisfied, we evaluate the variation of $\Delta(x)$ using the GL theory in which $\Delta^{2}(x)=\left[1-\xi^{2} Q^{2}(x)\right] \Delta_{0}^{2}$, where $\Delta_{0}$ is the equilibrium gap, and $\mathbf{Q}=$ $\nabla \varphi+2 \pi \mathbf{A} / \Phi_{0}$ is the gauge-invariant phase gradient produced by circulating vortex currents outside the SIS junction. In the first approximation, we take here $Q_{x}=l_{J} \operatorname{sign}(y) /\left(x^{2}+l_{J}^{2}\right)$ and $Q_{y}=-x /\left(x^{2}+l_{J}^{2}\right)$ in the AJ vortex at $x \ll \Lambda[76]$ and obtain

$$
\Delta(x) \simeq\left[1-\frac{\xi^{2}}{2\left(x^{2}+l_{J}^{2}\right)}\right] \Delta_{0} .
$$

The maximum gap reduction, $\left[\Delta_{0}-\Delta(0)\right] / \Delta_{0}=\xi^{2} / 2 l_{J}^{2}=8 J_{c}^{2} / 27 J_{d}^{2}$, at $x=0$ drops below $1 \%$ if $J_{c}<0.2 J_{d}$.

\subsection{POWER GENERATED BY A MOVING JOSEPHSON VORTEX}

We evaluate the power $P=W \int_{-\infty}^{\infty} V^{2}(\zeta) d \zeta / R$ dissipated by the moving vortex in a thin film junction, where $V=\hbar \dot{\theta} / 2 e=-v \hbar \theta^{\prime}(\zeta) / 2 e$ is the Josephson voltage, and $\zeta=x-v t$. In the nonlocal limit at $\eta<1$ our numerical simulations have shown that $\theta(\zeta)$ can be satisfactory described by $\theta(\zeta)=\pi+2 \tan ^{-1}\left(\zeta / l_{J}\right)$ for the AJ vortex [76]. Then

$$
P=\frac{\hbar^{2} v^{2} W}{4 e^{2} R} \int_{-\infty}^{\infty} \theta^{\prime 2}(\zeta) d \zeta \simeq \frac{v^{2} \Phi_{0}^{2} W}{2 \pi l_{J} R}
$$

where $l_{J}=\lambda_{J}^{2} / \lambda$. At the maximum velocity $v_{s} \simeq l_{J} \omega_{J}$ at which the uniformly moving vortex becomes unstable, the power $P_{s}=P\left(v_{s}\right)$ becomes

$$
P_{s} \simeq \frac{\Phi_{0}^{2} W}{4 \pi \mu_{0} \lambda^{2} R C},
$$

where we used that $\left(\omega_{J} \lambda_{J}\right)^{2}=1 / 2 \mu_{0} \lambda C$. Notice that $P_{s}$ is independent of $J_{c}$. For estimates of $P_{s}$, it is convenient to express $R$ in terms of the dimensionless dissipation parameter $\eta=1 / R C \omega_{J}$ used in our numerical simulations of $\theta(x, t)$. Then (98) takes the form

$$
P_{s} \simeq \eta \varepsilon_{0} W \omega_{J}
$$


Here $\varepsilon_{0}=\Phi_{0}^{2} / 4 \pi \mu_{0} \lambda^{2}$ is the characteristic energy of the Abrikosov vortex per unit length. Given that $C \simeq \epsilon_{0}\left(1+\epsilon_{r}\right) \ln \left(4 L / d_{j}\right) / \pi W[118,119]$, as was mentioned above, (99) suggests that the power $P_{s}$ decreases quadratically as the film thickness $W$ decreases, and becomes negligible in edge thin film SIS junctions at low temperatures as the quasiparticle resistance $R(T)$ grows exponentially as $T$ decreases. For an edge junction in a $\mathrm{Nb}$ film with $W=1 \mathrm{~nm}$, $\lambda=40 \mathrm{~nm}, \varepsilon_{0} \sim 10^{4} \mathrm{~K} / \mathrm{nm}$, and $\omega_{J}=100 \mathrm{GHz}$ much smaller than $\Delta / \hbar \simeq 2.4 \mathrm{THz}[8]$, (98) yields $P \sim 0.16 \mathrm{nW}$ at $\eta=10^{-2}$. Local overheating $\delta T=P Y_{K}$ caused by vortex dissipation is further reduced in thin film junctions for which the energy transfer to the substrate due to ballistic phonons is much more effective than diffusive phonon heat transport in thick samples, where $Y_{K}$ is the Kapitza interface thermal resistance [121]. Such weak overheating caused by a moving vortex cannot result in thermal bistability and hysteric switching due to hotspot formation [121].

\subsection{SUMMARY AND DISCUSSION}

We observed the phase pile dynamic state for different junction geometries and $\eta$ ranging from $10^{-3}$ to 0.5 . Proliferation of vortex-antivortex pairs triggered by a moving Josephson vortex can be essential for the physics and applications of superconducting structures like grain boundaries and Josephson junctions where the formation of expanding phase pile patterns can switch the entire junction into a normal state at currents well below the Josephson critical current, $J>J_{s} \simeq(0.4-0.7) J_{c}$. Such dynamic vortex instability can result in hysteretic jumps on the V-I curves which appear similar to those produced by heating effects $[43,122]$, yet this instability is affected by neither cooling conditions nor the nonequilibrium kinetics of quasiparticles. Indeed, heating is most pronounced in overdamped junctions with $\eta>1$ in which Cherenkov radiation is suppressed. By contrast, the Cherenkov instability is characteristic of the weakly-dissipative underdamped limit $\eta \ll 1$, although Fig. 49 shows that this instability in thin film edge junctions can persist up to $\eta=0.5$. Therefore, the crucial initial stage of the phase pile formation at $\eta \ll 1$ is unaffected by heating which may become more essential at the final stages of the transition of the entire junction into the normal state. At $\eta \sim 1$ the Cherenkov instability may be masked by heating effects, particularly in bulk junctions for which heat transfer to the coolant is less efficient than in thin films.

It should be emphasized that the instability reported here does not require special junctions with $J_{c} \sim J_{d}$. In fact, even for the seemingly conventional bulk junction with $\lambda_{J}=10 \lambda$ shown in Fig. 46, the instability at $J_{s} \simeq 0.846 J_{c}$ implies $J_{c} \sim 0.01 J_{d} / \kappa$, which translates 
into $J_{c} \sim 10^{-4} J_{d}$ for bulk $\mathrm{NbN}$ junctions. Moreover, in wide thin film edge junctions the nonlocality becomes important at even much lower $J_{c}$, as is evident from Fig. 49. Therefore, the effects reported here can occur in conventional underdamped junctions with $J_{c} \ll J_{d}$, particularly wide thin film or monolayer edge junctions. Interaction of Josephson vortices with pinned Abrikosov vortices in electrodes can result in additional mechanisms of splitting instability of Josephson vortices. For instance, radiation by Josephson vortices can be greatly enhanced as they move in a periodic magnetic potential of Abrikosov vortices along weak link grain boundaries [105, 123], whereas Abrikosov vortices trapped perpendicular to the Josephson junction can result in generation of Josephson vortex-antivortex pairs in the presence of the applied electric current [124].

Quantized vortex lines are quintessential topological defects [125, 126] which determine the behavior of superconductors and superfluids. Vortices in superconductors are characterized by an integer winding number $n$ in the phase $\varphi$ of the complex order parameter $\psi=\Delta \exp (i \varphi)$, singularity of $\nabla \varphi$ in a vortex core, and the quantized magnetic flux, $\Phi=n \Phi_{0}$. Because destruction of a topological defect requires overcoming a huge energy barrier proportional to the length or the area of a system, vortices can only disappear by annihilating with antivortices with the opposite sign of $n$ or exiting through the sample surface, or forming shrinking loops. This brings about the question: does this fundamental, topologically-protected stability of vortices remain preserved for a vortex driven by a strong force and, more generally, what happens to a global long-range order if a moving topological defect becomes unstable? To address this issue, we showed that a rapidly moving vortex can become unstable, causing a cascade of expanding vortex-antivortex pairs, which eventually destroy the global long-range order. This effect may impose limitations on the performance of Josephson memory [127, 128, 129], superconducting sources of THz radiation [122], or polycrystalline superconducting resonator cavities for particle accelerators [8], and have broader implications for other systems with long-range order.

Our results can be essential for other topological defects such as crystal dislocations or magnetic domain walls described by the generic nonlocal equation in (75) in which the integral term results from a common procedure of reduction of coupled evolution equations for several relevant fields to a single equation. For Josephson junctions, such coupled fields are $\theta$ and $B$, but for domain walls in ferromagnets, the nonlocality can result from longrange magnetic dipolar interactions [130]. For dislocations, the nonlocality and Cherenkov radiation of sound waves in (75) come from the discreteness of the crystal lattice [58] and long-range strain fields [57], although the dynamic terms in the Peierls equation [131, 132] are 
more complex than those in (75). Dynamic instabilities of dislocations have been observed in the lattice Frenkel-Kontorova models [58] in which sonic radiation can also result from periodic acceleration and deceleration of a dislocation moving in a crystal Peierls-Nabarro potential [57]. The latter effect becomes more pronounced as the dislocation core shrinks at higher velocities and becomes pinned more effectively by the lattice. By contrast, the instability reported here results entirely from Cherenkov radiation, the condition (83) can be satisfied for any system in which $G(k)$ in (83) decreases with $k$. This instability can thus have broader implications: for instance, the phase pile dynamics of Josephson vortices appears similar to a microcrack propagation caused by a continuous pileup of subsonic dislocations with antiparallel Burger vectors at the opposite tips of a growing crack described by (75) and (79) [57].

Our results give a new insight into breakdown of a global long-range order which has been usually associated with either thermally-activated proliferation of topological defects (like in the Berezinskii-Kosterletz-Thouless transition) or static arrays of quenched topological defects pinned by the materials disorder [126]. Here we point out a different mechanism in which a long-range order is destroyed as a single topological defect driven by a strong external force becomes unstable and triggers a cascade of expanding pairs of topological defects of opposite polarity. 


\section{CHAPTER 5}

\section{VORTEX DYNAMICS IN FINITE LENGTH}

\section{POLYCRYSTALLINE THIN FILM GRAIN BOUNDARIES}

Our previous results obtained for an infinitely long junction bring about the following issues related to the dynamics of vortices in junctions of finite length which are most relevant to experiments: 1 . What happens to AJ vortices driven by strong currents in a finite junction where in addition to the Cherenkov radiation, a vortex also radiates as it accelerates and decelerates due to its attraction to the edges of the junction? 2. How can the finite length of the junction affect the spontaneous generation of $\mathrm{v}$-av pairs produced by the radiation field of moving vortices? 3. How can the finite size effects change the structure of a static or moving AJ vortices, and whether they could cause a transition from a vortex to a phase slip state in which $\theta(t)$ becomes uniform along the junction? 4. What are the effects of Josephson nonlocality on the behavior of vortices driven by ac currents in finite junctions, as compared to the dynamics of Josephson vortices described by the sine-Gordon equation $[38,39,79,133]$ ? In this chapter, we are going to address these issues with the goal of presenting results of analytical and numerical investigations of the effect of finite junction length on the nonlinear dynamic of AJ vortices in the case of strong Josephson nonlocality. In particular, we investigate the electromagnetic response of a finite junction in a thin film in both perpendicular and parallel geometries under the dc and ac currents.

\subsection{ELECTRODYNAMICS OF FINITE JUNCTION IN NONLOCAL LIMIT}

Consider a junction of length $d$ in a film where a vortex is either perpendicular or parallel to the broad surface of the film, as shown in Fig. 51. Here Fig. 51 a is relevant to a polycrystalline superconducting screen in which the Josephson junction models a grain boundary perpendicular to the film, whereas Fig. $51 \mathrm{~b}$ represents an edge junction. To derive the equation for $\theta(x, t)$, we start with the superconducting current density:

$$
\begin{aligned}
& J_{x}=\frac{1}{\mu_{0} \lambda^{2}}\left(\frac{\Phi_{0}}{2 \pi} \frac{\partial \varphi}{\partial x}-A_{x}\right), \\
& J_{y}=\frac{1}{\mu_{0} \lambda^{2}}\left(\frac{\Phi_{0}}{2 \pi} \frac{\partial \varphi}{\partial y}-A_{y}\right),
\end{aligned}
$$



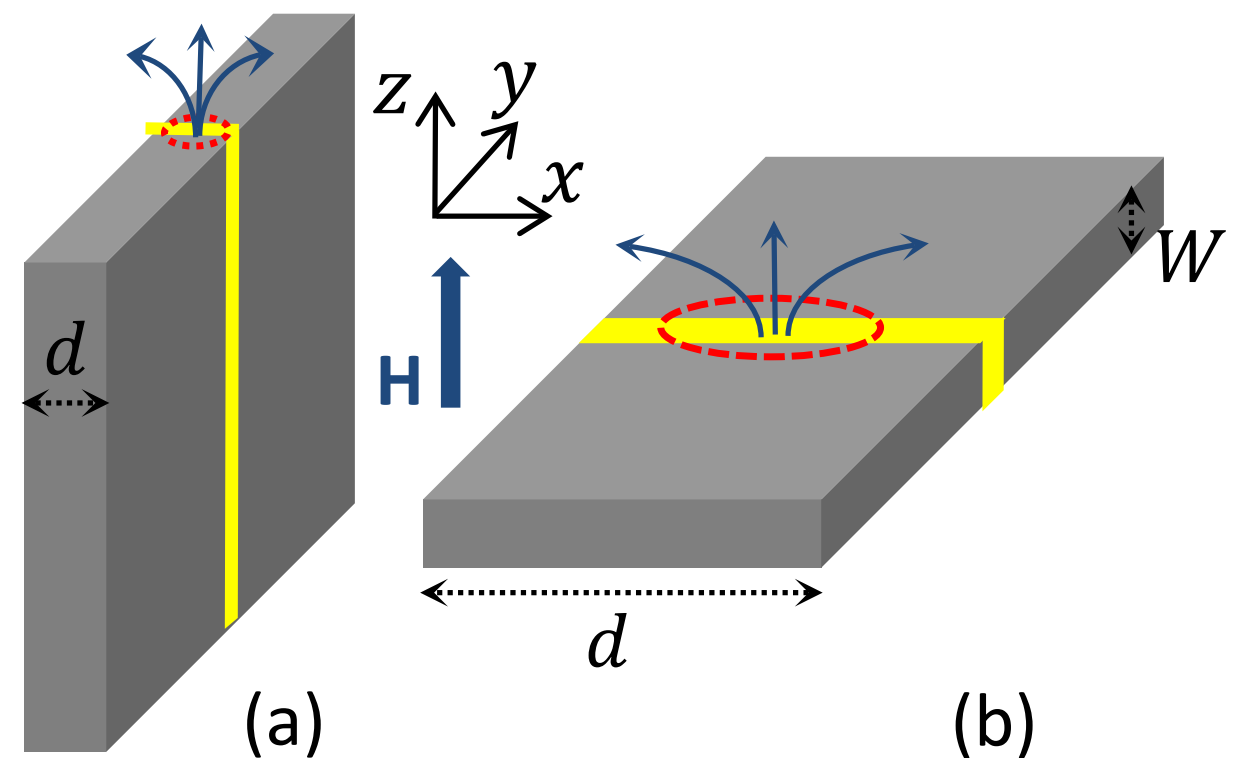

(b)

FIG. 51: Geometries of a Josephson junction in a thin film with the vortex (a) parallel and (b) perpendicular to the broad face of the film.

where $\mathbf{A}$ is the vector potential and $\varphi$ is the phase of the order parameter. The current continuity condition $\partial_{x} J_{x}+\partial_{y} J_{y}=0$ can be satisfied by expressing $J_{x}=\partial_{y} g$ and $J_{y}=$ $-\partial_{x} g$ in terms of a stream function $g(x, y, t)$. From (100), it follows that any nonuniform phase difference $\theta(x)=\varphi(x,+0)-\varphi(x,-0)$ on the junction results in a discontinuity of $J_{x}(x,+0)-J_{x}(x,-0)=\left(\Phi_{0} / 2 \pi \mu_{0} \lambda^{2}\right) \partial_{x} \theta(x)$, and a jump of the normal derivative in the stream function at $y=0$ :

$$
\left.\frac{\partial g(x, y)}{\partial y}\right|_{y=+0}-\left.\frac{\partial g(x, y)}{\partial y}\right|_{y=-0}=\frac{\Phi_{0}}{2 \pi \mu_{0} \lambda^{2}} \frac{\partial \theta}{\partial x} .
$$

Excluding $\varphi$ from (100) and (101) yields

$$
\nabla^{2} g-\frac{H}{\mu_{0} \lambda^{2}}=\frac{\Phi_{0}}{2 \pi \mu_{0} \lambda^{2}} \frac{\partial \theta}{\partial x} \delta(y),
$$

where $\delta(y)$ provides the boundary condition (102), and $H=\nabla_{z} \times \mathbf{A}$ is the $z$ component of the magnetic field. For a parallel vortex in a thin film shown in Fig. 51 a, we have $g=H$ and (103) yields the London equation for $H(x, y)$. For a perpendicular vortex in an edge junction, $H(x, y)$ in (103) is expressed in terms of $g(x, y)$ using the Biot-Savart law, which turns (103) into an integro-differential equation. The nonuniform equation in (103) can be solved using the Green function which is nothing but the solution of the London equation for either a parallel Abrikosov (A) vortex [134] or a perpendicular Pearl vortex [92] for the cases 
shown in Figs. 51 a and b, respectively. A general solution for $g(x, y)$ is rather cumbersome, so we consider simpler cases of a thin film with $d<\lambda$ and a bridge with $d<\lambda_{P}$ for which self-field effects and the London screening are inessential. Then $\mathbf{A}$ and $H$ in (100), (101) and (103) can be neglected, bias current density $J$ is uniform across the film, and (103) reduces to the Poisson equation for both geometries shown in Fig. 51:

$$
\nabla^{2} g=\frac{\Phi_{0}}{2 \pi \mu_{0} \lambda^{2}} \frac{\partial \theta}{\partial x} \delta(y)
$$

Setting $x=0$ in the middle of the film, we obtain the following solution for $g(x, y)[134]$ which satisfies the boundary condition of $J_{x}( \pm d / 2,0)=\partial_{y} g( \pm d / 2, y)=0$ at the junction edges:

$$
g(x, y)=-J x-\frac{\Phi_{0}}{8 \pi^{2} \mu_{0} \lambda^{2}} \int_{-d / 2}^{d / 2} \ln \frac{\cosh \frac{\pi y}{d}+\cos \frac{\pi}{d}(x+u)}{\cosh \frac{\pi y}{d}-\cos \frac{\pi}{d}(x-u)} \frac{\partial \theta}{\partial u} d u
$$

Using (105), the current density $J_{y}(x)=-\partial_{x} g(x, 0)$ through the junction is calculated. Equating $J_{y}(x, 0)$ to the sum of Josephson, resistive, and displacement current densities, and integrating by parts as shown in Appendix A, we obtain the following equation for $\theta(x, t)$ :

$$
\begin{gathered}
\ddot{\theta}+\eta \dot{\theta}+\sin \theta-\beta=\epsilon \int_{-1 / 2}^{1 / 2} \ln \left|\frac{2}{\sin \pi x-\sin \pi u}\right| \theta^{\prime \prime}(u) d u \\
\epsilon=\frac{l_{J}}{\pi d}=\frac{\Phi_{0}}{4 \pi^{2} \mu_{0} \lambda^{2} d J_{c}}
\end{gathered}
$$

where $x$ and $u$ are expressed in units of $d$, and the prime denotes differentiation with respect to the dimensionless coordinate $x$ along the junction. If the geometry-dependent screening effects caused by the vector-potential $\mathbf{A}$ in (100) and (101) are negligible, $\theta(x, t)$ is described by (106) for both cases shown in Fig. 51. We will use (106) for the calculations of vortices in Josephson junctions, and the average ohmic power $\bar{P}$ dissipated per unit height of the junction:

$$
\bar{P}=\frac{\eta P_{0}}{T} \int_{0}^{T} d t \int_{-1 / 2}^{1 / 2} \dot{\theta}^{2}(x, t) d x,
$$

where $P_{0}=\Phi_{0} J_{c} \omega_{J} d / 2 \pi$. Equations (44) and (106) take into account only ohmic losses but disregard radiation from a thin film junction into free space. The radiation losses are negligible due to a big mismatch of impedances of a superconductor and vacuum $[135,136]$, except for the extreme case of underdamped junctions with $\eta \ll 1$. In this chapter we calculate dynamics of vortices in overdamped and moderately underdamped junctions with 
$\eta>0.2$ for which the effect of radiation to free space on the power $\bar{P}$ and $\theta(x, t)$ in (106) is negligible.

To have a rough idea of the amount of power dissipated in a typical junction/GB, we can estimate the parameter $P_{0}$ for a junction of height $0.1 \mu \mathrm{m}$ using the GB characteristics mentioned in section3.3. The calculation results in $P_{0} \sim 61 \mathrm{nWatt}$ and $V_{0}=\Phi_{0} \omega_{J} / 2 \pi \sim$ $40 \mathrm{mV}$.

\subsection{CURRENT}

In this section we address dynamics of vortices described by the full equation in (106) for a dc bias current. We consider three situations: 1. Vortices penetrate from the edge of the junction where $J(x)$ exceeds $J_{c}$ due to a small gradient in $J(x)$ along the junction. 2. Vortices appear inside the junction in a region where $J_{c}(x)$ is locally reduced. 3. Vortices appear due to coexistence of current gradient and a defect in the junction. Most of the simulations of vortices presented below were done for $\epsilon=l_{J} / \pi d=2.10^{-3}$, that is, for the junctions much longer than the static AJ core size $l_{J}$. As will be shown later, for larger values of $\epsilon$ the size of the vortex becomes comparable to the length of the junction and a transition to phase slip will occur much sooner at lower currents.

We start with a static vortex for which (106) at $\beta=0$ was solved numerically with the initial distribution of $\theta(x, 0)=4 \tan ^{-1} \exp (-x / \epsilon)$ centered in the middle of the junction. To stabilize the vortex against attraction to the edges, a weak "pinning" potential modeled by $J_{c}(x)=\left[1-\delta \exp \left(-x^{2} / \zeta^{2}\right)\right] J_{c}$ was incorporated. Simulations of (106) in which $\sin \theta$ is replaced with $\left[1-\delta \exp \left(-x^{2} / \zeta^{2}\right)\right] \sin \theta$ and $\delta=\zeta=0.02$ show that the initial distribution $\theta(x, 0)$ evolves into stationary phase profiles presented in Fig. 52 for different values of $\epsilon$. As follows from Fig. 52 the vortex expands as $\epsilon$ increases. Eventually, the vortex spreads over the entire junction at $\epsilon>0.05$, and the static vortex-to-phase slip transition occurs at $\epsilon_{c}=1 / \pi^{2} \approx 0.1[137]$.

Unlike vortices in a long junction which are $2 \pi$ phase kinks with $\Delta \theta=\theta(\infty)-\theta(-\infty)=$ $2 \pi$, the AJ vortices in a short junction are partial phase kinks with $\Delta \theta<2 \pi$. The latter reflects the fact that the AJ vortex in short junctions carries a reduced magnetic flux $\phi<\phi_{0}$, as is characteristic of vortices in thin films [134]. Indeed, the fluxoid quantization [5, 42, 43] requires that:

$$
\Delta \theta+\frac{4 \pi \mu_{0} \lambda^{2}}{\Phi_{0}} \oint \mathbf{J} \cdot d \mathbf{s}=2 \pi,
$$

where the integral is taken along an infinite contour which encompasses the film surface. Because the vortex shown in Fig. 52 causes nonzero $\mathbf{J}(x, y)$ along the film edges at $x=-1 / 2$ 


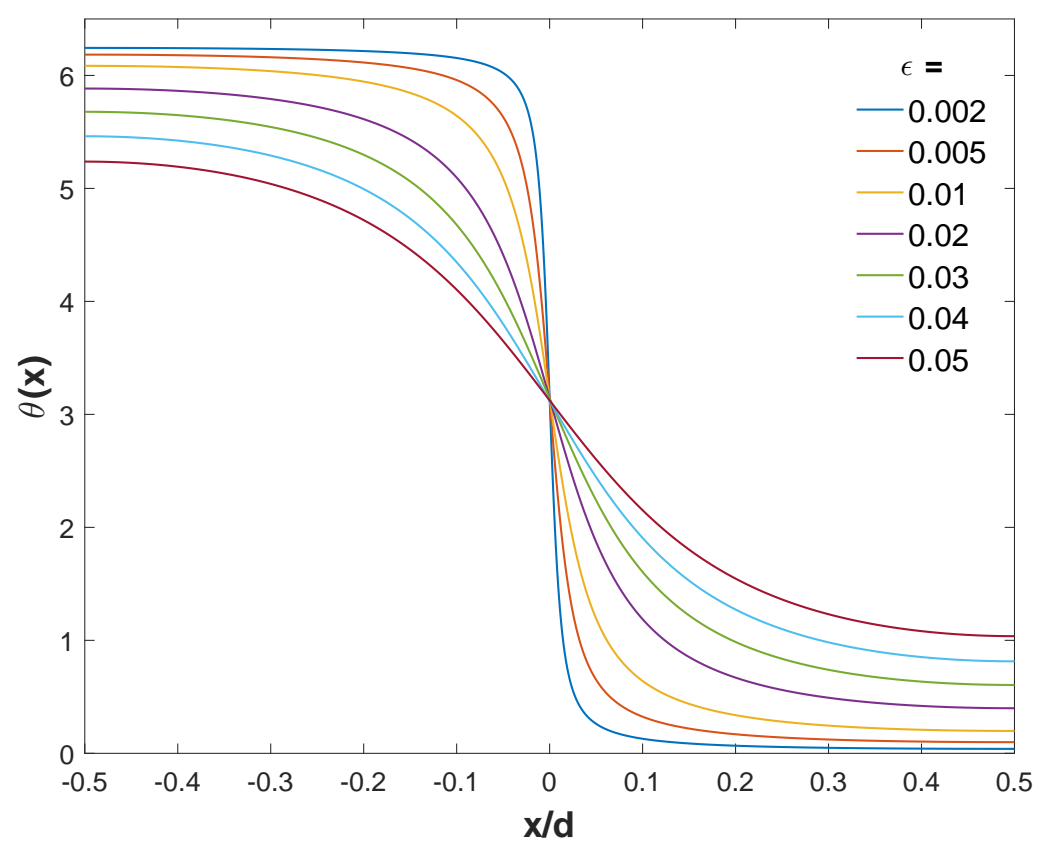

FIG. 52: $\theta(x)$ in a static vortex calculated from (106) for different values of $\epsilon$ as described in the text.

and $x=1 / 2,(109)$ yields $\Delta \theta<2 \pi$. A phase shift produced by a real A vortex on a junction in a thin film strip was observed in Ref. [138] and calculated in Ref. [139].

\subsubsection{JUNCTION WITH WEAK SCREENING}

Consider penetration of vortices in the junction, assuming that $\beta(x)=(1-k x) \beta_{0}$ in (106) has a small gradient with $k \ll 1$. The slight inhomogeneity in $J(x)$ with $k=d / \Lambda,(\Lambda$ being the magnetic penetration depth for each geometry) can result from self-field effects of transport current or a dc field applied to one side of a thin film screen with a perpendicular Josephson junction shown in Fig. 51. It turns out that the dynamic behavior of vortices in overdamped $(\eta \gtrsim 1)$ and undedramped $(\eta \lesssim 1)$ junctions is markedly different. For an overdamped junction, simulations of (106) with $\beta(x)=(1-k x) \beta_{0}$ show that, once $J$ exceeds $J_{c} /(1+k)$, vortices start penetrating one by one through the left edge of the junction and exiting from the other end (Fig. 53). As the current increases, the flight time of vortices through the junction decreases and the size of a vortex increases, as shown in Fig. 54. The expansion of moving $\mathrm{J}$ and AJ vortices as $\beta_{0}$ increases is characteristic of the overdamped limit $[43,76,94]$, as illustrated by (81) and (82). Based on the expansion of vortices with current, we can therefore expect a dynamic transition of the moving vortex into a phase slip 
as the current increases, even if the static vortex can exist in a large junction.

Our numerical simulations of (106) with $\eta \gtrsim 1$ have shown that a gradual transition of a moving vortex into a phase slip does happen as $\beta_{0}$ increases and the vortex spreads over the entire junction. In this case $\theta(x, t)$ becomes flat and increases nearly linearly with $t$. For $\beta_{0} \gg 1$, the phase slip state $\theta(x, t)$ is described by

$$
\theta(x, t)=\theta_{0}(t)+\delta \theta(x, t)
$$

where $\theta_{0}(t)$ satisfies the equation for a point contact:

$$
\ddot{\theta}_{0}+\eta \dot{\theta}_{0}+\sin \theta_{0}=\beta_{0} .
$$

For $\beta_{0} \gg 1$ and $\eta \gg 1$, an approximate solution of (111) is:

$$
\theta_{0}(t)=\frac{\beta_{0} t}{\eta}+\frac{\eta^{2}}{\beta_{0}^{2}+\eta^{4}}\left[\sin \frac{\beta_{0} t}{\eta}+\frac{\eta}{\beta_{0}} \cos \frac{\beta_{0} t}{\eta}\right]
$$

The small correction $\delta \theta(x, t)$ results from the integral and the nonlinear terms in (106). Figure 55 shows that $\delta \theta(x, t)$ oscillates around a stationary profile $\theta_{s}(x)$ which comes from the weak inhomogeneity of $\beta(x)=(1-k x) \beta_{0}$ (see Appendix A):

$$
\theta_{s}(x)=-\frac{4 k \beta_{0}}{\pi^{4} \epsilon} \sum_{n=o}^{\infty} \frac{(-1)^{n} \sin \pi(2 n+1) x}{(2 n+1)^{3}}
$$

To see how the gradual transition from the vortex to the phase slip state can manifest itself in the $V-I$ characteristics, we calculated the averaged instantaneous voltage on the junction:

$$
V(t)=\frac{\Phi_{0} \omega_{J}}{2 \pi} \int_{-1 / 2}^{1 / 2} \dot{\theta}(x, t) d x=\sum_{\omega} V_{\omega} \exp (i \omega t) .
$$

Here $V(t)$ has multiple Fourier harmonics caused by superposition of Josephson oscillations and motion of vortices. The behavior of AJ vortices in a long junction can be inferred from the dc component of voltage $\bar{V}\left(\beta_{0}\right)$ shown in Fig. 56. At $\eta=2$ the calculated $V-I$ curve follows $V=I_{c} R \sqrt{\beta_{0}^{2}-1}$ for the overdamped point junction [42] for all $\beta_{0}$ except for a vicinity of $\beta_{0} \approx 1$ where the phase slip transition occurs. At $\eta \lesssim 1$ the $V-I$ curves acquire stepwise features and become hysteretic. Here the jumps in the ascending branches of $\bar{V}\left(\beta_{0}\right)$ result from penetration of several vortices which then turn into a phase slip state at larger $\beta_{0}$ indicated by the dashed arrows. The descending branches of $\bar{V}\left(\beta_{0}\right)$ exhibit staircase structures where steps correspond to different numbers of vortices indicated by vertical arrows. 


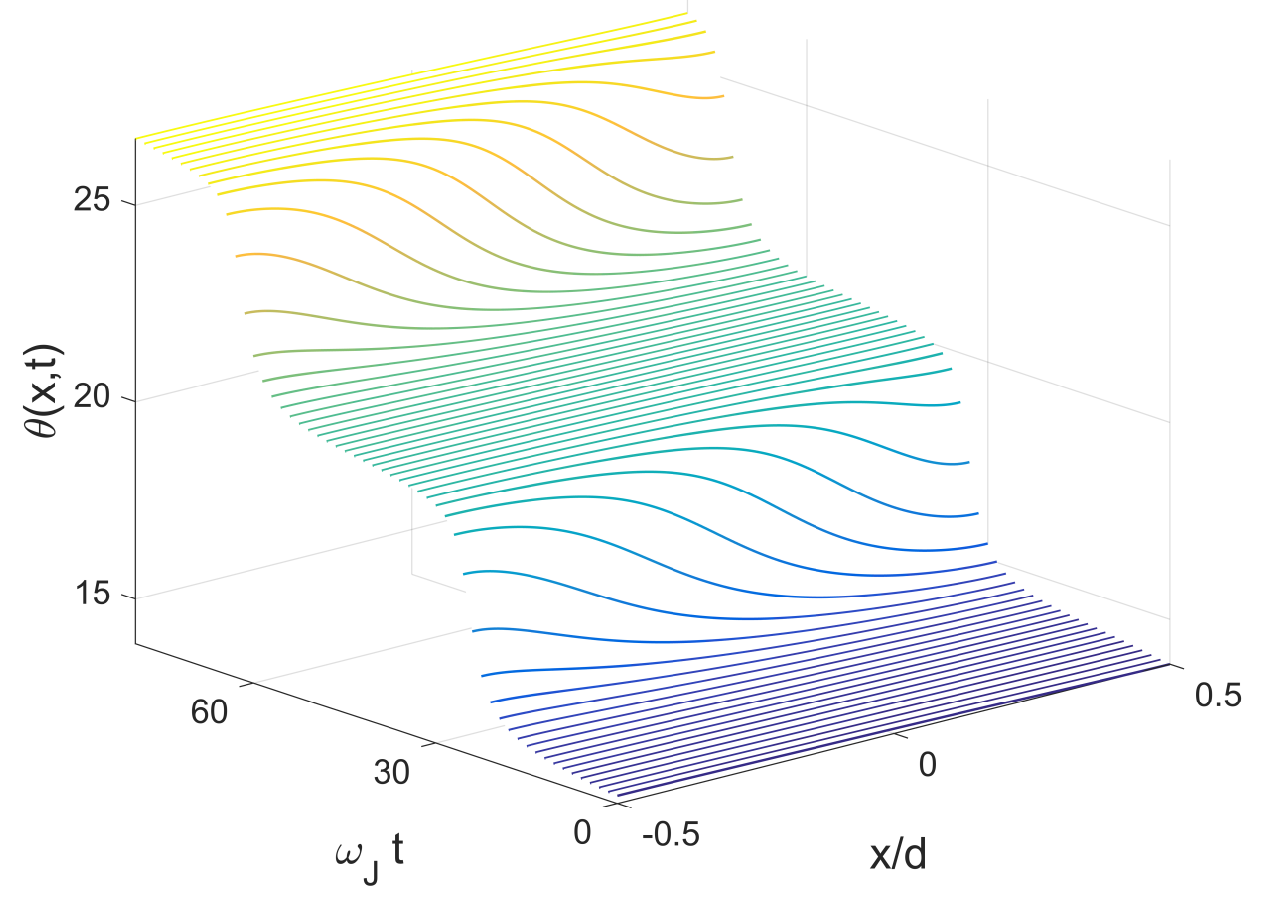

FIG. 53: Penetration of single vortices in an overdamped junction with $\eta=2$ and $\beta_{0}=1.05$ calculated for $k=0.02$ and $\epsilon=2 \cdot 10^{-3}$.

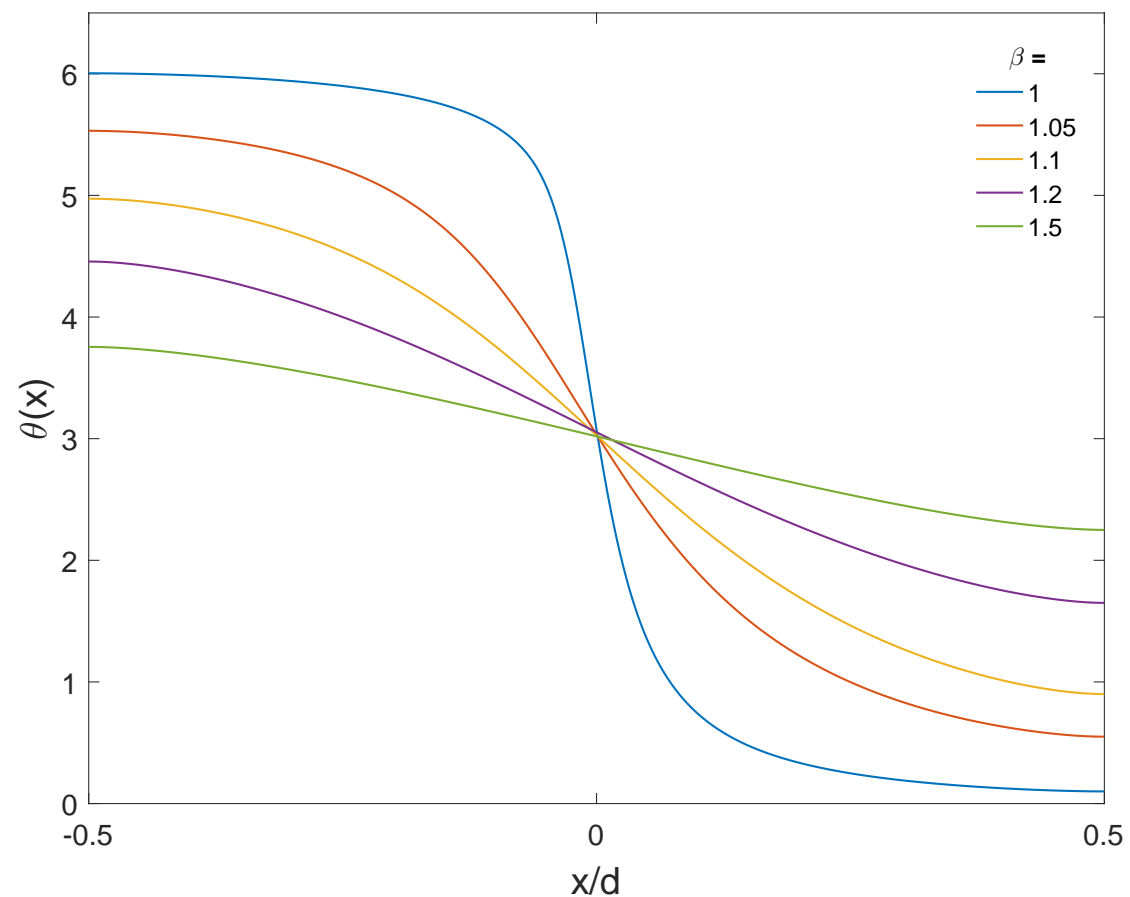

FIG. 54: Snapshots of moving vortices in the middle of the junction calculated from (106) for different currents at $\eta=2, k=0.02$ and $\epsilon=2 \cdot 10^{-3}$. 


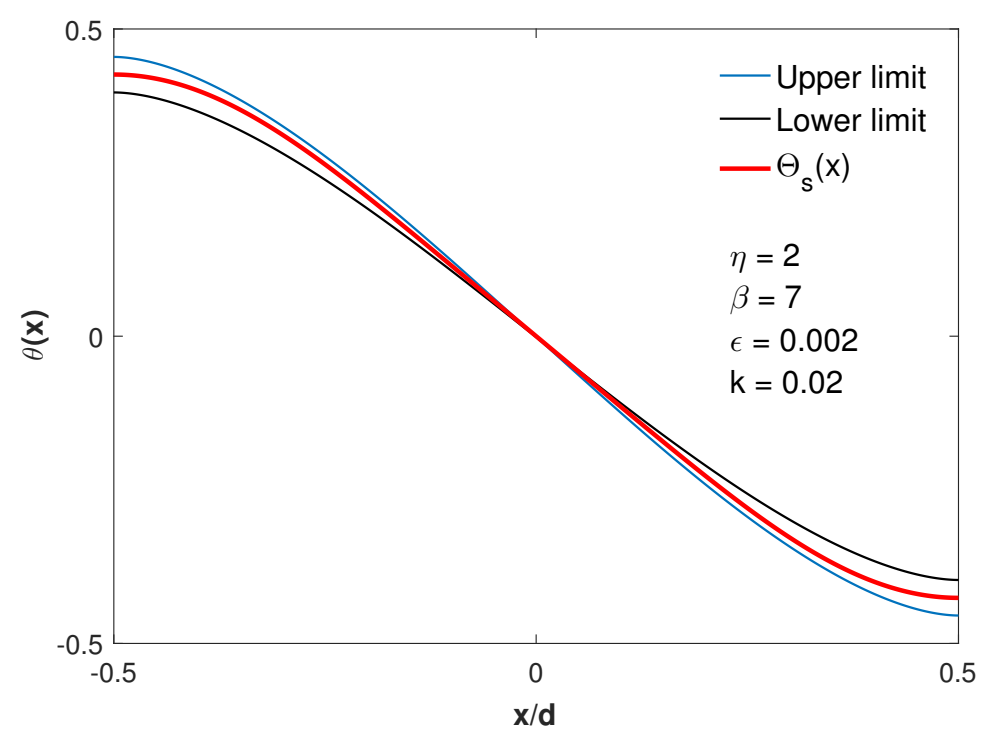

FIG. 55: Upper and lower limits between which $\theta(x, t)$ oscillates, calculated for $\eta=2$ and $\beta_{0}=7$. The red curve shows $\theta_{s}(x)$ described by (113).

The behavior of $\bar{V}\left(\beta_{0}\right)$ on the ascending branch is illustrated by Figs. $53-54$ and 57-59 which show representative $\theta(x, t)$ calculated for different values of $\eta$ and $\beta_{0}$. In an overdamped junction $(\eta \gtrsim 2)$ vortices periodically appear at the left edge, move along the junction and disappear at the right edge. As $\beta_{0}$ increases vortices move faster and become longer, which eventually results in the transition to the phase slip state described above (see Fig. 53). In this case strong ohmic dissipation suppresses both the Cherenkov radiation caused by the nonlocal effects and bremsstrahlung resulting from acceleration and deceleration of a vortex as it moves along the junction. This behavior of vortices starts changing at $\eta \approx 1$ as the radiation wake behind a moving vortex shown in Fig. 57 becomes apparent. In this case vortices which reach the edge of the junction get reflected as vortices of opposite polarity (antivortices). As a result, vortices penetrating from the left edge of the junction collide with antivortices reflected from its right edge; at $\eta \lesssim 1$ these vortices and antivortices do not annihilate but go through each other, similar to underdamped Josephson vortices described by the sine-Gordon equation [42]. As current further increases, the number of vortices and antivortices in the junction increases which corresponds to the steps shown in Fig. 56. Eventually counter-moving vortices and antivortices form a dynamic pattern shown in Fig. 58. This state can be regarded as a nonlinear wave on the background phase $\theta_{0}(t)$ which increases with time, so that the snapshots of the dynamic structure shown in Fig. 58 shift up and periodically replicate themselves. As current increases, the overlap of vortices 

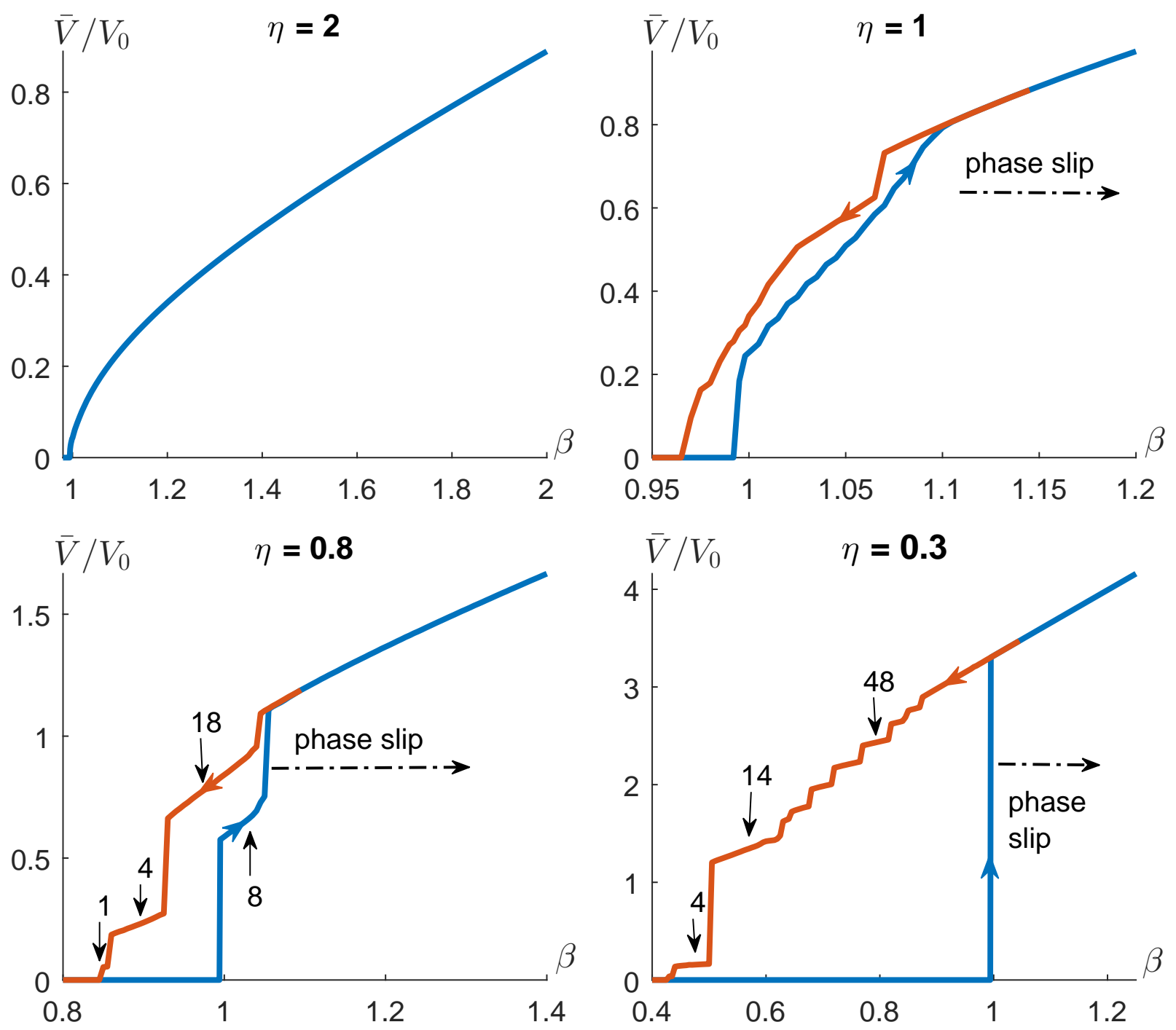

FIG. 56: The de voltage $\bar{V}=\langle V(t)\rangle$ calculated from (114) for different values of $\eta$, where $\langle\ldots\rangle$ denotes time averaging, and $V_{0}=\Phi_{0} \omega_{J} / 2 \pi$. 


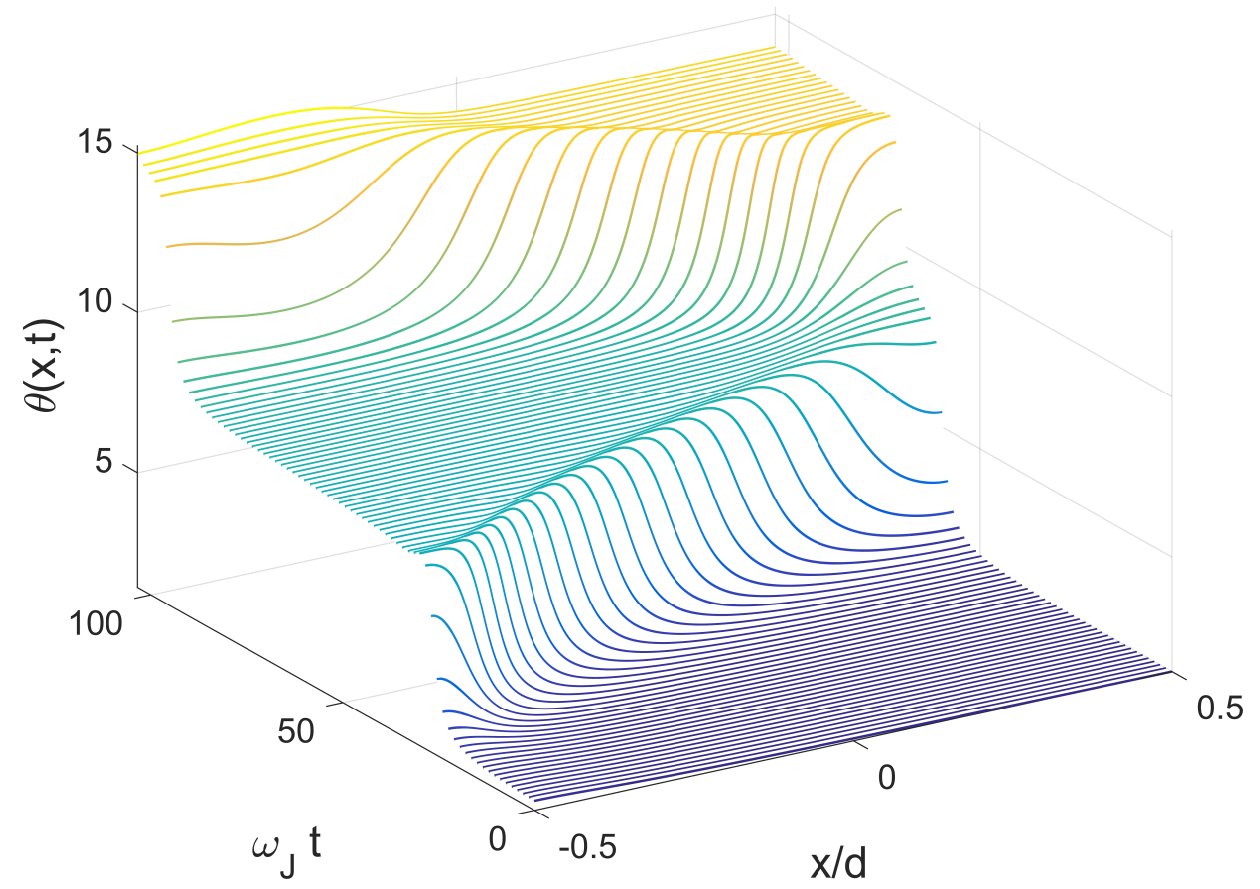

FIG. 57: A wake radiated behind the moving vortex at $\eta=1$ and $\beta_{0}=0.995$. Here the vortex gets fully reflected from the edge as antivortex.

and antivortices reduces the amplitudes of the phase waves as shown in Fig. 58b. With further increase in current, this structure which manifests itself in the behavior of $\bar{V}\left(\beta_{0}\right)$ at $1<\beta_{0}<1.15$, turns into a phase slip state as shown in Fig. 56.

At $\eta=0.9$ the first signs of vortex splitting instability caused by the Cherenkov wake behind the vortex penetrating from the left edge appear. As the vortex approaches the right edge it accelerates due to attraction to the edge so that the wake amplitude increases and exceeds a critical value above which a v-av pair forms. The junction eventually goes into a dynamic steady-state after two more v-av pairs are generated at the edges. Further increase in current causes penetration and production of more vortices similar to Fig. 58. The Cherenkov instability becomes more apparent at $\eta=0.8$ for which the wake amplitude exceeds the threshold when the vortex reaches the middle of the junction where a v-av pair first appears. The newborn vortex and antivortex move apart, accelerate and produce another v-av pair. These vortices with opposite polarities oscillate back and forth in the junction and form a dynamic structure similar to that is shown in Fig. 58. At a slightly higher current more v-av pairs are generated and the junction goes into the phase slip state. Figure 59 shows the immediate instability of a left penetrating vortex at the threshold current 

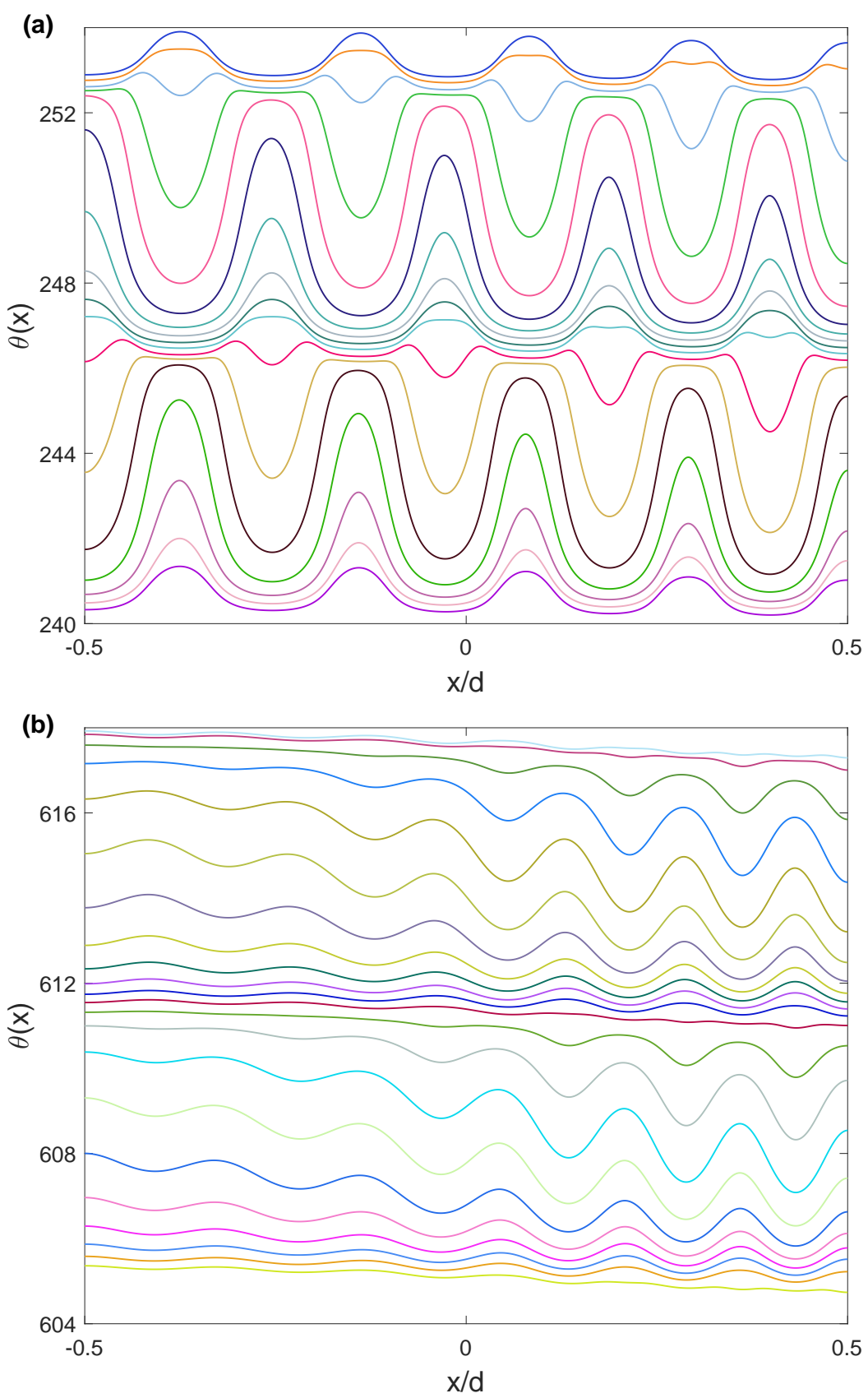

FIG. 58: Snapshots of dynamic patterns formed by counter-moving vortices and antivortices calculated for $\eta=1, \epsilon=2 \cdot 10^{-3}, k=0.02$, (a) $\beta_{0}=1.05$ and (b) $\beta_{0}=1.09$. Different colors correspond to different times $t$ during the time period after which the phase structures repeat themselves periodically after shifting up in $\theta$. As current further increases, the patterns shown in Figs. 58 a and b gradually turn into a phase slip profile similar to that is shown in Fig. 55. The asymmetry of the profiles $\theta(x, t)$ with respect to $x=0$ is due to the effect of the gradient in $\beta(x)$. 
of $\beta_{0}=0.995$ calculated for $\eta=0.7$.

At $0.3<\eta<1$ dynamic multi-vortex structures on the ascending branch of $\bar{V}\left(\beta_{0}\right)$ exist in a narrow range of currents $(1+k)^{-1}<\beta_{0}<\beta_{p s}$ ( $\beta_{p s}$ : phase slip current) which shrinks as $\eta$ decreases and vanishes at $\eta \approx 0.3$. Vortices at $\eta<0.3$ exist only during a transient period during which the junction goes into a phase slip state after the current density at the edge reaches the threshold of vortex penetration. For instance, our simulations of (106) at $\eta=0.2$ indicates that, once a vortex enters the junction, it starts to become unstable and produce v-av pairs right at the edge. The cascade of v-av pairs displays itself as a chain of penetrating vortices from left edge which soon drives the junction into a resistive phase slip state. This behavior is similar to the phase pile expansion shown in Fig. 40. Simulation videos of the dynamics of the junctions in different regimes are available at Ref.[140]. The Cherenkov instability of vortex right after it enters through the edge of the junction and the subsequent transition to resistive state manifests itself in the big jump on the ascending branches of $\bar{V}\left(\beta_{0}\right)$ shown in Fig. 56 for $\eta=0.3$. However, the subsequent decrease of current results in re-appearance of vortices from phase slip state, which manifests itself in the hysteresis in the $V-I$ curves and the staircase form of the descending branch of $\bar{V}\left(\beta_{0}\right)$. The evolution of non-hysteretic $V-I$ curves to hysteretic ones upon decreasing $\eta$ in a long junction considered here resembles the well-known transition from non-hysteretic to hysteretic $V-I$ curves in point junctions $[42,43]$, except that the returned descending branch of $\bar{V}\left(\beta_{0}\right)$ in Fig. 56 is controlled by vortices emerged from the phase slip state.

Shown in Fig. 60 is the power $\bar{P}\left(\beta_{0}\right)$ dissipated by moving vortices calculated from (106) and (108) for different $\eta$. The curves $\bar{P}\left(\beta_{0}\right)$ have jumps and hysteretic features at the onset of vortex penetration which reflect those in Fig. 56. However, once $\beta_{0}$ exceeds the phase slip transition threshold, the dependence of $\bar{P}$ on $\beta_{0}$ nearly follows that of a point junction and exhibits the ohmic quadratic behavior $\bar{P}=P_{0} \beta_{0}^{2} / \eta$ at large $\beta_{0}$. The latter is similar to $\bar{P}\left(\beta_{0}\right)$ for Josephson vortices in a long junction described by the sine-Gordon equation in section 3.2.

Transitions between different dynamic vortex patterns can also manifest themselves in the voltage Fourier spectrum in (114). We calculated the Fourier spectrum by solving (106) with a uniform current $\beta$ and $\eta=0.8$, using the static solution for $\epsilon=0.002$ shown in Fig.52 as the initial condition. It turned out that if $\beta<0.84$, the vortex is pushed by the current to the edge of the junction and exits. However at $\beta>0.85$, the vortex becomes more energetic and gets trapped in the junction as it starts bouncing back and forth between the edges radiating Cherenkov and bremsstrahlung waves. Then the current was incrementally 


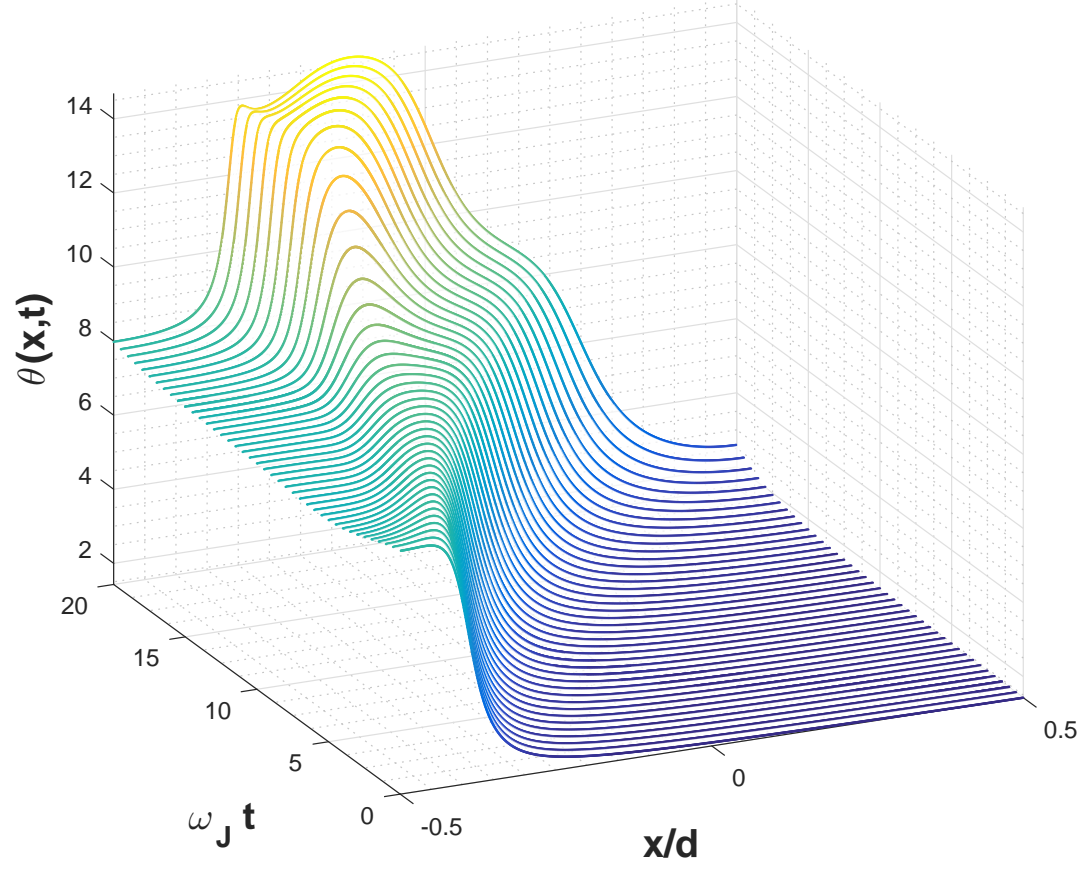

FIG. 59: Initial state of generation of v-av pairs calculated at $\eta=0.7$ and $\beta_{0}=0.995$. The Cherenkov splitting instability of a vortex occurs right after it enters the junction and ultimately results in the dynamic pattern similar to those shown in Fig. 58.

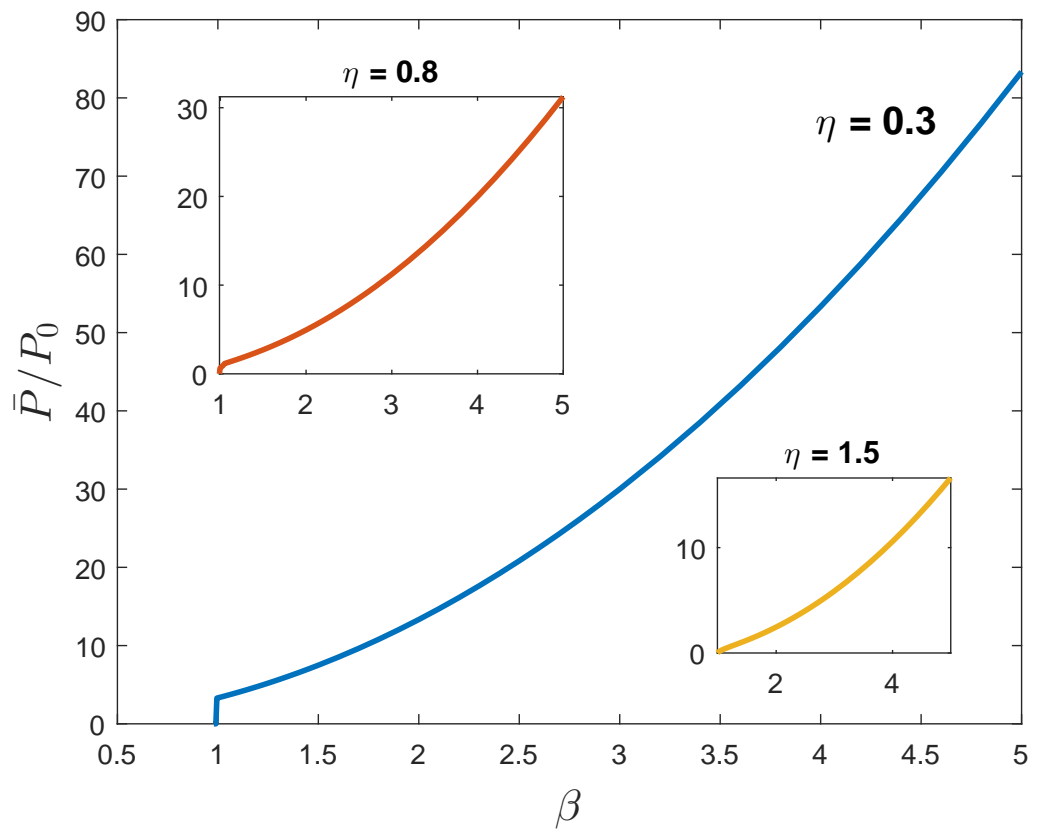

FIG. 60: Dissipation power vs de current calculated for different damping constants shows a quadratic behavior at currents well above the threshold of penetration of a vortex. 
increased to $\beta+\Delta \beta$ and (106) was solved using the calculated solution at the preceding $\beta$ as the initial condition. At some threshold current the amount of radiation is enough to make the vortex unstable and produce pair of v-av where the junction finally becomes stabilized, forming a periodically changing structures of vortices and antivortices glued by Cherenkov radiation, similar to those shown in Fig. 58. In this way the de voltage $\bar{V}(\beta)$ shown in the right panel of Fig. 61 was obtained. The so-calculated $\bar{V}(\beta)$ has jumps corresponding to the current-driven transitions between different number of vortices in the junction. Using the solution $\theta(x, t)$ we calculated the amplitudes $V_{\omega}$ of the Fourier harmonics

$$
V_{\omega}=\frac{V_{0}}{T}\left|\int_{0}^{T} d t e^{-i \omega t} \int_{-1 / 2}^{1 / 2} \dot{\theta}(x, t) d x\right|,
$$

where $T$ is the period of oscillations. The left panel of Fig. 61 shows the voltage Fourier spectra at different currents corresponding to different number of vortices in the junction. As the current increases and junction goes from a multi-vortex to the phase slip state, the amplitudes of low-frequency Fourier components $V_{\omega}$ with $\omega<\omega_{J}$ gradually diminish and finally disappear.

\subsubsection{PENETRATION OF VORTICES AT THE EDGE DEFECT}

Penetration of vortices in the junction can be facilitated not only by a weak gradient in $\beta(x)$, but also by a small defect at one of the edges. Such defects which are common in thin film junctions can locally reduce the Josephson critical current density $J_{c}(x)$. This situation can be modeled by (106) in which

$$
\begin{gathered}
\sin \theta \rightarrow[1-\delta(x)] \sin \theta, \\
\delta(x)=\delta_{0} \exp \left[-\frac{(x+1 / 2)^{2}}{\zeta^{2}}\right] .
\end{gathered}
$$

Here $\delta_{0}=\delta J_{c}(-d / 2) / J_{c}$ quantifies the magnitude of the local reduction of $J_{c}(x)$ at the edge, and $\zeta$ is the dimensionless length of the defect which can be of the order of the size of static vortex. Simulations regarding the size of the defect reveal that for a fixed current reduction of $\delta_{0}$, the size of the defect is inversely proportional to the amplitude of threshold current for vortex penetration. In other words, at fixed reduction of $\delta_{0}$, a larger defect would facilitate penetration of vortices at smaller currents.

The following results of simulations performed with $\zeta=0.05$ and $\delta_{0}=0.2$ assuming that $\beta$ is uniform show that vortices start to penetrate at $\beta \simeq 0.9$. At $\eta>1$ vortex penetration occur one by one, their size expands as current increases and the transition to the phase slip 

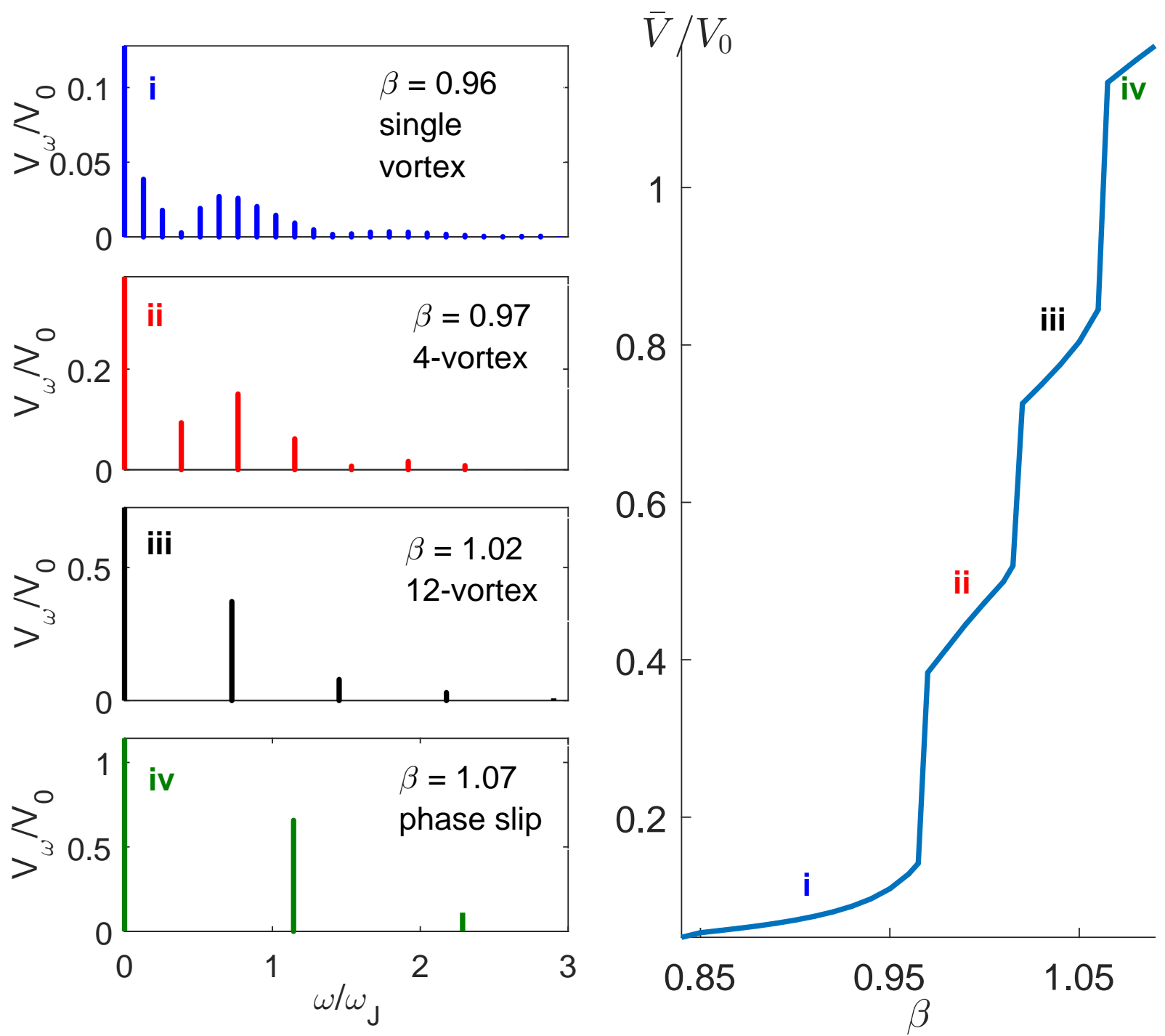

FIG. 61: Spectrum of Fourier components of voltage $V(t)$ calculated for $\eta=0.8$ and different currents corresponding to different number of vortices in the junction (left panel). Right panel shows the dc voltage $\bar{V}(\beta)$ in which jumps result from the change of the number of vortices in the junction. 
state occurs gradually at $\beta \gg 1$. At $0.7<\eta<1$ vortices get reflected from the edges and the radiation wake behind moving vortices becomes apparent. Further increase of $\beta$ yields dynamic structures similar to those shown in Fig. 58 and their subsequent transition to the phase slip state. At $\eta=0.7$ the radiation is strong enough to make a vortex unstable and produce a pair of $\mathrm{v}$-av at $\beta=0.95$. For values of $\eta<0.4$ a vortex depinned from the edge defect by strong threshold current accelerates and produces enough radiation to generate a v-av pair which then multiplies and drives the entire junction into the resistive phase slip state. Obviously, if the defect is stronger, meaning that the current reduction at the defect is large, vortices can penetrate and propagate in the junction at smaller threshold currents without radiating enough to become unstable. Nevertheless, if current increases the vortex becomes unstable and produces pairs. An example of such transient state is shown in Fig. 62 in which the first v-av pair appears as the initial vortex traveled more than half the length of the junction. More simulations of dynamic vortex structures in the presence of edge defects are available at Ref. [140]. The apparent similarity of the behavior of vortices for the cases of point defect and current gradient suggests that, no matter how a vortex enters the junction, the dynamics of vortices and the transition to the phase slip state are mostly controlled by the values of $\eta$ and $\beta$.

\subsubsection{INTERACTION OF VORTICES WITH PINNING CENTERS IN THE JUNCTION}

Consider now a moving vortex interacting with a defect in the middle of the junction in which case $\delta(x)$ in (115) and (116) is modeled by a Gaussian peak centered at $x=0$. Let a vortex enter from the left edge of the junction due to a weak current gradient $\beta(x)=$ $(1-k x) \beta_{0}$ with $k=0.1$, as was considered in section 5.2.1. We focus here on strong currents $\beta_{0} \gtrsim 0.9$ for which the defect is too weak to pin the vortex, yet the dynamics of vortices can change substantially, depending on the values of $\delta_{0}$ and $\zeta$. Shown in Fig. 63 are the results of simulations for a weak defect with $\delta_{0}=0.15$ and $\zeta=0.01$ at $\eta=1$. Here the vortex enters from the left edge of the junction, accelerates and decelerates as it approaches and passes the defect, and then accelerates again as it exits from the right edge. Dynamics of the vortex can change markedly if $\eta$ is reduced and the radiation effects become essential. For instance, in the case of $\eta=0.7$ shown in Fig. 64, the Cherenkov wake increases as the vortex accelerates toward the defect, the wake amplitude exceeds the critical value at which the vortex produces a v-av pair as it passes through the defect. At smaller $\eta$ the vortex penetrating from the edge starts generating v-av pairs before it reaches the defect, as was 


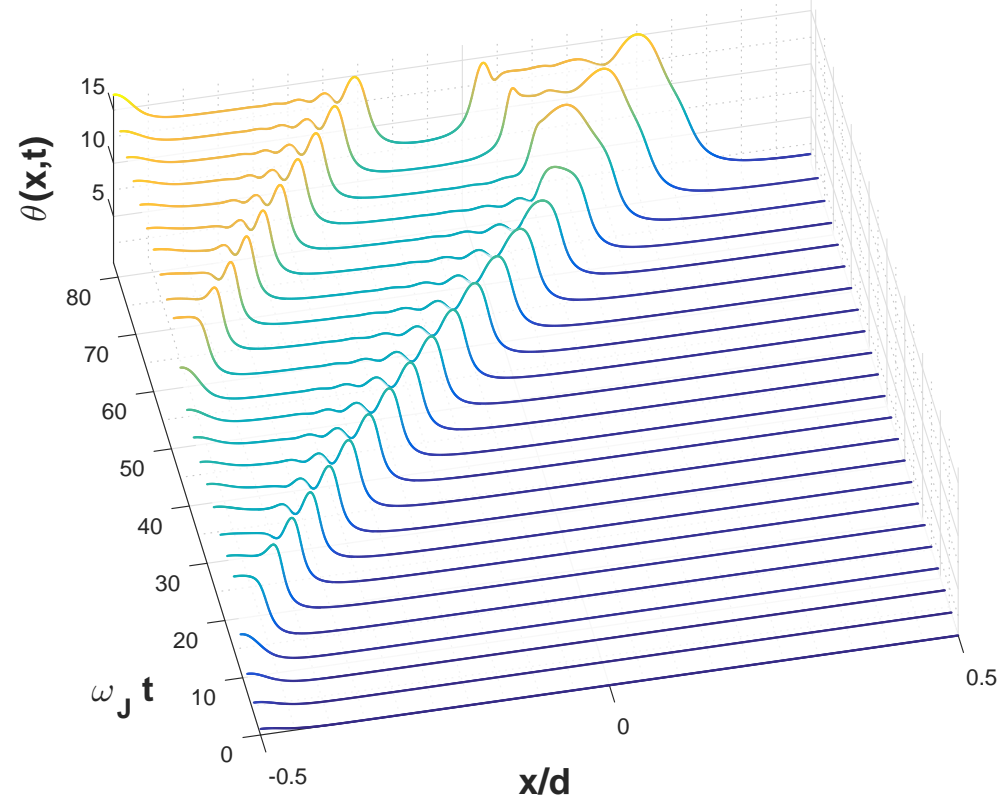

FIG. 62: A vortex depinned from the defect at the left edge of junction accelerates and produces a vortex-antivortex pair at $x \approx 0.1$ after the next vortex enters the junction. Simulations were done for $\delta_{0}=0.5, \beta=0.8$ and $\eta=0.3$.

described before.

For a slightly stronger defect with $\delta_{0}=0.2$ while screening $k=0.1$ is unchanged, v-av pair can appear at the defect simultaneous to a vortex penetration from left. The subsequent dynamics of this vortex state depends on the values of $\eta$ and $\beta_{0}$. For instance, at $\eta=1$ and $\beta_{0}=0.98$, the vortex penetrating from left annihilates with the antivortex produced at the defect in the middle of the junction, while the remaining vortex exits from the right edge, as shown in Fig. 65. However, for the same parameters at larger current $\beta_{0}=0.995$, vortex and antivortex go through each other. Defects with $\delta_{0}>0.2$ and $\zeta=0.01$ can trigger generation of $\mathrm{v}$-av pairs in the middle of the junction at a critical value $\beta_{0} \approx 1$ before any vortex enters from edges. In this case dynamics of vortices depends on $\eta$ in the same way as for the edge defect discussed in section 5.2.2. For a uniform current $(k=0)$, penetration of vortices at the edge defect can be mapped onto generation of v-av pairs at the bulk defect in the region $0<x<0.5$, the two cases become equivalent if the length of the junction for the edge defect is reduced by half, that is, the parameter $\epsilon$ is doubled.

\subsection{AC CURRENT}




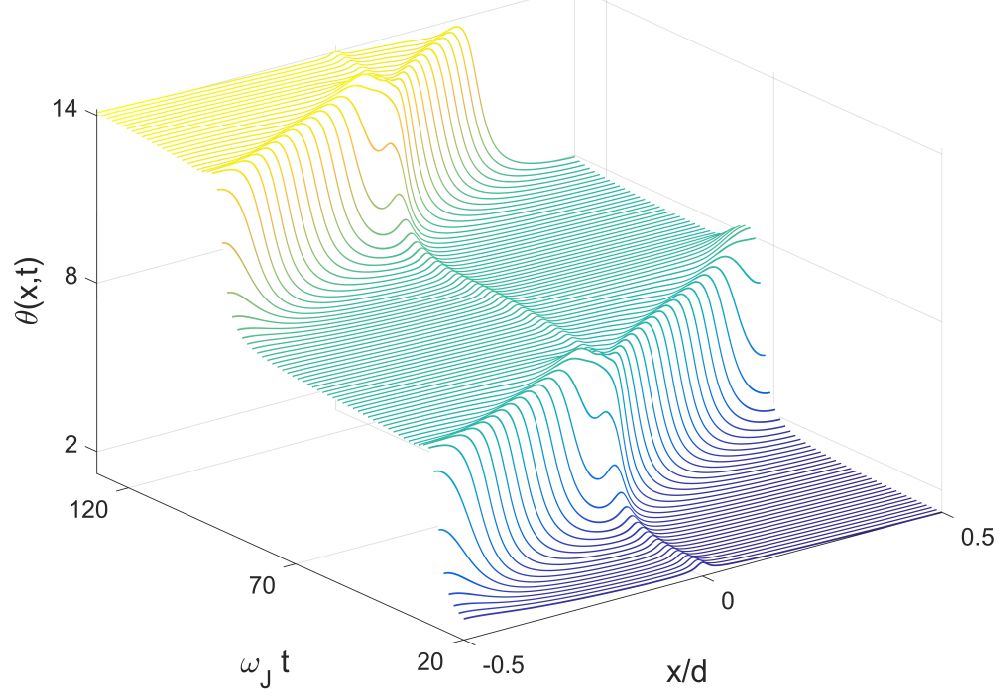

FIG. 63: A vortex accelerates as it approaches the defect in the center and decelerates once it passes the defect in the case of $\zeta=0.01, \delta_{0}=0.15, \beta_{0}=0.98$, and $\eta=1$.

Here we address dynamics of vortices under ac current with a small gradient in $\beta(x, t)=$ $\beta_{0}(1-k x) \sin \omega t$, where $\omega$ is the dimensionless frequency in units of $\omega_{J}$. The frequency of the current is chosen large enough so that it represents the alternating essence of the electromagnetic field and is computationally efficient. However, in most applications $\omega \ll \omega_{J}$. Therefore, results presented below were obtained by simulating (106) at $\omega=\pi / 30$. To be more experimentally relevant, a solution obtained at current $\beta_{0}-\delta \beta$ is used as initial condition for next calculation at $\beta_{0}$ while $\delta \beta \sim \mathcal{O}\left(10^{-3}\right)$.

Dynamics of $\theta(x, t)$ under ac current has several distinctive features as compared to the dc current:

1. Since $\beta(t)$ changes sign periodically, penetration of vortices from the left edge is followed by penetration of antivortices. Vortices and antivortices produced during positive and negative cycles of $\beta(t)$ collide and either annihilate or produce bursts of radiation inside the junction.

2. Vortices only penetrate during parts of the ac period when $\beta(t)=\beta_{0} \sin \omega t$ exceeds the penetration threshold $\beta_{c}$. Our results show that $\beta_{c}$ depends on both $\omega$ and $\eta$ : for instance, $\beta_{c}$ decreases from 1.22 at $\eta=2$ to 1.01 at $\eta=0.2$.

3. Acceleration and deceleration of vortices under ac current bring about one more source of radiation which contributes to the generation of v-av pairs. 

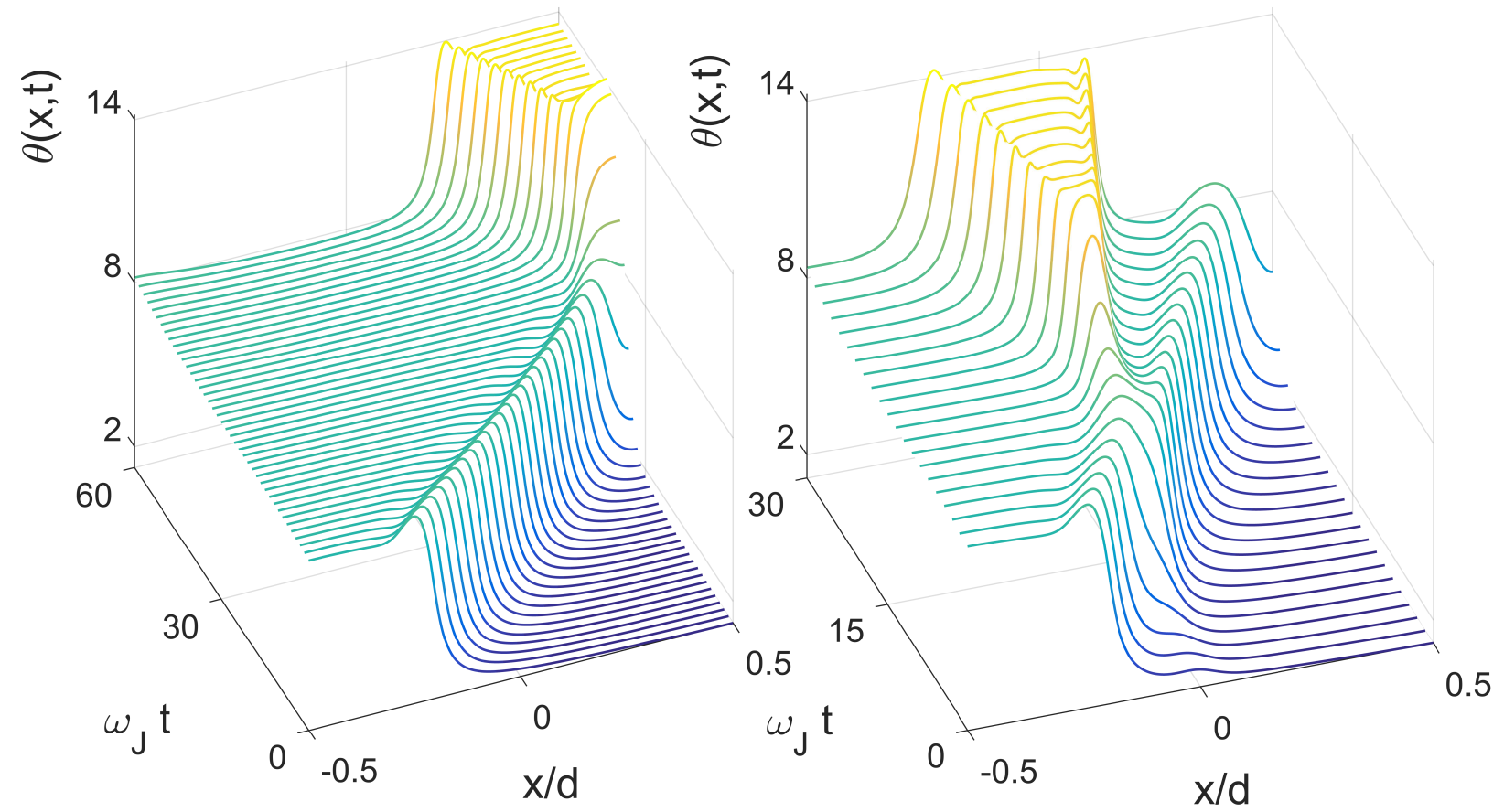

FIG. 64: At $\eta=0.7$ even a weak defect can accelerate the approaching vortex so that it produces a critical radiation wake which generates a v-av pair. Figure shows the dynamics of a vortex in the absence (left) and the presence (right) of a defect with $\delta_{0}=0.05, \zeta=0.05$ and $\beta_{0}=0.98$.

4. Dynamics of vortices under ac current changes markedly if the amplitude of oscillations of a vortex exceeds the length of the junction.

5. Resonance interaction of oscillating vortices with standing waves in the junction can significantly affect the transition from vortices to phase slips and the generation of v-av pairs. Analysis of these issues requires taking into account intertwined effects of $\eta, \omega$, $\beta_{0}$, and $d$ on the dynamics of $\theta(x, t)$.

6. Since the strong electromagnetic applied force is time-dependent, the steady state solution obtained may vary depending on the initial conditions used.

Given the complexity of the dynamics of vortices driven by the ac current and the multitude or different parameters involved, we only outline here a few essential cases (more details are available in Ref. [140]).

Figure 66 shows $\theta(x, t)$ calculated at $\eta=2$ at $\beta_{0}=1.237$ and $\omega=\pi / 30$. In this case a vortex enters the junction once $\beta(t)$ exceeds $\beta_{c}$ but, as $\beta(t)$ changes sign, the vortex turns around and exits through the same edge of the junction during the negative ac cycle, after 


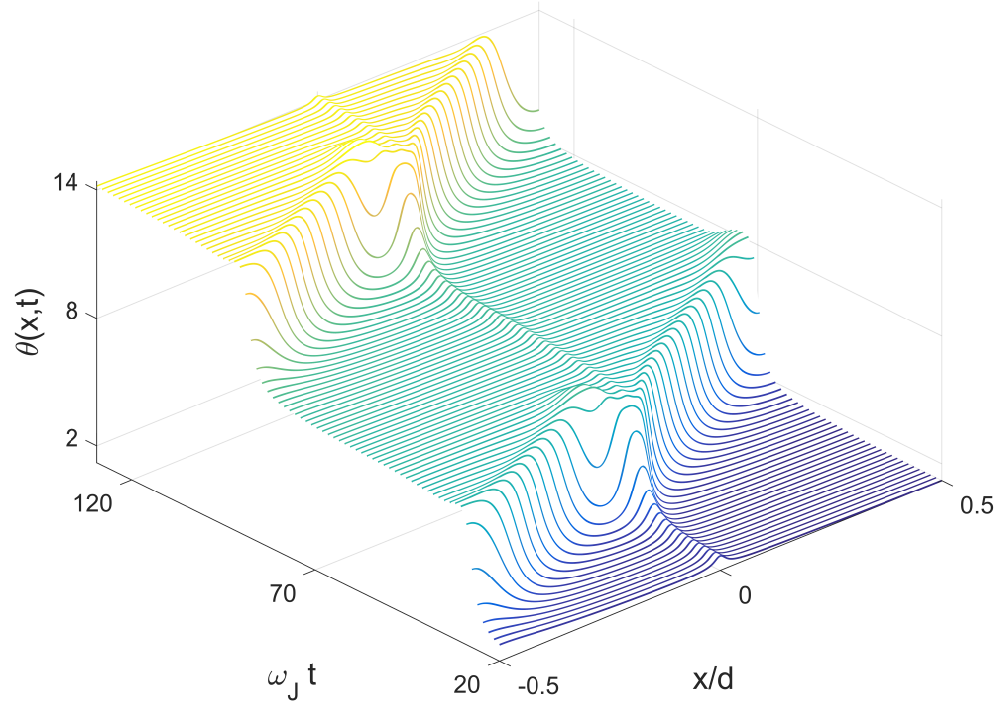

FIG. 65: Interaction of a vortex penetrating from left with a v-av pair produced simultaneously by a weak defect with $\delta_{0}=0.2$ and $\zeta=0.01, k=0.1$ and $\eta=1$ at the threshold current $\beta_{0}=0.98$. The vortex which entered from the left edge annihilates with the antivortex produced at the defect while the remaining vortex exits from the right edge.

which the whole process repeats. Neither antivortices nor radiation behind the moving vortex is visible here. However, at a slightly larger current $\beta_{0}=1.245$ the vortex expands further and becomes faster, so it can move all the way to the other end of the junction and exits before $\beta(t)$ changes sign. During the negative ac cycle the antivortex penetrates in the junction in the same way and extinguishes the positive $2 \pi$ phase shift left behind the preceding vortex, as shown in Fig. 67. The transition from the oscillating to the ballistic vortex dynamics manifests itself in the Fourier spectrum of voltage shown in Fig. 68. In the oscillatory state the Fourier spectrum consists of equidistant peaks at $\omega_{n}=n \omega$, where $\omega=\pi / 30$ and $n=1,2,3, \ldots$, while in the ballistic state the harmonics with even $n$ disappear. This transition also manifests itself in a negative jump in the dissipated power $\bar{P}\left(\beta_{0}\right)$ at $\beta_{0} \approx 1.245$, as shown in Fig. 69. Such $N$-shaped dependence of $\bar{P}\left(\beta_{0}\right)$ indicates a negative differential resistance and a hysteretic switching of the junction between two ascending branches of $\bar{P}\left(\beta_{0}\right)$ as the ac current amplitude is varied around $\beta_{0} \approx 1.245$. Here $\beta_{0} \approx 1.245$ appears to be only slightly below the value of $\beta_{0}$ above which the phase slip state emerges.

Behavior of vortices becomes more complex as $\eta$ is decreased. For instance, at $\eta=1$, the curve $\bar{P}\left(\beta_{0}\right)$ shown in Fig. 69 acquires a staircase shape, each step resulting from penetration of an additional vortex penetration. Close to the voltage onset at $\beta_{0}=1.102$ 
a vortex partially penetrates the junction during the positive cycle, then exits during the negative cycle, after which an anti-vortex partially enters and exits as the current changes sign again. This symmetry in v-av penetration breaks as current increases, so that a vortex penetrates deep into the junction during the positive ac cycle and returns during the negative ac cycle, prohibiting the antivortex from penetration, similar to the case shown in Fig. 66 for $\eta=2$. The breakdown of symmetry brings about a small feature in $V-I$ curve (hence power curve) shown in magnified inset of Fig. 69 at $\eta=1$. As current increases further the oscillating dynamics of a vortex becomes ballistic, resulting in a $N$-shaped feature in $\bar{P}\left(\beta_{0}\right)$ at $\beta_{0} \approx 1.118$. At $\beta_{0}>1.118$, the ballistic penetration of vortices and antivortices proceeds in the way similar to that is shown in Fig. 67 until the appearance of the next step on the $\bar{P}\left(\beta_{0}\right)$ curve which corresponds to penetration of an additional vortex. In this case the current amplitude is large enough so that one vortex moves ballistically along the junction followed by partial penetration of second vortex. As current changes to negative ac cycle, this second vortex returns from the same edge which is followed by ballistic penetration of an antivortex, extinguishing the $4 \pi$ phase shift during the positive cycle (Fig. 70). As the current increases further the transition from the oscillatory to ballistic dynamics of the second vortex also manifests itself in a small $N$-shaped feature in $\bar{P}\left(\beta_{0}\right)$ at $\beta_{0} \approx 1.269$ in Fig. 69. It turns out that, except for the small $N$-shaped features due to the transitions from oscillatory to ballistic dynamics of vortices, the curve $\bar{P}\left(\beta_{0}\right)$ calculated by solving Eq. (106) for $\eta=1$ is close to $\bar{P}\left(\beta_{0}\right)$ of a point Josephson junction.

Now we turn to smaller damping constants $0.3<\eta<0.7$ for which dynamics of vortices is essentially affected by their radiation field. For the case of $\eta=0.7$ shown in Fig. 69, the first jump on the ascending branch of $\bar{P}\left(\beta_{0}\right)$ at $\beta_{0}=1.068$ results from penetration of a vortex in the positive ac cycle and an antivortex in the negative ac cycle. However, as shown in Fig. 71 at $\beta=1.085$ the process of penetration and exit of vortices is drastically different from local models. As an example shown in Fig. 71a calculated at $\beta_{0}=1.085$, here when current exceeds the threshold of penetration, a vortex enters the junction and stops midway when current changes direction. As current goes through the negative cycle, the vortex feels a strong Lorentz force due to which the radiation wake behind the accelerating vortex exceeds the critical amplitude at which the vortex produces a v-av pair which however does not have enough time to expand and generate a cascade of v-av pairs because the ac current starts decreasing. The two vortices exit the junction right away while the antivortex moves in the opposite direction and stops midway in the junction. This process repeats for the antivortex during the positive ac cycle[140]. It is interesting to note that unlike local 


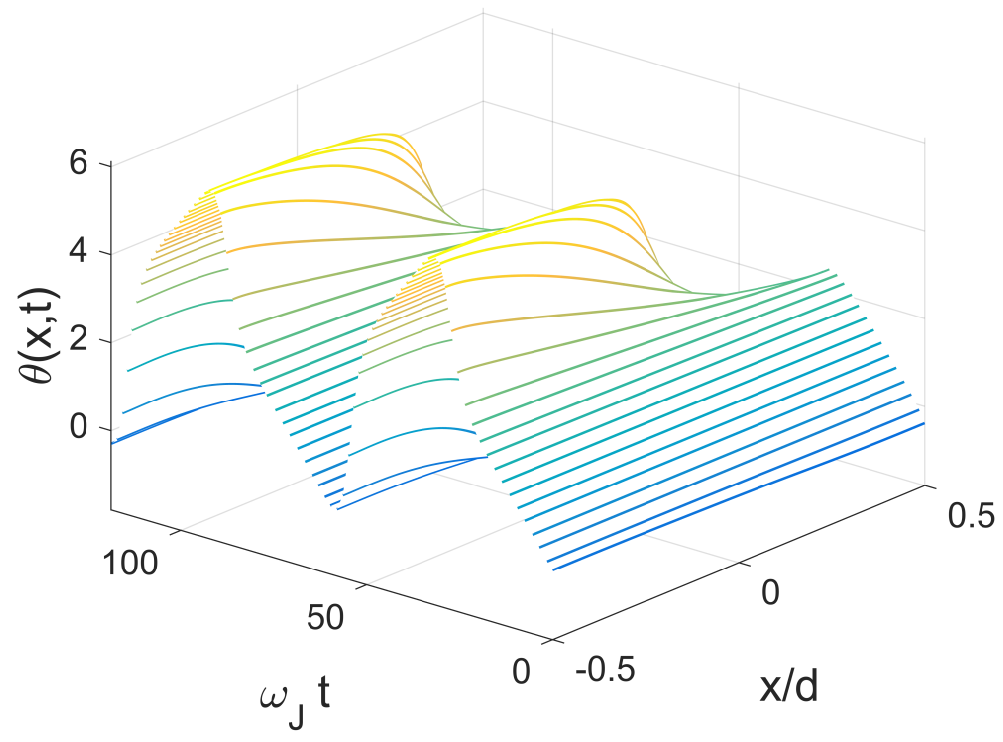

FIG. 66: Oscillatory dynamics of vortices in an overdamped junction with $\eta=2$ at the penetration threshold $\beta_{0}=1.237$. The vortex enters the junction during the positive cycle of $\beta(t)$, stops midway when $\beta(t)=0$, turns around and exits through the edge during the negative cycle of $\beta(t)$.

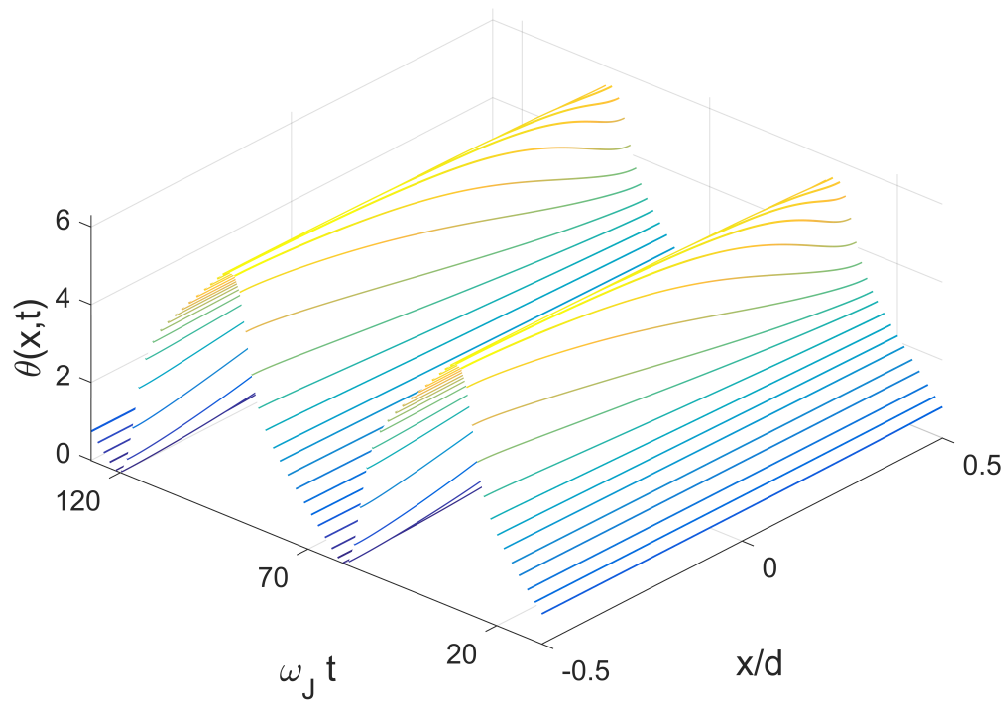

FIG. 67: Ballistic penetration of vortices and antivortices into an overdamped junction with $\eta=2$ at $\beta_{0}=1.245$. Here vortices and antivortices traverse the junction and exit from the other end. Notice that the moving vortex extends nearly over the entire junction and produces no visible radiation. 


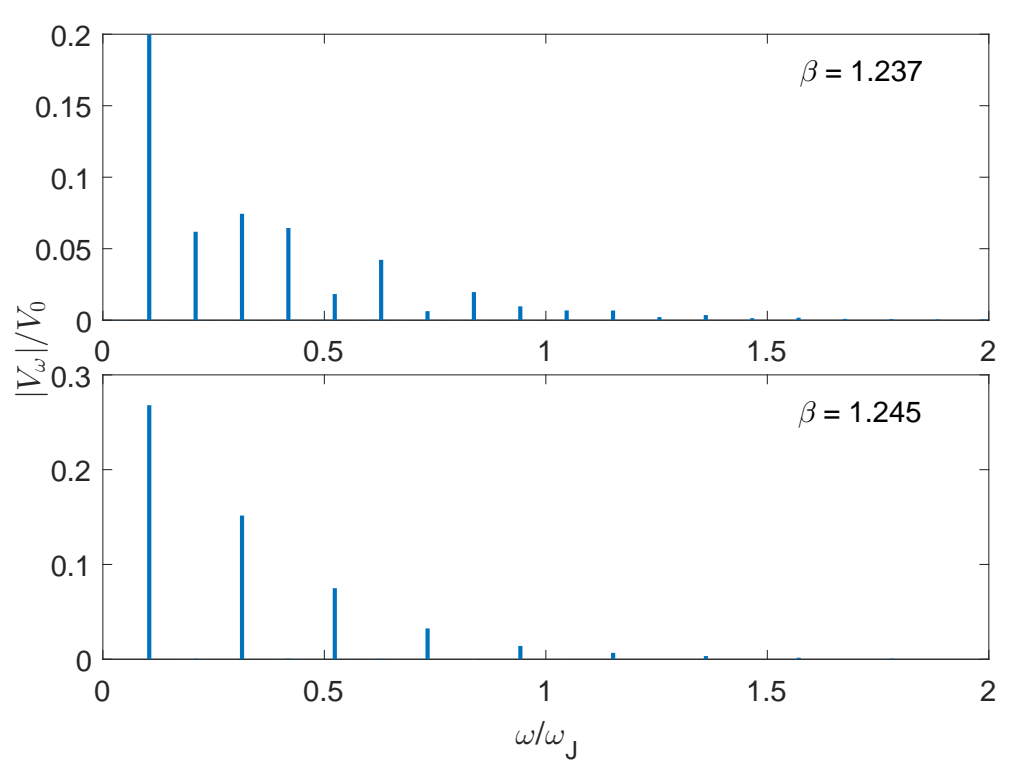

FIG. 68: Fourier spectrum of voltage at $\eta=2$ calculated for oscillatory vortex dynamics at $\beta_{0}=1.237$ and ballistic vortex penetration at $\beta_{0}=1.245$ represented in Figs. 66 and 67, respectively. The peaks in $V_{\omega}$ occur at the multiples of the ac frequency $\omega_{n}=n \omega$, where $\omega=\pi / 30$ and $n=1,2,3, \ldots$. Notice that voltage harmonics with even $n$ disappear as the vortex dynamics changes from oscillatory to ballistic.

models where vortices penetrate only through the edges of the junction (Fig. $71 \mathrm{~b}$ ), here new vortices will be produced inside the junction without penetrating through the edges. This mechanism will continue on until at $\beta=1.092$, the excessive radiation due to oscillation of vortices that was of the form of a decaying hump at smaller currents, becomes strong enough that produces a pair of v-av inside the junction, as displayed in Fig. 72. Here the mechanism of pair production is different in the sense that the instability is nucleated away from the moving vortex. This is where the second jump in the graph of dissipation occurs. Figure 72 shows the intial stage when this radiaiotn hump turns into a pair of v-av, but the periodic solution afterwards is shown in Fig. 73a. For currents up to $\beta_{0}=1.098$ the pair of vortices oscillate going throug each other while the edge vortex is replaced by antivortex in the following negative cycle. However, as $\beta_{0}$ increases further and exceeds 1.098, only one vortex and one antivortex remain in the junction so that the power $\bar{P}\left(\beta_{0}\right)$ drops. As shown in Fig.73b, in this regime in each current cycle one vortex and one antivortex penetrate the junction from both edges. Penetration depth of each fluxon increases as current enhances (Fig.73c) until at $\beta_{0}=1.149$ the pair of v-av gets so close above that the solution becomes a ballistic penetration of one voretx/antivortex at each cycle. At larger $\beta_{0}$ there is a complex 

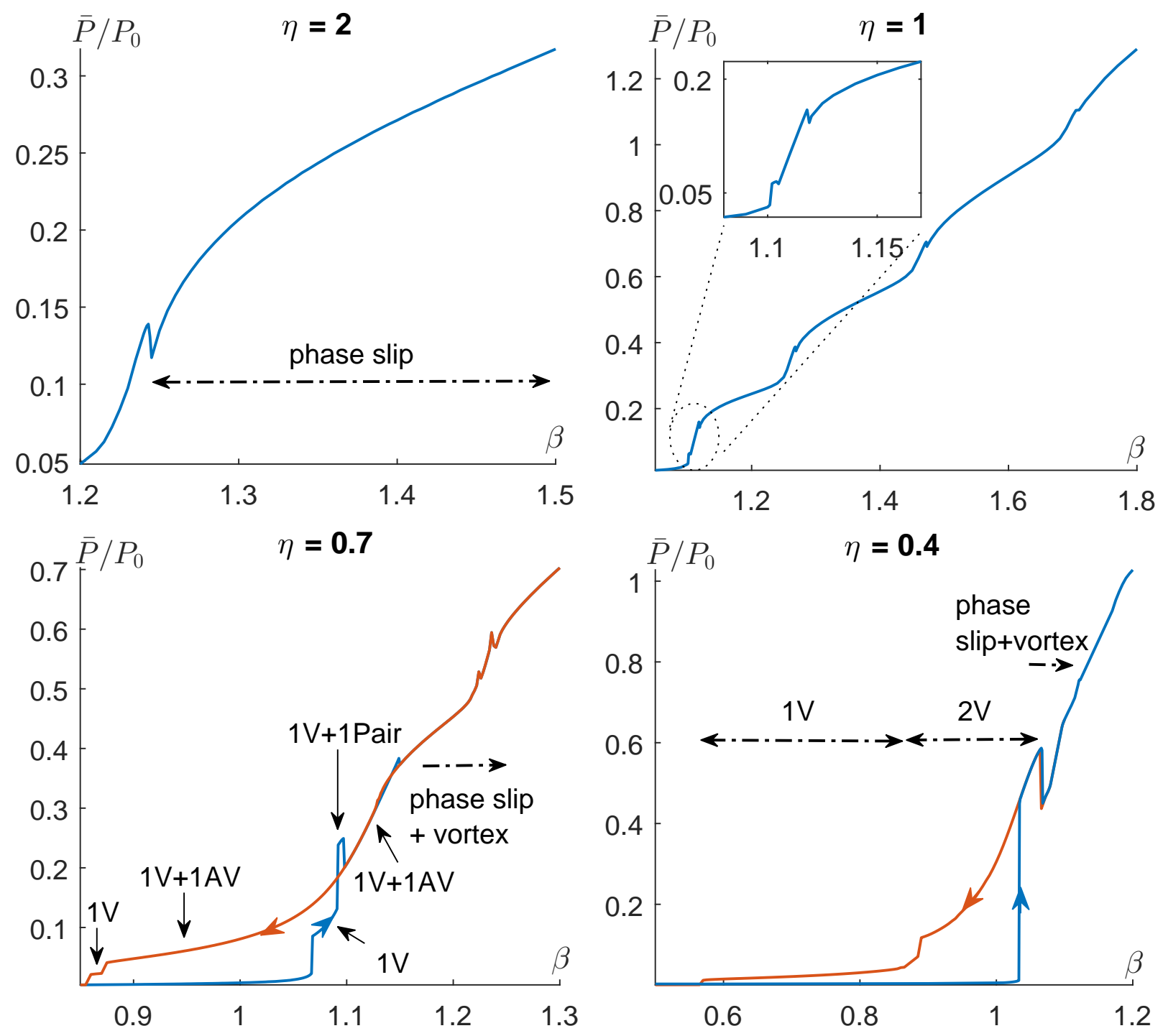

FIG. 69: AC power plots $P\left(\beta_{0}\right)$ for different damping constants. At large currents $\beta_{0} \gtrsim 3$, the curves $P\left(\beta_{0}\right)$ approach the ohmic limit $P=P_{0} \beta_{0}^{2} / 2 \eta$. 


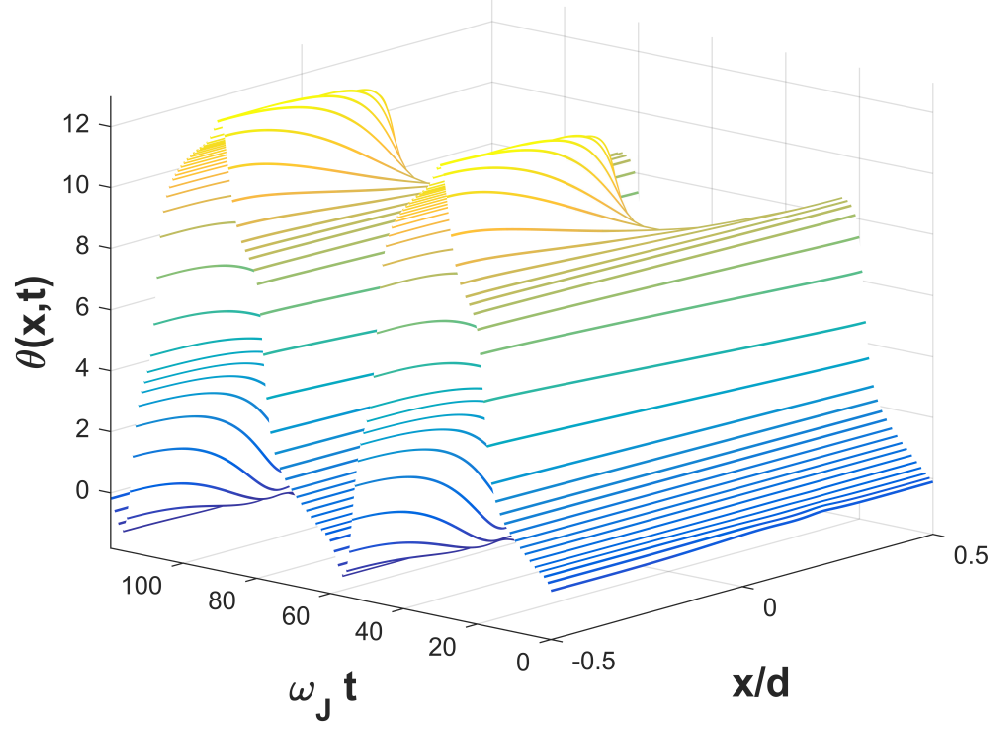

FIG. 70: Each large step in dissipation plots corresponds to an additional vortex penetration during ac current cycles. Shown above is the second vortex partial penetration above a ballistic motion of vortices calculated at $\eta=1$ and $\beta_{0}=1.26$.

superposition of ballistic and oscillating vortex states which eventually evolves into the phase slip state as $\beta_{0}$ increases [140]. On the descending branch of $\bar{P}\left(\beta_{0}\right)$ the phase slip state turns into an oscillating penetration of one vortex and one antivortex from the opposite edges at $\beta_{0}<1.132$ until the penetration stops at $\beta_{0}=0.875$.

At $\eta=0.4$ the first big jump on the ascending branch of $\bar{P}\left(\beta_{0}\right)$ shown in Fig. 69 occurs at $\beta_{0}=1.034$ as a pair of two radiating vortices penetrate the junction during the positive ac cycle, stop midway and return during the negative ac cycle (Fig. 74). Similar to the case of $\eta=0.7$, each of these two vortices produce a v-av pair, then all vortices exit and two antivortices remain. In turn, these antivortices repeat the same process during the positive ac cycle. As $\beta_{0}$ increases vortices penetrate deeper into the junction until the motion of the vortex pair becomes ballistic and $\bar{P}\left(\beta_{0}\right)$ drops at $\beta_{0}=1.068$. At higher currents signs of chaotic dynamics of oscillating vortices coexisting with ballistic vortices appear. In the region of currents $\beta_{0} \simeq 1.2-2$ simulation of Eq. (106) becomes very time consuming as it does not have an apparent time-periodic solution. Yet as ac current amplitude increases, the phase slip state eventually takes over for which $\bar{P}\left(\beta_{0}\right)$ follows that of a point junction and turns into a quadratic ohmic dependence at larger ac amplitudes. On the descending branch of $\bar{P}\left(\beta_{0}\right)$, two oscillating vortices remain in the junction all the way to $\beta_{0}=0.89$. At lower currents a step in $\bar{P}\left(\beta_{0}\right)$ at $\beta_{0}=0.8$ occurs as only one vortex remains in the junction 
during positive ac cycle followed by one antivortex during negative ac cycle, until neither of them can exist in the junction at $\beta_{0}<0.57$.

Our simulations of Eq. (106) at $\eta<0.3$ have shown that the vortex starts producing a cascade of v-av pairs right after it enters the junction. In this case the junction goes into a resistive state which exhibits a stochastic phase slip state coexisting with a random appearance of single vortices and antivortices even at high currents. For instance, Fig. 75, which plots the magnitude of $\theta( \pm d / 2, t)$ at any instant of time, illustrates the dominance of phase slip behavior of junction for most of the time with random appearance of a vortex at $t \simeq 320$. Apart from stochastic appearance of vortices, the phase slip perfectly resembles the behavior of a point junciton in ac current. Similar results were observed for the case of a point defect at the edge of the junction under ac current (more simulations can be found in Ref. [140]).

\subsection{SUMMARY AND DISCUSSION}

In this chapter we addressed nonlinear dynamics of vortices driven by strong dc and ac currents in long but finite Josephson junctions in which nonlocality of Josephson electrodynamics is essential. Behavior of AJ vortices in such junctions turns out to be rather different from either J or A vortices. Our numerical simulations and analytical results show that moving vortices in thin film junctions can exist only up to currents close to the critical current of the junction. As the current increases, driven vortex structures evolve into a dynamic phase slip state with a nearly flat phase profile $\theta(x, t)$ similar to that of a point junction. The vortex-to-phase slip transition caused by the Josephson nonlocality occurs even in junctions which are much longer than the size of a static vortex, but the mechanisms of this transition is markedly different in overdamped and underdamped junctions. In overdamped junctions the vortex-to-phase slip transition occurs because the length of the vortex increases strongly as current increases, so that the vortex solutions disappear as the length of the vortex becomes of the order of the length of the junction. This conclusion follows from the exact solution[137] for a driven AJ vortex at $\eta \gg 1$ and numerical simulations of (106).

In underdamped junctions the vortex-to-phase slip transition results from radiation of vortices which produce strong Cherenkov wakes and bremsstrahlung caused by interaction of vortices with the junction edges and other vortices. These effects trigger generation of $\mathrm{v}$-av pairs inside the junction which become more pronounced as the damping constant $\eta$ decreases. At $\eta<0.3$ our simulations show that the vortex penetrating from the edge of the junction produces a cascade of expanding v-av pairs driving the entire junction into the the 

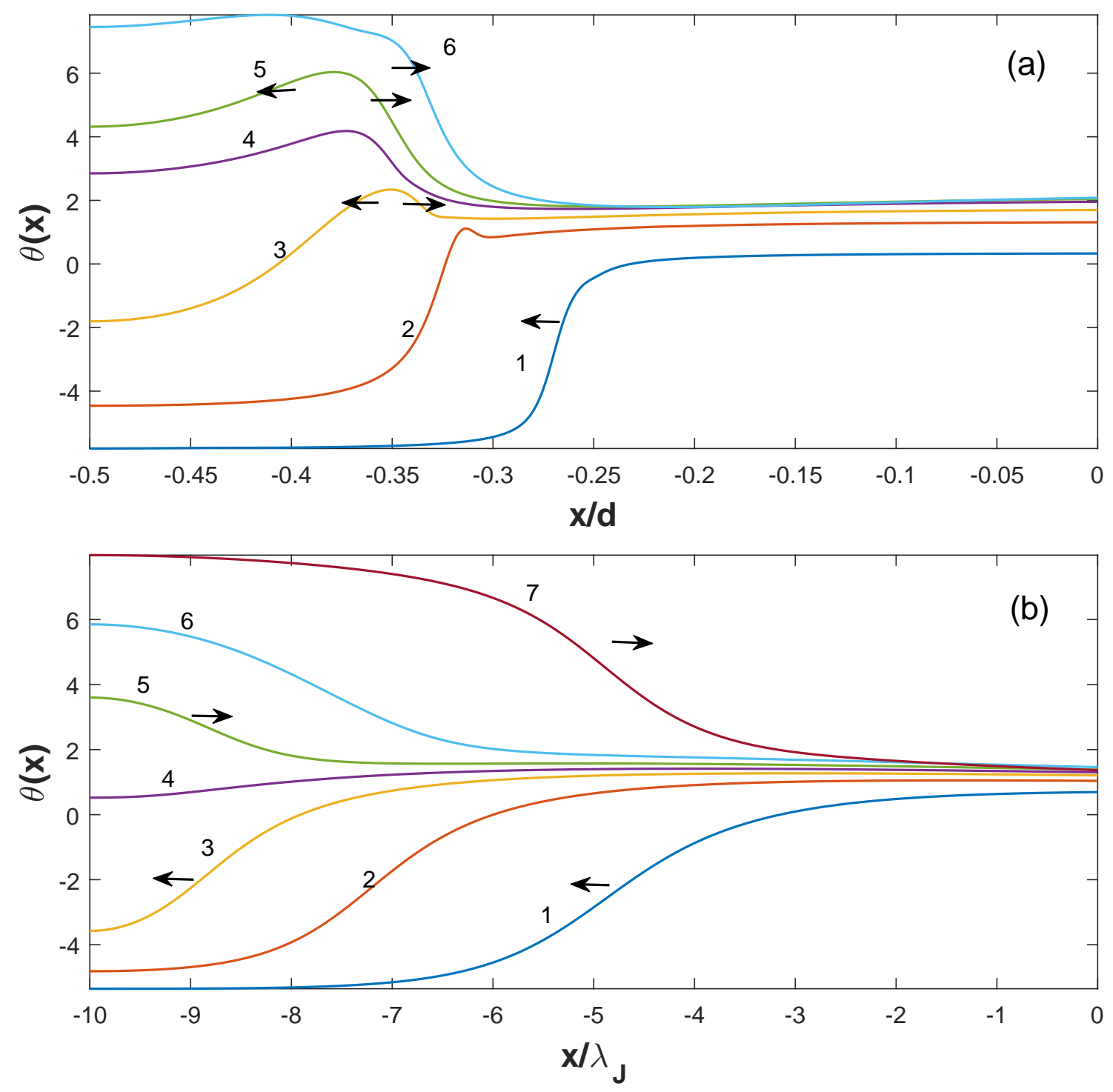

FIG. 71: Mechanism of vortex penetration into the junction calculated at $\eta=0.7$ for (a) nonlocal model at $\beta_{0}=1.085$ and (b) local at $\beta_{0}=1.01$. Vortices are produced through pair production inside the underdamped junction in thin film nonlocal model. 


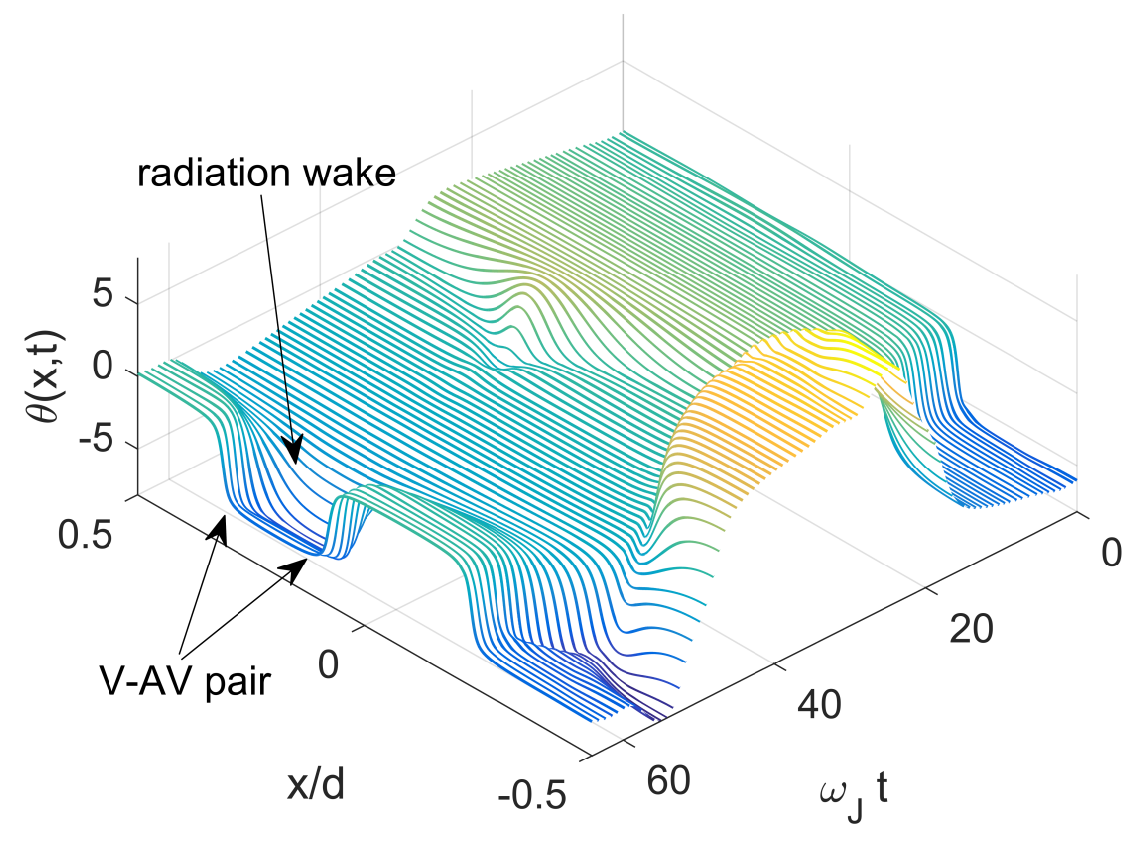

FIG. 72: Generation of a v-av pair due to radiation of a penetrating vortex at $\eta=0.7$ at $\beta_{0}=1.092$. The dynamics becomes periodic afterwards which is shown in Fig.73a.

phase slip state. In this case the $V-I$ curves become hysteretic, vortices emerge from the phase slip state as the current is decreased on the return branch of $V(I)$. Under ac currents dynamics of vortices appear stochastic at small $\eta$ and intermediate currents $\beta_{0} \sim 1$, while the phase slip behavior is still dominant at ac amplitudes $\beta_{0} \gg 1$.

Our calculations of $V-I$ characteristics and the power $P\left(\beta_{0}\right)$ dissipated by moving vortices show that $V\left(\beta_{0}\right)$ and $P\left(\beta_{0}\right)$ can be complicated functions of the amplitude $\beta_{0}$ of dc or ac current, and have regions with negative differential resistance $d V / d I$ and jump-wise hysteretic transitions. This situation is particularly relevant to underdamped junctions and grain boundaries at low temperatures in such materials like $\mathrm{Nb}_{3} \mathrm{Sn}$, iron-based superconductors and cuprates in which grain boundaries behave as planar weak links $[2,11]$. In this case vortices moving along networks of grain boundaries of these policrystalline materials can significantly contribute to the flux flow resistance and power dissipated under dc or ac currents, resulting in new mechanisms of nonlinearity of electromagnetic response associated with the dynamics of AJ vortices. These effects are essential for the understanding of the nonlinear residual surface resistance in polycrystalline resonator cavities and thin film multilayer screens under strong RF electromagnetic field.

Proliferation of v-av pairs caused by moving vortices can be essential for the physics and 

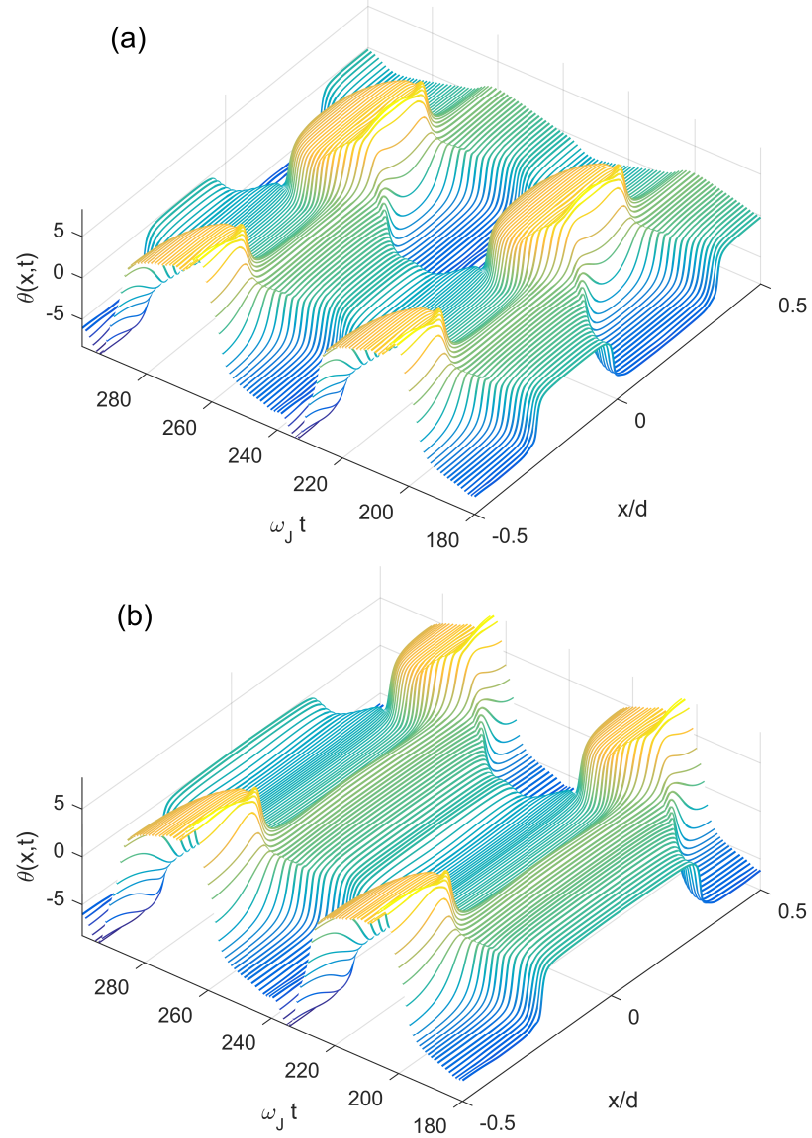

(c)

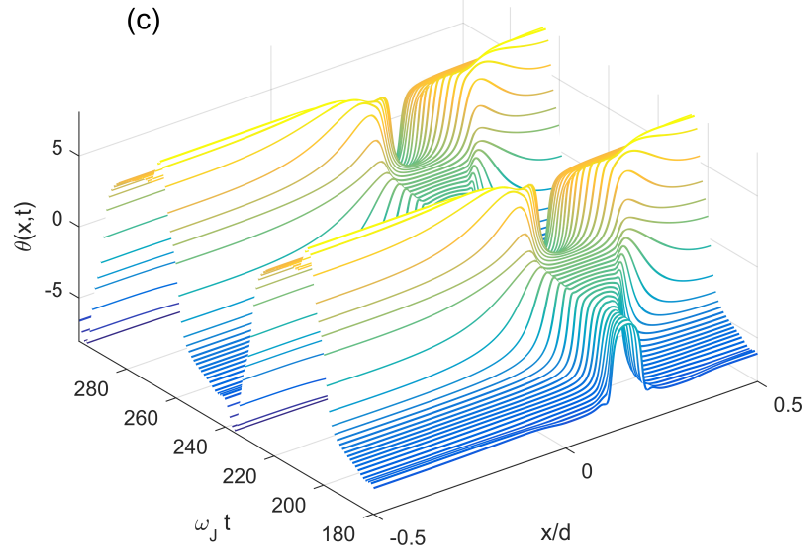

FIG. 73: (a) Generation of a pair as due to excessive radiation produces the spike in Fig.69 for $\eta=0.7$ and $\beta_{0}=1.092$. With increase of current the amplitude of oscillations of pair increases until at (b) $\beta_{0}=1.098$ the vortex of the pair annihilates with left penetrating antivortex and the junction stabilizes after this transient with one vortex and one antivortex penetrating from edges. This drop in number of vortices also causes the power to drop. (c) Further increase of current causes the vortex and antivortex to become so close such that the solution turns into a ballistic penetration of vortex/antivortex at each current cycle. 


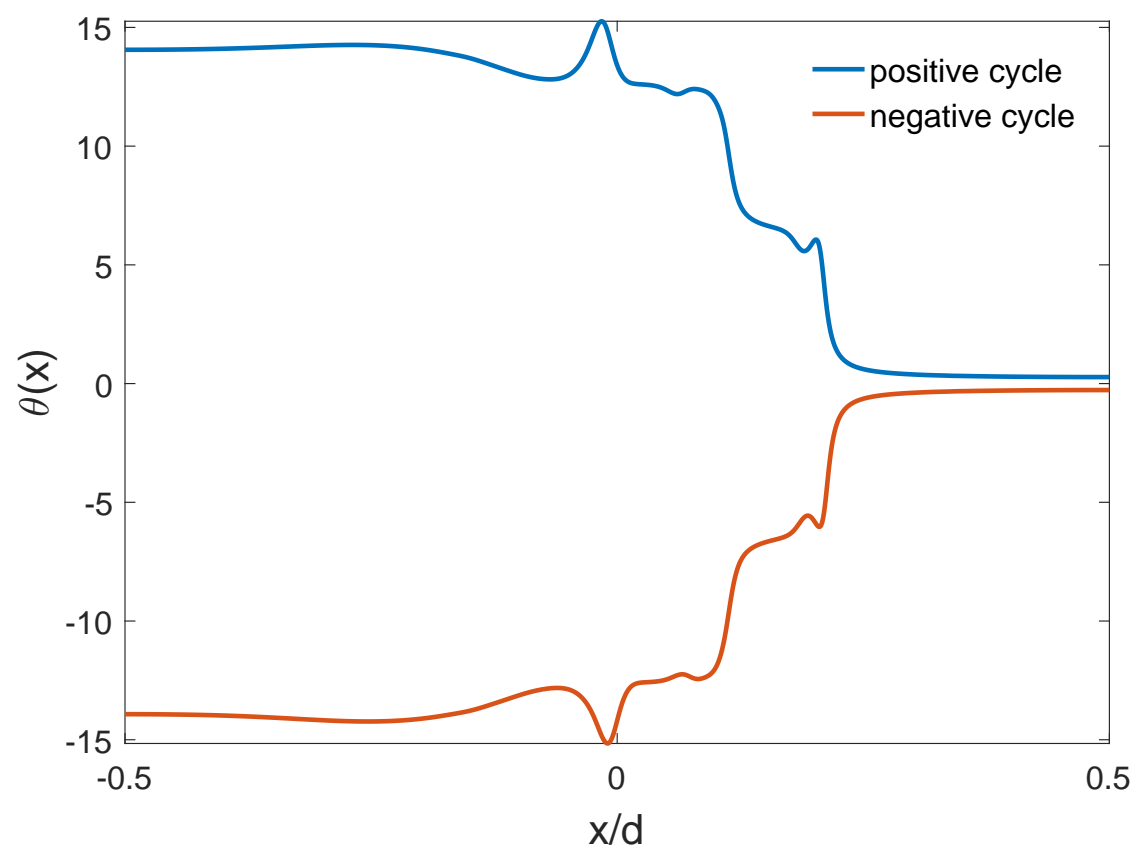

FIG. 74: Penetration of radiating vortices and antivortices at $\eta=0.4$ and $\beta_{0}=1.034$ during positive and negative current cycles.

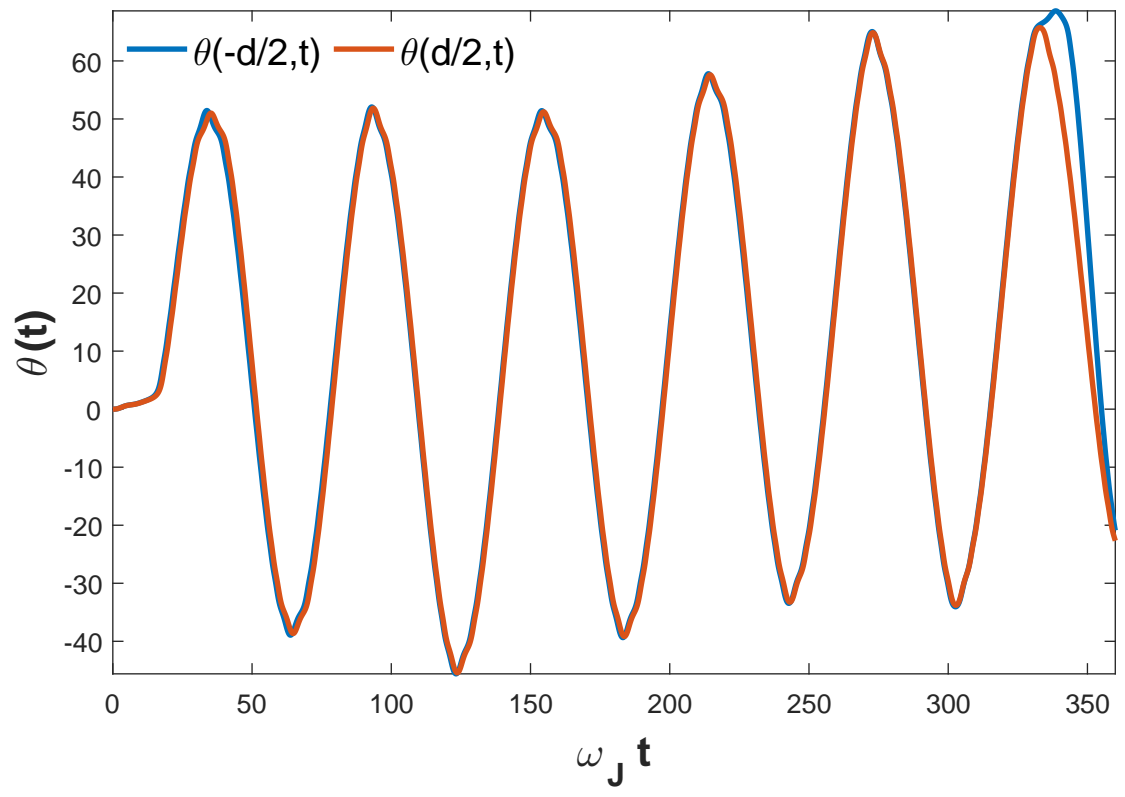

FIG. 75: Dynamics of $\theta(-d / 2, t)$ and $\theta(d / 2, t)$ at the edges at $\eta=0.2$ and $\beta_{0}=1.1$. Here $\theta(x, t)$ remains nearly uniform along junction, indicating a phase slip behavior. 
applications of weak link superconducting structures in which the dynamic vortex instabilities can result in hysteretic jumps on the $V-I$ curves which appear similar to those produced by overheating [43]. However, the effects discussed here are affected by neither cooling conditions nor the nonequilibrium kinetics of quasiparticles. Heating is most pronounced in overdamped junctions in which radiation is suppressed, while the generation of v-av pairs is characteristic of underdamped junctions. Yet the big jumps of the $V-I$ curves caused by penetration of vortex bundles into underdamped junctions can result in significant local heating which, in turn, can trigger thermal instabilities.

As noted before, the effects addressed here do not require special junctions with $J_{c} \sim J_{d}$ : the Cherenkov instability caused by weak nonlocal Josephson electrodynamics effects occurs even in a planar weak link with $\lambda_{J}=10 \lambda$ as shown in Fig. 39, whereas in thin film edge junctions the nonlocality becomes essential at much lower $J_{c}$. The result of this work may also pertain to the transition of A vortices driven by strong currents into chains of weakly coupled $\mathrm{J}$ vortices or phase slips in wide thin films $[141,142,143,144,145,146,147]$. In this case vortices moving along a self-induced channel of reduced order parameter behave as overdamped AJ vortices considered here. As the current increases the AJ vortices further elongate along the flux channel and move faster, so we may expect a transition from the AJ vortices to a phase slip state above a threshold current in a film strip, similar to that for a Josephson junction of finite length. 


\section{CHAPTER 6}

\section{CONCLUSIONS}

This work provides the results of an extensive numerical simulation of the nonlinear dynamics of Josephson and mixed Abrikosov-Josephson vortices along with a detailed understanding of the mechanisms of power dissipation in both weak-link and strong-link grain boundaries in bulk and thin superconducting polycrystalline film screens under strong dc and ac electromagnetic fields. In particular, we showed that the electromagnetic response of a weak-link (low- $J_{c}$ ) grain boundary in polycrystalline materials like $\mathrm{Nb}_{3} \mathrm{Sn}$, iron-based or high- $T_{c}$ superconductors can be quite complicated due to penetration, oscillation and annihilation of Josephson vortices. These nonlinear dynamic effects result in a nonlinear dependence of the average dissipated power and the dynamic surface resistance which can have steps and peaks corresponding to the different number of trapped vortices in the junction as the field increases. It has been shown that at fields larger than the threshold of vortex penetration the behavior of power becomes quadratic while the surface resistance saturates to values of the order of normal resistance of junction. Numerical values have been estimated for power spikes due to annihilation of vortices and antivortices inside the junction. In ac field applications, the threshold amplitude for penetration of vortices $H_{p}(\omega)$ was shown to be a linear function of frequency with slope $\propto 1 / \omega_{c}$. The characteristic frequency $\omega_{c}$ is proportional to $J_{c} R_{j}$, so the frequency dependence is most pronounced in low- $J_{c}$, low resistance grain boundaries and $H_{p}(\omega)$ remains close to that of dc Josephson superheating field $H_{1}$ if $\omega \ll \omega_{c}$. Therefore, one can expect that in SRF materials like $\mathrm{Nb}_{3} \mathrm{Sn}$ and multilayer structures, the nonlinear effects addressed here can manifest themselves in surface impedance at rather low fields $H \simeq H_{1} \sim H_{c}\left(J_{c} / \kappa J_{d}\right)^{1 / 2}$ which can be much less than the bulk superheating field at which the Meissner state becomes unstable.

Grain boundaries in $\mathrm{Nb}$ resonator cavities appear to be strongly-coupled due to the fact that some of the best performing $\mathrm{Nb}$ cavities are fabricated from small grain polycrystalline niobium sheets. Therefore, vortices do not penetrate up to very high magnetic fields at which the screening current densities are of the order of depairing current density. Moreover, the dynamic behavior of these strongly-coupled GBs cannot be described by conventional models of Josephson junctions which are based on a local relation between field and phase. Taking into account the nonlocal effects, a more general integro-differential equation needs 
to be solved numerically which reveals the dynamics of mixed Abrikosov-Josephson vortices. Our simulation results of this integral equation predicts that a uniformly moving vortex in an underdamped junction which radiates Cherenkov waves can become unstable at some critical current which can be well below $J_{c}$. The instability occurs in the form of continuous generation of vortex-antivortex pairs which eventually evolves as a phase-pile in the middle of an infinitely long junction. The instability effects become even more essential once the effects of the finite size of a GB is considered. The results of simulation for a finite size GB in a thin film screen under dc and ac magnetic fields show that moving vortices in thin film GBs can exist only up to currents close to the critical current of the GB. As current increase moving vortices evolve into dynamic phase slips which turn the whole GB into resistive state. However, the mechanisms of this transition markedly changes with properties of GBs or temperature. At low temperatures this transition to resistive state is a result of collective effects of two different radiation sources, namely, Cherenkov radiation and bremsstrahlung caused by interaction of vortices with junction edges and other vortices. In this case the $V-I$ characteristics and the dissipated power curves $P(J)$ can be complicated functions of $\mathrm{dc}$ and ac current amplitude and have regions with negative differential resistance and jump-wise hysteretic transitions, although power generally becomes a quadratic function of current amplitude at $J \gtrsim 3 J_{c}$.

In superconducting materials with large G-L parameter $\kappa$, the nonlocal effects such as instability can happen in a wide range of currents, $J_{d} / \kappa<J<J_{d}$. Moreover, simulations showed that the nonlocal effects can be essential even in a planar weak-link GB with $\lambda_{J}=10 \lambda$ $\left(J_{c} \sim 10^{-2} J_{d} / \kappa\right)$ where the instability happens at $\sim 0.9 J_{c}$. However, the prominence of the nonlocal effects in underdamped junctions (low temperatures) implies that instability is neither affected by cooling conditions nor by kinematics of quasiparticles as both of these issues are most pronounced in overdamped junctions in which radiation is suppressed.

While we believe this work provides an unprecedented detailed understanding of the physics of penetration and dissipation of vortices in GBs, it still can greatly benefit from investigations which specifically address the microstructure of GBs in SRF materials. Although rough numerical estimations have been provided when possible, the lack of information about the characteristic properties of GBs in such materials of interest, limits our ability to make precise predictions about the exact contribution of GBs to residual resistivity of any specific material. The results of this work will be applicable to wide variety of other systems which share similar dynamics of topological defects as vortices. 


\section{BIBLIOGRAPHY}

[1] X. Y. Song, G. Daniels, D. M. Feldmann, A. Gurevich, and D. Larbalestier, Nature Mater. 4, 470 (2005).

[2] H. Hilgenkamp and J. Mannhart, Rev. Mod. Phys. 74, 485 (2002).

[3] A.-M. Valente-Feliciano, Supercon. Sci. Technol. 29, 113002 (2016).

[4] S. Posen and D. Hall, Superdon. Sci. Technol. 30, 033004 (2017).

[5] M. Tinkham, Introduction to Superconductivity (New York, McGraw-Hill, 2nd ed., 1996).

[6] H. Padamsee, J. Knobloch, and T. Hays, RF Superconductivity for Accelerators (New York, Wiley, 2nd ed., 2007).

[7] C. Z. Antoine, Materials and surface aspects in the development of SRF niobium cavities (EUCARD-BOO-2012-001).

[8] A. Gurevich, Rev. Accel. Sci. Technol. 5, 119-146 (2012).

[9] H. Padamsee, Annu. Rev. Nucl. Part. Sci. 64, 175 (2014).

[10] A. Gurevich, Supercond. Sci. Technol. 30, 034004 (2017).

[11] J. H. Durrell, C. B. Eom, A. Gurevich, E. E. Hellstrom, C. Tarantini, A. Yamamoto, and D. C. Larbalestier, Rep. Prog. Phys 74, 124511 (2011).

[12] A. M. Campbell and J. E. Evetts, Adv. Phys. 21, 199 (1972).

[13] A. Gurevich, Appl. Phys. Lett. 88, 012511 (2006).

[14] A. Gurevich, Presented at SRF 12th Workshop on RF Superconductivity, Cornell University, Ithaca, NY July 10-15, 2005.

[15] B. Bonin and H. Safa, Supercond. Sci. Technol. 4, 257 (1991).

[16] C. Attanasio, L. Maritato, and R. Vaglio, Phys. Rev. B 43, 6128 (1991).

[17] J. Halbritter, J. Appl. Phys. 97, 083904 (2005). 
[18] C. Benvenuti, S. Calatroni, I. E. Campisi, P. Darriulat, M. A. Peck, R. Russo, and A.-M. Valente, Physica C 316, 153 (1999).

[19] C. Benvenuti, S. Calatroni, P. Darriulat, M. A. Peck, A.-M. Valente, and C. A. V. Hof, Physica C 351, 421 (2001).

[20] S. Calatroni, Physica C 441, 95 (2006).

[21] R. L. Geng, Physica C 441, 145 (2006).

[22] R. L. Geng, G. V. Eremeev, H. Padamsee, and V. D. Shemelin, Proc. PAC07 (Albuquerque, New Mexico, USA), 2337 (2007).

[23] Z. H. Sung, The Influence of Grain Boundaries on the Properties of Superconducting Radio Frequency Cavity Niobium (Doctoral thesis), 2006.

[24] J. Knobloch, R. L. Geng, M. Liepey, H. Padamsee, and F. R. Newman, 8th SRF workshop, Snata Fe, USA, 1990.

[25] J. Halbritter, J. Supercond. 10, 91 (1997).

[26] G. Ciovati and J. Halbritter, Physica C 441, 57 (2006).

[27] F. Silsbee, J. Wash. Acad. Sci. 6, 597 (1916).

[28] F. London and H. London, Proc. Roy. Soc. A 141, 547 (1935).

[29] J. Bardeen, L. N. Cooper, and J. R. Schrieffer, Phys. Rev. 108, 1175 (1957).

[30] V. L. Ginzburg and L. D. Landau, Zh. Eksperim. i. Teor. Fiz. 20, 1064 (1950).

[31] A. A. Abrikosov, Zh. Eksperim. i. Teor. Fiz. 32, 1442 (1957).

[32] G. Bednorz and K. A. Müller, Z. Phys. B 64, 189 (1986).

[33] D. C. Mattis and J. Bardeen, Phys. Rev. 111, 412 (1958).

[34] J. P. Turneaure and I. Weissman, J. Appl. Phys. 39, 4417 (1968).

[35] A. Andreone, A. Cassainese, M. Iavarone, R. Vaglio, I. I. Kulik, and V. Palmieri, Phys. Rev. B 52, 4473 (1995). 
[36] T. Proslier, J. F. Zasadzinskii, J. Moore, L. Cooley, C. Antoine, M. Pellin, J. Norem, and K. Gary, Appl. Phys. Lett. 92, 212505 (2008).

[37] C. B. et al, Appl. Phys. Lett. 106, 082602 (2015).

[38] J. McDonald and J. R. Clem, Phys. Rev. B 56, 14723 (1997).

[39] Z. Zhai, P. V. Parimi, and S. Sridhar, Phys. Rev. B 59, 9573 (1999).

[40] B. D. Josephson, Phys. Lett. 1, 251 (1962).

[41] B. D. Josephson, Adv. Phys. 14, 419 (1965).

[42] A. Barone and G. Paterno, Physics and Applications of Josephson Effects (New York: Wiley, 1982).

[43] K. K. Likharev, Dynamics of Josephson Junctions and Circuits (New York, Gordon and Breach Science Publishers, 1986).

[44] P. W. Anderson and J. M. Rowell, Phys. Rev. Lett. 10, 230 (1963).

[45] R. P. Feynman, the Feynman Lectures on Physics: Vol III.

[46] V. Ambegaokar and A. Baratoff, Phys. Rev. Lett. 10, 486 (1963).

[47] I. K. Yanson, V. M. Svitsunov, and I. M. Dmitrenko, Sov. Phys. -JETP 21, 650-652 (1965).

[48] I. Giaever, Phys. Rev. Lett. 14, 904 (1965).

[49] K. K. Likharev, Rev. Mod. Phys. 51, 101 (1979).

[50] W. C. Stewart, Appl. Phys. Lett. 12, 277 (1968).

[51] D. E. McCumber, J. Appl. Phys. 39, 3113 (1968).

[52] S. Shapiro, Phys. Rev. Lett. 11, 80 (1963).

[53] G. Gruner, Rev. Mod. Phys. 60, 1129-1181 (1988).

[54] V. L. Pokorovsky and A. L. Talapov, Phys. Rev. Lett. 42, 65-67 (1979).

[55] P. Bak, Rep. Prog. Phys. 45, 587-629 (1982). 
[56] V. G. Bar'yakhtar, M. V. Chetkin, B. A. Ivanov, and S. N. Gadetskii, Dynamics of Topological Magnetic Solitons (Berlin, Heidelberg, New York: Springer-Verlag, 1994).

[57] J. B. Hirth and J. Lothe, Theory of Dislocations (New York: McGraw-Hill, 1968).

[58] O. M. Braun and Y. S. Kivshar, The Frenkel-Kontorova Model: Concepts, Methods and Applications (Berlin, New York, Springer-Verlag, 2004).

[59] M. Salerno, Phys. Rev. A 44, 5292-5297 (1991).

[60] L. V. Yakushevich, Nonlinear Physics of DNA (Weinheim, John Wiley and Sons, 2nd ed., 2006).

[61] J. C. Swihart, J. Appl. Phys. 32, 461 (1961).

[62] D. W. McLaughlin and A. C. Scott, Phys. Rev. A 18, 1652 (1978).

[63] N. Gronbech-Jensen, Phys. Rev. B 45, 7315 (1992).

[64] T. A. Fulton and R. C. Dynes, Solid State Comm., 12, 57 (1973).

[65] S. N. Erne, A. Ferrigno, and R. D. Parmentier, Phys. Rev. B 27, 5440 (1983).

[66] R. D. Parmentier, arXiv:patt-sol/9303003v1, 1993.

[67] T. Nagatsuma, K. Enpuku, K. Yoshida, and F. Irie, J. Appl. Phys. 56, 3284 (1984).

[68] P. Lebwohl and M. J. Stephen, Phys. Rev. 163, 376 (1967).

[69] M. Cirillo, M. Gronbech-Jensen, M. R. Samuelsen, M. Salerno, and G. V. Rinati, Phys. Rev. B 58, 12377 (1988).

[70] V. P. Koshelets and S. V. Shitov, Supercond. Sci. Techno. 13, R53 (2000).

[71] M. Salerno and M. R. Samuelsen, phys. Rev. B 59, 14653 (1999).

[72] M. Salerno and M. Samuelsen, Nonlinear Science at the Dawn of the 21st Century, 542, LNP (2000).

[73] T. M. Hylton and M. R. Beasley, Phys. Rev. B 39, 9042 (1989).

[74] A. Gurevich, Annu. Rev. Cond. Matt. Phys. 5, 35 (2014). 
[75] H. Safa, M. Bolor, Y. Boudigou, S. Jaidane, R. Keller, P. Nardin, and G. Szegedi, Proc. 1999 Workshop on RF Superconductivity, 029, (Santa Fe, New Mexico, USA), 267, TUP.

[76] A. Gurevich, Phys. Rev. B 46, R3187 (1992).

[77] A. Gurevich, Physica C 243, 191 (1995).

[78] A. Gurevich, Phys. Rev. B 65, 214531 (2002).

[79] Y. M. Habib, C. J. Lehner, D. E. Oates, L. R. Vale, R. H. Ono, G. Dresselhaus, and M. S. Dresselhaus, Phys. Rev. B 57, 13833 (1998).

[80] J. Nagel, D. Speer, T. Gaber, A. Sterck, R. Eichhorn, P. Reimann, K. Ilin, M. Seigel, D. Koelle, and R. Kleiner, Phys. Rev. Lett 100, 217001 (2008).

[81] M. Salerno and Y. Zolotaryuk, Phys. Rev. E 65, 056603 (2002).

[82] A. V. Ustinov, C. Coqui, A. Kemp, Y. Zolotaryuk, and M. Salerno, Phys. Rev. Lett. 93, 087001 (2004).

[83] E. Zamora-Sillero, N. R. Quintero, and F. G. Mertens, Phys. Rev. E 74, 046607 (2006).

[84] H. Linke Appl. Phys. A: Mater. Sci. Process 75, 167 (2002).

[85] P. Hänggi and F. Marchesoni Rev. Mod. Phys. 81, 387 (2009).

[86] S. Matthias and F. Muller, Nature 424, 53 (2003).

[87] J. E. Villegas, S. Savel'ev, F. Nori, E. M. Gonzalez, J. V. Anguita, R. Garcia, and J. L. Vicent, Science 302, 1188 (2003).

[88] S. Savel'ev and F. Nori, Nature Mater. 1, 179 (2002).

[89] D. Cole, S. Bending, S. Savel'ev, A. Grigorenko, T. Tamegai, and F. Nori, Nature Mater. 5, 305 (2006).

[90] M. Beck, E. Goldobin, M. Neuhaus, M. Siegel, R. Kleiner, and D. Koelle, Phys. Rev. Lett. 95, 090603 (2005).

[91] A. Gurevich and G. Ciovati, Phys. Rev. B 77, 104501 (2008). 
[92] J. Pearl, Appl. Phys. Lett. 5, 65 (1964).

[93] Y. M. Ivanchenko and T. K. Soboleva, Phys. Lett. A 147, 65-69 (1990).

[94] A. Gurevich, Phys. Rev. B 48, 12857 (1993).

[95] R. G. Mints and I. B. Snapiro, Phys. Rev. B 51, 3054 (1995).

[96] R. G. Mints and I. B. Snapiro, Phys. Rev. B 52, 9691 (1995).

[97] Y. E. Kuzovlev and A. I. Lomtev, Sov. Phys. -JETP 84, 986 (1997).

[98] V. P. Silin, Sov. Phys. -JETP 85, 760 (1997).

[99] V. G. Kogan, Phys. Rev. B. 49, 15874 (1994).

[100] V. G. Kogan, V. V. Doborovski, J. R. Clem, Y. Mavatari, and R. G. Mints, Phys. Rev. B 63, 144501 (2001).

[101] Y. M. Aliev, V. P. Silin, and S. A. Uryupin, Supercond. Phys. Chem. Technol. 5, 230 (1992).

[102] G. L. Alfimov and A. F. Popkov, Phys. Rev. B 52, 4503 (1995).

[103] G. L. Alfimov and A. F. Popkov, Phys. Rev. B 73, 214512 (2006).

[104] A. A. Abdulmalikov, G. L. Alfimov, and A. S. Malishevskii, Supercond. Sci. Technol. 22, 023001 (2009).

[105] A. Gurevich, M. S. Rzchowski, G. Daniels, S. Patnaik, B. M. Hinaus, F. Carillo, F. Tafuri, and D. C. Larbalestier, Phys. Rev. Lett. 88, 097001 (2002).

[106] T. Horide, K. Matsumoto, A. Ichinose, M. Mukaida, Y. Yoshida, and S. Horii, Phys. Rev. B 75, 020504(R) (2007).

[107] T. Horide, K. Matsumoto, Y. Yoshida, M. Mukaida, A. Ichinose, and S. Horii, Phys. Rev. B 77, 132502 (2008).

[108] A. V. Ustinov, T. Doderer, R. P. Huebener, N. F. Pedersen, B. Mayer, and V. A. Oboznov, Phys. Rev. Lett. 69, 1815 (1992).

[109] E. Goldobin, B. A. Malomed, and A. V. Ustinov, Phys. Rev. B 62, 1414 (2000). 
[110] J. Zitzmann, A. V. Ustinov, M. Levitchev, and S. Sakai, Phys. Rev. B 66, 064527 (2002).

[111] A. Abdulmalikov, M. Fitsul, and A. Ustinov, Phys. Rev. B 72, 144526 (2005).

[112] A. A. Boris, A. Rydh, T. Golod, H. Motzkau, A. M. Klushin, and V. Krasnov, Phys. Rev. Lett. 111, 117002 (2013).

[113] C. Brun, T. Cren, V. Cherkez, F. Debontridder, S. Pons, D. Fokin, M. C. Tringides, S. Bozhko, L. B. Ioffe, B. L. Altshuler, and D. Roditchev, Nature Phys. 10, 444-450 (2014).

[114] S. Yoshizawa, H. Kim, T. Kawakami, Y. Nagai, T. Nakayama, X. Hu, Y. Hasegawa, and T. Uchihashi, Phys. Rev. Lett. 113, 247004 (2014).

[115] D. Roditchev, C. Brun, L. Serrier-Garcia, J. C. Ceuvas, V. H. L. Bessa, M. V. Milośević, F. D. Debontridder, V. Stolyarov, and T. Cren, Nature Phys. 11, 332 (2015).

[116] A. Sheikhzada and A. Gurevich, Sci. Rep. 5, 17821 (2015).

[117] T. P. Orlando, W. J. McNiff, S. Foner, and M. R. Beasley, Phys. Rev. B. 19, 4545 (1979).

[118] R. Schinzinger and P. A. A. Laura, Conformal Mapping. Methods and Applications, (New York, Dover Publ, 2003).

[119] T. C. Edwards, Foundations for Microstrip Circuit Design, (Chichester, New York, Brisbone, Toronto, Wiley, 1981).

[120] M. P. Marder, Condensed Matter Phsyics (New Jersey, Wiley, 2010).

[121] A. Gurevich and R. G. Mints, Rev. Mod. Phys. 59, 941-999 (1987).

[122] U. Welp, K. Kadowaki, and R. Kleiner, Nature Photonics 7, 702-710 (2013).

[123] A. Gurevich and L. D. Cooley, Phys. Rev. B 50, 13563 (1994).

[124] G. R. Berdiyorov, M. V. Milosevic, S. Savelev, F. Kusmartsev, and F. M. Peeters, Phys. Rev. B 90, 134505 (2014).

[125] N. W. Ashcroft and N. D. Mermin, Solid State Physics (Philadelphia, Saunders College, 1976). 
[126] P. M. Chaikin and T. C. Lubensky, Principles of Condensed Matter Physics (Cambridge, New York, Madrid, Cambridge University Press, 2010).

[127] A. Herr, A. Fedorov, A. Shnirman, E. Il'ichev, and G. Schon, Supercond. Sci. Technol. 20, S450 (2007).

[128] M. Devoret and R. Schoelkopf, Science 339, 1169-1174 (2013).

[129] K. G. Fedorov, A. V. Shcherbakova, M. J. Wolf, D. Beckman, and A. Ustinov, Phys. Rev. Lett. 112, 160502 (2014).

[130] P. Cizeau, S. Zapperi, G. Durin, and H. E. Stanley, Phys. Rev. Lett. 79, 4669-4672 (1997).

[131] P. Rosakis, Phys. Rev. Lett. 86, 95-98 (2001).

[132] L. Pillon, C. Denoual, and Y. P. Pellegrini, Phys. Rev. B 76, 224105 (2007).

[133] A. Sheikhzada and A. Gurevich, Physica C 506, 59 (2014).

[134] G. Stejic, A. Gurevich, E. Kadyrov, D. Christen, R. Joynt, and D. C. Larbalestier, Phys. Rev. B 49, 1274 (1994).

[135] I. K. Yanson, V. M. Svistunov, and I. M. Dmitrenko, Zh. Eksp. Teor. Fiz. 48, 976 (1965). [Sov. Phys. -JETP 21, 650 (1965)]

[136] D. N. Langenberg, D. J. Scalapino, B. N. Taylor, and R. E. Eck, Phys. Rev. Lett. 15, 294 (1965).

[137] A. Sheikhzada and A. Gurevich, arxiv.org/abs/1703.02843, 2017.

[138] T. Golod, A. Rydh, and V. M. Krasnov, Phys. Rev. Lett. 104, 227003 (2010).

[139] V. G. Kogan and R. G. Mints, Phys. Rev. B 89, 014516 (2014).

[140] See [https://fs.wp.odu.edu/asheikhz/] for supplemental materials as well as simulation videos.

[141] A. Andronov, I. Gordion, V. Kurin, I. Nefedov, and I. Shereshevsky, Physica C 213, 193 (1993). 
[142] A. G. Sivakov, A. M. Glukhov, A. N. Omelyanchouk, Y. Koval, P. Müller, and A. V. Ustinov, Phys. Rev. Lett. 91, 267001 (2003).

[143] D. Y. Vodolazov and F. M. Peeters, Phys. Rev. B 76, 014521 (2007).

[144] G. R. Berdiyorov, M. Milośević, and F. Peeters, Phys. Rev. B 79, 184506 (2009).

[145] A. V. Silhanek, M. V. Milośević, G. R. B. R. B. G. Kramer, J. V. de Vondel, R. F. Luccas, T. Puig, F. M. Peeters, and V. V. Moshchalkov, Phys. Rev. Lett. 104, 017001 (2010).

[146] G. Berdiyorov, K. Harrabi, F. Oktasendra, K. Gasmi, A. I. Mansour, J. P. Maneval, and F. M. Peeters, Phys. Rev. B 90, 054506 (2014).

[147] G. Carapella, P. Sabatino, C. Barone, S. Pagano, and M. Gombos, Sci. Rep. 6, 35694 (2016).

[148] W. E. Schiesser, The Numerical Method of Lines: Integration of Partial Differential Equations (San Diego, Academic Press, 1991).

[149] L. F. Shampine and M. K. Gordon, Computer Solution of Ordinary Differential Equations: The Initial Value Problem (San Francisco, W. H. Freeman, 1975). 


\section{APPENDIX A}

\section{DERIVATION OF NONLOCAL FINITE JUNCTION MAIN EQUATION}

Equation (105) gives $g(x, 0)$ on the junction:

$$
\begin{gathered}
g(x, t)= \\
-J x-\frac{c \phi_{0}}{16 \pi^{3} \lambda^{2}} \int_{-d / 2}^{d / 2} \ln \left|\frac{\cos \frac{\pi}{2 d}(x+u)}{\sin \frac{\pi}{2 d}(x-u)}\right| \theta^{\prime}(u, t) d u .
\end{gathered}
$$

Using (117) we calculate $J_{y}(x, 0)=-\partial_{x} g(x, 0)$ and integrate the result by parts:

$$
\begin{gathered}
J_{y}(x, 0)=J- \\
\frac{c \phi_{0}}{32 \pi^{2} \lambda^{2} d} \int_{-d / 2}^{d / 2}\left[\cot \frac{\pi}{2 d}(x-u)+\tan \frac{\pi}{2 d}(x+u)\right] \theta^{\prime}(u) d u \\
=J+\frac{c \phi_{0}}{16 \pi^{3} \lambda^{2}}\left[\ln \left|\frac{\sin \pi x / d-\sin \pi u / d}{2}\right| \theta^{\prime}(u)\right]_{-d / 2}^{d / 2}+ \\
\frac{c \phi_{0}}{16 \pi^{3} \lambda^{2}} \int_{-d / 2}^{d / 2} \ln \left|\frac{2}{\sin \pi x / d-\sin \pi u / d}\right| \theta^{\prime \prime}(u, t) d u
\end{gathered}
$$

Here $\theta^{\prime}( \pm d / 2)=0$ because $J_{x}( \pm d / 2)=0$ at the ends of the junction. Equating $J_{y}$ to the sum of Josephson, resistive, and displacement current densities, we obtain:

$$
\begin{gathered}
\ddot{\theta}+\eta \dot{\theta}+\sin \theta-\beta= \\
\left(\frac{\lambda_{J}^{2}}{\pi \lambda}\right) \int_{-d / 2}^{d / 2} \ln \left|\frac{2}{\sin \pi x / d-\sin \pi u / d}\right| \theta^{\prime \prime}(u) d u
\end{gathered}
$$

where $\beta=J / J_{c}$. Equation (119) in which $x$ and $u$ are expressed in units of $d$, and $\epsilon=\lambda_{J}^{2} / \pi \lambda d$ reduces to (106) which was used in our simulations.

Now we turn to $\theta(x, t)$ after the transition from the vortex to a phase slip state in which

$$
\theta(x, t)=\theta_{0}(t)+\theta_{s}(x)
$$

where $\theta_{0}(t)$ satisfies the equation for a point JJ:

$$
\ddot{\theta}_{0}+\eta \dot{\theta}_{0}+\sin \theta_{0}=\beta_{0} .
$$


The small stationary term $\theta_{s}(x)$ results from the slight inhomogeneity of $\beta(x)=(1-k x) \beta_{0}$ due to weak screening. Substituting (120) into (119) we see that the term $\sin \theta \simeq \sin \theta_{0}(t)+$ $\theta_{s} \cos \theta_{0}(t)$ oscillates rapidly so $\theta_{s}(x) \cos \theta_{0}(t)$ yields a small dynamic correction $\delta \theta(x, t)$ to $\theta(x, t)$ which is negligible at large $\beta$ and small $k$ we are interested in. The static $\theta_{s}$ is then determined by (119) written in the dimensionless form:

$$
\epsilon \int_{-1 / 2}^{1 / 2} \ln \left|\frac{2}{\sin \pi x-\sin \pi u}\right| \theta_{s}^{\prime \prime}(u) d u=k x \beta_{0} .
$$

Instead of solving (122), it is more convenient to use (103) with $H=0$ and write $\theta_{s}(x)$ in the form which satisfies the boundary conditions $\theta_{s}^{\prime}( \pm 1 / 2)=0$ :

$$
\theta_{s}(x)=\sum_{n=0}^{\infty} \theta_{n} \sin q_{n} x
$$

where $q_{n}=\pi(2 n+1) / d$. Solution of $(103)$ is then

$$
g(x, y)=-\frac{c \phi_{0}}{16 \pi^{2} \lambda^{2}} \sum_{n=0}^{\infty} \theta_{n} e^{-q_{n}|y|} \cos q_{n} x .
$$

Next we use the condition $k J x / d=-\partial_{x} g(x, 0)$, that is

$$
J k \frac{x}{d}=-\frac{c \phi_{0}}{16 \pi^{2} \lambda^{2}} \sum_{n=0}^{\infty} \theta_{n} q_{n} \sin q_{n} x .
$$

Multiplying both sides of (125) by $\sin q_{n^{\prime}} x$ and integrating from $-d / 2$ to $d / 2$ yields:

$$
\frac{2 J k(-1)^{n}}{d q_{n}^{2}}=-\frac{c \phi_{0} d q_{n} \theta_{n}}{32 \pi^{2} \lambda^{2}} .
$$

Hence, $\theta_{n}=-4 \beta_{0} k(-1)^{n} / \pi^{4} \epsilon(2 n+1)^{3}$, and

$$
\theta_{s}(x)=-\frac{4 \beta_{0} k}{\pi^{4} \epsilon} \sum_{n=0}^{\infty} \frac{(-1)^{n}}{(2 n+1)^{3}} \sin \frac{\pi x}{d}(2 n+1),
$$

where $\epsilon=\lambda_{J}^{2} / \pi \lambda d$ and $\beta_{0}=J / J_{c}$. 


\section{APPENDIX B}

\section{NUMERICAL METHODS}

I developed efficient numerical codes to solve the main partial differential and integrodifferential equations addressed above. Besides the incredible agreement of results with previous works and possible exact solutions, these equations were solved using different numerical methods to ensure the validity and equality of the obtained results. The spatial terms in above differential equations were treated using both method of lines (MOL) as a finite difference method and fast Fourier transform (FFT) as a spectral method. In both cases the differential equation is reduced to a system of coupled nonlinear ordinary differential equations that can be stepped forward in time with any of the standard time-stepping techniques. Here, I will explain briefly these two methods applied exclusively to main equations discussed above.

- Method of Lines: In this technique the spatial term is discretized using finite difference method over a uniform spatial computational box[148]. For instance, the second order spatial derivative $\theta_{x x}$ in (44) can be approximated as

$$
\theta^{\prime \prime} \approx \frac{\theta_{i+1}-2 \theta_{i}+\theta_{i-1}}{2 \Delta x^{2}}
$$

using commonly used second order, central approximation given from the Taylor series over the uniformly distributed spatial grid points of $x_{i} \in(0, L)$ where $i$ and $\Delta x$ denote position and spacing along the grid, respectively. Substitution of this term in (44) gives a system of approximating nonlinear ordinary differential equations,

$$
\ddot{\theta}_{i}+\eta \dot{\theta}_{i}=\frac{\theta_{i+1}-2 \theta_{i}+\theta_{i-1}}{2 \Delta x^{2}}-\sin \theta_{i}+\beta
$$

which can be integrated subject to given initial and boundary conditions using suitable ODE integrators (Runge-Kutta, Adams-Bashforth, ...).

- Fast Fourier Transform (FFT): This is the best known example of a spectral method used to solve differential equations. Fourier transform is essentially an eigenfunction expansion over all continuous wavenumbers $k$ which on a finite domain $x \in(-L / 2, L / 2)$ with $N=2^{n}$ points are given by $k \in(2 \pi / L)[-N / 2,-N / 2-$ 
$1, \ldots, 0,1, \ldots, N / 2-1]$. The algorithm, for instance applied to nonlocal equation in (75) begins by implementing a Fourier transform in $x$ which results in the system of differential equations

$$
\left(\frac{d^{2}}{d t^{2}}+\eta \frac{d}{d t}\right) \mathcal{F}(\theta)=-\lambda_{J}^{2} k^{2} \mathcal{F}(G) \mathcal{F}(\theta)-\mathcal{F}(\sin \theta)+\beta \delta(k) .
$$

that can be stepped forward in time using any standard technique. Here the operator "F $\mathcal{F}$ " denotes the spatial Fourier transform.

In both cases of the above the system of ODEs were solved by the multistep, variable order Adams-Bashforth-Moulton method [149] which is a predictor-corrector integrator used for problems with stringent error tolerances. The absolute and relative error tolerances were kept below $10^{-6}$. The codes were run long enough to ensure that a steady state solution is obtained. The programs were stable enough to be run at $\Delta x \sim 0.1$, although much finer spacings were used for accuracy purposes.

The singularity of the integral kernel $G(x)$ was handled in two different ways. If using finite difference method, the divergence in $x=0$ is avoided by shifting the singularity position from $x=0$ to $x=e p s$ where eps is the command for generating a machine precision number of the order of $\mathcal{O}\left(10^{-15}\right)$. The error introduced here in calculations is perfectly negligible compared to other error mechanisms. In FFT method the singularity is treated exactly since Fourier transform of $G(x)$ in most cases is a smooth function in $k$, i.e. $\mathcal{F}\left(K_{0}(x)\right)=\frac{\pi}{\sqrt{1+k^{2}}}$.

The infinite integral in nonlocal equation in (75) was transformed over a finite domain with length $L_{b}$. The length $L_{b}$ of computational box $x_{1}<x<x_{1}+L_{b}$ along the $x$-axis (either co-moving with the vortex or expanding with the phase pile) was taken large enough to assure no artifacts coming from possible reflected waves at $x=x_{1}$ and $x=x_{1}+L_{b}$. We set $\theta\left(x_{1}, t\right)-\sin ^{-1} \beta<10^{-6}$ and $\theta\left(x_{1}+L_{b}, t\right)-2 \pi-\sin ^{-1} \beta<10^{-6}$ and made sure that changing $L_{b}$ does not affect the results, where $L_{b}$ was typically taken at least three times larger than the spatial extent of $\theta(x, t)$, be it a single vortex or expanding phase pile. The steady state phase distribution $\theta(x-v t)$ in a uniformly moving vortex at a given $\beta$ was computed by solving the full dynamic equation (75) using the single-vortex solution calculated at a smaller preceding value of $\beta$ as an initial condition. The code then runs until the velocity of the vortex stabilizes to the accuracy better than $0.1 \%$.

The integral kernel in (106) is not of convolution form, therefore this equation was solved using finite difference method. The steady state phase distribution $\theta(x-v t)$ at a given $\beta$ was computed by solving the full dynamic equation in (106) using the solution obtained at slightly lower current $\beta-\delta \beta$ as initial conditions. The code then runs until a periodic solution 
- if applicable - is attained. The authenticity of the results was examined successfully by solving this equation using a slower, more simpler iterative method. 
- Sine-Gordon (MOL). A typical code written in MATHEMATICA used to solve the full dynamical equation in (44) in ac magnetic field using method of line technique to produce Fig. 28.

$\backslash[$ Beta $]=10 ; \backslash$ Alpha $]=0.01 ; \mathrm{L}=20 ; \mathrm{T}=20 * \mathbf{P i} ;$ hac $=4$; $\mathrm{sol}=\mathbf{N D S o l v e}[\{\backslash[\mathbf{B e t a}] * \mathbf{D}[\mathrm{u}[\mathrm{t}, \mathrm{x}], \quad\{\mathrm{t}\},\{\mathrm{t}\}]+\mathbf{D}[\mathrm{u}[\mathrm{t}, \mathrm{x}], \quad\{\mathrm{t}\}]$

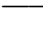
$\mathrm{D}[\mathrm{u}[\mathrm{t}, \mathrm{x}],\{\mathrm{x}\},\{\mathrm{x}\}]-\operatorname{Sin}[\mathrm{u}[\mathrm{t}, \mathrm{x}]], \mathrm{u}[0, \mathrm{x}]=0$, Derivative $[1,0][\mathrm{u}][0, \mathrm{x}]=0, \operatorname{Derivative}[0,1][\mathrm{u}][\mathrm{t}, 0]=$ hac $* \operatorname{Sin}[\backslash[$ Alpha $] * \mathrm{t}] * \operatorname{Tanh}[\mathrm{t}], \operatorname{Derivative}[0,1][\mathrm{u}][\mathrm{t}, \mathrm{L}]=0\}$, $\mathrm{u},\{\mathrm{t}, \mathrm{T}-2 * \mathbf{P i}, \mathrm{T}\},\{\mathrm{x}, 0, \mathrm{~L}\}$, MaxStepSize $\rightarrow 0.01$, MaxSteps $\left.\rightarrow 10^{\wedge} 8\right]$;

Plot3D [Evaluate [First $[\mathrm{D}[\mathrm{u}[\mathrm{t}, \mathrm{x}],\{\mathrm{x}\}] /$ sol $] /$ hac $]$, $\{\mathrm{t}, \mathrm{T}-2 * \mathbf{P i}, \mathrm{T}\},\{\mathrm{x}, 0, \mathrm{~L}\}$, PlotPoints $\rightarrow 300$, PlotRange $\rightarrow\{\{\mathrm{T}-(2 * \mathbf{P i}+1 / 2), \mathrm{T}\},\{0, \mathrm{~L}\},\{-1.2,6\}\}$, Ticks $\rightarrow$ Automatic, Automatic, $\{-1,0,1\}\}$, Mesh $\rightarrow$ None, ColorFunction $\rightarrow$ Hue, ViewPoint $\rightarrow\{-0.75,-2.25,0.75\}$, AspectRatio $\rightarrow 1$, AxesStyle $\rightarrow$ Directive [Thickness [0.006], 12],

AxesLabel $\rightarrow\{$ Style $[" \backslash[$ Omega $]$ t",

14, Bold, FontFamily $\rightarrow$ "Courier"], Style $[" \mathrm{x} / \backslash ! \backslash(\backslash *$ SubscriptBox $[\backslash(\backslash[$ Lambda $] \backslash), \sqcup \backslash(\mathrm{J} \backslash)] \backslash)$ ", 14, Bold, FontFamily $\rightarrow$ "Courier"], Rotate [ Style $[" \mathrm{~B}(\mathrm{x}, \mathrm{t}) / \backslash ! \backslash(\backslash *$ SubscriptBox $[\backslash(\mathrm{H} \backslash), \sqcup \backslash(\mathrm{ac} \backslash)] \backslash) ", \quad 14$, Bold, FontFamily $\rightarrow$ "Courier"], 90*Degree]\}, Boxed $\rightarrow$ False ] 
- Nonlocal sine-Gordon (MOL). Here we present a sample code written in MATLAB to solve the nonlocal sine-Gordon equation for bulk junction in (86) with the ratio of $\lambda_{J} / \lambda=10$ using method of lines technique.

\% this is the main script of code

clc

clear all

eta $=0.01 ; \quad$ \%low dissipation

lratio $=10 ; \quad$ Oratio is \lambda_J $\backslash l a m b d a_{-} L$

beta $=0.2$;

epsilon $=1.0 \mathrm{e}-12 ; \quad \%$ shift of singularity

$\%$ time domains

$\mathrm{t} 0=0 ; \mathrm{tf}=100$;

$\%$ spatial domains

$\mathrm{xl}=-80 ; \quad \mathrm{xu}=50 ; \quad \mathrm{dx}=0.01 ;$

$\mathrm{x}=\mathrm{xl}: \mathrm{dx}: \mathrm{xu} ; \quad \mathrm{n}=\operatorname{length}(\mathrm{x})$;

$\mathrm{dxq}=\mathrm{dx} / 20$

$\mathrm{xq}=\mathrm{xl}-1: \mathrm{dxq}: \mathrm{xu}+1 ;$

$\mathrm{d}=\operatorname{round}(1 / \mathrm{dxq})$;

$\%$ integral kernel

$\mathrm{m}=\operatorname{round}(\mathrm{n} / 2)$;

$\mathrm{xqq}=\mathrm{xq}((20 * \mathrm{~m}-19): 2 * \mathrm{~d}+20 * \mathrm{~m}-19)$;

$\mathrm{K}=\operatorname{besselk}\left(0\right.$, rratio $\left.* \operatorname{sqrt}\left((\mathrm{x}(\mathrm{m})-\mathrm{xqq}) \cdot{ }^{\wedge} 2+\operatorname{epsilon}{ }^{\wedge} 2\right)\right)$;

$\%$ initial conditions

$\mathrm{u} 0(1: \mathrm{n})=\operatorname{asin}($ gamma $)+4 * \operatorname{atan}(\exp (\mathrm{x}(1: \mathrm{n}))) ;$

$\mathrm{u} 0(\mathrm{n}+1: 2 * \mathrm{n})=0$;

$\%$ Ode integration

$\%$ options 


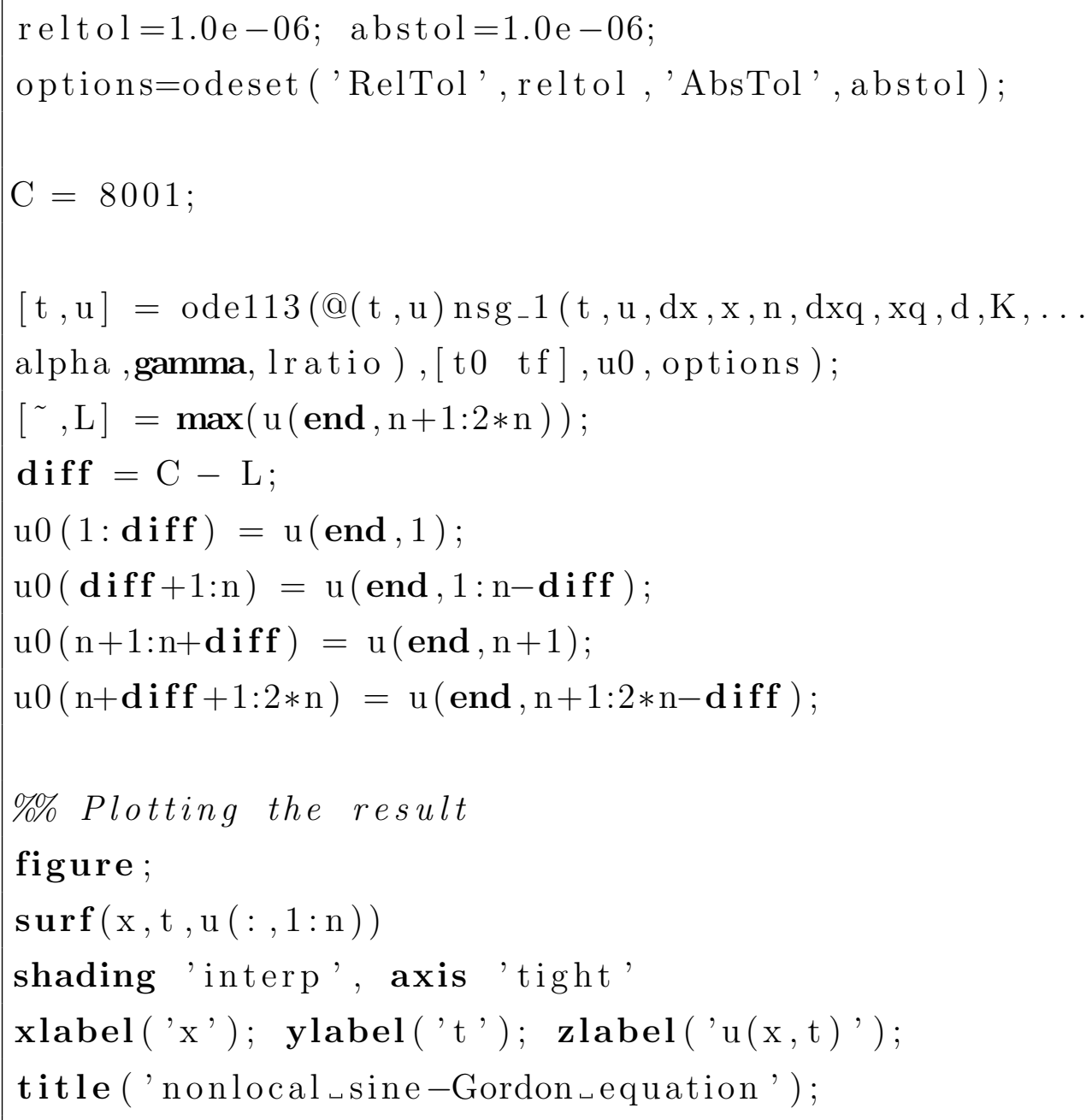

function $u t=n_{s} g_{-1}(\sim, u, d x, x, n, d x q, x q, d, K$, alpha, gamma, lratio $)$

\% Function nsg-1 computes the $t$ derivative vector

\%for the nonlocal sine-Gordon equation

$\%$ B.C.

$\mathrm{u} 1=\operatorname{smooth}(\mathrm{u}(1: \mathrm{n})$,' lowess');

$\mathrm{u} 2=\operatorname{smooth}(\mathrm{u}(\mathrm{n}+1: 2 * \mathrm{n})$, 'lowess' $)$;

$\mathrm{uxx}=4 * \operatorname{del} 2(\mathrm{u} 1, \mathrm{dx})$;

$\operatorname{uxx}(1)=0 ; \operatorname{uxx}(n)=0 ;$

uxxin $=$ interp1 (x, uxx, xq, 'spline' );

$\mathrm{N}=\operatorname{length}(\mathrm{uxxin})$; 


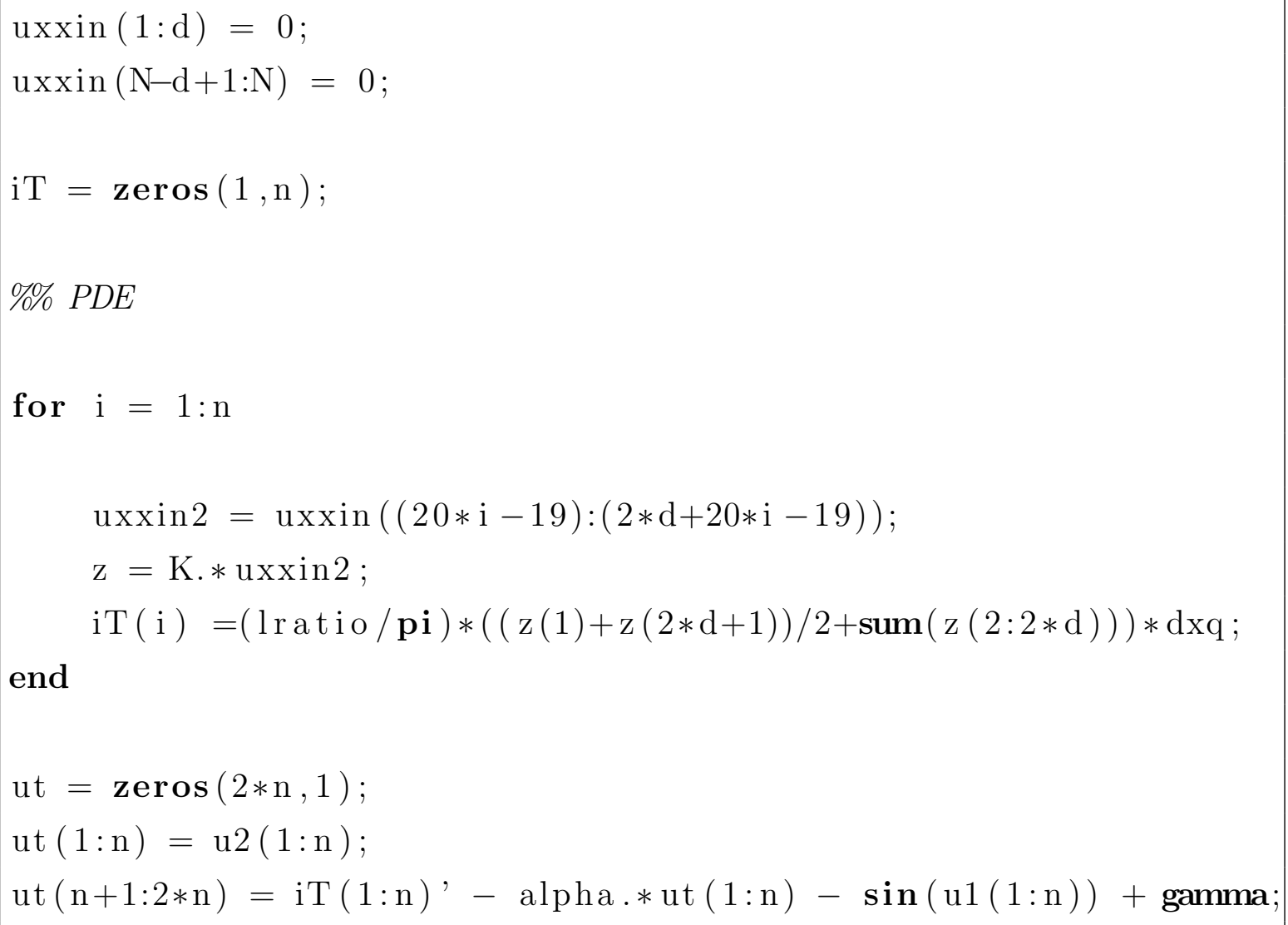


- Nonlocal sine-Gordon (FFT). Here is a sample code written in MATLAB to solve the same nonlocal dynamic equation in (86) using the FFT method.

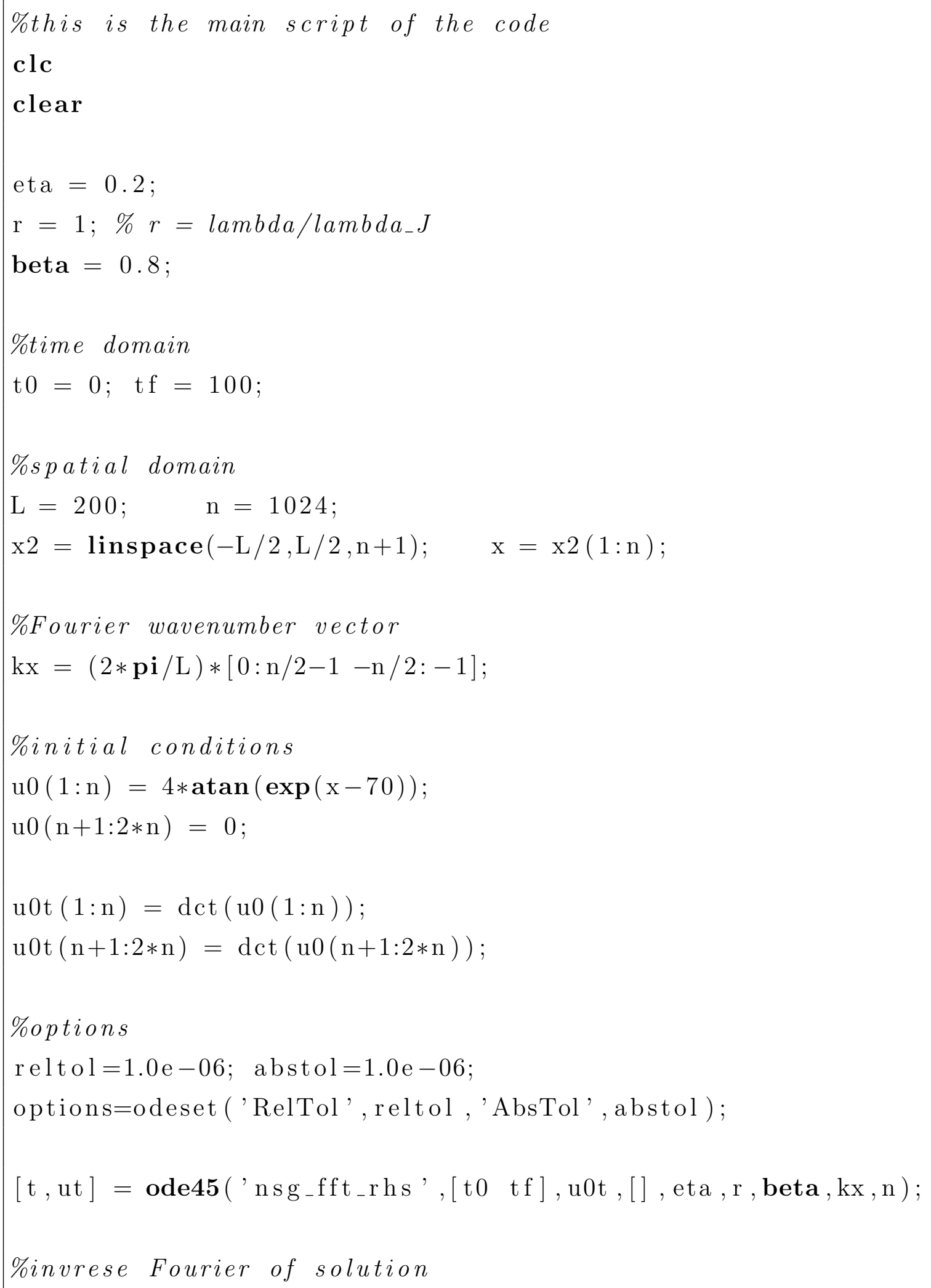




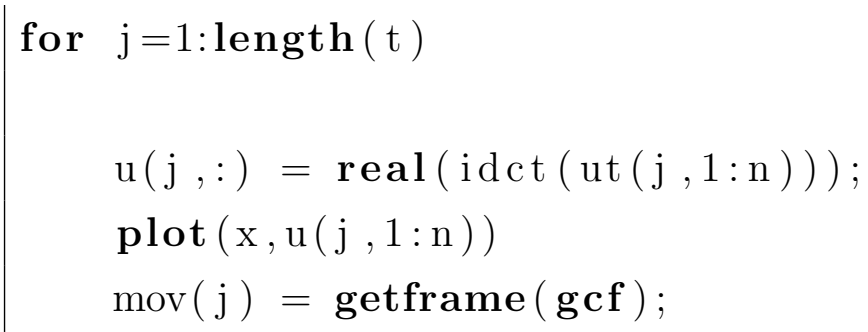

end

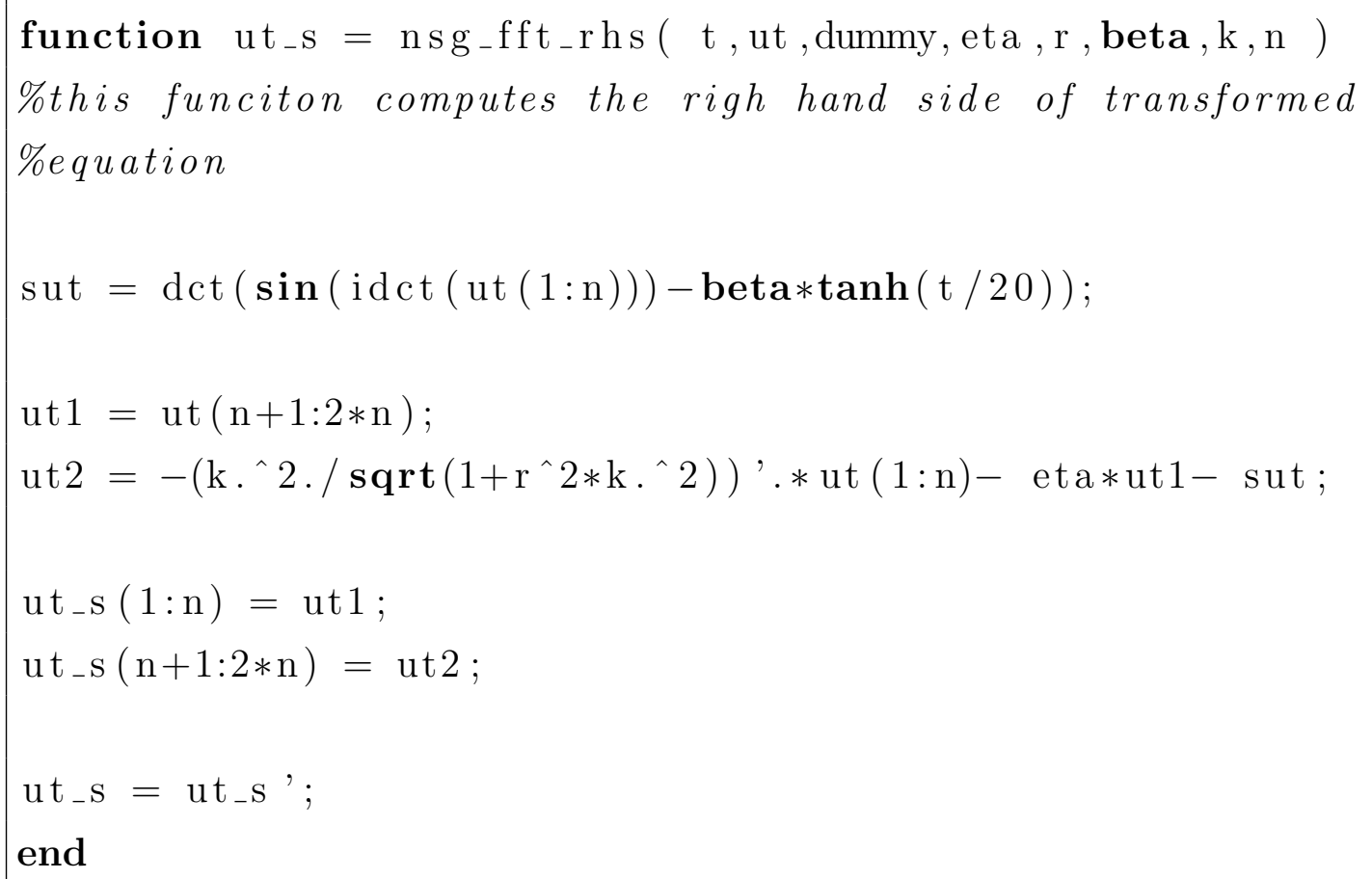




\section{VITA}

Ahmad K. Sheikhzada

Department of Physics

Old Dominion University

Norfolk, VA 23529

\section{Publications:}

- A. Sheikhzada and A. Gurevich, "Nonlinear dynamics of Josephson vortices in a film screen under dc and ac magnetic fields", Physica C 506, 59-68 (2014).

- A. Sheikhzada and A. Gurevich, "Fragmentation of fast Josephson vortices and breakdown of ordered states by moving topological defects", Scientific Reports 5, 17821 (2015).

- A. Sheikhzada and A. Gurevich, "Dynamic transition of vortices into phase slips and generation of vortex-antivortex pairs in thin film Josephson junctions under dc and ac currents.", submitted to PRB, arXiv.org/abs/1703.02843, (2017).

\section{Presentations:}

- A. Sheikhzada and A. Gurevich, "New instability of driven Josephson vortices in long underdamped junctions", APS March Meeting, Baltimore MD, 2016.

- A. Sheikhzada and A. Gurevich, "RF dissipation due to nonlinear dynamics of Josephson vortices in polycrystalline thin film SRF structures", 7th International Workshop on Thin Films and New Ideas for Pushing the Limits of RF Superconductivity, Jefferson Lab, Newport News VA, 2016.

- A. Sheikhzada and A. Gurevich, "Dynamic transitions of vortices into phase slips in Josephson junctions under dc and ac currents", APS March Meeting, New Orleans LA, 2017.

Typeset using $\mathrm{AT}_{\mathrm{EX}}$. 